\title{
Anregbarkeit der Kelvin-Helmholtz-Instabilität in der freien Scherströmung hinter einer scharfen Kante
}

\author{
Dissertation \\ zur Erlangung des Doktorgrades \\ der Mathematisch-Naturwissenschaftlichen Fakultäten \\ der Georg-August-Universität zu Göttingen
}

vorgelegt von

Hironori Tokuno

geboren in

Sakata-Yamagata/Japan

Göttingen 2004 
D7

Referent: Prof. Dr. Dirk Ronneberger

Korreferent: $\quad$ Prof. Dr. Werner Lauterborn

Tag der mündlichen Prüfung: 25. Oktober 2004 


\section{Kurzfassung}

Die Anregung und die Unterdrückung von Instabilitätswellen in einer abgelösten Scherschicht werden bei verschiedenen Strouhalzahlen experimentell untersucht, um den Anregungsmechanismus der Instabilitätswelle zu verstehen. Die Scherschicht löst dabei von der Hinterkante einer ebenen, einseitig überströmten Platte ab. Die Instationarität wird einerseits durch ferne Quellen (schwingende Membranen in der Kanalwand), andererseits durch lokale Schwingungen der Hinterkante, die in der vorliegenden Arbeit neu gebaut wurde, erzeugt.

Die genaue Beschreibung der Wechselwirkung zwischen einer Gleichströmung und einer durch die Instationaritäten erzeugten Wechselströmung ist sehr komplex, da die Dynamik nicht nur durch die Instabilitätswelle, sondern auch durch instationäre Ablösebedingungen im Kantenbereich dominiert wird. Um diese Dynamik zu untersuchen, wird ein Versuch unternommen, das gemessene zur Anregung kohärente Wechselfeld in drei Teile zu zerlegen: das Feld der Instabilitätswelle, das anregende Feld bei ruhendem Medium und das Nahfeld, das nicht zu den ersten zwei Feldern gehört. Bei der Beschreibung des Feldes der Instabilitätswelle wird ihre von der Inhomogenität der Gleichströmung verursachte räumliche Änderung in Strömungsrichtung durch Anpassung an Messwerte berücksichtigt und mit einem theoretischen Ansatz (WKBMethode) verglichen. Dabei wird eine Wechselwirkung von Moden der Instabilitätswelle beobachtet: die angefachte Mode wird in die gedämpfte Mode gestreut. Das anregende Feld beim ruhenden Medium wird mit einer numerischen Simulation bestimmt. Das Nahfeld kann anschließend durch die Subtraktion der ersten beiden Felder von dem Gesamtfeld bestimmt werden. Bei dem Betrag der $\tilde{u}$-Komponete des Nahfeldes zeigt sich, dass ein Teil des Nahfeldes durch Verschiebungen der Scherschicht, also durch scheinbare Wechselgeschwindigkeiten, zustande gekommen ist.

Zur genauen Untersuchung des Nahfeldes werden Kompensationsexperimente durchgeführt, in denen zwei unterschiedliche Anregungsvorrichtungen gleichzeitig so betrieben werden, dass die Amplitude der Instabilitätswelle im Fernfeld verschwindet. Durch das Kompensationsexperiment kann ein nichtanregendes Nahfeld hergestellt und somit ermittelt werden, in dem keine Quellen der Instabilitätswelle mehr vorhanden sind oder sich alle Quellen gegenseitig kompensieren. Beim Vergleich des nichtanregenden mit dem anregenden Nahfeld zeigt sich, dass das nichtanregende Nahfeld vollständiger durch das scheinbare Geschwindigkeitsfeld beschrieben werden kann als das anregende Nahfeld. Dieses Ergebnis ist einerseits sinnvoll, da im homogenen Fall scheinbare Verschiebungen der Gleichströmung nicht zur Anregung der Instabilitätswelle führen, und im inhomogenen Fall auch anzunehmen ist, dass sich Instabilitätswellen nicht durch ein Nahfeld anregen lassen, das sich als ein scheinbares Geschwindigkeitsfeld beschreiben lässt. Andererseits würde es bedeuten, dass durch diese scheinbare Verschiebung verursachte Druckfelder für die Anregung der Instabilitätswelle verantwortlich sind, da aufgrund der Inhomogenität des anregenden Feld das scheinbare Geschwindigkeitsfeld nicht divergenzfrei ist. 



\section{Inhaltsverzeichnis}

1 Einleitung 1

2 Der experimentelle Aufbau und das Messverfahren $\quad 5$

2.1 Der Wasserkanal . . . . . . . . . . . . . . . . . . . 5

2.2 Die Anregungsvorrichtungen . . . . . . . . . . . . . . . 8

2.2.1 Die Druckgradienten-Anregung in den Seitenwänden des Kanals 8

2.2.2 Die lokale Anregung durch eine Schwinghinterkante . . . . . . . 9

2.3 Die Messdatenerfassung . . . . . . . . . . . . . . . . . . . . 12

2.3.1 LDA-Messung . . . . . . . . . . . . . . . . . . . 12

2.3.2 Anpassung der Messwerte mittels phasenstarrer Mittelung . . . 15

2.3.3 Ablauf der Messung . . . . . . . . . . . . . . . . 16

3 Die Gleichströmung $\quad 19$

3.1 Die $\bar{u}$-Komponente . . . . . . . . . . . . . . . . . . . . . . . . . . . . . . . . . . 19

3.1.1 Das Grenzschicht- und das Scherschichtprofil . . . . . . . . . . . 20

3.1 .2 Strömungsparameter . . . . . . . . . . . . . . . . 23

3.1.3 Inhomogenität des $\bar{u}$-Profils . . . . . . . . . . . . . . . . . . . 24

3.2 Die $\bar{v}$-Komponente . . . . . . . . . . . . . . . . . . . . . . . . . . . . . 24

3.3 Die Gleichwirbeligkeit . . . . . . . . . . . . . . 26

4 Anregbarkeit der Kelvin-Helmholtz-Instabilität 29

4.1 Die Abhängigkeit der Instabilitätswelle von der Strouhalzahl . . . . . . 29

4.1.1 Die Dispersionsbeziehung . . . . . . . . . . . . . . . . . . 30

4.1.2 Die Abhängigkeit der $\tilde{v}$-Komponente von der Strouhalzahl . . . 31

4.1.3 Vergleich der Dispersionsbeziehung mit Messwerten . . . . . . . 35

4.2 Untersuchung des instationären Strömungsfeldes . . . . . . . . . . . . . . . 37

4.2.1 Zerlegung des Messfeldes . . . . . . . . . . . . . . . . 38

4.2.2 Das Profil der Kelvin-Helmholtz-Instabilitätswellen .... . . 50

4.2.3 Berücksichtigung der $x$-Abhängigkeit des Gleichströmungsfeldes $\quad 57$

4.2.4 Überblick über das Nahfeld . . . . . . . . . . . . . . . . . 67

5 Untersuchung der Nahfelder mittels Kompensationsexperiment $\quad 71$

5.1 Das Kompensationsexperiment . . . . . . . . . . . . . . . 71

5.1 .1 Die Qualität der Kompensation . . . . . . . . . . . . . 72

5.1.2 Die Kompensation in Abhängigkeit von der Anregungsfrequenz 75 
5.2 Das nichtanregende Nahfeld . . . . . . . . . . . . . . . . . . . . . . . . 78

5.2.1 Die angefachte und gedämpfte Mode der KH-Wellen im Kompensationsfeld . . . . . . . . . . . . . . . . . 80

5.2.2 Die lineare Beschreibung des Nahfeldes . . . . . . . . . . . . . . 80

5.2.3 Vergleich des nichtanregenden mit dem anregenden Nahfeld . . 88

6 Schlussfolgerung und Ausblick $\quad 93$

6.1 Schlussfolgerung . . . . . . . . . . . . . . . . . . . . . . . . . . . 93

6.2 Ausblick . . . . . . . . . . . . . . . . . . . . . 95

$\begin{array}{ll}\text { A Das anregende Strömungsfeld } & 97\end{array}$

A.1 Das numerische Verfahren . . . . . . . . . . . . . . . . . . . 97

A.1.1 Die CIP-Methode . . . . . . . . . . . . . . . . . . . . 97

A.1.2 Das verwendete räumliche Gitter . . . . . . . . . . . . . . 99

A.1.3 Randbedingungen . . . . . . . . . . . . . . . . . . . 101

A.1.4 Das Flussdiagramm der numerischen Rechnung . . . . . . . . . 101

A.2 Vergleich der numerischen Lösung mit Messwerten . . . . . . . . . . . . 102

A.2.1 Vergleich mit Messwerten an verschiedenen Stellen . . . . . . . 105

A.2.2 Vergleich mit Messwerten bei verschiedenen Frequenzen . . . . . 106

Literaturverzeichnis 


\section{Abbildungsverzeichnis}

2.1 Der bei der Messung verwendete Wasserkanal . . . . . . . . . . . . . 6

2.2 Schematischer Aufbau des Temperaturregelkreises im Wasserkanal . . . 8

2.3 Messstrecke des Wasserkanals mit der Druckgradienten-Anregung bzw. mit der Schwingkante . . . . . . . . . . . . . . . . . 9 9

2.4 Die verwendete Splitterplatte mit schwingender Hinterkante . . . . . . 10

2.5 Seitenansicht der Schwingkanten-Anregung . . . . . . . . . . . . . . . . 10

2.6 Die durch die schwingende Hinterkante angeregte Wechselgeschwindigkeit (u-Komponente) in Abhängigkeit von der $z$-Koordinate bei $1.6 \mathrm{~Hz}$, wobei $U_{1}=15 \mathrm{~cm} / \mathrm{s}, x=30 \mathrm{~mm}$ und $y=2 \mathrm{~mm}$ : (a) der Betrag und (b) die Phase . . . . . . . . . . . . . . . . . .

3.1 Die Gleichgeschwindigkeitskomponente $\bar{u}$ in der Plattengrenzschicht und der Übergang in eine Scherschicht stromab der Hinterkante der Splitterplatte . . . . . . . . . . . . . . . . . .

3.2 (a) Das Grenzschichtprofil bei $x=-30 \mathrm{~mm}$, mit angepasstem Blasiusprofil mit Pohlhausen-Korrektur und (b) Effektivwerte der turbulenten Fluktuationen $u_{\text {eff }}^{\prime}(y)$, die Unsicherheit der Messwerte $\Delta \bar{u}(y)$ und der Anpassungsfehler $F$ als Funktion von $y$. . . . . . . . . . . . . . .

3.3 (a) Das Scherschichtprofil bei $x=30 \mathrm{~mm}$ mit der angepassten Funktion $\bar{u}(y)$ sowie deren 1. und 2. Ableitung, (b) Effektivwerte der Fluktuationen $u_{\text {eff }}^{\prime}(y)$, die Unsicherheit der Messwerte $\Delta \bar{u}(y)$ und der Anpassungsfehler $F$ als Funktion von $y$. . . . . . . . . . . . . . . . . . . . 22

3.4 Die Änderung des $\bar{u}$-Profils in $x$-Richtung: $\partial \bar{u} / \partial x \ldots \ldots \ldots$

3.5 (a) $\bar{u}$ bei $y=0$ als Funktion von $x$ und (b) die Impulsverlustdicke $\Theta$ als Funktion von $x \ldots \ldots \ldots \ldots \ldots \ldots$

3.6 Die Gleichgeschwindigkeitskomponente $\bar{v}$ in der Plattengrenzschicht und der Übergang in eine Scherschicht stromab der Hinterkante der Splitter-

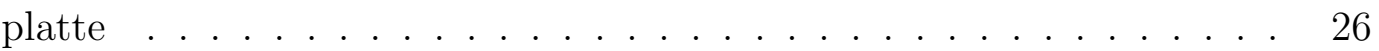

3.7 Die Wirbeldicke $\delta_{\omega}$ als Funktion von $x \ldots \ldots . \ldots . . \ldots 27$

3.8 Übersicht über die Gleichwirbeligkeit $\bar{\omega}$ : dreidimensionale Darstellung (oben) und Konturlinienbild (unten) . . . . . . . . . . .

4.1 Die Phasenkonstante $\operatorname{Re}\{\alpha\}$ und Anfachungskonstante $\operatorname{Im}\{\alpha\}$ der Instabilitätswelle in der Scherschicht bei $x=30 \mathrm{~mm}$, mit der Impulsverlustdicke $\Theta_{0}$ normiert . . . . . . . . . . . . . . . . . . . 
4.2 Übersicht über den Betrag der $\tilde{v}$-Komponente bei Anregung mit dem Druckgradienten als Funktion der $x$-Koordinate und der Anregungsfrequenz. (Oben: lineare Auftragung, unten: logarithmische Auftragung) .

4.3 Betrag und Phase der $\tilde{v}$-Komponente als Funktion der Strouhalzahl bei $y=0 \mathrm{~mm}$ für verschiedene $x$-Koordinaten: (a) Betrag und (b) Phase . .

4.4 Vergleich zwischen Theorie und Experiment: Normierte Phasenkonstante $\operatorname{Re}\{\alpha\}$ und Anfachungskonstante $\operatorname{Im}\{\alpha\}$ der Instabilitätswelle in der Scherschicht als Funktion von $S t_{\Theta} \ldots \ldots \ldots . \ldots . \ldots 36$

4.5 Übersicht über das Messpunktgitter im Bereich der Ablösekante . . . . .

4.6 Übersicht über den Betrag der normierten $\tilde{v}$-Komponente der Wechselgeschwindigkeit bei $0.8 \mathrm{~Hz}$ : (a) das Gesamtfeld $\tilde{v}_{A l l}^{D r}$; (b) $\tilde{v}_{K H+N F}^{D r}$ (das anregende Feld ist vom Gesamtfeld abgezogen) . . . . . . . . . . . .

4.7 Übersicht über den Betrag der normierten $\tilde{v}$-Komponente der Wechselgeschwindigkeit bei $1.6 \mathrm{~Hz}$ : (a) das Gesamtfeld $\tilde{v}_{A l l}^{D r}$; (b) $\tilde{v}_{K H+N F}^{D r}$ (das anregende Feld ist vom Gesamtfeld abgezogen) . . . . . . . . . . . .

4.8 Übersicht über den Betrag der normierten $\tilde{v}$-Komponente der Wechselgeschwindigkeit bei $2.4 \mathrm{~Hz}$ : (a) das Gesamtfeld $\tilde{v}_{A l l}^{D r}$; (b) $\tilde{v}_{K H+N F}^{D r}$ (das anregende Feld ist vom Gesamtfeld abgezogen) . . . . . . . . . . . .

4.9 Übersicht über den Betrag der normierten $\tilde{u}$-Komponente der Wechselgeschwindigkeit bei $0.8 \mathrm{~Hz}$ : (a) das Gesamtfeld $\tilde{u}_{A l l}^{D r}$; (b) $\tilde{u}_{K H+N F}^{D r}$ (das anregende Feld ist vom Gesamtfeld abgezogen) . . . . . . . . . . .

4.10 Übersicht über den Betrag der normierten $\tilde{u}$-Komponente der Wechselgeschwindigkeit bei $1.6 \mathrm{~Hz}$ : (a) das Gesamtfeld $\tilde{u}_{A l l}^{D r}$; (b) $\tilde{u}_{K H+N F}^{D r}$ (das anregende Feld ist vom Gesamtfeld abgezogen) . . . . . . . . . . . . .

4.11 Übersicht über den Betrag der normierten $\tilde{u}$-Komponente der Wechselgeschwindigkeit bei $2.4 \mathrm{~Hz}$ : (a) das Gesamtfeld $\tilde{u}_{A l l}^{D r}$; (b) $\tilde{u}_{K H+N F}^{D r}$ (das anregende Feld ist vom Gesamtfeld abgezogen) . . . . . . . . . . .

4.12 Übersicht über den Betrag der normierten Wechselgeschwindigkeit $\tilde{u}_{K H+N F-s}$ ohne die scheinbare Geschwindigkeit $\tilde{u}_{s}$ bei (a) $0.8 \mathrm{~Hz}$, (b)

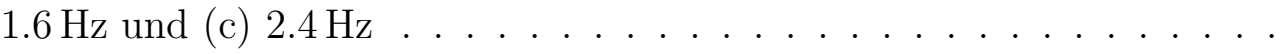

4.13 Vergleich zwischen gemessenen und theoretischen KH-Wellenprofilen $\tilde{u}_{K H}^{D r}, \tilde{v}_{K H}^{D r}$ in der Scherschicht bei $x=10 \mathrm{~mm}$ für $2.4 \mathrm{~Hz}$ : (a) Vergleich der Beträge zwischen $\tilde{u}_{K H+N F}^{D r}$ und $\tilde{u}_{K H}^{D r}$ sowie $\tilde{v}_{K H+N F}^{D r}$ und $\tilde{v}_{K H}^{D r}(\mathrm{~b})$ Vergleich zwischen $\left|\tilde{u}_{K H+N F-s}^{D r}\right|$ und $\left|\tilde{u}_{K H}^{D r}\right|$ sowie $\left|\tilde{v}_{K H+N F-s}^{D r}\right|$ und $\left|\tilde{v}_{K H}^{D r}\right|$ (c) Vergleich der Phasen von $\tilde{u}_{K H+N F}^{D r}$ und $\tilde{u}_{K H}^{D r}$ sowie von $\tilde{v}_{K H+N F}^{D r}$ und $\tilde{v}_{K H}^{D r}(\mathrm{~d})$ Vergleich der Phasen von $\tilde{u}_{K H+N F-s}^{D r}$ und $\tilde{u}_{K H}^{D r}$ sowie von $\tilde{v}_{K H+N F-s}^{D r}$ und $\tilde{v}_{K H}^{D r} \quad 52$

4.14 Vergleich der Messwerte $\left|\tilde{u}_{K H+N F-s}^{D r}\right|,\left|\tilde{v}_{K H+N F-s}^{D r}\right|$ mit den durch die Rayleigh-Gleichung bestimmten Theoriekurven $\left|\tilde{u}_{K H}^{D r}\right|,\left|\tilde{v}_{K H}^{D r}\right|$ in der Scherschicht bei $x=10 \mathrm{~mm}$ : (a) $f=0.8 \mathrm{~Hz}$, (b) $f=1.2 \mathrm{~Hz}$, (c) $f=1.6 \mathrm{~Hz}$ und $(\mathrm{d}) f=2.0 \mathrm{~Hz} \ldots \ldots \ldots \ldots \ldots$

4.15 Vergleich der Phasen von $\tilde{u}_{K H+N F-s}^{D r}$ und $\tilde{v}_{K H+N F-s}^{D r}$ mit den aus der Rayleigh-Gleichung resultierenden $\arg \left\{\tilde{u}_{K H}^{D r}\right\}$ und $\arg \left\{\tilde{v}_{K H}^{D r}\right\}$ in der Scherschicht bei $x=10 \mathrm{~mm}$ : (a) $f=0.8 \mathrm{~Hz}$, (b) $f=1.2 \mathrm{~Hz}$, (c) $f=1.6 \mathrm{~Hz}$ und $(\mathrm{d}) f=2.0 \mathrm{~Hz} \ldots \ldots \ldots \ldots$ 
4.16 Energietransfer zwischen Gleich- und Wechselströmung bei $x=$ $10 \mathrm{~mm}$ für verschiedene Frequenzen bei Druckgradienten-Anregung: Abhängigkeit von der $y$-Koordinate (a) und von der Anregungsfrequenz

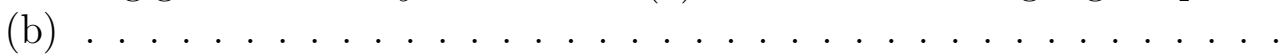

4.17 Die durch die Rayleigh-Gleichung bestimmte lokale Wellenzahl (mit der Impulsverlustdicke $\Theta_{0}$ normiert) in Anhängigkeit von der $x$-Koordinate bei einer Anregungsfrequenz von $1.6 \mathrm{~Hz}$. . . . . . . . . . . . . . . .

4.18 Vergleich der Messwerte $\left|\tilde{u}_{K H+N F-s}^{D r}\right|,\left|\tilde{v}_{K H+N F-s}^{D r}\right|$ mit den durch Ansatz (Gleichung (4.21)) bestimmten Theoriekurven $\left|\tilde{u}_{K H}^{D r}\right|,\left|\tilde{v}_{K H}^{D r}\right|$ in der Scherschicht bei verschiedenen $x$-Koordinaten für $1.6 \mathrm{~Hz}$ : (a) $x=0.4 \mathrm{~mm}$, (b) $x=3 \mathrm{~mm},(\mathrm{c}) x=6 \mathrm{~mm}$ und (d) $x=10 \mathrm{~mm} \ldots \ldots \ldots$

4.19 Vergleich der gemessenen Phasen von $\tilde{u}_{K H+N F-s}^{D r}$ und $\tilde{v}_{K H+N F-s}^{D r}$ mit den durch Ansatz (Gleichung (4.21)) bestimmten Theoriekurven $\tilde{u}_{K H}^{D r}, \tilde{v}_{K H}^{D r}$ in der Scherschicht bei verschiedenen $x$-Koordinaten für $1.6 \mathrm{~Hz}$ : (a) $x=$ $0.4 \mathrm{~mm},(\mathrm{~b}) x=3 \mathrm{~mm}$, (c) $x=6 \mathrm{~mm}$ und (d) $x=10 \mathrm{~mm} \ldots . .$.

4.20 Die Abhängigkeit der durch Anpassung bestimmten Vorfaktoren $C_{A}$ und $C_{D}$ von der $x$-Koordinate (Oben: Betrag, unten: Phase). . . . . . . . .

4.21 Vergleich der relativen Änderung des $\bar{u}$-Profils in $x$-Richtung $k$ mit der Wellenzahl $|\alpha|$ bei verschiedenen Frequenzen . . . . . . . . . .

4.22 Vergleich der Vorfaktoren $C_{A}(x)$ (Anpassung an Messwerte) mit $A(x)$ (WKB-Methode) für die angefachte Lösung der KH-Welle bei fünf verschiedenen Frequenzen . . . . . . . . . . . . . .

4.23 Der durch Anpassung an Messwerte bestimmte Vorfaktor $C_{D}(x)$ für die gedämpfte Lösung der KH-Welle . . . . . . . . . . . . . . . . .

4.24 Übersicht über den Betrag des durch die Druckgradienten-Anregung erzeugten Nahfeldes bei $1.6 \mathrm{~Hz}$ : (a) die $\tilde{u}$ - und (b) die $\tilde{v}$-Komponente . . .

5.1 Wechselgeschwindigkeiten der Kompensationsfelder und der durch die Schwingkanten-Anregung sowie durch Druckgradienten-Anregung erzeugten Felder bei $x=30 \mathrm{~mm}$ für $1.6 \mathrm{~Hz}$ : Betrag (a) und Phase (b) der $\tilde{u}$-Komponente, Betrag (c) und Phase (d) der $\tilde{v}$-Komponente . . . .

5.2 Übersicht über den Betrag der Wechselgeschwindigkeit im Kompensationsfeld bei $1.6 \mathrm{~Hz}$ : oben die $\tilde{u}$ - und unten die $\tilde{v}$-Komponente . . . . . .

5.3 Betrag der Wechselgeschwindigkeiten des Kompensationsfeldes und des durch Schwingkanten-Anregung erzeugten Feldes bei $x=60 \mathrm{~mm}$ für $1.6 \mathrm{~Hz}$ : oben die $\tilde{u}$ - und unten die $\tilde{v}$-Komponente . . . . . . . . . . . .

5.4 (a) Der Kompensationsparameter $K$ in Abhängigkeit von der $x$ Koordinate für die verschiedenen Anregungusfrequenzen in der komplexen Ebene, (b) Abhängigkeit der Kompensationsparameter $|\bar{K}|$ und $\Delta \bar{\varphi}_{K}$ sowie $\Delta T_{K}$ von der Anregungsfrequenz . . . . . . . . .

5.5 Kompensationsfeld ohne das anregende Feld $\tilde{\psi}_{K H_{D}+N F}^{K o m}$ im Nahbereich der Hinterkante bei $1.6 \mathrm{~Hz}$ : der mit $V_{k}$ normierte Betrag der $\tilde{u}$-Komponente (a) und der $\tilde{v}$-Komponente (b) . . . . . . . . . . . . . . . . 
5.6 Die durch den Ansatz (Gleichung (4.21)) bestimmten Vorfaktoren $C_{A}$ und $C_{D}$ im Kompensationsfeld bei $1.6 \mathrm{~Hz}$ : (a) der Betrag von $C_{A}$, (b) die Phase von $C_{A}$, (c) der Betrag von $C_{D}$ und (d) die Phase von $C_{D}$. .

5.7 Vergleich des Nahfeldes im Kompensationsexperiment $\tilde{\psi}_{N F}^{K o m}$ (a) mit dem durch Überlagerung von $\tilde{\psi}_{N F}^{K l}$ und $\tilde{\psi}_{N F}^{D r}$ bestimmten Nahfeld $\tilde{\psi}_{N F}^{K l D r}(\mathrm{~b})$ bei $1.6 \mathrm{~Hz}$ : der mit $V_{k}$ normierte Betrag der $\tilde{u}$-Komponente . . . . . . . . .

5.8 Vergleich des Nahfeldes im Kompensationsexperiment $\tilde{\psi}_{N F}^{K o m}$ (a) mit dem durch die Überlagerung von $\tilde{\psi}_{N F}^{K l}$ und $\tilde{\psi}_{N F}^{D r}$ bestimmten Nahfeld $\tilde{\psi}_{N F}^{K l D r}(\mathrm{~b})$ bei $1.6 \mathrm{~Hz}$ : der mit $V_{k}$ normierte Betrag der $\tilde{v}$-Komponente . . . . . . .

5.9 Vergleich der Nahfelder im Kompensationsexperiment $\tilde{\psi}_{N F}^{K o m}$ (blaue Kurve) und bei Überlagerung von $\tilde{\psi}_{N F}^{K l}+\tilde{\psi}_{N F}^{D r}=\tilde{\psi}_{N F}^{K l D r}$ (rote Kurve) bei $x=0.4 \mathrm{~mm}$ für $1.6 \mathrm{~Hz}$ : (a) und (b): Betrag und Phase der normierten $\tilde{u}$ Komponente (c) und (d): Betrag und Phase der normierten $\tilde{v}$-Komponente 85

5.10 Vergleich der Nahfelder im Kompensationsexperiment $\tilde{\psi}_{N F}^{K o m}$ (blaue Kurve) und bei Überlagerung von $\tilde{\psi}_{N F}^{K l}+\tilde{\psi}_{N F}^{D r}=\tilde{\psi}_{N F}^{K l D r}$ (rote Kurve) bei $x=4 \mathrm{~mm}$ für $1.6 \mathrm{~Hz}$ : (a) und (b): Betrag und Phase der normierten $\tilde{u}$ Komponente (c) und (d): Betrag und Phase der normierten $\tilde{v}$-Komponente 86

5.11 Vergleich der Nahfelder im Kompensationsexperiment $\tilde{\psi}_{N F}^{K o m}$ (blaue Kurve) und bei Überlagerung von $\tilde{\psi}_{N F}^{K l}+\tilde{\psi}_{N F}^{D r}=\tilde{\psi}_{N F}^{K l D r}$ (rote Kurve) bei $x=8 \mathrm{~mm}$ für $1.6 \mathrm{~Hz}$ : (a) und (b): Betrag und Phase der normierten $\tilde{u}$ Komponente (c) und (d): Betrag und Phase der normierten $\tilde{v}$-Komponente 87

5.12 Betrag der scheinbaren Geschwindigkeit $\tilde{u}_{s}^{\text {Kom }}$ im Kompensationsfeld bei $1.6 \mathrm{~Hz} \ldots \ldots \ldots \ldots \ldots \ldots$

5.13 Vergleich der $\tilde{u}$-Komponenten der Nahfelder $\tilde{u}_{N F}^{D r}$ (rote Punkte), $\tilde{u}_{N F}^{K l}$ (blaue Punkte) und $\tilde{u}_{N F}^{K o m}$ (schwarze Punkte) mit den scheinbaren Geschwindigkeiten $\tilde{u}_{s}^{D r}$ (rote Kurve), $\tilde{u}_{s}^{K l}$ (blaue Kurve) und $\tilde{u}_{s}^{K o m}$ (schwarze Kurve) in Abhängigkeit von der $x$-Koordinate für $1.6 \mathrm{~Hz}$ : (a) Betrag, (b) Phase bei $y=0.4 \mathrm{~mm}$, (c) Betrag und (d) Phase bei $y=2 \mathrm{~mm}$. . . . .

5.14 Die mit $V_{k}$ und $d_{0}$ normierte Divergenz $\tilde{T}$ des scheinbaren Geschwindigkeitsfeldes in Abhängigkeit von der $x$-Koordinate für die drei verschiedenen Felder (Druckgradienten-Anregung $\tilde{T}^{D r}$ (rot), SchwingkantenAnregung $\tilde{T}^{K l}$ (blau) und Kompensationsfeld $\tilde{T}^{\text {Kom }}$ (schwarz) bei $1.6 \mathrm{~Hz}$

A.1 Die numerische Rechnung bei dem Konvektionsterm mittels der CIP-

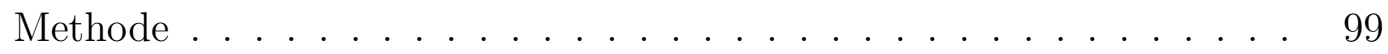

A.2 Das bei der numerischen Rechnung verwendete Gitter . . . . . . . . . 100

A.3 Das Modell der Anregungsvorrichtungen bei der numerischen Rechnung durch eine Verteilung von Quellen und Senken. Die linke Darstellung zeigt die Druckgradient-Anregung, die rechte Seite ist die Schwingkanten-Anregung. . . . . . . . . . . . . . . . . . 100

A.4 Das Flussdiagram der numerischen Rechnung mittels der CIP-Methode 102

A.5 Amplitude der Wechselgeschwindigkeit im Bereich der Plattenhinterkante bei Druckgradienten-Anregung mit $2 \mathrm{~Hz}$ : $\tilde{u}$-Komponente (oben) und $\tilde{v}$-Komponente (unten) . . . . . . . . . . . . . . . . 103 
A.6 Amplitude der Wechselgeschwindigkeit im Bereich der Plattenhinterkante bei Schwingkanten-Anregung mit $2 \mathrm{~Hz}$ : $\tilde{u}$-Komponente (oben) und $\tilde{v}$-Komponente (unten) . . . . . . . . . . . . . . . . . 104

A.7 Skizze zur Definition von $V_{S t}$ und $K_{A} \ldots \ldots \ldots \ldots \ldots$

A.8 Die schematische Darstellung der vier verschiedenen Schnitte zum Vergleich der numerischen Lösung mit dem Experiment . . . . . . . . . . . 106

A.9 Die numerischen Ergebnisse im Vergleich mit experimentellen Werten mit Druckgradienten-Anregung bei $2 \mathrm{~Hz}$ für vier verschiedene Schnitte (A), (B), (C) und (D) (siehe Abbildung A.8); Amplitude (links) und Phase (rechts), Punkte: Messung, Linien: Numerik . . . . . . . . . . . . 107

A.10 Die numerischen Ergebnisse im Vergleich mit experimentellen Werten mit Schwingkanten-Anregung bei $2 \mathrm{~Hz}$ für vier verschiedene Schnitte (A), (B), (C) und (D) (siehe Abbildung A.8); Amplitude (links) und Phase (rechts), Punkte: Messung, Linien: Numerik . . . . . . . . . . . . 108

A.11 Betrag (a) und Phase (b) der $\tilde{v}$-Komponente durch die DruckgradientenAnregung als Funktion von $x$ für vier verschiedene Anregungsfrequenzen bei $y=0 \mathrm{~mm} \ldots \ldots \ldots$. . . . . . . . . . . . . . . . 110

A.12 Betrag (a) und Phase (b) der $\tilde{v}$-Komponente durch die SchwingkantenAnregung als Funktion von $x$ für vier verschiedene Anregungsfrequenzen bei $y=0 \mathrm{~mm} \ldots \ldots \ldots \ldots$. . . . . . . . . . . . . . . . . . . . . 



\section{Verzeichnis wichtiger Symbole}

$\begin{array}{ll}C_{A} & \text { lokale Amplitude für die angefachte Mode der KH-Wellen } \\ C_{D} & \text { lokale Amplitude für die gedämpfte Mode der KH-Wellen } \\ d_{0} & \text { für anregende Felder bei ruhendem Medium charakteristische } \\ & \text { Länge (in der vorliegenden Arbeit } d_{0}=1 \text { mm) } \\ R_{\Theta}=U_{1} \Theta_{0} / \nu & \text { Reynoldszahl (gebildet mit der Impulsverlustdicke) } \\ S t_{\Theta}=\beta \Theta_{0} / U_{1} & \text { Strouhalzahl (gebildet mit der Impulsverlustdicke) } \\ \tilde{T} & \text { Divergenz von }\left(\tilde{u}_{s}, \tilde{v}_{s}\right) \text { (Def. s. Gl. (4.14) bzw. (5.8)) } \\ u, v & \text { Geschwindigkeitskomponenten bez. der } x \text { - und } y \text {-Richtung } \\ u_{\text {eff }}^{\prime} & \text { Effektivwert der tubulenten Fluktuationen } \\ U_{1}, U_{2} & \text { Geschwindigkeiten in der Haupt- und in der Nebenströmung } \\ & \text { im großen Abstand von der Scherschicht } \\ V_{k}=\left|\tilde{v}_{A n F}^{K l}(0,0)\right| & \text { Normierungsgröße bei der Schwingkanten-Anregung } \\ V_{n}=\left|\tilde{v}_{A n F}^{D r}\left(d_{0}, 0\right)\right| & \text { Normierungsgröße bei der Druckgradienten-Anregung } \\ \alpha & \text { komplexe Wellenzahl; Im\{ } \alpha\}<0, \text { Im }\left\{\alpha^{*}\right\}>0 \\ \beta=2 \pi f & \text { Kreisfrequenz der harmonischen Störung } \\ \delta_{S t}=\sqrt{2 \nu / \beta} & \text { Dicke der Stokesschicht } \\ \delta_{1} & \text { Verdrängungsdicke (Def. s. Gl. }(3.4)) \\ \eta & \text { Auslenkung des Teilchens in } y \text {-Richtung bei im Mittel ruhen- } \\ \Theta & \text { dem Medium } \\ \Theta_{0}=\left.\Theta\right|_{x=0} & \text { Impulsverlustdicke (Def. s. Gl. }(3.3)) \\ \nu & \text { Impulsverlustdicke bei } x=0 \text { zur Normierung } \\ \xi & \text { kinematische Zähigkeit } \\ \psi & \text { Auslenkung des Teilchens in } x \text {-Richtung bei im Mittel ruhen- } \\ (~ & \text { dem Medium } \\ ()^{K r} & \text { Stromfunktion }\end{array}$




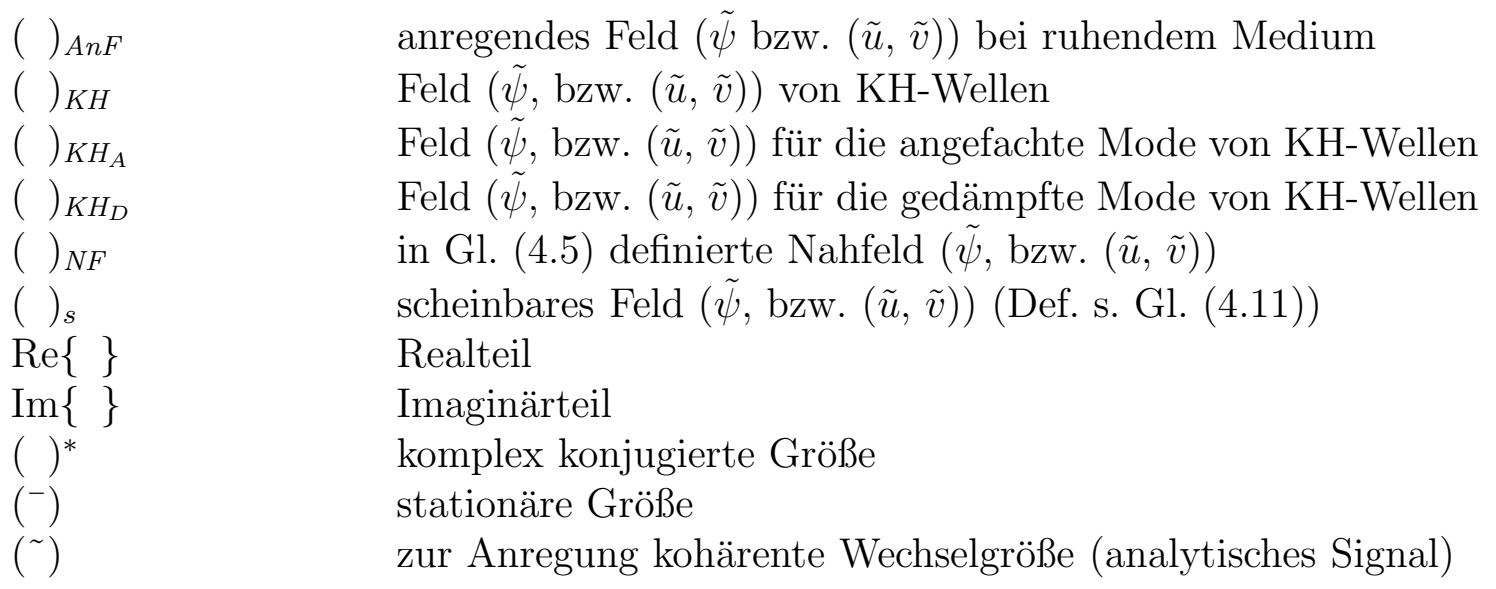




\section{Kapitel 1}

\section{Einleitung}

Treten bei überströmten Oberflächen fester Körper starke negative Krümmungen auf, so löst die Grenzschicht von der Körperoberfläche ab, da innerhalb der Grenzschicht die Strömungsgeschwindigkeit aufgrund des positiven Druckgradienten und der Wandreibung auf null abfällt. Bei dem Prozess der Ablösung geht die Wandgrenzschicht in eine freie Scherschicht über. Abgelösete freie Scherschichten bilden sich entsprechend hinter überströmten Kanten, z. B. von Tragflächen oder in durchströmten Kanälen. Die Dynamik der freien Scherschicht wird über einen weiten Frequenzbereich durch eine konvektive Instabilität, die sogenannte Kelvin-Helmholtz-Instabilität (KH-Instabilität) dominiert, bei der die Zähigkeit keine wesentliche Rolle spielt und die sich daher in guter Nährung durch die Euler-Gleichung beschreiben lässt.

Die Dynamik der Strömungsablösung bei instationären Strömungen ist dagegen sehr viel komplizierter, da sie sowohl durch reibungsfreie Kräfte als auch durch viskose Kräfte bestimmt ist. Da zeitlich schwankende Randbedingungen an der Ablösekante zur Anregung der KH-Instabilität in der Scherschicht führen, stehen die instationäre Strömungsablösung und die Anregbarkeit der KH-Instabilität im engen Zusammenhang. In diesem Sinne kann die vorliegende Untersuchung der Anregbarkeit der KHInstabilität als ein „Werkzeug“ zur Untersuchung der instationären Ablösung, die sowohl im Bereich der Strömungsakustik als auch der Aerodynamik von großer Bedeutung ist, verstanden werden. Die Schallentstehung oder Schallstreuung an der Ablösekante wie auch der instationäre Auftrieb sind Phänomene, die durch die Dynamik der Ablösung mitbestimmt werden.

Aber auch der eigentliche Anregungsmechanismus der KH-Instabilität selbst, der die Grundlage für die Rezeptivität der Scherschicht bildet, ist von Interesse. Wenn die Scherschicht auf ein Hindernis, z. B. eine weitere, stromab gelegene Kante trifft und die dort entstehenden Druckschwankungen auf die Ablösekante zurückkoppeln, kann Schall aus der Instabilität der Scherschicht entstehen. Die KH-Instabilität ist der für diese selbsterregten notwendige Verstärkungsmechanismus. Darüber hinaus spielt die Anregbarkeit der KH-Instabilität durch verschiedene Instationaritäten auch in der Steuerung von Strömungen eine wichtige Rolle, z. B., wenn Mischprozesse beschleunigt werden sollen oder wenn es um die Unterdrückung von Instabilitäten geht.

Zur Anregung der KH-Instabilität wurden in den vorgangen Jahren Erkenntnisse 
erzielt. Für unendlich dünne Scherschichten (d.h. für verschwindende Strouhalzahl $\left.S t_{\Theta}=0\right)$ geben BECHERT \& Michel [2] eine analytische Lösung an, die die von einer Monopol bzw. einer Dipolquelle angeregte Scherschicht, die von einer halbunendlichen Platte abgelöst ist, beschreibt. In weiteren Studien weisen BECHERT [3] bzw. BECHERT \& STAHL [5] an einer realen Scherschicht nach, dass die Instabilitätswellenanregung dieser analytischen Lösung bei kleinen Strouhalzahlen $\left(S t_{\Theta}<0.005\right)$ folgt, wenn man quasistationäre Strömungsablösung annimmt. Es ist allerdings nicht klar, wie diese Lösung auf endlich dicke Scherschichten und auf beliebige instationäre Randbedingungen erweitert werden kann.

REBEL [20] regt die KH-Instabilität einerseits durch eine in der Grenzschicht der Splitterplatte erzeugte Tollmien-Schlichtung-Welle (TS-Welle), andererseits durch Druckgradientenfelder an, die mit schwingenden Membranen in den Seitenwänden des Strömungskanals erzeugt werden. Er zeigte, dass eine über die Kante propagierende TSWelle, in der die Wirbeligkeit schon ähnlich wie in der resultierenden KH-Instabilität verteilt ist, zu kleineren Amplituden der KH-Instabilität führt als das durch die Membranen erzeugte Druckgradientenfeld.

DIERKEN [8] verwendet einen dünnen Zylinder zur Anregung der KH-Instabilität, der die Hinterkante der Platte bildet und zeitlich sinusförmig um seine Achse rotiert. Er überlagerte zwei Anregungsmechanismen (Zylinder- und Druckgradienten-Anregung) so, dass die resultierende KH-Instabilität verschwindet. Es zeigt sich aus dieser Untersuchung, dass im kompensierten Feld die Scherrate $\partial \tilde{v} / \partial x(\tilde{v}=$ Normalschnelle $)$ hinter der Kante (außerhalb der Stokesschicht) klein wird. Außerdem wurde dabei gezeigt, dass nicht die an der Oberfläche des Zylinder erzeugte wirbelbehaftete Stokesschicht sondern die wirbelfreie Rückströmung für die Anregung der KH-Instabilität maßgebend ist.

Mit Blick auf die Dynamik der Strömungsablösung stellt Graf [13] ein Modell vor, das die Strömungsablösung als Wirbeltransport-Problem beschreibt. Das Modell unterteilt den Wirbeltransport in mehrere Einzelprozesse: Die Erzeugung von Wirbeligkeit an der Plattenoberfläche durch einen aufgeprägten Druckgradienten, die ebenfalls durch den Druckgradienten ausgelöste Auslenkung der als dünn angenommenen ablösenden Wirbeligkeitsschicht und der Transport der erzeugten Wirbeligkeit in die Scherschicht durch Diffusion und Konvektion. Diese Betrachtung liefert eine Abhängigkeit der charakteristischen Zeitskalen von der Diffusions- und Konvektionszeit der Strömung. Anhand von experimentellen Untersuchungen (GRAF [13]) und anhand von numerischen Simulation (BEBBER [1]) konnte dieses Wirbelmodell bis zu gewissen Grade nachvollzogen werden. SCHMITz [23] setzt diese Untersuchung mit einer numerischen Methode fort.

In dieser Arbeit wird die Anregbarkeit der KH-Instabilität mittels zweier Anregungsvorrichtungen experimentell untersucht, um eine maßgebende Größe im Nahfeld zu finden, auf die man die Amplitude der KH-Instabilität beziehen kann. Zu diesem Zweck wird zuerst der für die Untersuchung verwendete Wasserkanal dargestellt, in dem sich eine überströmte Splitterplatte und zwei verschiedene Anregungsvorrichtungen befinden (Kapitel 2). Die Gleichströmung wird zunächst in Kapitel 3 betrachtet. 
In Kapitel 4 wird die Wechselwirkung zwischen der Gleichströmung und der reinen Wechselströmung untersucht (die numerische Berechnung der reinen Wechselströmung ohne Gleichströmungsüberlagerung sowie der Vergleich mit experimentellen Ergebnissen ist in Anhang A dargestellt). Dabei gelingt es, das gemessene Wechselfeld in drei Anteile zu zerlegen: das anregende Feld selbst, das Feld der KH-Instabilität und das Nahfeld, das nicht zu den ersten zwei Feldern gehört. Ziel dieses Kapitels ist die Bestimmung dieses Nahfeldes. In Kapitel 5 wird das im Kapitel 4 bestimmte Nahfeld mittels eines Kompensationsexperimentes untersucht, das durch die Ergebnisse von DIERKEN motiviert war. Das Kompensationsexperiment stellt eine interessante Untersuchungsmethode dar, da auf diese Weise ein nichtanregendes Nahfeld erzeugt werden kann. 



\section{Kapitel 2}

\section{Der experimentelle Aufbau und das Messverfahren}

In diesem Kapitel werden der Aufbau des Strömungskanals sowie die zwei verschiedenen Anregungsvorrichtungen, die sich in der Messstrecke des Kanals befinden, vorgestellt. Es wird außerdem auf das verwendete Messverfahren der Laser-Doppler-Anemometrie sowie die computergesteuerte Datenaufnahme und Weiterverarbeitung eingegangen.

\subsection{Der Wasserkanal}

Für die Untersuchungen des Projektes wurde ein spezieller, möglichst störungsarmer Wasserkanal aufgebaut. Ziel war die Präparation einer ebenen, laminaren Wandgrenzschicht bei kleinen Strömungsgeschwindigkeiten, um die zu untersuchenden instationären Strömungsvorgänge mit dem Auge beobachten zu können. Wasser eignet sich für diese Zwecke besser als Luft, da die langsame Strömung problemloser sichtbar gemacht werden kann und thermische Konvektionseffekte viel einfacher ausgeschlossen werden können. Dieser Wasserkanal wurde schon von Rebel [20], GrützMacher [14], Dierken [8] , Enghardt [9], und Graf [13] verwendet und ist dort ausführlich beschrieben.

Die Strömung im Kanal war dabei zeitlich sehr konstant, wies aber bei den drei ersten Untersuchungen noch eine starke unerwünschte Abhängigkeit der Geschwindigkeit von der Spannweitenrichtung auf, so dass die Strömung nicht als völlig zweidimensional angesehen werden konnte. Dies wurde durch den Umbau des Kanals verbessert, der von ENGHARDT [9] durchgeführt wurde, und auch dort beschrieben ist.

Abbildung 2.1 zeigt eine Übersichtskizze des Wasserkanals, an Hand derer zunächst die Funktionsweise des Kanals erläutert werden soll. Der Kanal mit rechteckigem Querschnitt ist vertikal aufgebaut und wird durch Schwerkraft angetrieben, womit Langzeitschwankungen des Antriebs vermieden werden. Das ist für Versuchsreihen, die sich über mehrere Wochen hinziehen, notwendig.

Der größte Teil des Wasserkanals (bis auf den Einlauftrichter sowie das Überlaufbecken) und die Messstrecke sind aus Plexiglas gefertigt und ermöglichen so die optische Messwertaufnahme durch Laser-Doppler-Anemometrie und zusätzlich die einfache Beobach- 


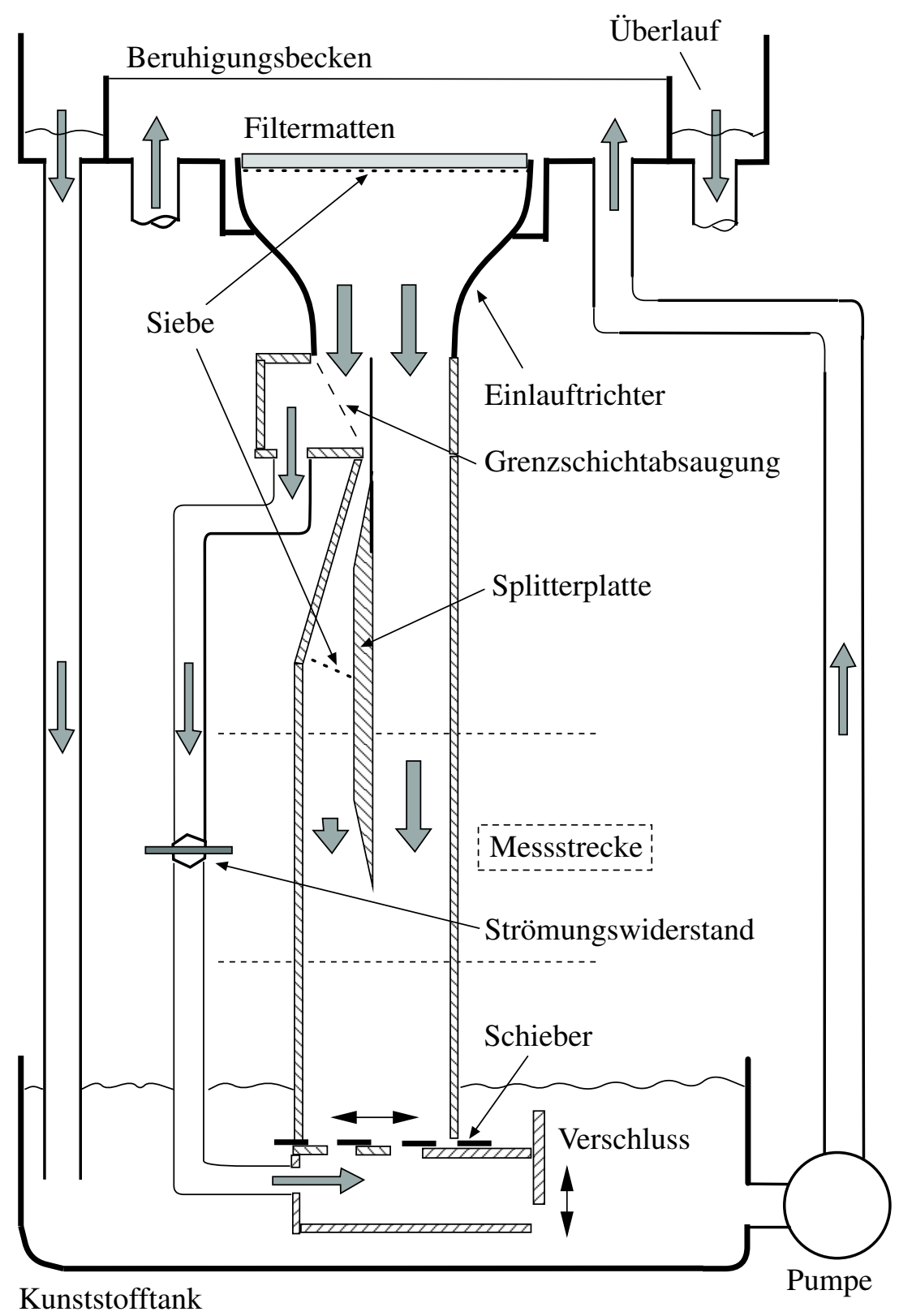

Abb. 2.1: Der bei der Messung verwendete Wasserkanal 
tung der Strömung durch Tintenfäden.

Während des Betriebs wird Wasser mit einer Kreiselpumpe aus einem 350l fassenden Kunststofftank ins Beruhigungsbecken gepumpt. Die Fördermenge der Pumpe wird dabei so eingestellt, dass das Beruhigungsbecken stets ein wenig überläuft. Der Überlauf dient dazu, den Wasserstand und damit die Antriebskraft der Strömung konstant zu halten. Vor dem Einlauftrichter befinden sich zwei Filtermatten, die auf dem sehr feinmaschigen Sieb oberhalb des Trichters aufeinander liegen. Der Einlauftrichter mit rechteckigem Querschnitt und einem Kontraktionsverhältnis von 1 : 11.6 sowie einem praktisch konstant gehaltenen Seitenverhältnis wurde so konzipiert, dass jede Strömungsablösung (insbesondere in den Ecken) vermieden wird und das Strömungsprofil im Auslauf sehr eben ist.

Am Ende des Einlauftrichters trifft die Strömung auf die Vorderkante der Splitterplatte. Dabei wird die Trichtergrenzschicht an drei Seiten abgeschält. Nur die Wandgrenzschicht, die der Splitterplatte gegenüberliegt, wird nicht erneuert. Über ein Rohr wird das Wasser in den Tank zurückgeführt. Mit einem Strömungswiderstand wird die Absaugung so eingestellt, dass an der Vorderkante der Splitterplatte keine Querströmung auftritt, die in der Messstrecke zur Abhängigkeit der Geschwindigkeit von der Spannweitenrichtung führen würde. Der Aufbau des Trichters und der Grenzschichtabsaugung an drei Seiten des Kanals wurden im Detail bei EnghardT [9] beschrieben.

Ein kleiner Teil der abgesaugten Strömung wird durch einen $6 \mathrm{~mm}$ breiten Schlitz und ein engmaschiges Sieb in den Nebenkanal geleitet. Die Nebenströmung ist eine langsame Spülströmung, die verhindert, dass sich an der Hinterkante der Splitterplatte großräumige Wirbel ausbilden können. In der Hauptströmung, auf der anderen Seite der ca. $910 \mathrm{~mm}$ langen Splitterplatte bildet sich eine laminare Grenzschicht aus. Die Haupt- und die Nebenströmung treffen sich an der Hinterkante wieder, und dort bildet sich eine laminare Scherschicht aus. Die Dynamik der freien Scherschicht wird durch konvektive Instabilitätswellen, sogenannte Kelvin-Helmholtz-Instabilitätswellen (KHWellen), dominiert, die durch kleine Störungen angeregt werden.

Der Kanal ist für eine Hauptströmungsgeschwindigkeit von $U_{1}=15 \mathrm{~cm} / \mathrm{s}$ ausgelegt. Bei dieser Geschwindigkeit, bei der auch die Messungen durchgeführt wurden, ist die Strömung besonders homogen und zeitlich konstant. Die Nebenströmungsgeschwindigkeit beträgt ca. 10\% der Strömungsgeschwindigkeit in der Hauptströmung. Mit Hilfe des Schiebers lässt sich die Strömungsgeschwindigkeit bei geeigneter Anpassung der Pumpenfördermenge variieren.

Wegen der von der Kreiselpumpe abgegebenen Leistung stellt sich die Wassertemperatur ohne Kühlung auf einen Wert von ca. $26^{\circ}$ ein, hängt dabei aber wegen der freien Wasseroberflächen im Beruhigungsbecken und im Kunststofftank stark von den Lufttemperaturschwankungen im Labor ab. Das Wasser muss daher gekühlt werden, um die Wassertemperatur konstant zu halten. Die Kühlung ist durch einen über einen Wärmetauscher mit dem Kanal verbundenen Kühlkreislauf realisiert. Ein Kühlaggregat, das die Vorlauftemperatur des Kühlkreislaufes vorgibt, wird über einen Wassertemperatur- sowie über zwei Lufttemperaturfühler so geregelt, dass die Temperaturabweichungen unter $\pm 0.03^{\circ}$ bleiben (siehe Abbildung 2.2). 


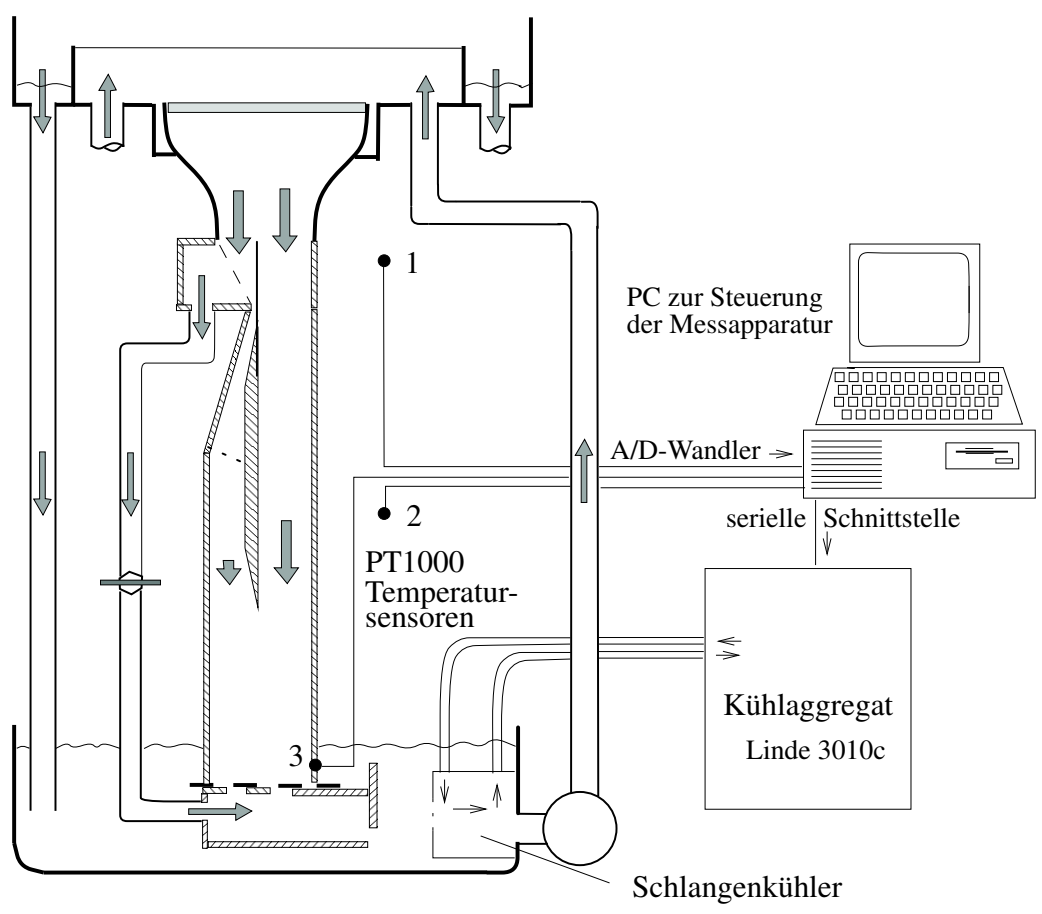

Abb. 2.2: Schematischer Aufbau des Temperaturregelkreises im Wasserkanal

\subsection{Die Anregungsvorrichtungen}

Abbildung 2.3 zeigt die Messstrecke des Kanals, in der die Messungen durchgeführt werden. Die Abbildung ist so gedreht, dass die Hauptströmung von links kommt. Das rechtwinklige Koordinatensystem liegt so im Kanal, dass die $x$-Richtung mit der Hauptströmungsrichtung zusammenfällt. Die $y$-Richtung ist wie üblich die Richtung normal zur Plattenoberfläche. Der Koordinatenursprung wurde so gewählt, dass $x=0$ mit der Splitterplattenhinterkante übereinstimmt, $y=0$ auf der Splitterplattenoberfläche und $z=0$ in der Kanalmitte liegt.

Da die vorliegene Arbeit von der Anregung der KH-Instabilitätswelle handelt, ist es sehr wichtig, womit die in der Messstrecke entstehende freie Scherschicht künstlich angeregt wird. Hier werden nun zwei verschiedene Anregungusvorrichtungen vorgestellt, die sich in der Messstrecke befinden.

\subsubsection{Die Druckgradienten-Anregung in den Seitenwänden des Kanals}

Zwei Membranen, die in der Seitenwänden integriert sind, können sich gleichphasig, sinusförmig bewegen und einen Druckgradient senkrecht zur Plattenoberfäche erzeugen. Diese Druckgradienten-Anregung wurde im Prinzip schon von GrützMacher [14] für seine Experimente zur Unterdrückung der Instabilitätswellen verwendet, der Antrieb aber von DiERKEN [8] erneuert. Er besteht aus einer schrittmotorgetriebe- 


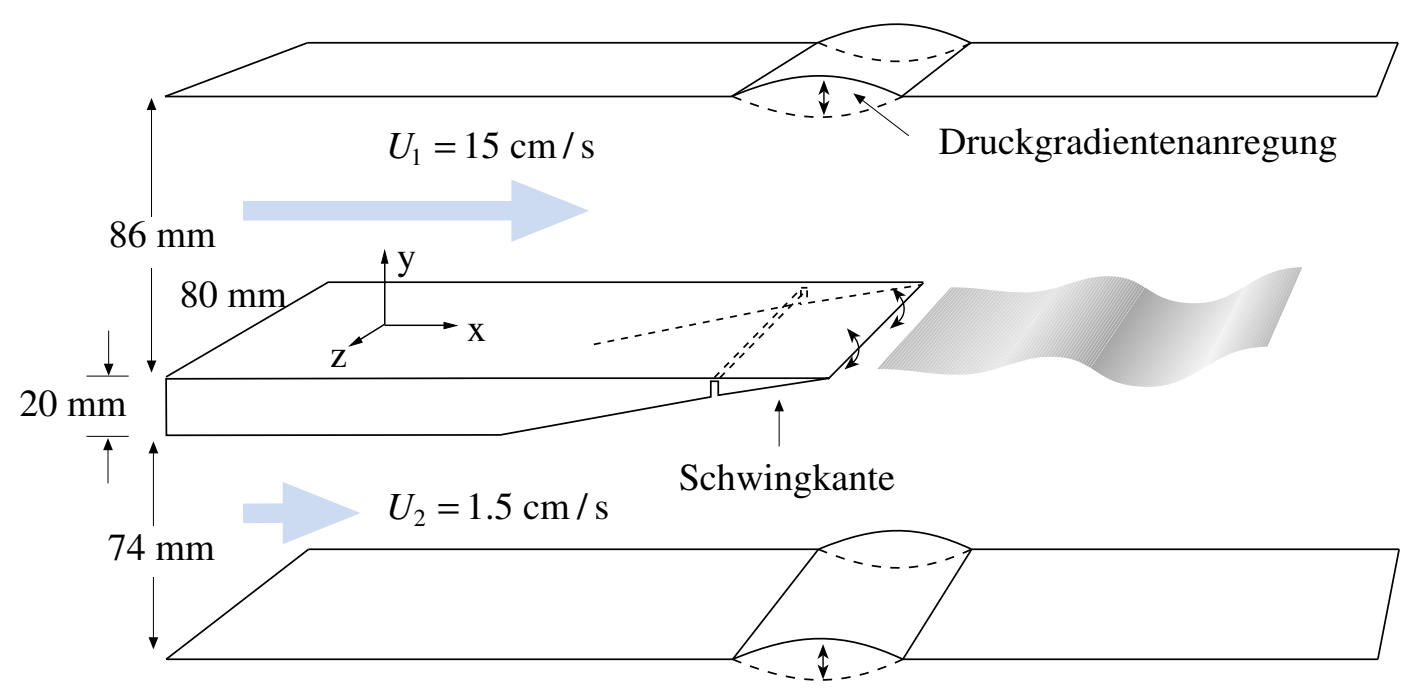

Abb. 2.3: Messstrecke des Wasserkanals mit der Druckgradienten-Anregung bzw. mit der Schwingkante

nen Mechanik. Die Drehbewegung des Motors wird mittels einer Kugelgewindespindel mit einer Steigung von $2.5 \mathrm{~mm}$ pro Umdrehung auf einen doppelt wirkenden Kolben übertragen, der in einem Zylinder zwei entgegengesetzt gleiche Volumenverschiebungen hervorruft. Durch wassergefüllte Anschlussleitungen werden diese auf die Membranen in den Kanalwänden übertragen, die dann einen Druckgradient im Kanal erzeugen. Der effektive Querschnitt des Zylinders beträgt $150.8 \mathrm{~mm}^{2}$. Durch die ausgewählte Kombination des Schrittmotors und der Kugelgewindespindel kann die Linearbewegung der Volumenverschiebung mit einer Auflösung von $0.000625 \mathrm{~mm}$ vorgegeben werden. Die Trägheitskräfte der Mechanik wachsen quadratisch mit der Frequenz, und verursachen so eine Obergrenze des ausnutzbaren Frequenzbereichs von $6 \mathrm{~Hz}$.

\subsubsection{Die lokale Anregung durch eine Schwinghinterkante}

Es zeigte sich bei der Untersuchung von DiERKEN [8], dass ein dünner Zylinder (2 mm Durchmesser), der die Hinterkante der Platte bildet und zeitlich sinusförmig um seine Achse rotiert, eine lokale Störung erzeugt und dass diese Störung zur Anregung der Instabilitätswelle führt. Als wichtige Erkenntnis ergab sich aus dieser Untersuchung:

- Die an der Oberfläche des Zylinders erzeugte wirbelbehaftete Stokesschicht spielt keine unmittelbare Rolle bei der Anregung der KH-Welle, sondern die wirbelfreie Rückströmung, die durch Abschälen der Stokesschicht an dem Übergang zur festen Platte erzeugt wird, ist dabei maßgebend.

- Im Vergleich mit der Druckgradienten-Anregung regt die Zylinderanregung die KH-Welle verzögert an.

Diese Erkentnisse führen zur Idee einer neuen lokalen Anregungsvorrichtung, die noch effektiver Instabilitätswellen anregen kann als die Zylinderanregung. Da die 


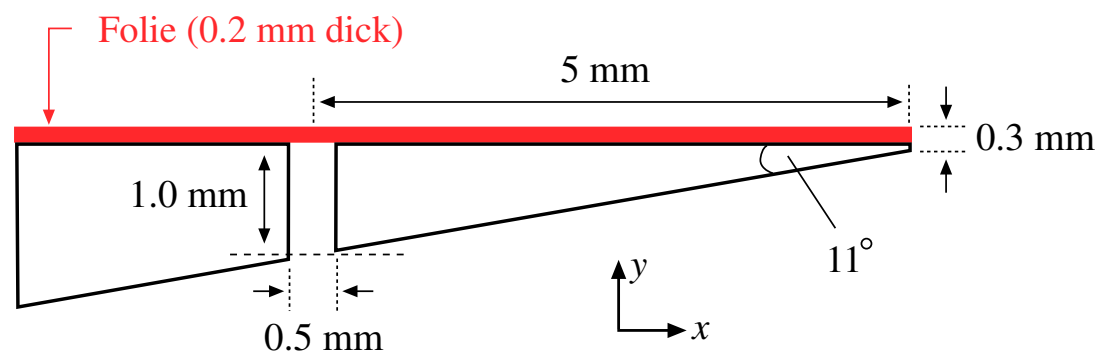

Abb. 2.4: Die verwendete Splitterplatte mit schwingender Hinterkante

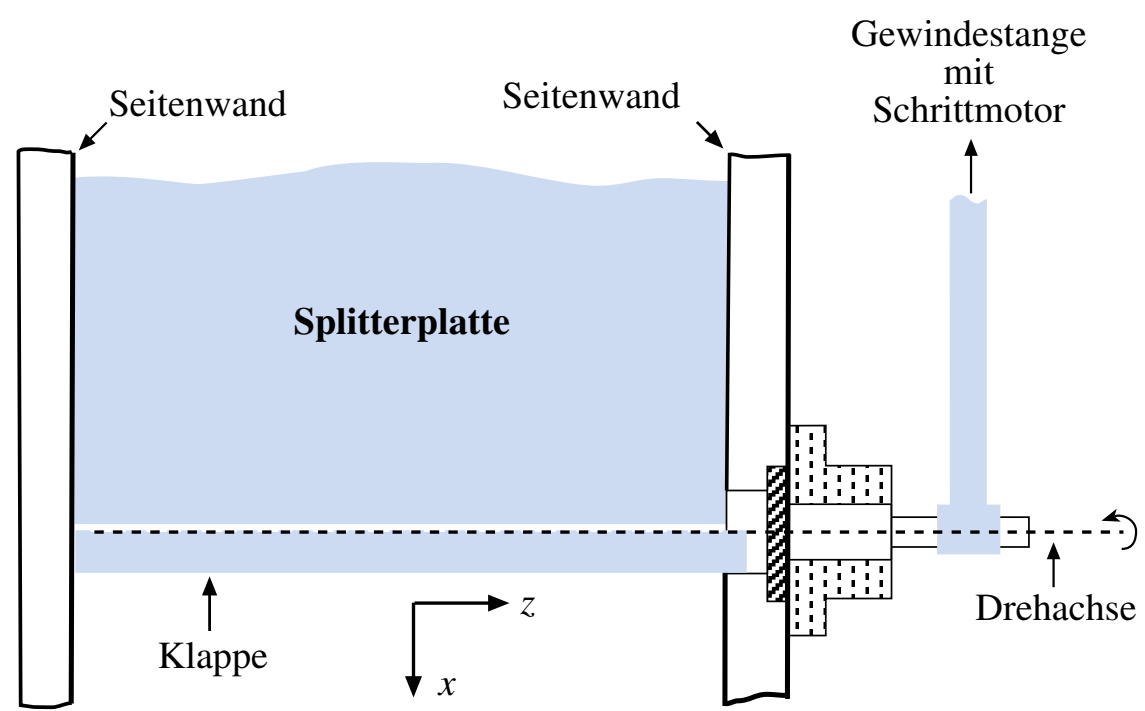

Abb. 2.5: Seitenansicht der Schwingkanten-Anregung

Rückströmung der Zylinderanregung durch das Abschälen der Stokesschicht zwischen der Zylinderrotation und der Hinterkante der Splitterplatte verursacht wird, kann man das Abschälen vereinfachend als eine Dipolquelle dargestellen.

Eine Dipolstörung an der Hinterkante kann noch effektiver erzeugt werden, wenn die Quellen und Senken in einem endlichen Bereich an der Hinterkante verteilt werden. Dies wurde durch eine Klappe realisiert, die die Hinterkante bildet und in einem kleinen Winkelbereich ausgelenkt werden kann. Abbildung 2.4 und 2.5 stellen die auslenkbare Hinterkante und deren Ansteuerung dar.

Der Antrieb der Schwingkante besteht auch aus einer schrittmotorgetriebenen Mechanik. Die Resonanzfrequenzen des verwendeten Motors liegen bei ca. $200 \mathrm{~Hz}$ und liegen damit weit außerhalb des hier betrachteten Frequenzbereichs. Die Drehbewegung des Schrittmotors wird über eine Kugelgewindespindel mit einer Steigung von $2 \mathrm{~mm}$ pro Umdrehung auf einen Hebel mit einer Länge $275 \mathrm{~mm}$ übertragen. Anschließend dreht sich eine Führungswelle auf der Drehachse der Klappe, die über ein Kugellager an der Kanalwand befestigt ist. Mit Hilfe der Kugelgewindespindel und des Hebels können 

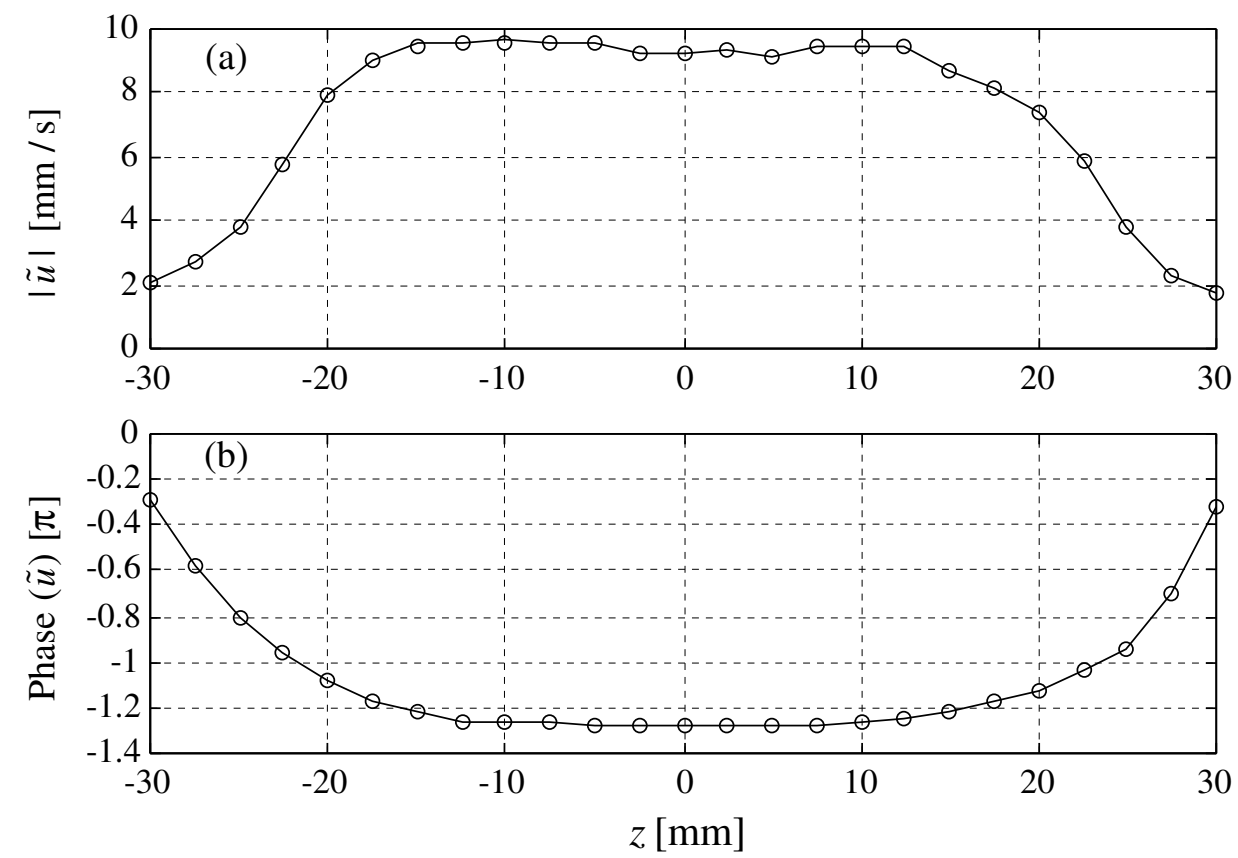

Abb. 2.6: Die durch die schwingende Hinterkante angeregte Wechselgeschwindigkeit ( $u$-Komponente) in Abhängigkeit von der $z$-Koordinate bei $1.6 \mathrm{~Hz}$, wobei $U_{1}=15 \mathrm{~cm} / \mathrm{s}$, $x=30 \mathrm{~mm}$ und $y=2 \mathrm{~mm}$ : (a) der Betrag und (b) die Phase

Drehbewegungen der Hinterkante mit einer Aufösung von ca. $0.001^{\circ}$ realisiert werden. Der Bau der Anregung führt insbesondere im Bereich der bewegbaren Hinterkante auf große feinmechanische Probleme, da die $5 \mathrm{~mm}$ lange Hinterkante auf einer freien Länge von $80 \mathrm{~mm}$ mit konstanter Amplitude und Phase schwingen soll. Abbildung 2.4 zeigt die nach einigen Vorversuchen gewählte Konstruktion.

Die beiden Seitenflächen der Splitterplatte laufen unter einem Winkel von etwa $11^{\circ}$ aufeinander zu. Das Ende der Splitterplatte wurde bei $x=-5.25 \mathrm{~mm}$ senkrecht zur $x$ Koordinate abgeschnitten. Zwischen der beweglichen Hinterkante und dem festen Teil der Splitterplatte wurde dann ein $0.5 \mathrm{~mm}$ breiter Spalt gelassen. Mittels einer doppelt aufgebrachten, selbstklebenden Folie wurden die beiden Teile so miteinander verbunden, dass zum einen die Spaltbreite über der gesamten Länge konstant ist, zum anderen Knicke auf der Oberfläche der Splitterplatte aufgrund der Schwingung der Hinterkante vermieden werden, die sonst Störungen in der Grenzschicht verursachen könnten. Da die Dicke einer einzelnen Folie $0.1 \mathrm{~mm}$ beträgt und das Ende der Hinterkante selbst eine endliche Dicke hat, beträgt anschließend die Dicke der schwingenden Hinterkante ca. $0.3 \mathrm{~mm}$.

Ob die Hinterkante tatsächlich entlang der $z$-Koordinate gleichmäßig schwingt, muss nun festgestellt werden. Für diese Zwecke wurden Wechselgeschwindigkeiten mit Überlagerung einer Gleichströmung $U_{1}=15 \mathrm{~cm} / \mathrm{s}$ in der Scherschicht bei $x=30 \mathrm{~mm}$ und $y=2 \mathrm{~mm}$ aufgenommen, die durch diese schwingende Hinterkante angeregt wurden. Abbildung 2.6 zeigt die Abhängigkeit der $u$-Komponente von der Spannweitenkoordinate $z$, wobei die Hinterkante mit einer Frequenz von $1.6 \mathrm{~Hz}$ geschwungen hat. Aus 
Kapitel 2. Der experimentelle Aufbau und das Messverfahren

der Auftragung ist zu erkennen, dass sich die Instabilitätswellen sowohl hinsichtlich des Betrages als auch der Phase im Bereich $-10 \mathrm{~mm}<z<10 \mathrm{~mm}$ gleichmäßig ausbreiten. Es lässt sich daher annehmen, dass die Hinterkante in diesem Bereich sehr homogen schwingt.

Die Ansteuerung des Schrittmotors wurde von Dierken übernommen. Mit Hilfe der von Steinhoff und Mitarbeitern (Steinhoff, Ebrecht \& Geiling [25]) entwickelten Schrittmotoransteuereinheit können zwei verschiedene Anregungsvorrichtungen (Druckgradienten- und Schwingkanten-Anregung in der vorliegenden Arbeit) parallel mit gleicher Frequenz und definierten Amplituden sowie definierter Phasendifferenz betrieben werden.

\subsection{Die Messdatenerfassung}

Die Erfassung der Messdaten mit Hilfe eines einkomponentigen Laser-DopplerAnemometers (LDA) sowie der Ablauf der Messung erfolgen rechnergesteuert und sind weitgehend automatisiert. Dies ist wichtig, da die Messungen manchmal über mehrere Tage laufen. Der Aufbau des LDA zur Messung des Geschwindigkeitsfeldes geht auf SchaAf [22] zurück, die Datenerfassung und das Programm, das den Messablauf steuert, wurden im wesentlichen von NEUWALD [17] entwickelt und dort ausführlich beschrieben. Hier sollen aus Verständnisgründen nur die wichtigsten Punkte noch einmal kurz zusammengefasst werden.

\subsubsection{LDA-Messung}

Für die LDA-Messung wird ein kommerzielles System (DANTEC 55X) verwendet, das die Messung von nur einer Geschwindigkeits-Komponente gestattet. Es besteht aus einem Helium-Neon-Laser mit einer Leistung von $18 \mathrm{~mW}$, dessen Wellenlänge $\lambda_{L}=632.8 \mathrm{~nm}$ ist, einem Strahlteiler, einer Braggzelle, der Empfangsoptik mit Photomultiplier und der dazugehörigen Frequenzverschiebeeinheit sowie einem Computerprozessor.

Die Strömungsgeschwindigkeit wird mit Hilfe zweier kohärenter Laserstrahlen bestimmt. Zuerst wird ein Laserstrahl mit Hilfe des Strahlteilers in zwei Teilstrahlen aufgeteilt. Durch eine Sammellinse mit einer Brennweite von $310 \mathrm{~mm}$ werden sie dann unter einem Winkel $2 \phi_{L}=11^{\circ}$ zur Überlagerung gebracht. Man erhält so das beim Kreuz-Strahlverfahren entstehende Schnittvolumen, das gleich dem Messvolumen ist. Das Messvolumen lässt sich unter Annahme gaußförmiger Strahlprofile als Rotationsellipsoid beschreiben mit einem Durchmesser von $d_{x}=d_{y}=0.2 \mathrm{~mm}$ und der Länge $d_{z}=2.2 \mathrm{~mm}$. Der Abstand der Interferenzstreifen im Schnittvolumen beträgt im vorliegenden Fall $\Delta x($ oder $\Delta y)=\lambda_{L} /\left(2 \sin \phi_{L}\right)=3.3 \mu \mathrm{m}$.

Fließen nun Streuteilchen (die in entgastem Leitungswasser hinreichend vorhanden sind) durch dieses Messvolumen, so wird die Intensität des gestreuten Lichts aufgrund der Interferenzebenen moduliert. Die Geschwindigkeitskomponente des Streuteilchens senkrecht zu den Interferenzebenen $u_{\perp}$ ist dabei durch diese Modulationsfrequenz $f_{M}$ bestimmt: $u_{\perp}=f_{M} \cdot \Delta x$. Weil diese Intensitätsmodulation keine Information über die 
Bewegungsrichtung der Streuteilchen enthält, wird die Frequenz eines der Laserstrahlen mit Hilfe einer Braggzelle optoakustisch um $40 \mathrm{MHz}$ verschoben, wodurch sich die Intensitätsstreifen im Schnittvolumen der Laserstrahlen mit einer Geschwindigkeit von $-131.2 \mathrm{~m} / \mathrm{s}$ bewegen.

Im Photomultiplier, dessen Empfangsoptik auf das Messvolumen fokussiert ist, wird das Streulicht in einen modulierten Strom umgesetzt. Der zeitliche Ablauf des Fotostroms stellt dabei einen Gaußimpuls dar, dem eine Modulation aufgeprägt ist. Das vom Photomultiplier kommende Signal wird dann von einer Frequenzverschiebeeinheit um $39.99 \mathrm{MHz}$ heruntergemischt, womit sich die effektive Geschwindigkeitsverschiebung auf $-32.8 \mathrm{~mm} / \mathrm{s}$ reduziert.

Im Counterprozessor durch Hochpassfilterung werden die tieffrequenten Anteile der Signale unterdrückt. Die Geschwindigkeit in negativer Richtung ist nun schließlich auf $u_{\perp}=-26.2 \mathrm{~mm} / \mathrm{s}$ begrenzt. Im Counterprozessor wird das Signal verstärkt und die Durchgangszeit des Teilchens durch acht Interferenzstreifen bestimmt. Nach einem digitalen Validitätstest wird der momentane Geschwindigkeitswert zusammen mit der Momentanphase der anregenden Schwingung in einen Datenpuffer gelegt, bis diese Werte vom PC eingelesen werden. Die mit der Anregungsfrequenz mitlaufende Phase wird dabei als 8-Bit-Zahl (Phasenauflösung $\pi / 128$ ) dargestellt.

$\underline{\text { Positionierung des Messvolumens }}$

Die optischen Komponenten des LDA einschließlich des Lasers und des Photomultipliers, sind auf einem gemeinsamen Verfahrtisch montiert, der sich mittels Schrittmotoren in alle drei Raumrichtungen bewegen kann. Die Ansteuerung erfolgt vom PC über eine serielle Schnittstelle und ein Interface, das dem Motor die gewünschte Drehrichtung sowie Schrittzahl mitteilt. Ein zweidimensionales Messfeld wird am PC eingegeben und dann automatisch abgefahren.

Fehlerquellen bei der LDA-Messung

Im folgenden sollen die wichtigsten Quellen systematischer Fehler bei der Erfassung der Geschwindigikeit mittels LDA diskutiert werden.

- Die Auflösung der Messwerte ist durch die Form der digitalen Repräsentation am Ausgang des Counterprozessors begrenzt: Eine 10 Bit breite Mantisse und ein 4 Bit breiter Exponent zur Basis 2 stehen zur Verfügung. Daduruch beträgt die Darstellungsgenauigkeit 0.1 bis $0.2 \%$ des Geschwindigkeitswertes, abhängig von der Frequenzverschiebung. Da die gemessene Geschwindigkeit um den durch die Frequenzverschiebung entstandenen Anteil größer ist als die tatsächliche Geschwindigkeit des Wassers, sollte diese Geschwindigkeitsverschiebung nicht zu groß gewählt werden, um das Digitalisierungsrauschen möglichst gering zu halten. Bei einer effektiven Frequenzverschiebung von $10 \mathrm{kHz}$ und bei einer Strömungsgeschwindigkeit von $15 \mathrm{~cm} / \mathrm{s}$ beträgt die kleinste aufösbare Geschwindigkeitsdifferenz in der Hauptströmungsrichtung etwa $0.2 \mathrm{~mm} / \mathrm{s}$. Für Messungen im Nebenströmungsbereich bzw. für Messungen der $v$-Komponente, bei denen 
man insgesamt kleinere Messwerte erhält, ist der Fehler entsprechend um eine Größenordnung kleiner.

- Ein weiterer unvermeidbarer Fehler entsteht durch die endliche Ausdehnung des Messvolumens. Die gemessene Geschwindigkeit ist vom Ort des Streuteilchens im Messvolumen abhängig, wenn die Geschwindigkeit des Wassers im Messvolumen nicht konstant ist (wie z.B. in der Scherschicht und der Plattengrenzschicht bei Messung der $u$-Komponente). Eine maximale Abweichung von der mittleren Strömungsgeschwindigkeit am Messpunkt (z.B. für die $u$-Komponente $\left.\Delta u_{\max }\right)$ erhält man durch folgende Abschätzung, wenn $u$ unabhängig von der $z$-Koordinate angenommen wird.

$$
\Delta u_{\max }=\sqrt{\left(\frac{\partial u}{\partial x} \cdot \frac{d_{x}}{2}\right)^{2}+\left(\frac{\partial u}{\partial y} \cdot \frac{d_{y}}{2}\right)^{2}}
$$

Da die Anzahl der gemessenen Geschwindigkeitswerte mit der Anzahl der sich durch das Messvolumen bewegenden Streuteilchen und deren Geschwindigkeit wächst, werden im Bereich großer Strömungsgeschwindigkeiten im gleichen Zeitraum mehr Geschwindigkeitswerte aufgenommen, als im Bereich kleiner Geschwindigkeiten. Diese Gewichtung ergibt sich auch innerhalb des Messvolumens. Da die maximal innerhalb des Messvolumens auftretenden Frequenzendifferenzen wegen der Frequenzverschiebung um eine Größordnung kleiner sind als die gemessenen Frequenzen selbst, und damit die systematische Verschiebung der Messwerte aufgrund des beschriebenen Effekts um mindestens zwei Größordnung kleiner sind als die tatsächliche Geschwindigkeit der Strömung, ist allerdings die Anhebung der mittleren gemessenen Geschwindigkeit als gering anzusehen.

- Bei der Justierung der Koordinaten ergibt sich ein weiteres Problem. Das Koordinatensystem des Verfahrtisches, mit dem das Messvolumen positioniert wird, muss sehr genau zum Kanalkoordinatensystem ausgerichtet werden. In der vorliegenen Arbeit wurden Messgitter um die Hinterkante verwendet, in denen Gitterpunkte nur $0.1 \mathrm{~mm}$ auseinanderliegen. Die Justiergenauigkeit des Messvolumens relativ zur Hinterkante beträgt aber nur $\pm 0.05 \mathrm{~mm}$, allerdings kann der Abstand der Gitterpunkte relativ zueinander sehr gut eingehalten werden. Um die Stelle zu finden, wo $y=0$ ist (auf der Splitterplatte), werden Messungen der $u$-Komponente in der Grenzschicht der Splitterplatte durchgeführt. Die Extrapolation der Messwerte auf die Geschwindigkeit Null liefert dann sowohl die Position $y=0$ als auch die Korrektur der Verdrehung zwischen dem Mess- und Kanalkoordinatensystem um die $x$ - und $z$-Achse. Eine Drehung des Verfahrtisches um die $y$-Achse muss mit Hilfe einer Wasserwaage vermieden werden. Da mehr als zwei Felder für die zwei verschiedenen Komponenten $u$ und $v$ jeweils für zwei Anregungsvorrichtungen nacheinander ausgemessen werden, liegt zwischen den zwei Messungen für die beiden Komponenten $u$ und $v$ am selben Messort $(x, y)$ eine längere Zeit und vor allem ein längerer Weg. Die Schrittmotoren verlieren 
bei längeren Wegen manchmal Schritte, daher sollte der Nullpunkt des Verfahrtisches jeweils nach der Messung eines Teilfeldes von ca. 600 Punkten neu jusitiert werden. Diese Justierung wird mit Hilfe einer Sammmellinse, die von GRAF [13] oberhalb des Messbereiches am Kanal aufgebaut wurde, durchgeführt. Sie bildet das Messvolumen auf eine ca. $1 \mathrm{~m}$ entfernte Wand ab. Dort wird nun das Bild wieder mit der Sollposition in Deckung gebracht, und die Koordinaten werden übernommen.

\subsubsection{Anpassung der Messwerte mittels phasenstarrer Mitte- lung}

Um die Dynamik der sich ablösenden freien Scherschicht zu untersuchen, wird der Strömung durch die Schwingkante bzw. durch die Druckgradientanregung eine Störung aufgeprägt. Die experimentelle Methode besteht darin, die Antwort des Strömungsfeldes bei einer festen Frequenz und Amplitude auf diese Störungen zu messen. Der Durchfluss der Streuteilchen durch das Messvolumen, und somit die zeitliche Abfolge der Messzeitpunkte ist statistisch verteilt. Die Zeitabhängigkeit der einzelenen Geschwindigkeitskomponenten soll an jedem Messpunkt in der Form

$$
u(t)=\bar{u}+\tilde{u}(t \bmod T)+u^{\prime}(t)
$$

aus den Messwerten rekonstriert werden, die in Gleichanteil $\bar{u}$, periodischen Anteil $\tilde{u}$ und turbulenten Anteil $u^{\prime}$ zerlegt werden. An diese Messwerte wird eine periodische Funktion mit vier Fourierkoeffizienten angepasst, die neben dem Gleichanteil und der Komponente bei der Grundfrequenz 1/T mit der Periodendauer der Anregung $T$ noch die drei höheren Harmonischen bei den Frequenzen 2/T, 3/T und 4/T enthält. Tastet man nun das periodische Signal $u(t)$ mit der Grundfrequenz $1 / T$ an einer Folge $\left\{t_{n}\right\}$ von $N$ Zeitpunkten ab, so lassen sich die gemessenen Werte $u\left(t_{n}\right)$ mittels dieses Modells darstellen als

$$
u\left(t_{n}\right)=\sum_{m=-4}^{4} S_{m} e^{i 2 \pi m \phi_{n}}+u^{\prime}\left(t_{n}\right) ; \quad \phi_{n}=t_{n} / T-\phi_{0}
$$

mit 9 komplexen spektralen Amplituden $S_{m}$, die bestimmt werden sollen. $\phi_{0}$ ist dabei die Bezugphase der Anregung (entweder Schwinghinterkante oder Membranen). In Matrixschreibweise wird die Gleichung (2.3) zu

$$
\underline{\mathrm{U}}=\underline{\underline{A}} \cdot \underline{\mathrm{S}}+\underline{\mathrm{U}}^{\prime}
$$

mit $\underline{\mathrm{U}}=\left(u\left(t_{1}\right), \ldots, u\left(t_{N}\right)\right)^{T}$ und $\underline{\mathrm{S}}=\left(S_{-4}, \ldots, S_{4}\right)^{T}$ sowie $\underline{\mathrm{U}}^{\prime}=\left(u^{\prime}\left(t_{1}\right), \ldots, u^{\prime}\left(t_{N}\right)^{T}\right)$ dargestellt. Hierbei ist die Matrix $\underline{\underline{A}}$ eine $N \times 9$-Matrix, die nur aus Phaseninformationen besteht $\left(\underline{\underline{A}}=\left(e^{i 2 \pi q \phi_{p}}\right)_{p q}, 1<\bar{p}<N,-4 \leq q \leq 4\right)$. Die spektrale Amplitude $S_{m}$ wird nun durch Anpassung an die Messwerte gemäß Gleichung (2.5) bestimmt (NEUwALD $[17])$.

$$
\underline{\mathrm{S}}=\underline{\underline{E^{-1}}} \underline{\underline{A^{T}}} \cdot \underline{\mathrm{U}} ; \quad \underline{\underline{E}}=\underline{\underline{A^{T}}} \cdot \underline{\underline{A}}, \quad \underline{\underline{A^{T}}} \cdot \underline{\mathrm{U}^{\prime}}=0
$$


Bei äquidistant abgetasteten Signalen erhält man die erwünschte Information über das Signalspektrum durch diskrete Fouriertransformation der phasengemittelten Messwerte, wobei die Matrix $\underline{\underline{E}}$ einer Einheitsmatrix entspricht. Diese liefert allerdings bei der zufälliger Abtastung aufgrund der stochastischen Verteilung der Streuteilchen nur einen Schätzwert der gesuchten spektralen Amplitude. Aus diesem Grund ist eine Realisation bei der Messung dann beendet, wenn genügend Messwerte gesammelt sind und diese hinreichend über die Periode verteilt sind, d.h. wenn die Determinante von $\underline{\underline{E}}$ groß genug ist. Andernfalls, wenn sie unter den Wert 0.1 fällt, wird die Anzahl der Abtastwerte zunächst weiter erhöht.

Es werden zunächst zehn solcher Realisationen durchgeführt, und dann wird die Unsicherheit der Messwert abgeschätzt. Diese Unsicherheit der Messwerte ergibt sich aufgrund turbulenter Schwankungen in der Strömung, sowie durch die oben beschriebenen Fehler des LDA, und schließlich durch die Diskretisierung der Zeitinformation $(\Delta t=T / 256)$. Für die Unsicherheit einer Frequenzkomponente $\Delta S_{m}$ wird die dreifache Varianz des Mittelwertes $S_{m}$ angenommen:

$$
\Delta S_{m}=3 \sqrt{\frac{\operatorname{var}\left(S_{m}\right)}{K}} ; \quad \operatorname{var}\left(S_{m}\right)=\frac{1}{K-1} \sum_{k=1}^{K}\left(S_{m}^{(k)}-S_{m}\right)^{2}
$$

Dabei ist $\operatorname{var}\left(S_{m}\right)$ die Varianz der Ergebnisse bei $K$ Realisationen. Mit zunehmender Anzahl der Realisationen konvergiert die Varianz gegen einen endlichen Wert, während der Fehler des Mittelwertes gegen Null geht.

Als Maß für die turbulenten Schwankungen in der Strömung dient das zeitliche Mittel über die inkohärenten Schwankungen.

$$
\overline{\left(u^{\prime}(t)\right)^{2}}=\overline{(u(t)-\{\bar{u}+\tilde{u}(t \bmod T)\})^{2}}
$$

Dazu wird zu jedem einzelnen Phasenwert für alle Abtastwerte der kohärente Anteil des Signals

$$
\langle u\rangle_{\phi_{i}}:=\bar{u}+\tilde{u}\left(\phi_{i}\right)
$$

rekonstruiert, quadriert, von der Quadratsumme der ursprünglichen $N_{i}$ Geschwindigkeitsmesswerte $u_{j}\left(\phi_{i}\right)$ abgezogen und anschließend über alle Abtast- bzw. alle 256 Phasenwerte gemittelt. Aus dieser Abschätzung wird nun eine Effektivgeschwindigkeit für die auftretenden Fluktuationen bestimmt:

$$
\left(u_{e f f}^{\prime}\right)^{2}=\frac{1}{256} \sum_{i=0}^{255}\left(\frac{1}{N_{i}}\left[\sum_{j=1}^{N_{i}}\left[u_{j}\left(\phi_{i}\right)\right]^{2}\right]-\left[\langle u\rangle_{\phi_{i}}\right]^{2}\right)
$$

\subsubsection{Ablauf der Messung}

Vor Beginn der Messung muss die Justierung der optischen Komponenten des LDAs durchgeführt werden. Bei dieser Einstellung soll man darauf achten, dass die parallelen Laserstrahlen relativ zur Splitterplattenoberfläche exakt einen Winkel von $0^{\circ}$ (für die 
$u$-Geschwindigkeitskomponente) bzw. $90^{\circ}$ (für die $v$-Komponente) erhalten. Die Fokussieroptik des Photomultipliers wird ebenfalls vor jeder Messung sorgfältig justiert, da die Datenrate davon abhängt.

Das gesamte Wasser, das sich in dem $350 l$ fassenden Kunststofftank befindet, wird vor dem Betrieb in einem Vakumbehälter entgast, so dass sich während der Messung keine Luftblasen an den Kanalwänden oder den Sieben absetzen. Dann wird das Wasser mit einer Hilfspumpe von unten in den Kanal gedrückt und dabei darauf geachtet, dass keine Luftblasen unter den Sieben entstehen. Die Steiggeschwindigkeit kann mit der Pumpe sehr gut eingestellt werden. Ist das Wasser auf das Niveau des Beruhigungsbeckens gestiegen, wird die eigentliche Pumpe angestellt und dann der Verschluss im Kunststofftank geöffnet. Anschließend wird der Schieber eingestellt, bis die gewünschte Strömungsgeschwindigkeit erreicht ist.

Während des Betriebs wird die Drehzahl der Pumpe so geregelt, dass möglichst wenig Wasser in die Überlaufrohre fließt, da das über Überlaufrohre direkt in den Kunststofftank zurückgeleitete Wasser sonst Vibrationen dieser Rohre hervorruft, die sich auf den Kanal übertragen könnten. Außerdem wird dadurch die kinetische Energie von Querströmungen im Beruhigungsbecken so gering wie möglich gehalten.

Bevor die eigentliche Messung startet, müssen einzelne Profilmessungen von $\bar{u}$ in der Grenzeschicht stromauf der Plattenhinterkante in unmittelbarer Nähe der Platte als Funktion von $y$ durchgeführt werden, da diese Messungen zu einer sehr genauen Eichung des Nullpunktes der $y$-Achse, d.h. der Plattenoberfläche, ausgenutzt werden können.

Das in Abschnitt 2.3.2 beschriebene Verfahren ist inklusive Ansteuerung des Verfahrtisches und der Anregungsvorrichtungen in einem PC-Computerprogramm zusammengefasst. Zuerst wird eine Datei mit dem Messgitter erstellt. Sobald die Messung bei dem erstem Punkt des Messgitters startet, wird nach 180 Abtastwerten überprüft, ob mindestens 36 verschiedene Phasenwerte besetzt sind. Ist das nicht der Fall, wird die Anzahl der Samples um den Faktor 1.5 erhöht und weiter gemessen. Anschließend werden 5 Frequenzenanteile bzw. der Effektivwert der turbulenten Schwankungen des Signals nach dem oben beschriebenen Verfahren berechnet und auf den Bildschirm gezeigt.

Nach mindestens 10 solcher Realisationen werden die Mittelwerte und Varianzen der 5 Spektralanteile berechnet. Ist der relative Fehler in der Spektralkomponente, die zur Anregungsfrequenz gehört, kleiner als 10\% oder ist das Auflösungsvermögen des LDAs erreicht, wird die Messung bei dem Messgitter-Punkt beendet, und die Ergebnisse werden in einer Datei abgespeichert. Andernfalls werden weitere Realisationen durchgeführt. Anschließend wird der nächste Messpunkt des Gitters angefahren. Die Messung läuft nach Start des Programms vollautomatisch ab. Eventuelle Messfehler oder Unregelmäßigkeiten werden in einem Messprotokoll erfasst.

Die Vermessung einer Komponente des Geschwindigkeitsfeldes für eine Anregungsart dauert je nach Datenrate am Counter etwa 20-24 Stunden für etwa 600 Messpunkte. Die vollständige Vermessung der Felder für eine Frequenz, d.h. $u$ - und $v$-Komponente bei gleichzeitiger Anregung durch den Druckgradienten und die Schwingkante sowie $u$ - und $v$-Komponente bei Anregung nur durch den Druckgradiente bzw. nur die Schwingkante, 
dauert somit etwa 6 Tage. Die Verschiebung des Messvolumens durch die Schrittmotoren stellt dabei einen wesentlichen Zeitfaktor dar. 


\section{Kapitel 3}

\section{Die Gleichströmung}

In diesem Kapitel werden Messergebnisse für den stationären Anteil der Kanalströmung dargestellt, der sich aus der Gleichkomponente des Fourierspektrums bei der phasenrichtigen Mittelung (siehe Kapitel 2) ergibt. Die Gleichströmung kann daher gleichzeitig mit den Messdaten der Wechselgeschwindigkeit erfasst werden. Nach einem Umbau des Kanals durch EnghardT [9] ist die Strömung des Kanals außerhalb der Seitenwandgrenzschichten weitgehend zweidimensional.

\subsection{Die $\bar{u}$-Komponente}

Abbildung 3.1 zeigt die $\bar{u}$-Komponente als Funktion von $x$ und $y$. Der Betrachter blickt in die Hauptströmungsrichtung, also in $x$-Richtung. Deutlich zu erkennen ist der Übergang vom Grenzschichtprofil zum tanh-ähnlichen Profil der freien Scherschicht sowie deren allmähliche Aufweitung. Man sieht, dass die Grenzschichtdicke im Hauptströmungsbereich an der Hinterkante ca. $10 \mathrm{~mm}$ erreicht hat, nachdem das Wasser entlang der ca. $900 \mathrm{~mm}$ langen Splitterplatte geströmt ist. Dort steigt die Strömungsgeschwindigkeit also von Null an der Plattenoberfläche auf einen im Außenbereich der Grenzschicht konstanten Wert von $U_{1} \approx 15 \mathrm{~cm} / \mathrm{s}$. Bemerkenswert ist die zeitliche Konstanz von $U_{1}$, wenn mann bedenkt, dass zwischen der Messung des ersten Punktes bei $x=30 \mathrm{~mm}$ und des letzten bei $x=-5 \mathrm{~mm}$ etwa zwölf Stunden lagen.

Die Plattengrenzschichten der beiden Seiten der Platte treffen an der Hinterkante aufeinander und gehen in eine freie Scherschicht über. Eine Nachlaufdelle entsteht durch diese Zusammenführung der beiden Grenzschichten. Sie füllt sich aufgrund der dicken Grenzschicht der Nebenströmungsseite mit wachsendem Abstand von der Plattenhinterkante nur langsam auf, wobei das Geschwindigkeitsminimum zu negativen $y$-Werten wandert. Schließlich bildet sich ein weitgehend symmetrisches Scherschichtprofil aus, das durch eine tanh-Funktion genähert werden kann. Ein Profil mit einem Wendepunkt ist besonders empfindlich gegen Störungen, so dass sich dort durch instationäre Anregungen der Strömung eine Kelvin-Helmholtz-Instabilität ausbilden kann. 


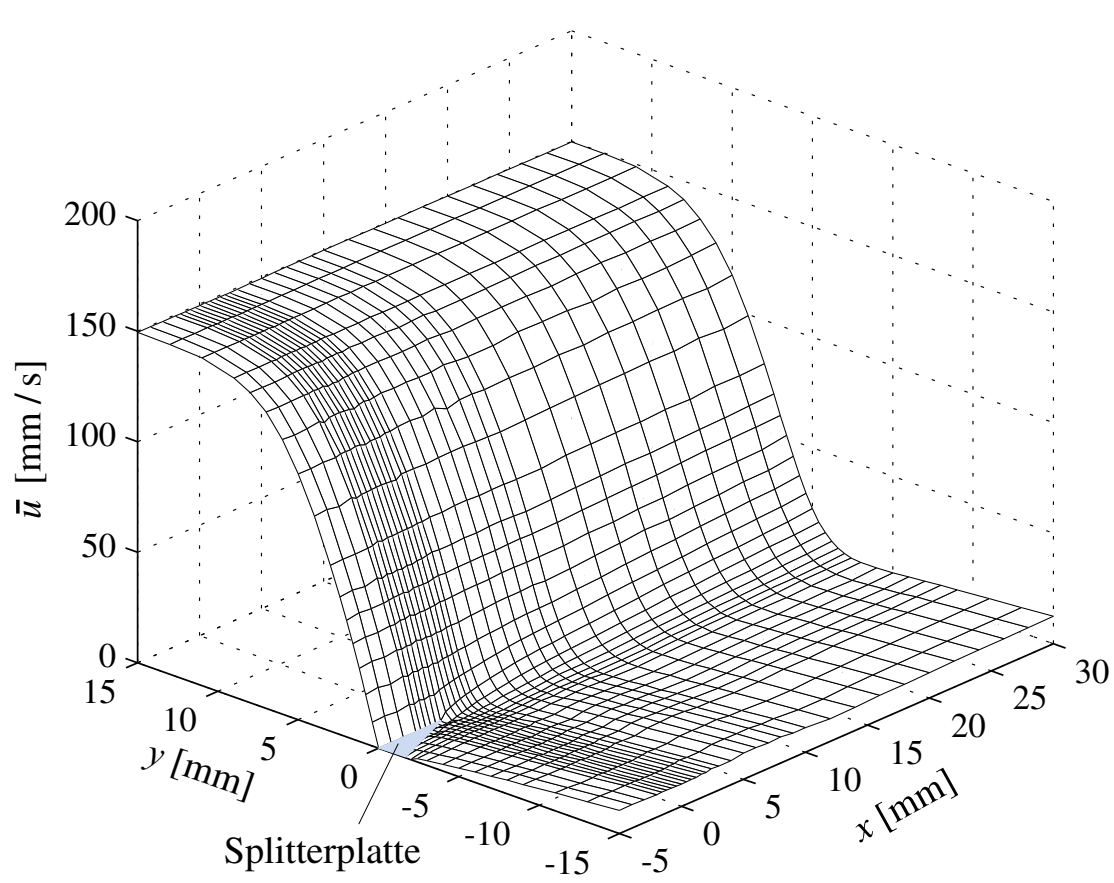

Abb. 3.1: Die Gleichgeschwindigkeitskomponente $\bar{u}$ in der Plattengrenzschicht und der Übergang in eine Scherschicht stromab der Hinterkante der Splitterplatte

\subsubsection{Das Grenzschicht- und das Scherschichtprofil}

Abbildung 3.2 (a) zeigt ein gemessenes Profil der Strömungsgeschwindigkeit in der Wandgrenzschicht als Funktion von $y$ bei $x=-30 \mathrm{~mm}$, also $30 \mathrm{~mm}$ stromauf der Hinterkante der Splitterplatte und das daran mittels der zwei freien Parameter angepasste Blasius-Profil mit einer Pohlhausen-Korrektur nach Schlichting [24]. In Abbildung 3.2 (b) sind die zu den Profilmesswerten gehörenden turbulenten Fluktuationen $u_{\text {eff }}^{\prime}$ und Messfehler $\Delta \bar{u}$ dargestellt. Das angepasste theoretische Profil stimmt sehr gut mit der Messwerten überein; der Anpassungsfehler (grüne Kurve) liegt nahezu innerhalb der Messfehler, obwohl eine systematische Struktur dabei erkennbar ist. Diese systematische Abweichung könnte daran liegen, dass die Pohlhausen-Korrektur aus einen Polynom vierten Grades besteht. Die Messfehler liegen im Potenzialbereich der Strömung unter $0.03 \%$ der Hauptströmungsgeschwindigkeit $U_{1}$ und damit unterhalb der Auflösungsgrenze des LDAs, nehmen in der Grenzschicht aufgrund der endlichen Breite des Messvolumens jedoch auf etwa $0.3 \%$ zu. Die Effektivwerte $u_{\text {eff }}^{\prime}$ betragen im Potenzialbereich $0.7 \%$, und in der Grenzschicht etwa $1.0 \%$ von $U_{1}$.

Das typische Profil der Scherschicht, das bei $x=30 \mathrm{~mm}$ aufgenommen wurde, wird in Abbildung 3.3 dargestellt. Diese Messwerte können dabei, wie EnGHardT [9] gezeigt hat, über die 11 angepassten Parameter $u_{0}, u_{1}, m, \Theta_{f}, y_{0}, y_{1}, y_{2}, a, b, c$ und $d$ durch eine differenzierbare Funktion 

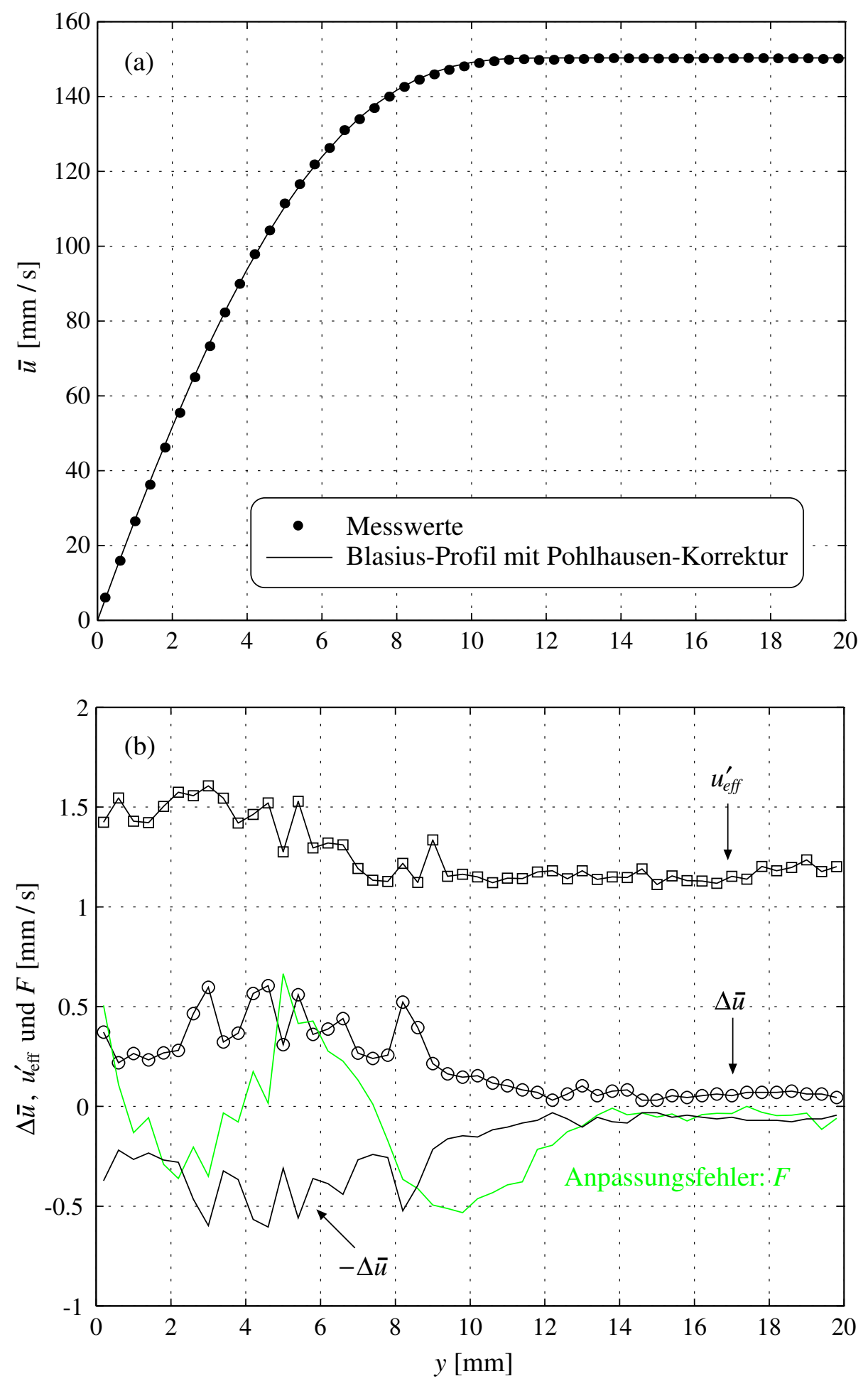

Abb. 3.2: (a) Das Grenzschichtprofil bei $x=-30 \mathrm{~mm}$, mit angepasstem Blasiusprofil mit Pohlhausen-Korrektur und (b) Effektivwerte der turbulenten Fluktuationen $u_{\text {eff }}^{\prime}(y)$, die Unsicherheit der Messwerte $\Delta \bar{u}(y)$ und der Anpassungsfehler $F$ als Funktion von $y$. 

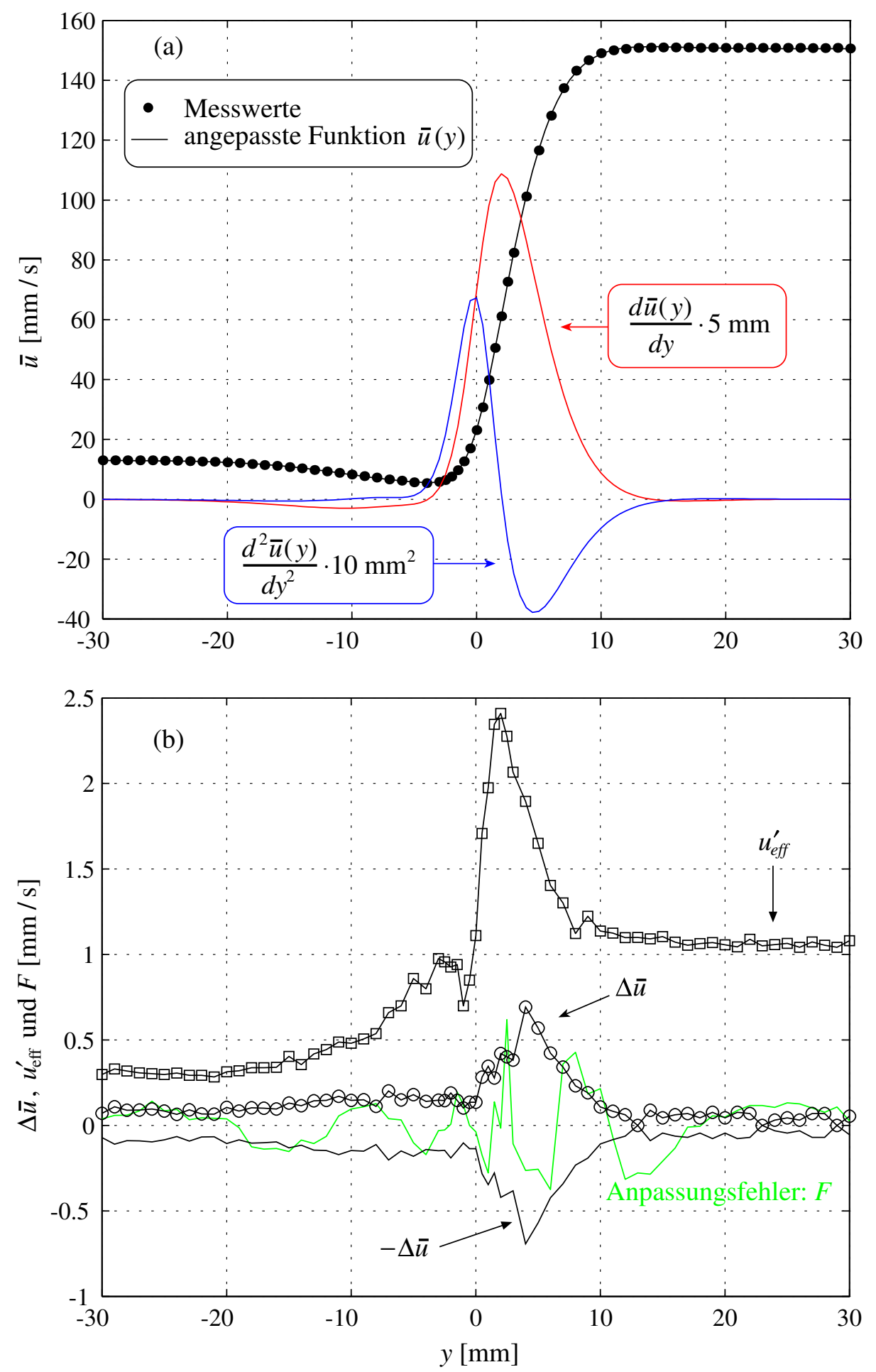

Abb. 3.3: (a) Das Scherschichtprofil bei $x=30 \mathrm{~mm}$ mit der angepassten Funktion $\bar{u}(y)$ sowie deren 1. und 2. Ableitung, (b) Effektivwerte der Fluktuationen $u_{\text {eff }}^{\prime}(y)$, die Unsicherheit der Messwerte $\Delta \bar{u}(y)$ und der Anpassungsfehler $F$ als Funktion von $y$. 


$$
\bar{u}(y)=u_{0}-u_{0}\left(1+m e^{\frac{m}{\Theta_{f}}\left(y-y_{0}\right)}\right)^{-\frac{1}{m}}+u_{1}+\frac{a\left(y-y_{1}\right)+b\left(y-y_{1}\right)^{2}+c\left(y-y_{1}\right)^{3}}{\cosh ^{2}\left(d\left(y-y_{2}\right)\right)}
$$

beschrieben werden, so dass auch die beiden ersten Ableitungen von $\bar{u}(y)$ analytisch angegeben werden können. Diese Funktion geht in eine tanh-Funktion über, wenn man $y_{0}=y_{1}=y_{2}=a=b=c=d=0$ und $m=1$ setzt:

$$
\bar{u}(y)=u_{0}-u_{0}\left(1+e^{\frac{y}{\Theta_{f}}}\right)^{-1}=\frac{u_{0}}{2}\left(1+\tanh \left(\frac{y}{2 \Theta_{f}}\right)\right)
$$

Sowohl die an die Messwerte angepasste Funktion als auch die - aus Übersichtlichkeitsgründen - skalierte erste und zweite Ableitung von $\bar{u}(y)$ werden nun in Abbildung 3.3 (a) gezeigt. In Abbildung 3.3 (b) sind wiederum die turbulenten Fluktuationen $u_{\text {eff }}^{\prime}$ und die Messfehler $\Delta \bar{u}$ dargestellt, die miteinander sehr gut korreliert sind. Es ist deutlich zu erkennen, dass die angepasste Funktion die Messwerte gut wiedergibt; der Anpassungsfehler (grüne Kurve) liegt nahezu unsystematisch innerhalb des Messfehlers. Die Messfehler im Potenzialbereich haben etwa gleiche Werte wie bei der Messung stromauf der Hinterkante, nehmen allerdings in der Scherschicht auf etwa $0.5 \%$ von $U_{1}$ zu. Die turbulenten Schwankungen sind dabei auch im Bereich der Scherschicht aufgrund des endlichen Messvolumens größer als im Potenzialbereich. Man erkennt deshalb eine gewisse Ähnlichkeit zwischen den Profilen von $u_{\text {eff }}^{\prime}(y)$ und von $d \bar{u}(y) / d y$. Diese Effektivwerte erreichen bis zu $1.7 \%$ von $U_{1}$.

\subsubsection{Strömungsparameter}

Zur Charakterisierung der Profile werden üblicherweise die Impulsverlustdicke $\Theta$, die Verdrängungsdicke $\delta_{1}$ und der Formfaktor $H_{12}$ verwendet, die folgendenmaßen definiert sind:

$$
\begin{gathered}
\Theta:=\int_{-\infty}^{\infty} \frac{\bar{u}(y)-U_{2}}{U_{1}-U_{2}}\left(1-\frac{\bar{u}(y)-U_{2}}{U_{1}-U_{2}}\right) d y \\
\delta_{1}:=\int_{0}^{\infty}\left(1-\frac{\bar{u}(y)}{U_{1}}\right) d y, \quad H_{12}:=\frac{\delta_{1}}{\Theta}
\end{gathered}
$$

Die angegebene Definition der Grenzschichtdicken gilt allgemein für Scherschichten, wenn $\bar{u}(y)$ eine monotone Funktion ist; $\delta_{1}$ und $H_{12}$ können nur für wandgebundene Scherschichten definiert werden. In freien Scherschichten bezeichnen $U_{1}$ und $U_{2}$ die Geschwindigkeiten in der Haupt- und in der Nebenströmung im großen Abstand von der Scherschicht. Da hier $\bar{u}(y)$ nicht monoton ist, wird $U_{2}$ gleich dem Minimum von $\bar{u}(y)$ gesetzt und von dieser Position ab integriert.

Ein weiterer Parameter der Strömung ist die mit der Impulsverlustdicke gebildete Reynoldszahl $R_{\Theta}=U_{1} \Theta / \nu$. Sie ist eine Kennzahl für die Zähigkeitseffekte der Strömung. Die genannten Größen sind in Tabelle 3.1 zusammengestellt. 


\begin{tabular}{|c||c|c|c|c|}
\hline$U_{1}$ & $\Theta[\mathrm{mm}]$ & $\delta_{1}[\mathrm{~mm}]$ & $H_{12}$ & $\operatorname{Re}_{\Theta}$ \\
\hline \hline $15 \mathrm{~cm} / \mathrm{s}$ & 1.42 & 3.32 & 2.34 & 213 \\
\hline
\end{tabular}

Tabelle 3.1: Die Kennzahlen der Kanalströmung bei $x=-30 \mathrm{~mm}$

Zum Vergleich beträgt der Formfaktor für die zweidimensionale Blasiusgrenzschicht $H_{12}=2.59$. Für die Abweichung des Formfaktors im Vergleich zum Blasiusprofil der ebenen Plattengrenzschicht ist ein kleiner negativer Druckgradient verantwortlich. Dieser Druckgradient entsteht dadurch, dass die Grenzschichten an den Kanalwänden mit zunehmendem Laufweg anwachsen und aufgrund der Verdrängungswirkung die Strömung beschleunigen.

\subsubsection{Inhomogenität des $\bar{u}$-Profils}

Wie man in Abbildung 3.1 sieht, entwickelt sich das Scherschichtprofil mit wachsendem Abstand von der Ablösekante. Der Gradient des Profils $\partial \bar{u} / \partial y$ ist nahe der Hinterkante sehr steil, wird jedoch mit steigender $x$-Koordinate flacher. Für diese Entwicklung ist die Diffusion der Gleichwirbeligkeit verantwortlich. Bei der dreidimensionalen Darstellung von $\partial \bar{u} / \partial x$ in Abbildung 3.4 ist zu erkennen, dass sich starke Änderungen des $\bar{u}$-Profils in $x$-Richtung besonders im Bereich nahe der Hinterkante ergeben, während sie im Bereich der Potenzialströmung und weit stromab der Hinterkante nur klein sind. Betrachtet man das in Abbildung 3.5 (a) dargestellte $\bar{u}$-Profil als Funktion von $x$ nur bei $y=0$, so erkennt man, dass der Gradient $\partial \bar{u} / \partial x$ in unmittelbarer Nähe der Hinterkante sehr groß sein muss, da das Profil einen fast wurzelförmigen Verlauf zeigt. Die Abhängigkeit der Impulsverlustdicke $\Theta$ von der $x$-Koordinate zeigt einen ähnlichen Verlauf (siehe Abbildung 3.5 (b)). Man stellt somit fest, dass die Strömung im Bereich hinter der Ablösekante keineswegs als homogen in $x$-Richtung angesehen werden kann.

\subsection{Die $\bar{v}$-Komponente}

Abbildung 3.6 zeigt die $\bar{v}$-Komponente als Funktion von $x$ und $y$. Sie ist um zwei Größordnungen kleiner als die $\bar{u}$-Komponente und nimmt maximale Werte stromab der Hinterkante im Bereich der Nebenströmung an. Stromauf der Hinterkante auf der Hauptströmungsseite $(x<0, y>0)$ ist $\bar{v}$ negativ. Man erwartet zunächst ein umgekehrtes Vorzeichen, da das Medium aufgrund der Plattengrenzschicht, die mit der Lauflänge entlang der Splitterplatte anwächst, verdrängt wird, also $\bar{v}$ hier positiv sein soll. Aus demselben Grund erwartet man auf der Nebenströmungsseite $(x<0, y<0)$ ein negatives $\bar{v}$.

Da aber an der Hinterkante, wo die Hauptströmung und Nebeströmung zusammen laufen, ein Unterdruck entsteht und die Hauptströmung den Unterdruck im Bereich $-5 \mathrm{~mm}<x<0 \mathrm{~mm}$ schon spürt, ist $\bar{v}$ dort negativ. Auf der Nebenströmungsseite ist $\bar{v}$ nicht nur aus diesem Grund positiv, sondern auch deswegen, weil die Platte an dieser 


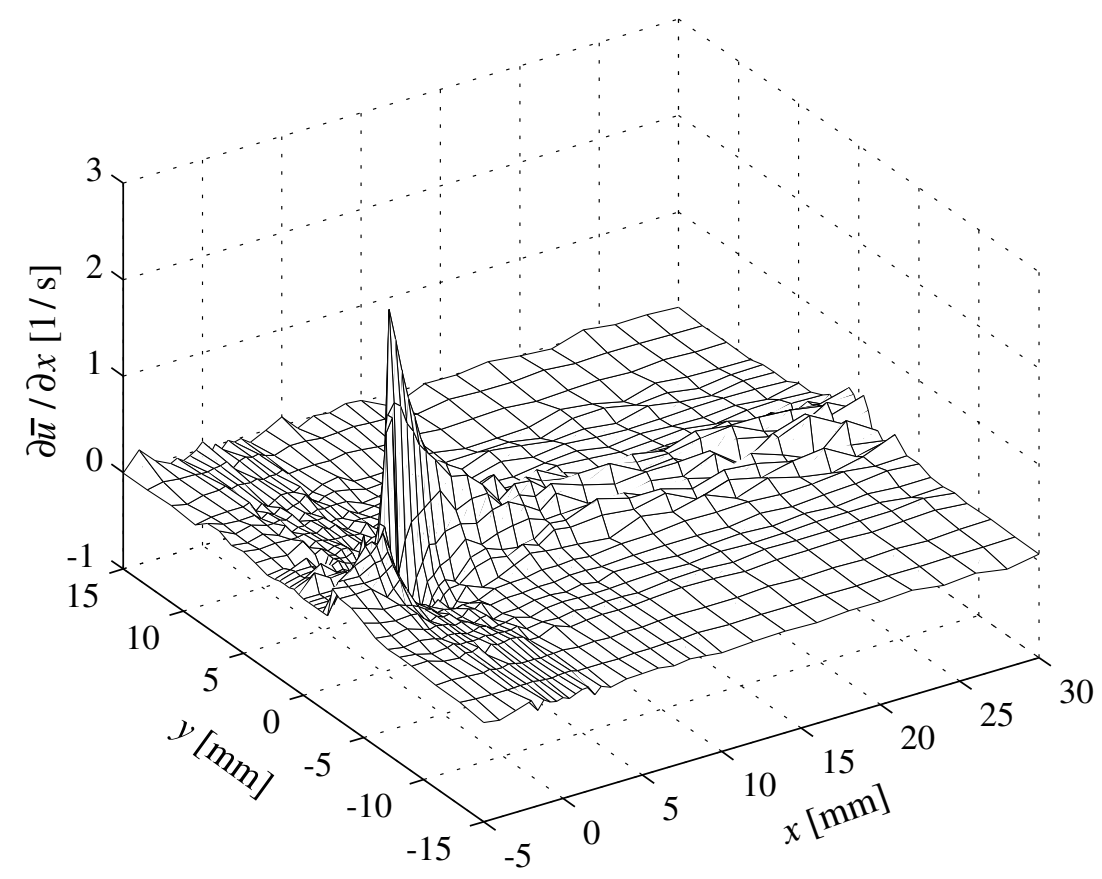

Abb. 3.4: Die Änderung des $\bar{u}$-Profils in $x$-Richtung: $\partial \bar{u} / \partial x$
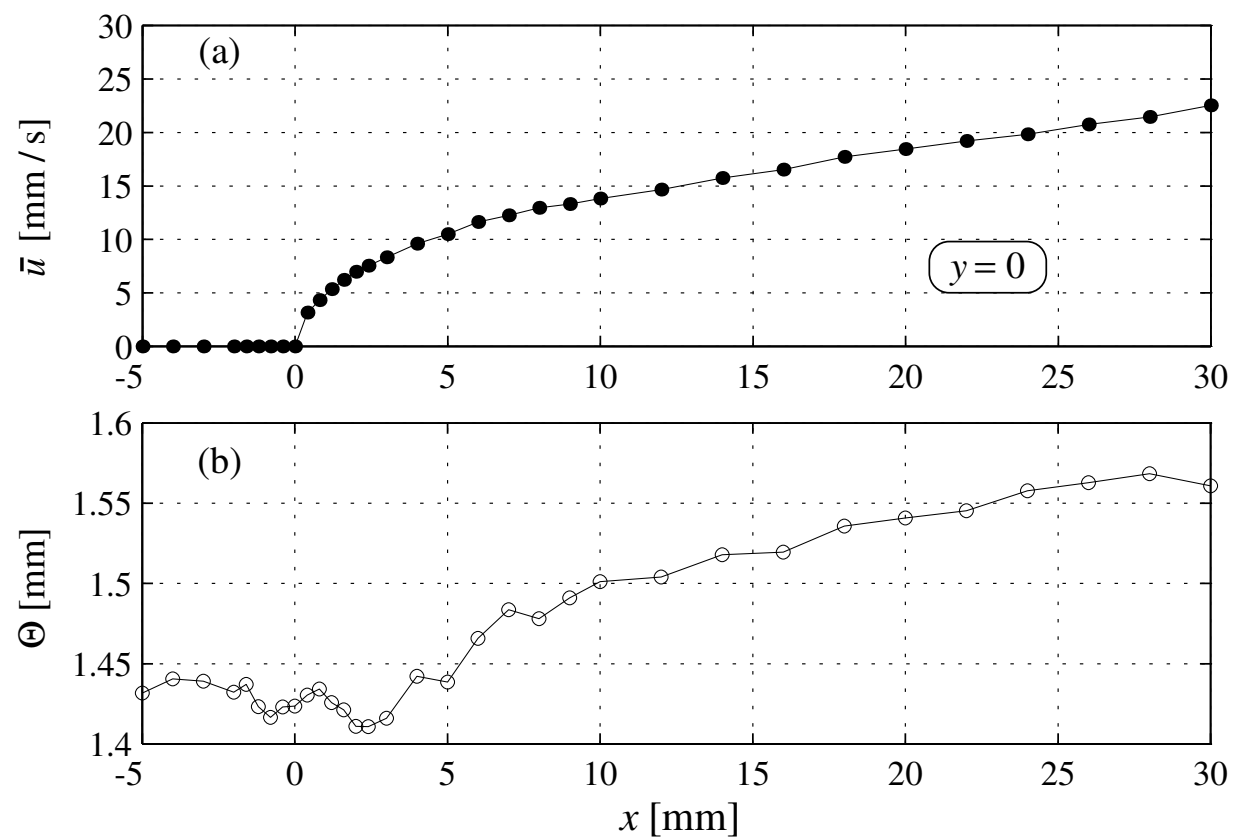

Abb. 3.5: (a) $\bar{u}$ bei $y=0$ als Funktion von $x$ und (b) die Impulsverlustdicke $\Theta$ als Funktion von $x$ 


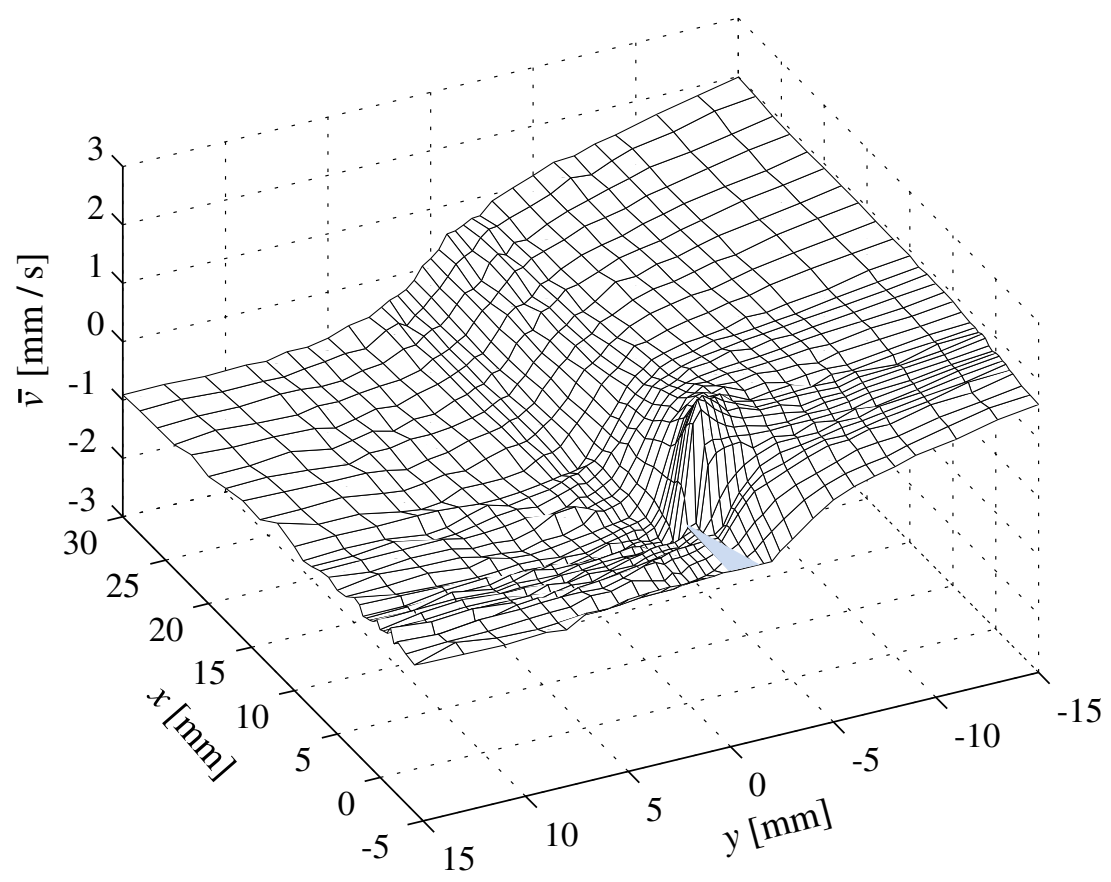

Abb. 3.6: Die Gleichgeschwindigkeitskomponente $\bar{v}$ in der Plattengrenzschicht und der Übergang in eine Scherschicht stromab der Hinterkante der Splitterplatte

Seite zur Hinterkante hin spitz zusammenläuft.

Stromab der Hinterkante $(x>0)$ ist $\bar{v}$ auf der Hauptströmungsseite negativ und auf der Nebenströmungsseite positiv. Die Strömung bewegt sich also in die Scherschicht hinein. Dadurch füllt sich eine Nachlaufdelle, sie sich im Bereich der Plattenhinterkante ausbildet, weiter stromab nach und nach auf. Wie man aus Abbildung 3.6 sieht $(\bar{v}$ ist für $y<0$ besonders groß), kommt das Medium, das diese Delle glättet, im wesentlichen aus der Nebenströmung. Die Hauptströmung ist relativ unbeeinlußt vom Ende der Platte, d. h. das Medium strömt praktisch geradeaus weiter.

Messtechnisch bedingt muss die Empfangsoptik für die Messung der $\bar{v}$-Komponente in der Hauptströmung etwas verdreht werden. Dadurch wird ein Teil der sehr viel größeren $\bar{u}$ - auf die $\bar{v}$-Komponente projiziert, was zu einer relativ großen Messunsicherheit führt. Der Justierfehler des Winkels der Schnittebene der Laserstrahlen liegt bei ca. $0.3^{\circ}$, womit die Unsicherheit der Geschwindigkeitsmessung der $\bar{v}$-Komponete mit max. $0.8 \mathrm{~mm} / \mathrm{s}$ abgeschätzt werden kann. Die obere Fehlergrenze liegt somit in der Größenordnung der gemessenen $\bar{v}$-Komponete. Im Bereich der Nebenströmung ist die Messunsicherheit von $\bar{v}$ durch diese Projektion etwa um eine Größenordnung kleiner.

\subsection{Die Gleichwirbeligkeit}

Die Aufweitung der freien Scherschicht aufgrund der Diffusion von Impuls quer zur Strömung wird besonders deutlich bei der Betrachtung der $z$-Komponente der stati- 


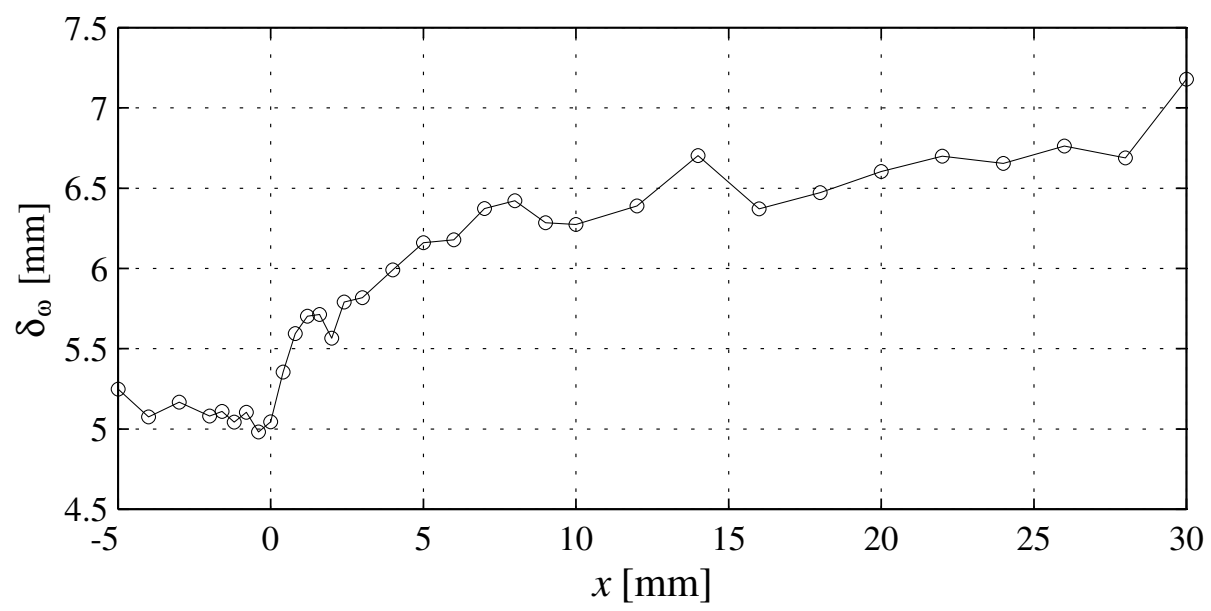

Abb. 3.7: Die Wirbeldicke $\delta_{\omega}$ als Funktion von $x$

onären Wirbeligkeit $\bar{\omega}$. Diese ist im wesentlichen durch den negativen wandnormalen Gradienten von $\bar{u}$ gegeben:

$$
\bar{\omega}=\frac{\partial \bar{v}}{\partial x}-\frac{\partial \bar{u}}{\partial y}
$$

da der erste Term $\partial \bar{v} / \partial x$ der Gleichung (3.5) vergleichsweise klein ist und nur im Bereich der Hinterkante, in der Nebenströmung, einen nennenswerten Beitrag liefert. Bei der dreidimensionalen Darstellung in Abbildung 3.8 (oben) blickt man in Strömungsrichtung, die Hauptströmung befindet sich auf der linken Seite. Bei der Betrachtung der Gleichwirbeligkeit werden besonders die Bereiche deutlich, in denen die Potenzialtheorie gültig ist, in denen also die Wirbeligkeit verschwindet. Die Illustration durch Konturlinien in Abbildung 3.8 (unten) zeigt, dass die Wirbeligkeitsverteilung an der Splitterplatte einen Sprung in $y$-Richtung hat, der kurz hinter der Kante sehr schnell durch Impulsdiffusion abflacht, und sich in den Nebenströmungsbereich hinein ausdehnt.

Neben der Impulsverlustdicke $\Theta$ ist auch die Wirbeldicke $\delta_{\omega}$ als Funktion von $x$ eine der charakteristischen Scherschichtgrößen. Sie ist definiert als:

$$
\delta_{\omega}:=\int_{-\infty}^{\infty} \frac{\bar{\omega}(y)}{\bar{\omega}_{\max }} d y,
$$

wobei $\bar{\omega}_{\max }=\max (\bar{\omega}(y))$. Auffällig ist in Abbildung 3.7, dass die Wirbeldicke hinter der Ablösekante, wie die Impulsverlustdicke $\Theta$, einen fast wurzelförmigen Verlauf zeigt, während sie sich vor der Hinterkante praktisch nicht mehr ändern. Aus der Auftragung erkennt man auch, dass sich eine starke Aufweitung der Scherschicht in unmittelbarer Nähe der Hinterkante ergibt; somit kann die Strömung in diesem Bereich nicht als homogen in $x$-Richtung angesehen werden. 

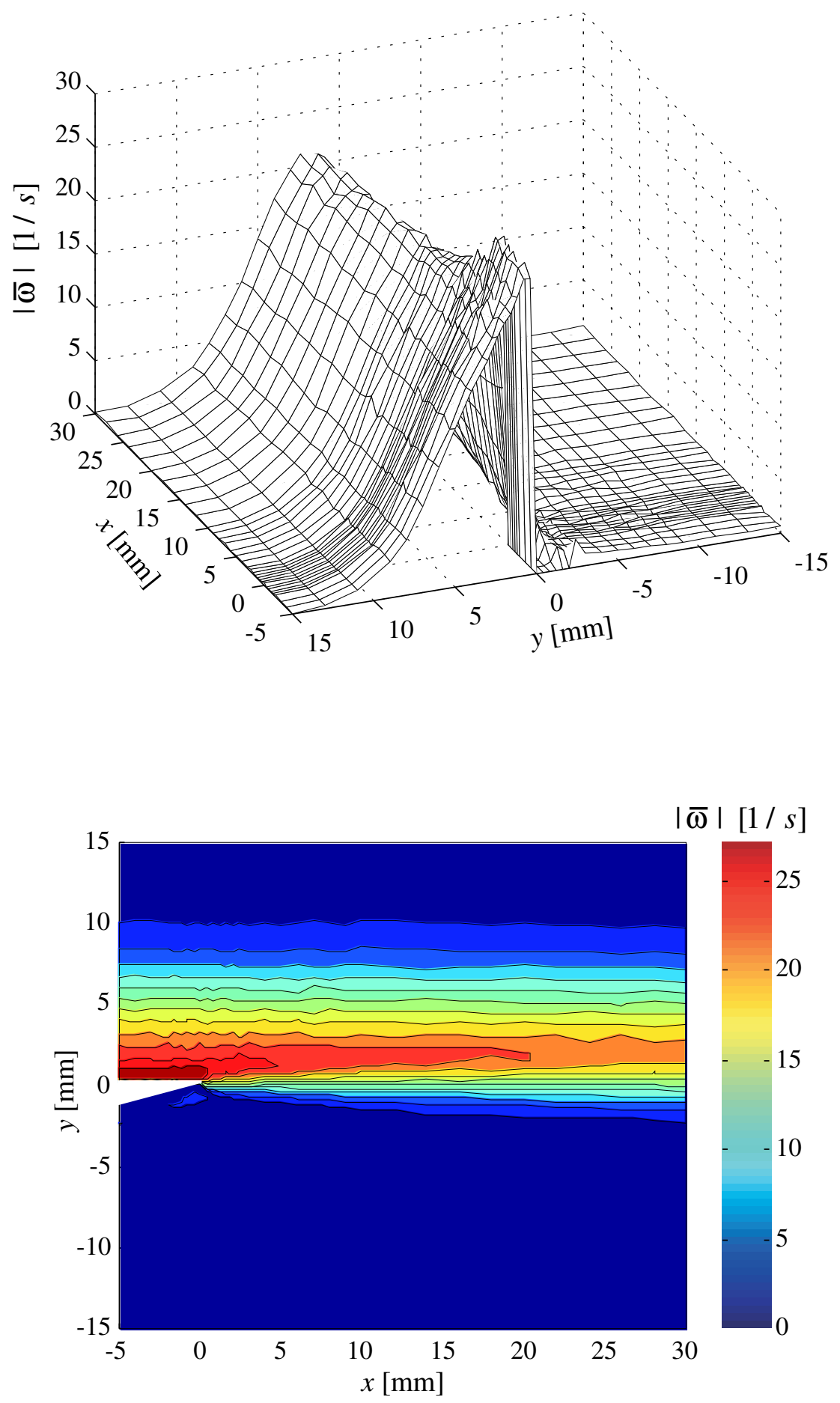

Abb. 3.8: Übersicht über die Gleichwirbeligkeit $\bar{\omega}$ : dreidimensionale Darstellung (oben) und Konturlinienbild (unten) 


\section{Kapitel 4}

\section{Anregbarkeit der Kelvin-Helmholtz-Instabilität}

Der genaue Mechanismus der Anregung der Kelvin-Helmholtz-Instabilität in einer freien Scherschicht durch eine zeitlich periodische Störung ist bis jetzt noch nicht bekannt. Ebenso ist keine physikalische Größe bekannt, die in unmittelbarer Nähe der Hinterkante bestimmt werden kann und die Amplitude und Phase der angeregten Instabilitätswelle festlegt. Zur Bestimmung einer solchen Größe sind Untersuchungen des Nahfeldes der Hinterkante notwendig, wo die Scherschicht empfindlich auf äußere Störungen reagiert. Es stellt sich nun die Frage, ob man ein solches Nahfeld experimentell bestimmen kann. Wenn diese Frage bejaht werden kann, führt dies zu der Frage, ob man eine maßgebende Größe von dem Nahfeld extrahieren kann, auf die man die Amplitude der erzeugten KH-Welle beziehen kann.

In diesem Kapitel werden zu diesem Zweck zuerst Eigenschaften der Instabilitätswelle dargestellt, danach werden Ergebnisse aus Messungen mittels der linearen Instabilitätstheorie analysiert und diskutiert.

\subsection{Die Abhängigkeit der Instabilitätswelle von der Strouhalzahl}

Ein wichtiger Parameter zur Beschreibung der KH-Welle ist die Strouhalzahl $S t_{\Theta}$, die mit der Impulsverlustdicke $\Theta_{0}$ bei $x=0$, der Hauptströmungsgeschwindigkeit $U_{1}$ und der Anregungsfrequenz $f$ gebildet ist: $S t_{\Theta}=2 \pi f \Theta_{0} / U_{1}$. Da in der vorliegenden Untersuchung die Parameter der Haupt- und der Nebenströmungsgeschwindigkeit $U_{1}$ und $U_{2}$ nicht variiert werden, ist $S t_{\Theta}$ proportional zur Anregungsfrequenz $f$.

Nimmt man an, dass die Strömung mit einer endlichen Dicke der Scherschicht in $x$ Richtung unendlich ausgedehnt und homogen ist, und ihr Profil durch ein tanh-Profil (siehe Gleichung (3.2)) beschrieben wird, kann sich eine Störung nach Michalke [15] nur im Bereich $0<S t_{\Theta}<0.25$ wellenartig ausbreiten. Da die in der Messstrecke vorhandenen Scherströmung, wie in Abbildung $3.4 \mathrm{zu}$ sehen ist, von der Hinterkante der Splitterplatte ablöst und von der $x$-Koordinate abhängt, ist eine exakte theoretische Beschreibung der KH-Welle nicht einfach. 


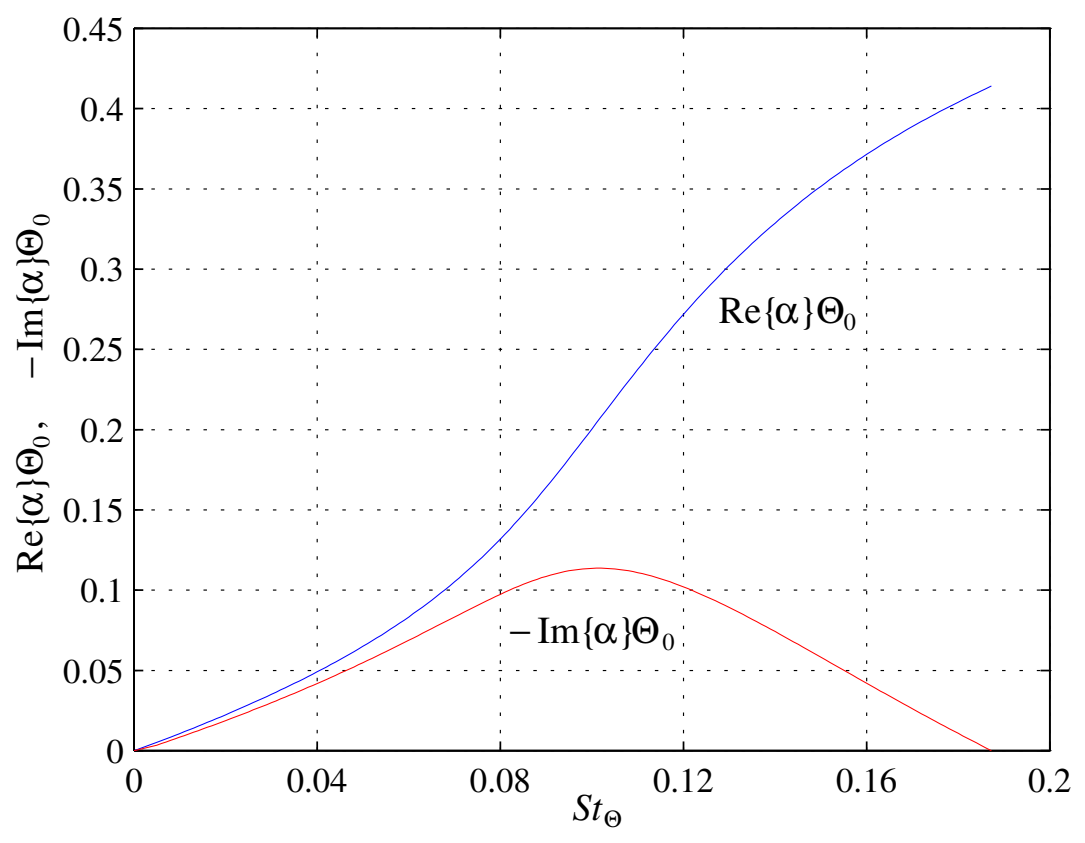

Abb. 4.1: Die Phasenkonstante $\operatorname{Re}\{\alpha\}$ und Anfachungskonstante $\operatorname{Im}\{\alpha\}$ der Instabilitätswelle in der Scherschicht bei $x=30 \mathrm{~mm}$, mit der Impulsverlustdicke $\Theta_{0}$ normiert

Trotz der Komplexität der vorliegenden Gleichströmung wird jedoch der Versuch unternommen, theoretische Instabilitätswellen zu berechnen, die die gemessenen Wechselgeschwindigkeiten beschreiben.

\subsubsection{Die Dispersionsbeziehung}

Zur theoretischen Beschreibung der Instabilitätswelle werden hier Gleichungen für die zweidimensionale, imkompressible Strömung (mit der Methode der kleinen Störungen) durch Zerlegung der Größen in einen stationären Anteil und einen Wechselanteil für die instationären Komponenten angegeben. Unter den weiteren Annahmen, dass

- die stationäre Strömung in $x$-Richtung, der Hauptströmungsrichtung, homogen ist und damit die Querkomponente $\bar{v}$ vernachlässigt werden kann,

- das Strömungsgebiet in $y$-Richtung unendlich ausgedehnt ist

- und der Einfluss der Zähigkeit von untergeordneter Bedeutung ist,

erhält man aus der Euler-Gleichung in linearer Näherung mit Hilfe der Stromfunktion die Rayleigh-Gleichung (Godreche \& Manneville [12]),

$$
\frac{d^{2} \psi_{0}(y)}{d y^{2}}=\left[\alpha^{2}+\frac{1}{\bar{u}(y)-c} \frac{d^{2} \bar{u}(y)}{d y^{2}}\right] \psi_{0}(y) \quad \text { mit } \quad c:=\frac{\beta}{\alpha} .
$$

Als Darstellung der Lösung wurde dabei 


$$
\tilde{\psi}(x, y, t)=\psi_{0}(y) \exp (i[\alpha x-\beta t]), \quad \tilde{u}(x, y, t)=\frac{\partial \tilde{\psi}}{\partial y} \quad \text { und } \quad \tilde{v}(x, y, t)=-\frac{\partial \tilde{\psi}}{\partial x}
$$

gewählt. Sowohl die Wellenzahl $\alpha$ als auch die Kreisfrequenz $\beta$ können komplex sein, was räumlich bzw. zeitlich angefachten oder gedämpften Lösungen entspricht. Da man sich hier für die räumliche Entwicklung der Schwingung interessiert, wird die Kreisfrequenz $\beta$ auf einen reellen Wert festgelegt und eine passende komplexe Wellenzahl $\alpha$ gesucht. Die Rayleigh-Gleichung mit den Randbedingungen $\psi_{0}(y) \rightarrow 0$ für $y \rightarrow \pm \infty$ stellt bei gegebenem Strömungsprofil $\bar{u}(y)$ eine Eigenwertgleichung dar, die numerisch gelöst werden muss. Das Profil $\bar{u}(y)$ wird aus den Messwerten gewonnen, in dem eine zweimal differenzierbare Funktion angepasst wird (Gleichung (3.1)). Die Dispersionsrelation $\alpha(\beta)$ ist nur von dem Gleichströmungsprofil $\bar{u}(y)$ abhängig.

In Abbildung 4.1 wird die mit der Impulsverlustdicke $\Theta_{0}$ normierte Wellenzahl über der Strouhalzahl $S t_{\Theta}$ dargestellt. Das Profil $\bar{u}(y)$ ist dabei aus Messwerten bei $x=$ $30 \mathrm{~mm}$ bestimmt. Da ein negativer Imaginärteil der Wellenzahl eine Anfachung der Störung bedeutet, wie in Abbildung $4.1 \mathrm{zu}$ sehen, ist die KH-Welle für $S t_{\Theta} \leq 0.19$ (hier Anregungsfrequenz $f \leq 2.9 \mathrm{~Hz}$ ) theoretisch anregbar. Der Realteil der Wellenzahl legt die Phasengeschwindigkeit bzw. die Phasen-Laufzeit der Instabilitätswelle fest.

Betrachtet man eine unendliche dünne Scherschicht (d.h. verschwindene Strouhalzahl $\left.S t_{\Theta}=0\right)$, so erhält man die analytische Dispersionsbeziehung

$$
\alpha=\frac{\beta}{\bar{U}\left(1+R^{2}\right)}(1 \mp i R), \quad \text { wobei } \quad \bar{U}=\frac{U_{1}+U_{2}}{2}, \quad R=\frac{U_{1}-U_{2}}{U_{1}+U_{2}}
$$

Hierbei ist $U_{1}$ die Geschwindigkeit oberhalb der Scherschicht und $U_{2}$ die unterhalb der Scherschicht (Godreche \& Manneville [12]). Bei dieser Dispersionsbeziehung ist $|\operatorname{Re}\{\alpha\}|=|\operatorname{Im}\{\alpha\}|$, wenn $U_{2} \ll U_{1}$ angenommen wird. Für die Scherschicht, im Experiment, mit endlicher Dicke, nähern sich daher die Phasen- und die Anfachungskonstante mit abnehmender Strouhalzahl einander an.

\subsubsection{Die Abhängigkeit der $\tilde{v}$-Komponente von der Strouhal- zahl}

Die gemessenen Wechselgeschwindigkeiten $\tilde{u}$ und $\tilde{v}$, die durch künstliche Störungen bei Überlagerung einer Gleichströmung angeregt werden, können die Rezeptivität einer freien Scherschicht experimentell charakterisieren. Das gemessene Wechselströmungsfeld ist allerdings sehr komplex, da das Feld nicht nur durch die KH-Welle bestimmt wird, sondern auch durch die Ablösungsbedingung an der Hinterkante, die von der Anregungsfrequenz bzw. der Strouhalzahl abhängt. Die angeregte KH-Welle ist besonders außerhalb des Nahfeldes zu sehen, da die angefachte Mode mit steigender $x$-Koordinate exponentiell anwächst.

Sowohl für die Untersuchung der Rezeptivität der Scherschicht, als auch der Ablösungsbedingungen, ist die $\tilde{v}$-Komponente zu beachten, da die Auslenkung der Scherschicht $\tilde{\eta}$ den folgenden engen Zusammenhang mit der $\tilde{v}$-Komponente hat: 

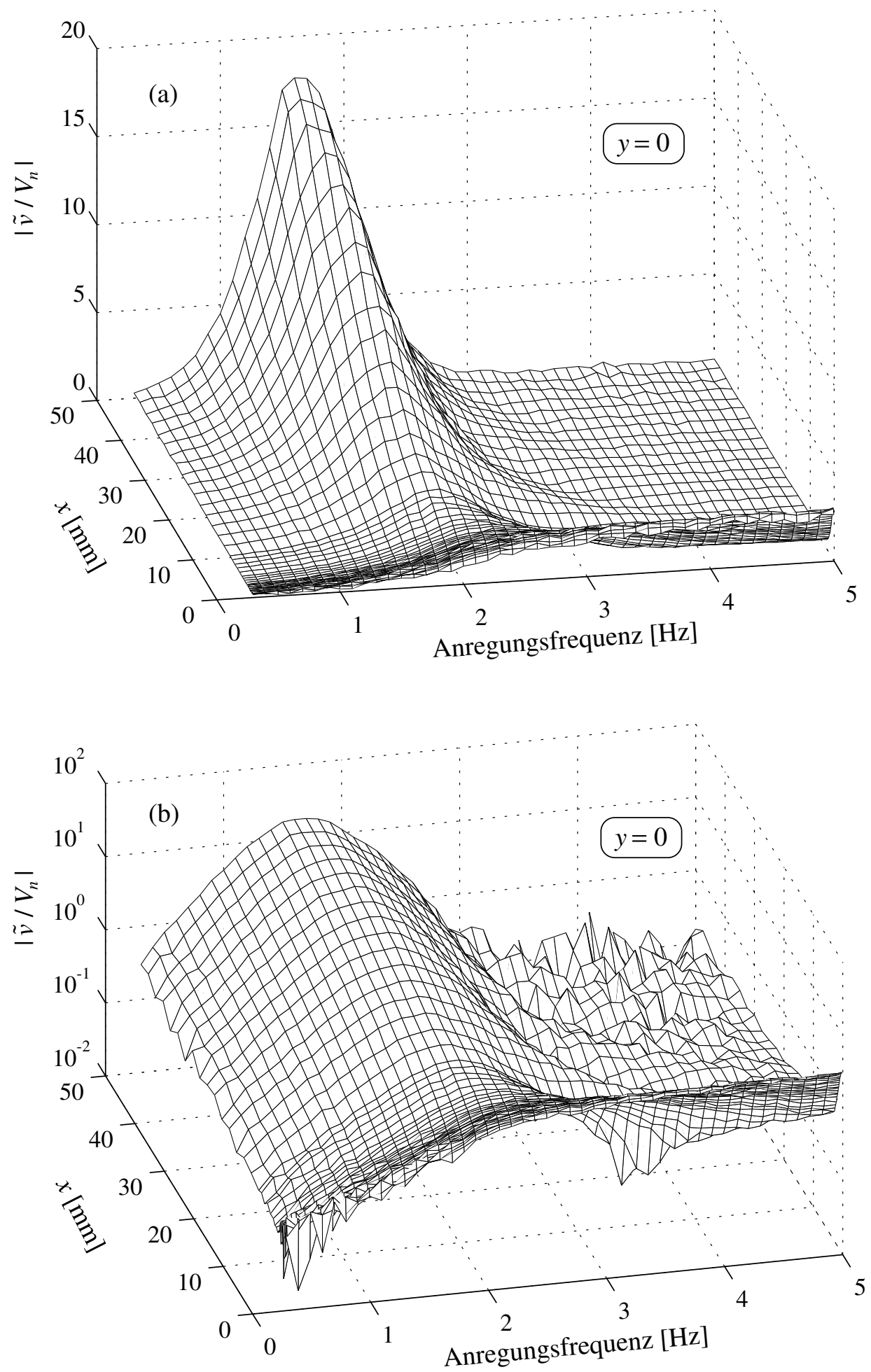

Abb. 4.2: Übersicht über den Betrag der $\tilde{v}$-Komponente bei Anregung mit dem Druckgradienten als Funktion der $x$-Koordinate und der Anregungsfrequenz. (Oben: lineare Auftragung, unten: logarithmische Auftragung) 


$$
\frac{D \tilde{\eta}}{D t}=\frac{\partial \tilde{\eta}}{\partial t}+\bar{u} \frac{\partial \tilde{\eta}}{\partial x}=\tilde{v}, \quad\left(\bar{v}=0, \quad \tilde{u} \frac{\partial \tilde{\eta}}{\partial x} \approx 0 \quad \text { und } \quad \tilde{v} \frac{\partial \tilde{\eta}}{\partial y} \approx 0\right)
$$

Obwohl die $\tilde{v}$-Komponente auch von einem konvektiven Term abhängt, kann man erwarten, dass die Ablösebedingung an der Hinterkante bei einer Darstellung der $\tilde{v}$ Komponente qualitativ zu sehen ist, wenn die Messung an der Stelle stattgefunden hat, wo $\bar{u}$ nur kleine Werte annimmt.

Abbildung 4.2 zeigt den normierten Betrag der gemessenen $\tilde{v}$-Komponente in der Scherschicht bei $y=0 \mathrm{~mm}$ in Abhängigkeit von der $x$-Koordinate und der Anregungsfrequenz. Die Normierungsgröße $V_{n}$ ist die Amplitude der verwendeten DruckgradientenAnregung bei ruhendem Medium am Ort $x=1 \mathrm{~mm}, y=0 \mathrm{~mm}$. Die Anregungsfrequenz liegt zwischen $0.3 \mathrm{~Hz}$ und $5.0 \mathrm{~Hz}$, die Messung ist für verschiedene $x$-Koordinate durchgeführt worden.

Man erkennt zum einen die angefachte Kelvin-Helmholtz-Welle im Frequenzbereich zwischen $0.3 \mathrm{~Hz}$ und ca. $3 \mathrm{~Hz}$, die mit steigender $x$-Koordinate exponentiell anwächst. Dieser Frequenzbereich stimmt ungefähr mit dem Bereich überein, in dem die theoretische Instabilitätswelle ausbreitungsfähig ist (siehe Abbildung 4.1). Bei einer Anregungsfrequenz von ca. $1.8 \mathrm{~Hz}$ ist die Anfachung am größten. Die Instabilitätswelle ist bei $f>3 \mathrm{~Hz}$ nicht mehr anregbar. Zum anderen kann man in der Nähe der Hinterkante jedoch noch nicht von einer echten Kelvin-Helmholtz-Welle sprechen. Im Bereich direkt stromab der Hinterkante ist die $\tilde{v}$-Komponente bei tiefen Frequenzen sehr klein, während sie sich mit wachsender Anregungsfrequenz einem konstanten Wert annähert. Zur Untersuchung der instationären Ablösung von einer halbunendlichen Platte wurden in einigen theoretischen Arbeiten (z. B. Bechert \& Michel [2], Orszag \& Crow [18], Möhring [16] und Daniels [7]) verschiedene Formen der Scherschichtauslenkung diskutiert. Deren Gültigkeit in Abhängigkeit von der Strouhalzahl wurde in Experimenten (z. B. Bechert \& Stahl [5], Satyanarayana \& Davis [21], Poling \& Telionis [19] und GRAF [13]) ermittelt.

GRAF betrachtete dabei die Dynamik der Strömungsablösung als WirbeltranportProblem. Im stationären Fall stellt sich die Wirbelverteilung gemäß der KuttaJoukowski-Hypothese oder kurz Kutta-Bedingung so ein, dass eine auf die Kante extrapolierte äußere Potenzialströmung die geringst mögliche Singularität aufweist. In dem Fall einer überströmten Platte besagt die Kutta-Bedingung somit, dass sich die Strömung tangential an der Hinterkante ablöst. Man kann davon ausgehen, dass bis zur Einstellung dieser Gleichgewichtsverteilung der Wirbeligkeit eine endliche Zeit vergeht. Stört man die Strömung bei Frequenzen, bei denen die Schwingungsperiode nicht mehr groß gegen diese charakteristische Zeit ist, wird es Abweichungen von der quasistationären Kutta-Bedingung geben.

In Abbildung 4.3 sind nun die Werte der gemessenen $\tilde{v}$-Komponenten, normiert mit dem bei ruhendem Medium durch den Druckgradienten angeregten Feld $\tilde{v}_{A n F}^{D r}$ am jeweiligen Messort, als Funktion der Strouhalzahl für vier verschiedene $x$ aufgetragen. Der obere Index $D r$ bedeutet dabei, dass das Wechselfeld durch die Druckgradienten-Anregung erzeugt ist (bei der Schwingkanten-Anregung ist er $K l$ ).

Bei $x=1 \mathrm{~mm}$, außerhalb der Stokesschicht, deren Dicke bei $0.3 \mathrm{~Hz}$ ca. $1.0 \mathrm{~mm}$ und bei $5.0 \mathrm{~Hz}$ ca. $0.25 \mathrm{~mm}$ beträgt, kann man über die instationäre 

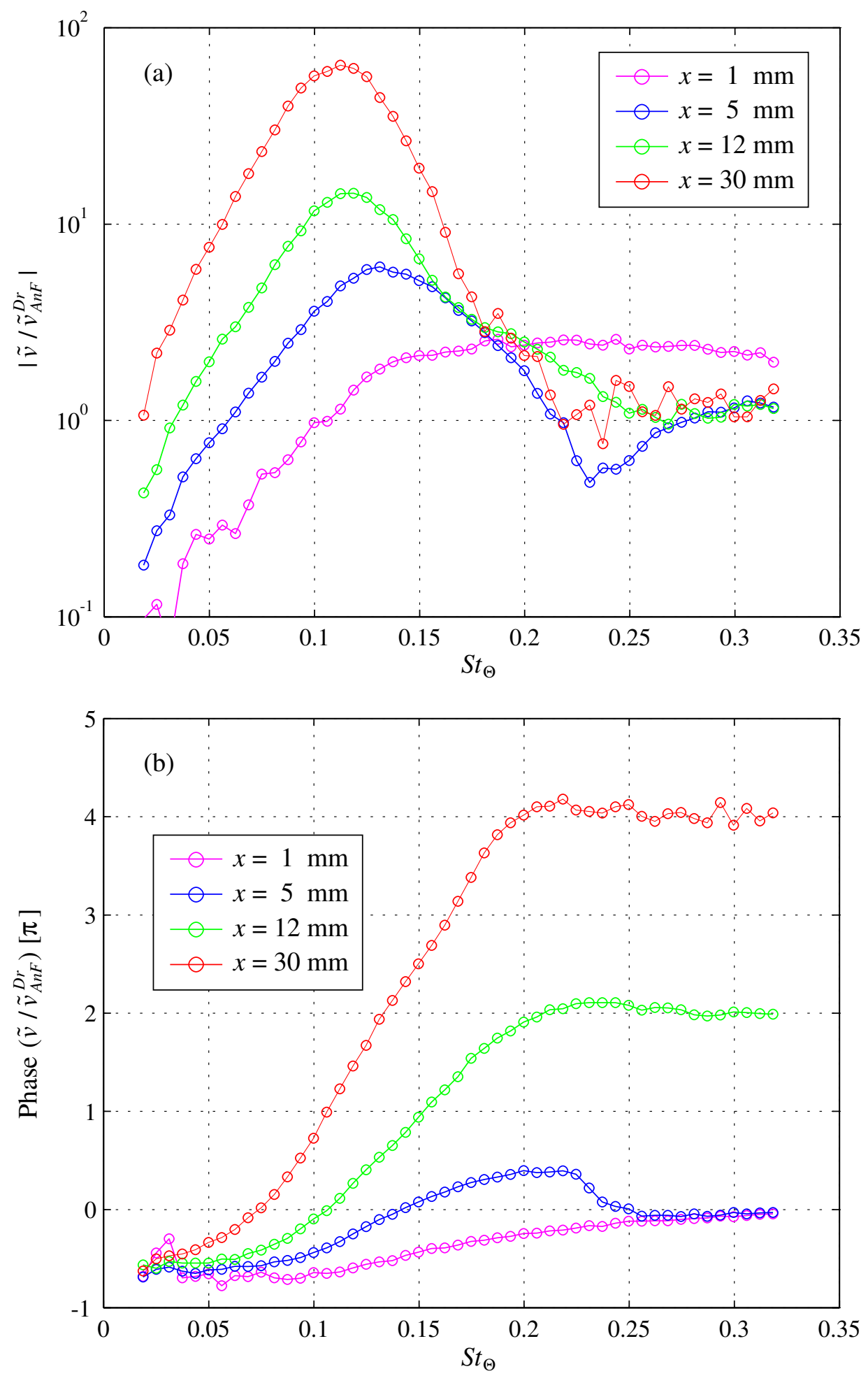

Abb. 4.3: Betrag und Phase der $\tilde{v}$-Komponente als Funktion der Strouhalzahl bei $y=0 \mathrm{~mm}$ für verschiedene $x$-Koordinaten: (a) Betrag und (b) Phase 
Strömungsablösung diskutieren. Für sehr tiefe Frequenzen erwartet man eine quasistationäre Strömungsablösung. Die Strömung löst dabei zu jedem Zeitpunkt tangential von der Hinterkante der Splitterplatte ab, die $\tilde{v}$-Komponente wird daher stromab der Hinterkante sehr klein. Die im Experiment bestimmte $\tilde{v}$-Komponente nimmt für sinkende Strouhalzahlen in der Tat immer weiter ab und wird in dem hier betrachteten Strouhalzahlbereich schon um eine Größenordnung kleiner als das anregende Feld $\tilde{v}_{A n F}^{D r}$. Eine Aussage über die Grenze des Gültigkeitsbereiches der quasistationären KuttaBedingung ist allerdings schwierig, da sich die $\tilde{v}$-Komponete stetig mit Strouhalzahlen ändert. Laut BECHERT ist die quasistationäre Kutta-Bedingung bei Strouhalzahlen von maximal 0.05 gültig.

Bei hohen Strouhalzahlen streben die Werte gegen das anregende Feld, in Abbildung 4.3 geht also der Betrag gegen den Wert eins und die Phase gegen null. Da bei sehr hohen Frequenzen für die Umverteilung der an der Oberfäche der Splitterplatte erzeugte Wirbeligkeit nicht genügend Zeit bleibt und somit das durch den Druckgradienten erzeugte Potenzialfeld nicht abgebaut wird, sollte das Wechselströmungsfeld asymptotisch dem des anregenden Feldes entsprechen. Während die magenta-farbene Kurve (für $x=1 \mathrm{~mm}$ ) in Abbildung 4.3 (a) für $S t_{\Theta}>0.1$ über eins verläuft und mit steigender Strouhalzahl etwa bei einem konstanten Wert bleibt, wurde durch kürzlich durchgeführte numerische Untersuchungen gezeigt, dass $\left|\tilde{v} / \tilde{v}_{A n F}^{D r}\right|$ bei $S t_{\Theta}>0.25$ allmählich abnimmt und erst bei $S t_{\Theta} \approx 0.6$ eins wird (SCHMitz [23]).

Für große $x$ ist eine starke Überhöhung von $\tilde{v} / \tilde{v}_{A n F}^{D r}$ über eins zu erkennen, die bei $x=1 \mathrm{~mm}$ noch nicht auftritt. Diese lässt sich durch die im diesem Frequenzbereich angeregte Kelvin-Helmholtz-Welle erklären. Für $S t_{\Theta}>0.23$ ist die Instabilitätswelle nicht mehr anregbar und die $\tilde{v}$-Komponente nähert sich dem Wert für ein ruhendes Medium. In einem Bereich der Strouhalzahl von ca. 0.18 bis 0.23 sieht man Interferenzen zwischen der Instabilitätswelle und dem anregenden Feld, da die Instabilitätswelle in diesem Bereich nur noch schwach anwächst und ihre Wellenlänge vergleichbar mit der dargestellten $x$-Koordinate ist (Abbildung 4.3 (a)).

Es stellt sich nun die Frage, wo eigentlich die Instabilitätswelle anfängt sich stromab der Hinterkante auszubreiten. Bei $x=1 \mathrm{~mm}$ sieht man noch keine Struktur der Kelvin-Helmholtz-Welle, bzw. das Strömungsfeld hängt vermutlich an dieser Stelle hauptsächlich von der Dynamik der instationären Strömungsablösung ab. Bei $x=5 \mathrm{~mm}$ dagegen ist das Strömungsfeld schon durch die konvektive Instabilität dominiert, wobei es auch einen Übergangsbereich von der instationären Kutta-Bedingung zur Ausbreitung der Instabilitätswelle geben sollte. Zur Beantwortung dieser Frage ist es sicherlich praktisch, das gesamte Wechselströmungsfeld zu zerlegen, damit man das Nahfeld bzw. das Feld der reinen KH-Welle quantitativ untersuchen kann. Ein solches Vorgehen, das in Abschnitt 4.2 weiter diskutiert wird, könnte auch bei der Suche nach einer Nahfeldgröße helfen, die die Amplitude der Kelvin-Helmholtz-Welle festlegt.

\subsubsection{Vergleich der Dispersionsbeziehung mit Messwerten}

Abbildung 4.4 zeigt einen Vergleich der gemessenen Dispersionskurven mit den theoretischen Kurven für das entsprechende Strömungsprofil. Da das Gleichströmungsprofil 


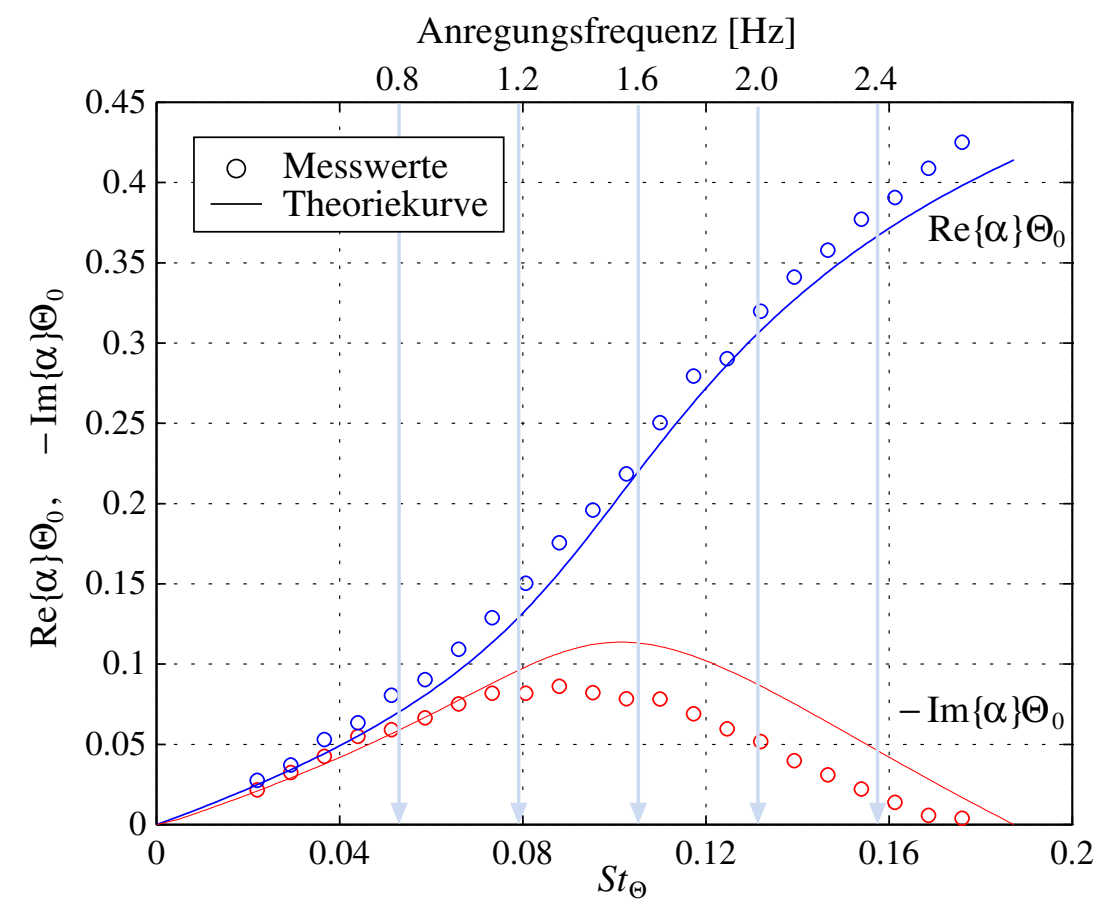

Abb. 4.4: Vergleich zwischen Theorie und Experiment: Normierte Phasenkonstante $\operatorname{Re}\{\alpha\}$ und Anfachungskonstante $\operatorname{Im}\{\alpha\}$ der Instabilitätswelle in der Scherschicht als Funktion von $S t_{\Theta}$

von der $x$-Koordinate und der Schwingungsamplitude abhängt, ist auch die komplexe Wellenzahl von diesen Paramentern abhängig.

Für die theoretische Wellenzahl wurde das Strömungsprofil der ungestörten Scherschicht bei $x=30 \mathrm{~mm}$ festgelegt. Aus der gemessenen Wechselgeschwindigkeit kann die komplexe Wellenzahl $\alpha$ mit Hilfe der Gleichung (4.2) berechnet werden. Dabei wird angenommen, dass die Wechselgeschwindigkeiten klein gegenüber der Gleichgeschwindigkeit in Hauptströmungsrichtungsrichtung sind. Die Inhomogenität des Gleichströmungsfeldes wird vernachlässigt.

Wie man in Abbildung 4.4 sieht, gibt es bei niedrigen Strouhalzahlen sowohl für die Anfachungs- als auch die Phasenkonstante eine gute Übereinstimmung zwischen den experimentellen und den theoretischen Werten. Es gibt allerdings eine systematische Abweichung zwischen ihnen bei der Phasenkonstante $\operatorname{Re}\{\alpha\}$, und diese Abweichung zeigt, dass die aus der Messung bestimmte Wellenlänge kleiner ist als die theoretische Wellenlänge, während sie aber eine ähnliche Abhängigkeit von der Strouhalzahl zeigt. Die theoretisch bestimmte Grenze der Strouhalzahl $S t_{\Theta, \text { the }}^{\max } \approx 0.19$, bis zu der KHWellen ausbreitungsfähig sind, wird im Experiment nicht erreicht. Die experimentelle $S t_{\Theta, \text { exp }}^{\max }$ lässt sich aus Abbildung $4.4 \mathrm{zu}$ etwa 0.17 extrapolieren, was einer Frequenz von ca. $2.6 \mathrm{~Hz}$ entspricht. Freymuth z. B. konnte in seinem Experiment (FREYMUTH [10]) auch nur bis $S t_{\Theta, \text { exp }}^{\max } \approx 0.16$ eine Anfachung nachweisen, wobei für das Gleichströmungsprofil ein tanh-Profil (Gleichung (3.2)) angenommen wurde und dessen theoretische $S t_{\Theta, \text { the }}^{\max }$ nach Michalke (Michalke [15]) 0.25 beträgt. Der Grund, weshalb die 


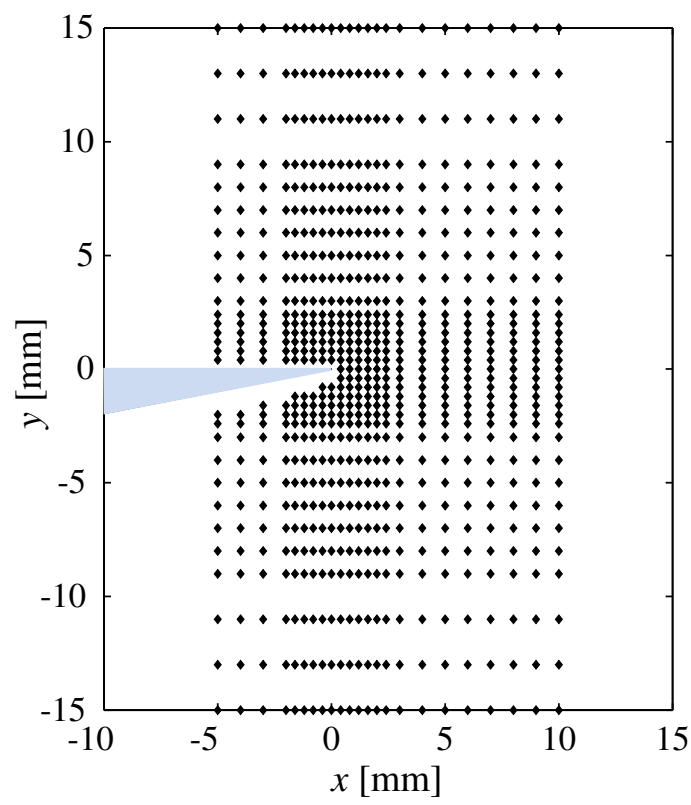

Abb. 4.5: Übersicht über das Messpunktgitter im Bereich der Ablösekante

experimentell bestimmte Grenze nicht die theoretische Grenze erreichen kann, könnte an der im Experiment immer vorhandenen Inhomogenität des $\bar{u}$-Profils durch die Aufweitung der Scherschicht liegen, die in der hier zitierten theoretischen Arbeit noch nicht berücksichtigt wurde. Diese Inhomogenität ist gerade im Ablösebereich der Scherschicht am stärksten, wo diese am empfindlichsten auf äußere Störungen reagiert.

\subsection{Untersuchung des instationären Strömungsfel- des}

An der experimentell bestimmten Dispersionsbeziehung hat man gesehen, dass die von der Hinterkante der Splitterplatte abgelösete Scherschicht bis ca. $2.6 \mathrm{~Hz}\left(\operatorname{Sr}_{\Theta, \text { exp }}^{\max } \approx 0.17\right)$ anregbar ist. Die Eigenschaften der Kelvin-Helmholtz-Instabilitätswellen werden in diesem Frequenzbereich experimentell untersucht. Die bei der Messung verwendeten Frequenzen sind nun unabhängig von den Anregungsmechanismen $0.8 \mathrm{~Hz}, 1.2 \mathrm{~Hz}, 1.6 \mathrm{~Hz}$, $2.0 \mathrm{~Hz}$ und $2.4 \mathrm{~Hz}$, die den Bereich gleichmäßig überdecken, in dem die KH-Wellen ausbreitungsfähig sind (siehe Pfeile in Abbildung 4.4). Messungen wurden in einer $x$-y-Ebene im Nahbereich der Hinterkante durchgeführt, so dass man nicht nur die angeregte Scherschicht, sondern auch instationäre Strömungsablösungen untersuchen kann. Abbildung 4.5 zeigt das zugehörige Messpunktgitter; der minimale Punktabstand im Bereich großer Geschwindigkeitsgradienten beträgt $0.4 \mathrm{~mm}$. 


\subsubsection{Zerlegung des Messfeldes}

Zur Untersuchung der Wechselwirkungen zwischen dem Gleichströmungsfeld und dem anregenden Feld soll zuerst ein Überblick über das zu untersuchende Wechselgeschwindigkeitsfeld mit Hilfe der Stromfunktion gegeben werden. Das gesamte Wechselfeld $\tilde{\psi}_{\text {All }}(x, y)$ besteht mindestens aus dem Feld der KH-Wellen $\tilde{\psi}_{K H}(x, y)$ und dem anregenden Feld $\tilde{\psi}_{A n F}(x, y)$. Ein unbekanntes Feld, das nicht zu diesen beiden Feldern gehört und zur Anregung kohärent ist, wird hier als (eigentliches) Nahfeld $\tilde{\psi}_{N F}(x, y)$ bezeichnet. Das Wechselfeld $\tilde{\psi}_{\text {All }}(x, y)$ wird also in der folgenden Zerlegung beschrieben:

$$
\tilde{\psi}_{A l l}(x, y)=\tilde{\psi}_{A n F}(x, y)+\tilde{\psi}_{K H}(x, y)+\tilde{\psi}_{N F}(x, y),
$$

Da das anregende Feld $\tilde{\psi}_{A n F}$, das entweder durch die Druckgradienten- oder die Schwingkanten-Anregung bei ruhendem Medium erzeugt wird, mittels einer numerischen Rechnung genau beschrieben wird (siehe Anhang A), kann das durch numerische Simulation bestimmte Feld $\tilde{\psi}_{A n F}$ von dem gesamten Feld $\tilde{\psi}_{\text {All }}$ abgezogen werden. Man erhält nun das Wechselfeld $\tilde{\psi}_{K H+N F}$, das nur aus $\tilde{\psi}_{K H}$ und $\tilde{\psi}_{N F}$ besteht:

$$
\tilde{\psi}_{K H+N F}(x, y):=\tilde{\psi}_{A l l}(x, y)-\tilde{\psi}_{A n F}(x, y)=\tilde{\psi}_{K H}(x, y)+\tilde{\psi}_{N F}(x, y) .
$$

Da die Dynamik der freien Scherschicht außerhalb des Kantenbereichs durch die angefachte KH-Welle dominiert wird, wird das Fernfeld von $\tilde{\psi}_{K H+N F}$ durch einen einzigen Freiheitsgrad, d.h. durch einen einzigen räumlichen Verlauf der KH-Instabilitätswelle beschrieben, lediglich die komplexe Amplitude bleibt frei.

Zur Bestimmung des Nahfeldes $\tilde{\psi}_{N F}$ braucht man allerdings den räumlichen Verlauf der $\mathrm{KH}-$ Welle in der Nähe der Hinterkante, wobei auch die dazugehörige gedämpfte KHWelle nicht vernachlässigt werden kann. $\tilde{\psi}_{K H}$ wird daher mit den zwei Lösungen (eine entspricht der angefachten-, die andere der gedämpften Mode), die durch Integration der Rayleigh-Gleichung (4.1) mittels eines Runge-Kutta-Verfahrens gewonnen wurden, folgendermaßen beschrieben:

$$
\tilde{\psi}_{K H}(x, y)=C_{A} \psi_{0}(y) \exp (i \alpha x)+C_{D} \psi_{0}^{*}(y) \exp \left(i \alpha^{*} x\right)
$$

wobei $C_{A}$ einer komplexen Amplitude für die angefachte Lösung entspricht und $C_{D}$ für die gedämpfte. Die zueinander konjugiert komplexen Wellenzahlen $\alpha$ und $\alpha^{*}$ sind die Eigenwerte der beiden Lösungen, $\operatorname{Im}\{\alpha\}$ ist dabei negativ. Dabei ist Gleichung (4.7) nur eine lokale Beschreibung, die von der Homogenität des Strömungsprofils ausgeht. Später (Abschnitt 4.2.3) werden $\psi_{0}, \alpha, C_{A}$ und $C_{D}$ noch schwach von der $x$-Koordinate abhängen.

Da es eine direkte Beziehung zwischen der Stromfunktion und der Strömungsgeschwindigkeit gibt (siehe Gleichung (4.2)), werden im folgenden drei Wechselgeschwindigkeitsfelder ausführlicher dargestellt. Die Anregungsfrequenzen sind dabei $0.8 \mathrm{~Hz}, 1.6 \mathrm{~Hz}$ und $2.4 \mathrm{~Hz}$. Um einen besseren Vergleich zu ermöglichen, wurden alle Störgeschwindigkeiten bei der Druckgradienten-Anregung mit der Amplitude $V_{n}$ und bei der Schwingkanten-Anregung mit $V_{k}$ normiert, die folgendermaßen definiert sind: 


$$
V_{n}=\tilde{v}_{A n F}^{D r}\left(d_{0}, 0\right), \quad V_{k}=\tilde{v}_{A n F}^{K l}(0,0)
$$

$d_{0}$ ist dabei eine für das durch den Druckgradienten erzeugte anregende Feld charakteristische Länge, die hier mit $d_{0}=1 \mathrm{~mm}$ festgelegt wurde, da die Potenzialströmungsfelder selbstähnlich sind und man sich bei $x=1 \mathrm{~mm}$ in dem untersuchten Frequenzbereich genügend weit außerhalb der Stokesschicht befindet.

$v$-Komponente

Die Abbildungen 4.6 bis 4.8 zeigen den Betrag der mit $V_{n}$ normierten komplexen Amplitude $\tilde{v}$ in einer dreidimensionalen Darstellung für das Gesamtfeld $\tilde{v}_{A l l}^{D r}$ und das Feld ohne das anregende Feld, also $\tilde{v}_{K H+N F}^{D r}$. Der Blick ist - wie auch in den noch folgenden 3D-Graphen - jeweils stromab gerichtet, und die Hauptströmung befindet sich links von der Nebenströmung.

Obwohl die Anregungsfrequenz $0.8 \mathrm{~Hz}\left(S t_{\Theta} \approx 0.05\right)$ nicht mehr in dem Bereich ist, in dem von der Gültigkeit der quasistationären Kutta-Bedingung ausgegangen werden kann, sieht man immer noch in Abbildung 4.6 (a), dass $\tilde{v}_{A l l}^{D r}$ in der Nähe der Hinterkante unterdrückt ist. Der räumliche Verlauf der Kelvin-Helmholtz-Welle ist bei der Darstellung sehr schwierig zu erkennen, da einerseits die zu dieser Strouhalzahl entsprechende Anfachungskonstante gering ist, andererseits die Wellenlänge der Instabilitätswelle sehr groß im Vergleich zur Länge des Messgebietes $\left(x_{\max }=10 \mathrm{~mm}\right)$ ist. Man sieht allerdings, dass der Betrag im Potenzialbereich besonders bei $y=15 \mathrm{~mm}$ mit steigender $x$-Koordinate anwächst.

Das Feld $\tilde{v}_{K H+N F}^{D r}$ ist ein überlagertes Feld aus den KH-Wellen $\tilde{v}_{K H}^{D r}$ und dem Nahfeld $\tilde{v}_{N F}^{D r}$. Da im Bereich tiefer Frequenzen (bei denen man noch die Gültigkeit der quasistationären Kutta-Bedingung erwartet), wie schon erwähnt, $\tilde{v}_{A l l}^{D r}$ nahe der Hinterkante sehr klein ist, sind gemäß Gleichung (4.6) die Beträge von $\tilde{v}_{K H+N F}^{D r}$ und $\tilde{v}_{A n F}^{D r}$ gleich und die Phasen entgegengesetzt, wenn die Anregungsfrequenz bzw. die Strouhalzahl gegen null geht. Bei $0.8 \mathrm{~Hz}$ sind die räumlichen Strukturen von $\tilde{v}_{K H+N F}^{D r}$ (Abbildung 4.6 (b)) und $\tilde{v}_{A n F}^{D r}$ (Abbildung A.5 (b)) sehr ähnlich, wobei man jedoch bei $\tilde{v}_{K H+N F}^{D r}$ in eine $x$-Richtung laufende Welle, insbesondere bei (hier nicht dargestellten) der Phase erkennt.

Bei der Druckgradienten-Anregung mit $1.6 \mathrm{~Hz}\left(S t_{\Theta} \approx 0.10\right.$, Abbildung 4.7) sieht man deutlich das voll entwickelte KH-Wellenprofil weiter stromab der Ablösekante. Das Maximum des Profils liegt etwa auf der zentralen Linie $y=0 \mathrm{~mm}$, und der Betrag des Profils stromauf der Hinterkante geht bei $\tilde{v}_{A l l}^{D r}$ nahezu glatt in das Profil der KH-Welle über. Zieht man das anregende Feld $\tilde{v}_{A n F}^{D r}$ von dem gesamten Messfeld $\tilde{v}_{A l l}^{D r}$ ab, so erhält man einen anderen Aspekt des Messfeldes. Nun ist bei dem Feld $\tilde{v}_{K H+N F}^{D r}$ auffällig, dass die $\tilde{v}$-Komponente unmittelbar stromab der Hinterkante auf der zentralen Linie nicht mit steigender $x$-Koordinate kontinuierlich anwächst, sondern zunächst einmal abnimmt und dann erst beginnt exponentiell anzuwachsen. Es entsteht also ein „Peak“, bevor sich die Instabilitätswelle voll entwickelt.

Für die Anregungsfrequenz $2.4 \mathrm{~Hz}\left(S t_{\Theta} \approx 0.16\right)$, Abbildung 4.8, ergibt sich wieder ein anderes Bild. Bei $\tilde{v}_{A l l}^{D r}$ erkennt man eine starke Interferenz zwischen den KH-Wellen 


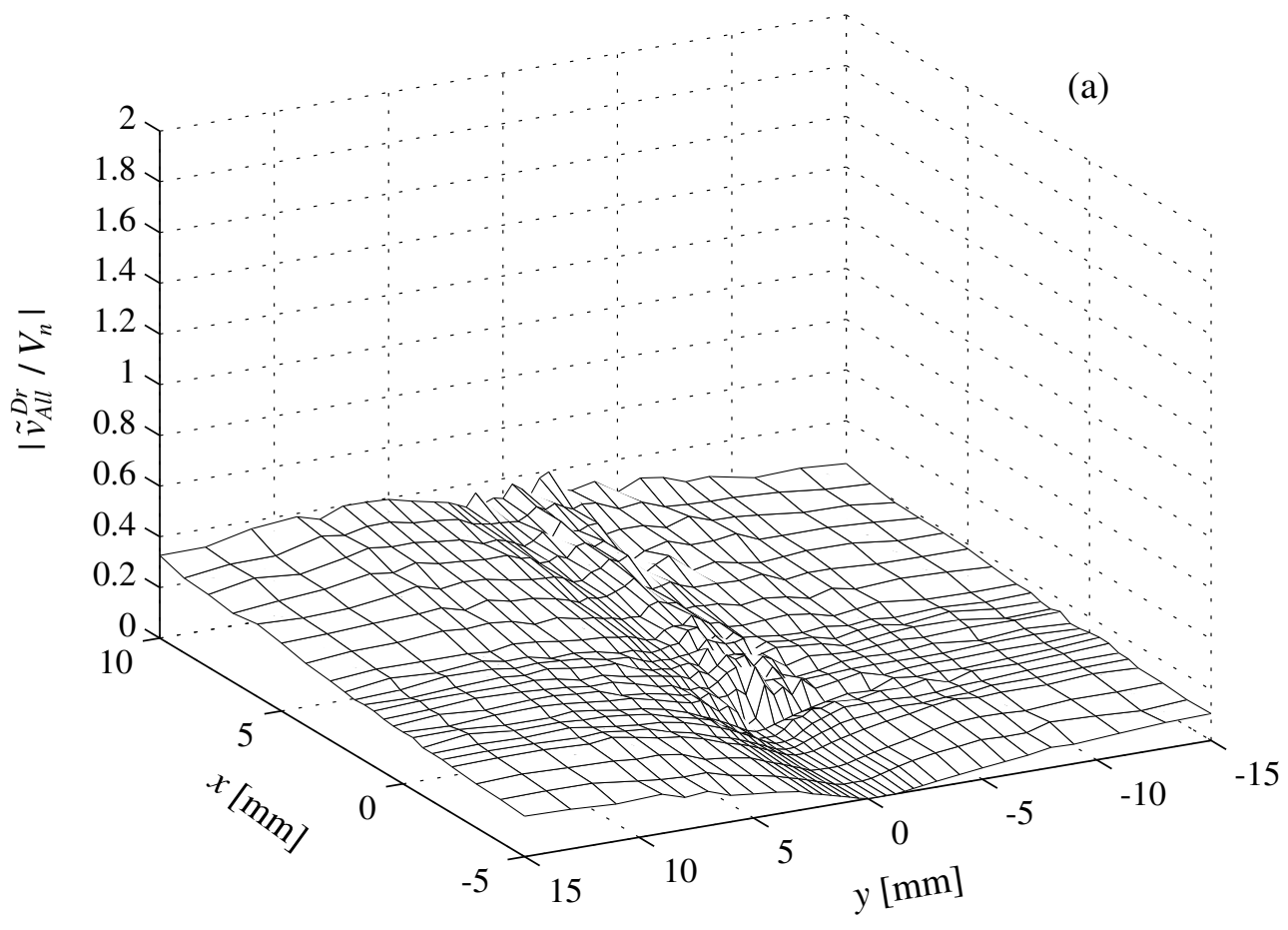

Anregungsfrequenz $=0.8 \mathrm{~Hz}$

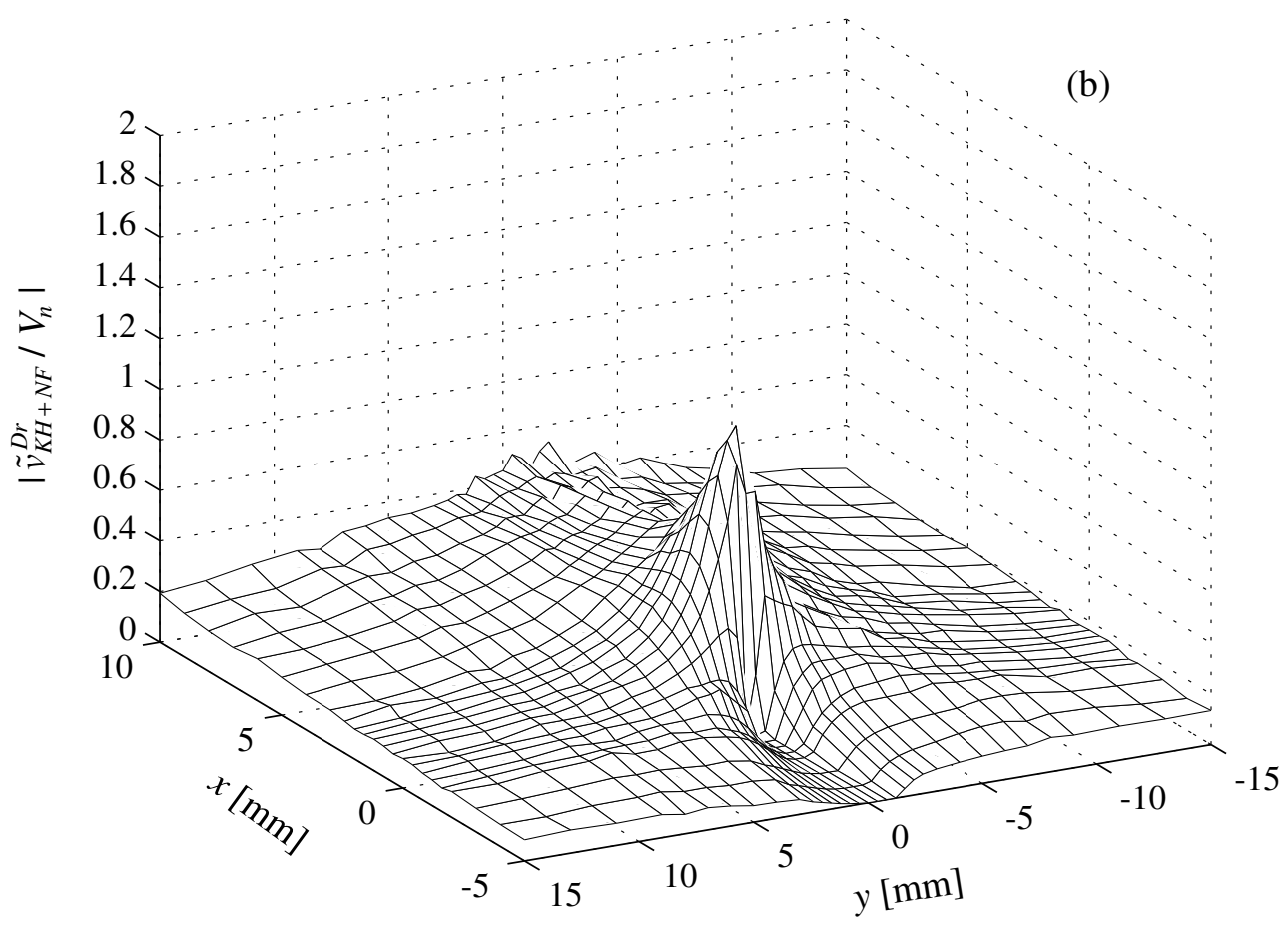

Abb. 4.6: Übersicht über den Betrag der normierten $\tilde{v}$-Komponente der Wechselgeschwindigkeit bei $0.8 \mathrm{~Hz}$ : (a) das Gesamtfeld $\tilde{v}_{A l l}^{D r}$; (b) $\tilde{v}_{K H+N F}^{D r}$ (das anregende Feld ist vom Gesamtfeld abgezogen) 


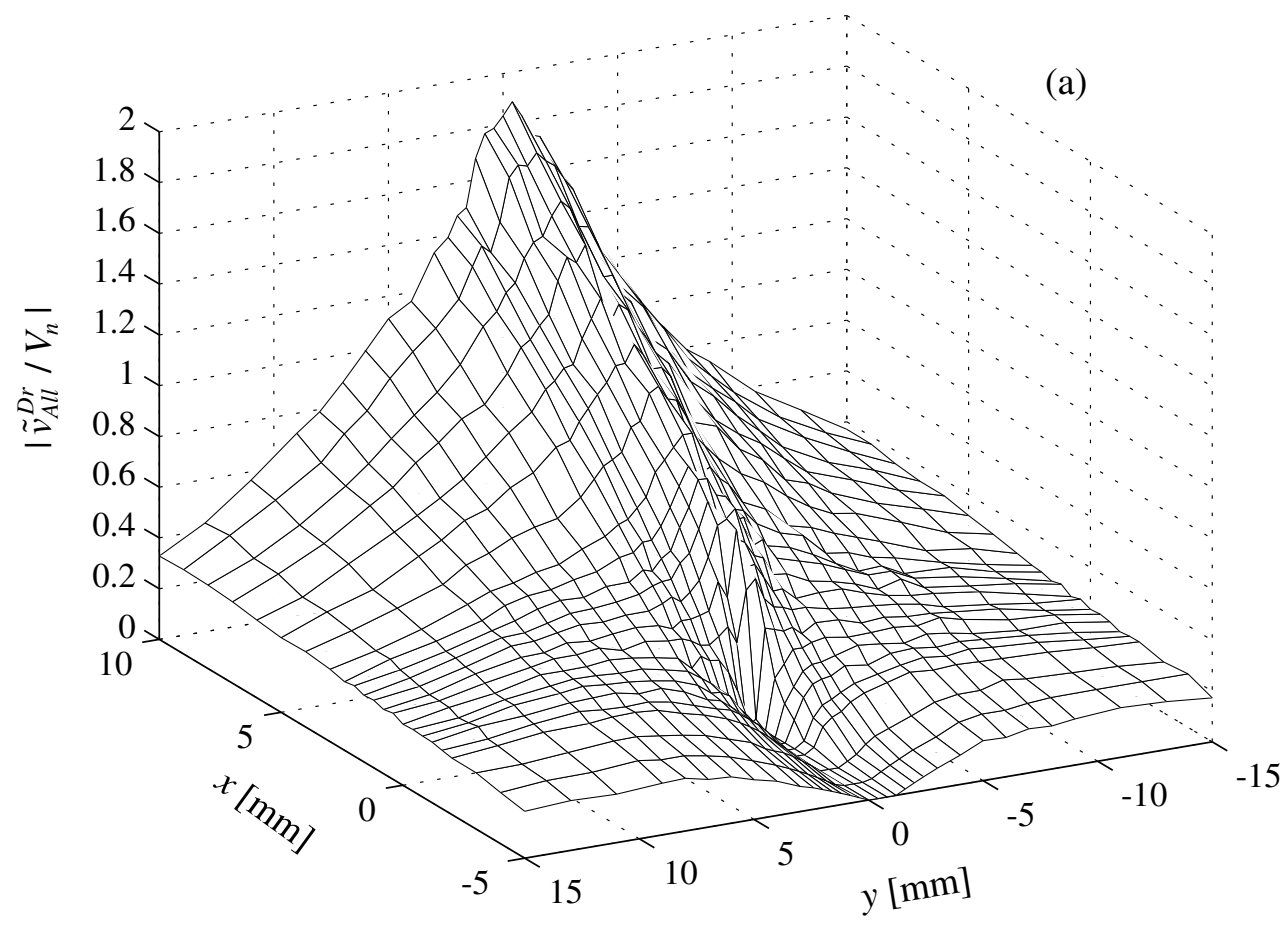

Anregungsfrequenz $=1.6 \mathrm{~Hz}$

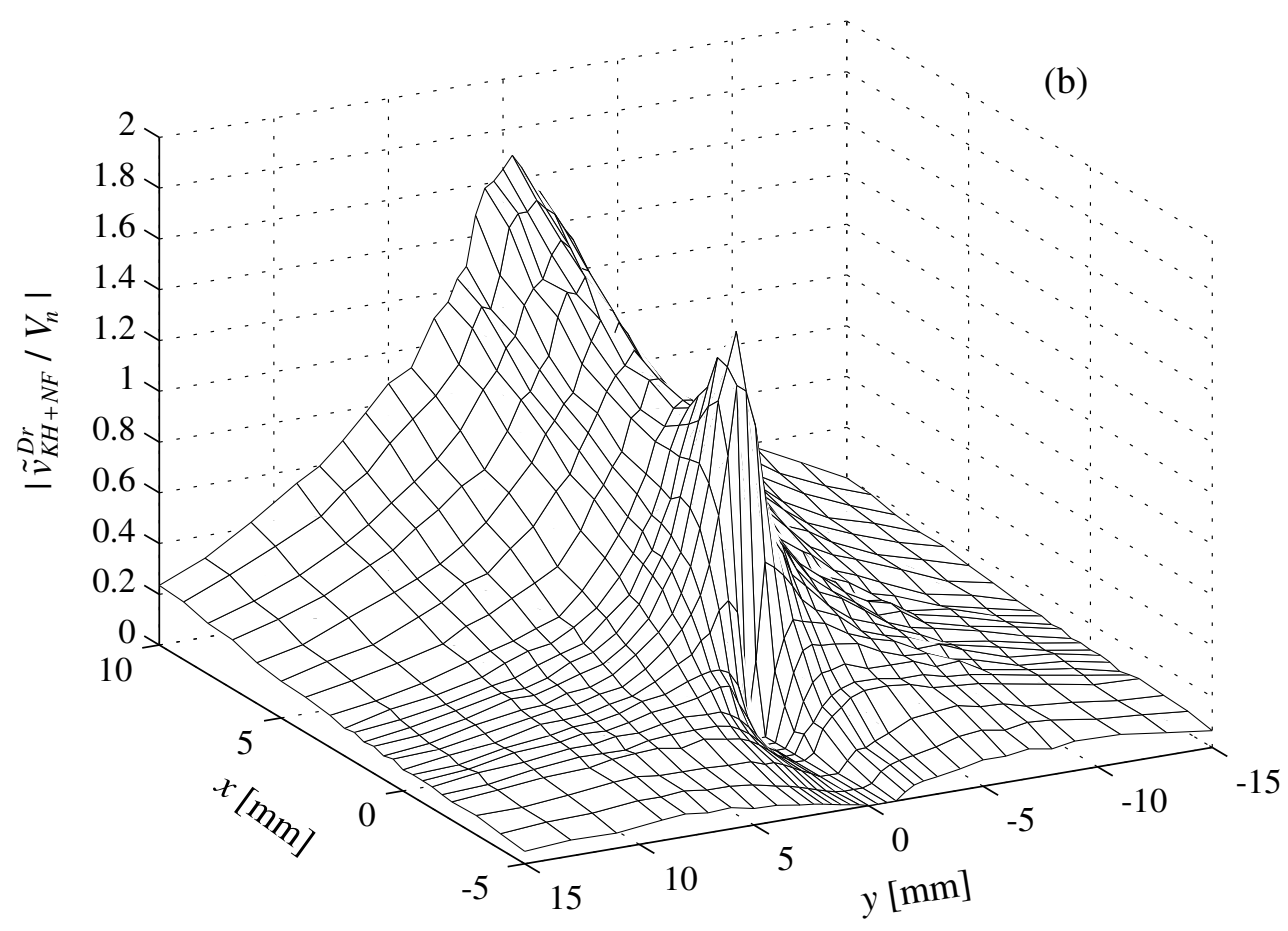

Abb. 4.7: Übersicht über den Betrag der normierten $\tilde{v}$-Komponente der Wechselgeschwindigkeit bei $1.6 \mathrm{~Hz}$ : (a) das Gesamtfeld $\tilde{v}_{A l l}^{D r}$; (b) $\tilde{v}_{K H+N F}^{D r}$ (das anregende Feld ist vom Gesamtfeld abgezogen) 


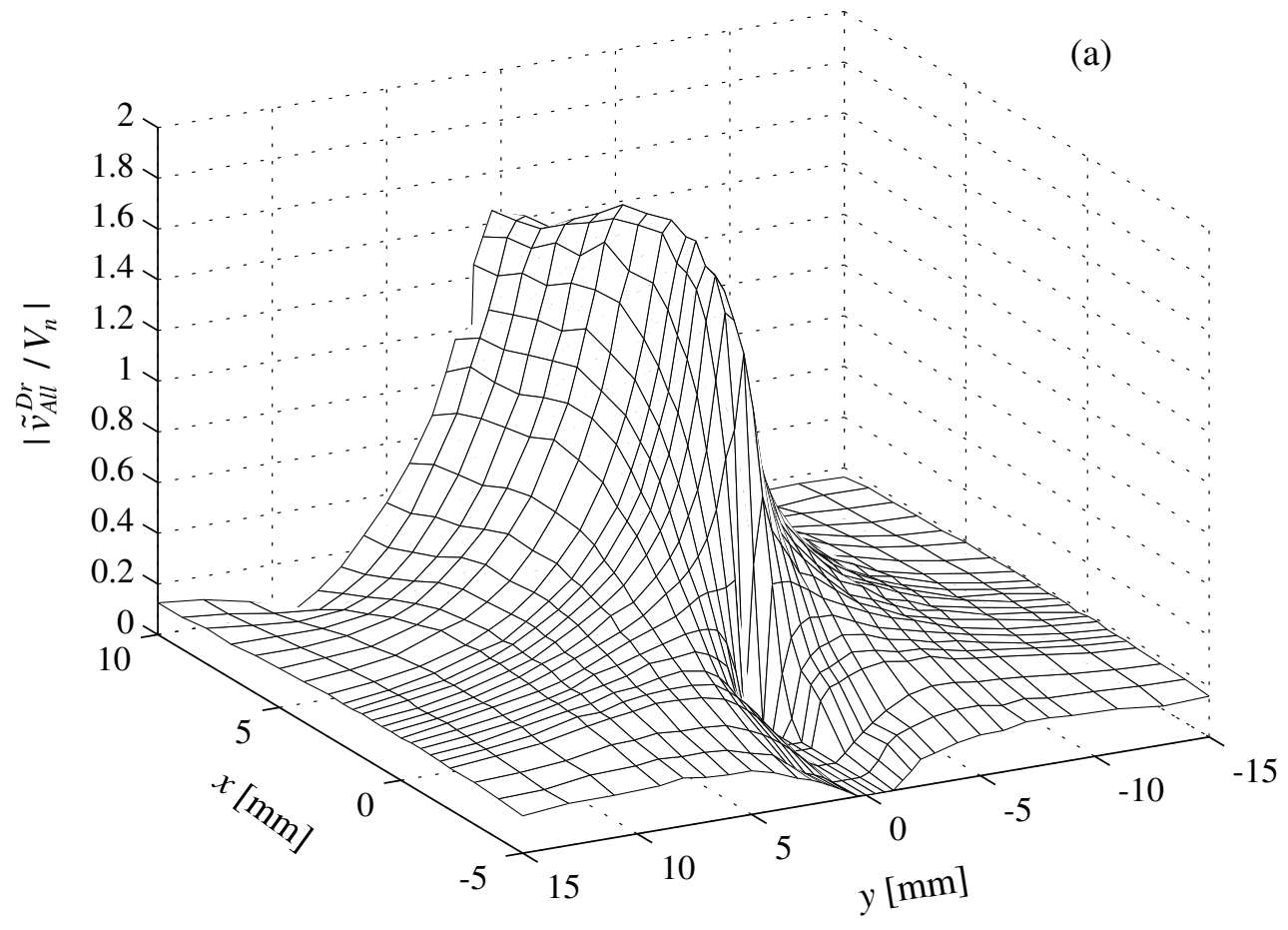

Anregungsfrequenz $=2.4 \mathrm{~Hz}$

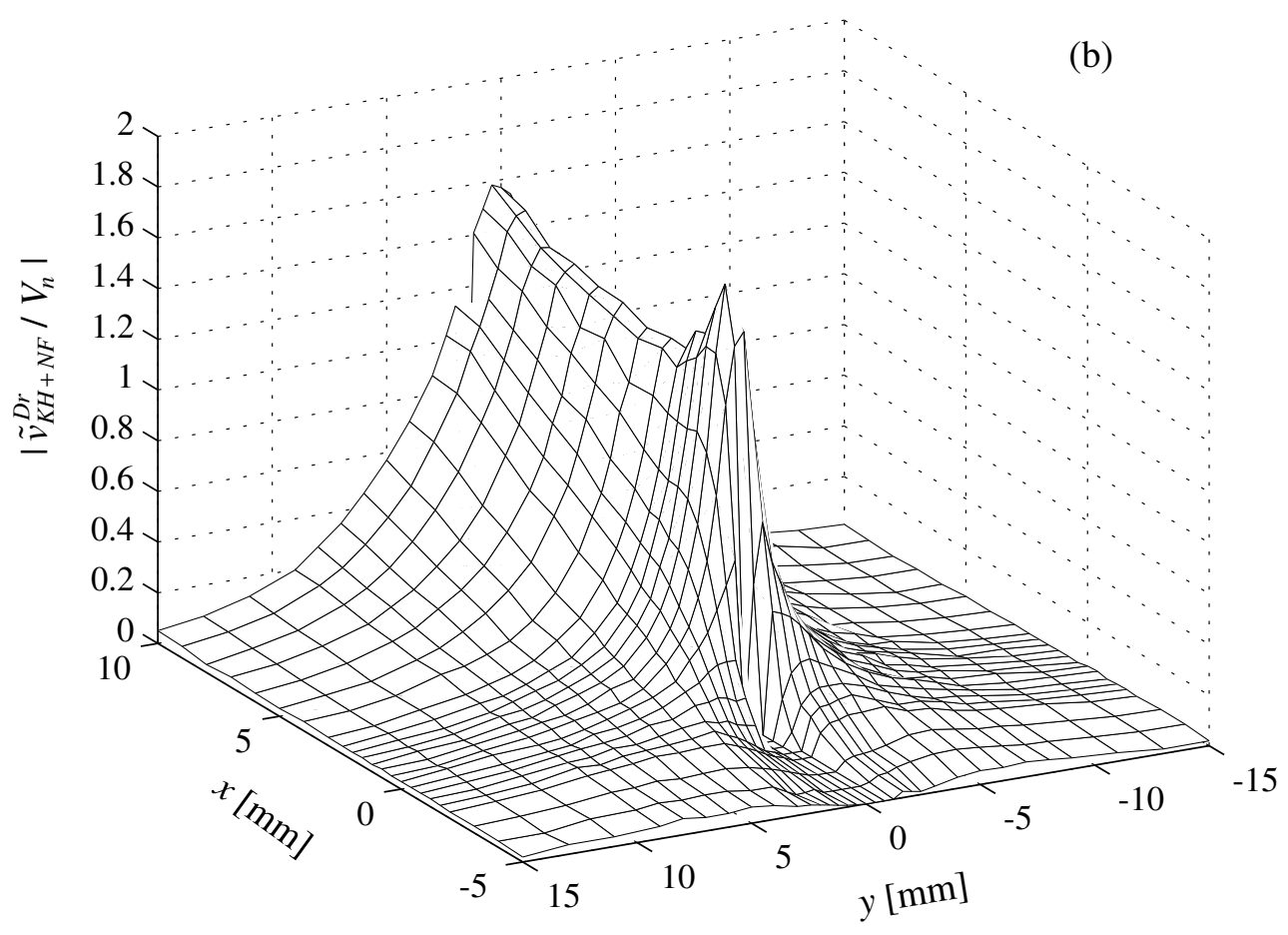

Abb. 4.8: Übersicht über den Betrag der normierten $\tilde{v}$-Komponente der Wechselgeschwindigkeit bei $2.4 \mathrm{~Hz}$ : (a) das Gesamtfeld $\tilde{v}_{A l l}^{D r}$; (b) $\tilde{v}_{K H+N F}^{D r}$ (das anregende Feld ist vom Gesamtfeld abgezogen) 
und dem anregenden Feld. Die angefachte Instabilitätswelle ist daher in Abbildung 4.8 (a) nicht deutlich zu sehen. $\tilde{v}_{K H+N F}^{D r}$ zeigt aber ein entwickeltes Profil der Instabilitätswelle stromab der Ablösekante, deren Anfachungskonstante allerdings kleiner ist als bei $1.6 \mathrm{~Hz}$. Es ist noch anzumerken, dass $\tilde{v}_{K H+N F}^{D r}$ im Bereich der Scherschicht konzentriert ist, da die Beträge der KH-Wellen beim Übergang in den Potenzialbereich und im Potenzialbereich selbst sehr stark abklingen. Außerdem ist $\tilde{v}_{K H+N F}^{D r}$ stromauf der Hinterknate sehr klein. Das bedeutet, dass sich das Gesamtfeld $\tilde{v}_{A l l}^{D r}$ stromauf der Hinterkante aufgrund der Überlagerung einer Gleichströmung nicht wesentlich geändert hat. Der „Peak" direkt stromab der Hinterkante erscheint auch für diese Strouhalzahl. Vergleicht man die Felder von $\tilde{v}_{K H+N F}^{D r}$ für die drei Frequenzen miteinander, so erkennt man eine gewisse Ähnlichkeit zwischen ihnen, besonders im Bereich direkt hinter der Kante. Es lässt sich vermuten, dass diese Struktur, nämlich der „Peak“ vor dem exponentiellen Wachstum der Instabilitätswelle, nicht durch die KH-Welle beschrieben werden kann, sondern dem Nahfeld $\tilde{v}_{N F}^{D r}$ zugeordnet werden muss.

$\underline{u \text {-Komponente }}$

Die instationäre $\tilde{u}$-Komponente, die zu der gerade dargestellten $\tilde{v}$-Komponente gehört, wird im folgenden diskutiert. Die $\tilde{u}$-Komponente und die $\tilde{v}$-Komponente haben aufgrund der Kontinuitätsgleichung einen engen Zusammenhang, die bei den oben vorgestellten Feldern unabhängig voneinander erfüllt sein muss,

$$
\frac{\partial \tilde{u}_{N F}}{\partial x}+\frac{\partial \tilde{v}_{N F}}{\partial y}=0 \quad \Rightarrow \quad \frac{\partial \tilde{u}_{K H+N F}}{\partial x}+\frac{\partial \tilde{v}_{K H+N F}}{\partial y}=0
$$

wobei für die Felder $\tilde{\psi}_{K H}$ und $\tilde{\psi}_{A n F}$ aufgrund der theoretischen Beschreibung keine Möglichkeit zur Verletzung der Kontinuitätsgleichung besteht.

Die Abbildungen 4.9 bis 4.11 zeigen die mit $V_{n}$ normierten Beträge der $\tilde{u}$-Komponente und zwar den Gesamtwert $\tilde{u}_{A l l}^{D r}$ und die Differenz $\tilde{u}_{K H+N F}^{D r}$ zwischen $\tilde{u}_{A l l}^{D r}$ und $\tilde{u}_{A n F}^{D r}$. Das anregende Feld $\tilde{u}_{A n F}^{D r}$ wird durch die numerische Simulation bestimmt und ihr Betrag ist in Abbildung A.5 (a) gezeigt (siehe Anhang A). Da sich die räumliche Verteilung des anregenden Felds $\tilde{u}_{A n F}^{D r}$ hauptsächlich auf den Bereich stromauf der Hinterkante konzentriert, erkennt man einen deutlichen Unterschied zwischen $\tilde{u}_{A l l}^{D r}$ und $\tilde{u}_{K H+N F}^{D r}$ für alle Strouhalzahlen im Bereich stromauf der Hinterkante.

Bei der Anregungsfrequenz $0.8 \mathrm{~Hz}$ und entsprechend geringer Anfachung geht der Betrag von $\tilde{u}_{A l l}^{D r}$ stromauf der Hinterkante glatt in die Scherschicht über. Das Geschwindigkeitsprofil ändert sich also kaum mit steigender $x$-Koordinate. Da $\tilde{u}_{A l l}^{D r}$ im Nebenströmungsbereich stark unterdrückt ist, stellt man für diese Strouhalzahl fest, dass sich die Strömung immer noch von der Hinterkante tangential ablöst. Die Veränderung vom Feld $\tilde{u}_{A l l}^{D r}$ zum Feld $\tilde{u}_{K H+N F}^{D r}$ ist nur im Bereich $x<0$ zu sehen. Man erkennt jedoch erst bei $\tilde{u}_{K H+N F}^{D r}$ einen kleinen "Peak“ im Nebenströmungsbereich unmittelbar an der Hinterkante, den man auch bei $\tilde{v}_{K H+N F}^{D r}$ beobachten konnte.

Auch für den Fall der stärksten Anfachung bei der Frequenz $1.6 \mathrm{~Hz}$ erfolgt der glatte Übergang beim Betrag $\tilde{u}_{A l l}^{D r}$. Es ist zu sehen, dass sich das KH-Wellenprofil weiter stromab der Ablösekante voll entwicklt hat. Für diese Strouhalzahl erkennt man das 


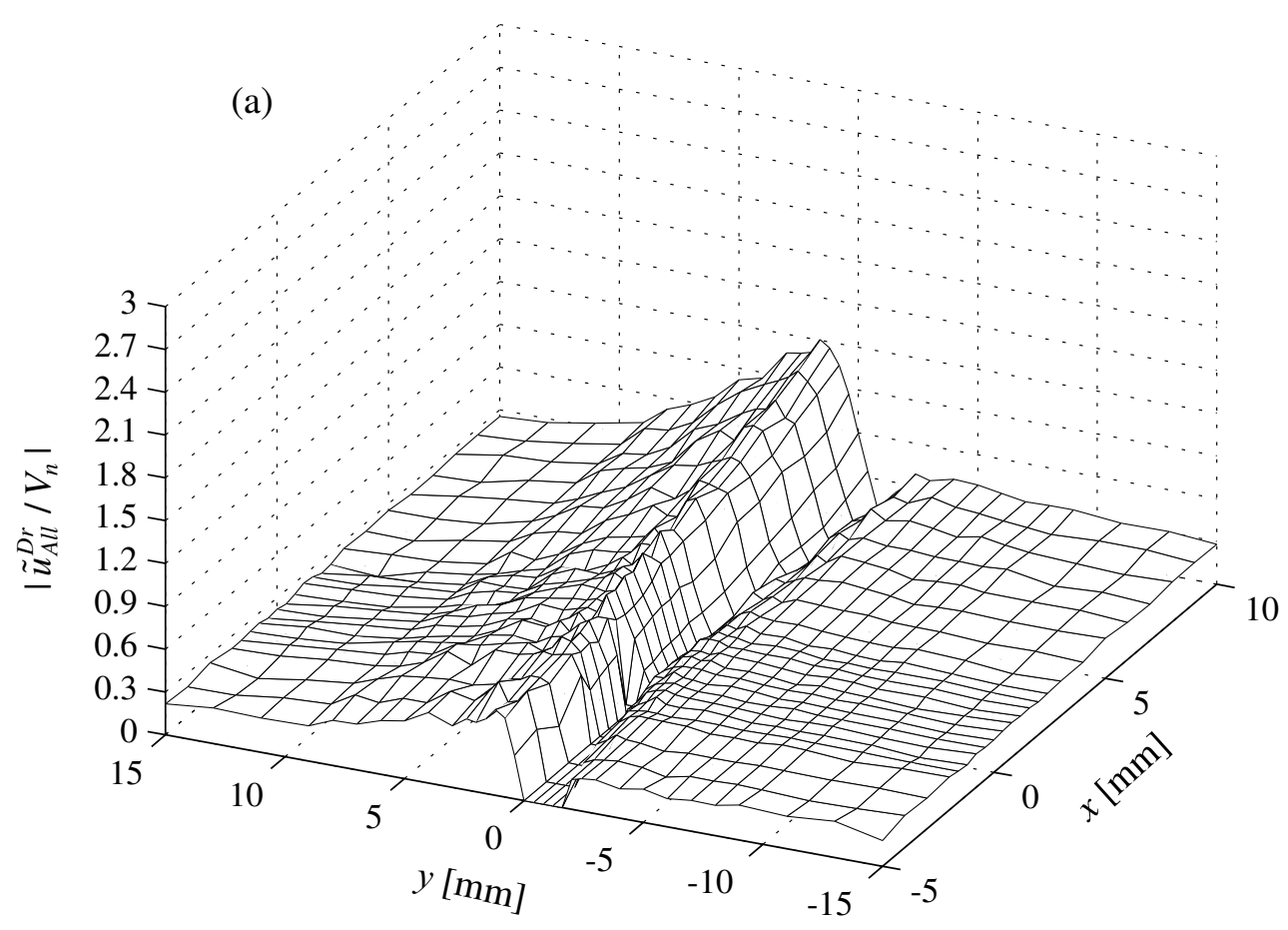

Anregungsfrequenz $=0.8 \mathrm{~Hz}$

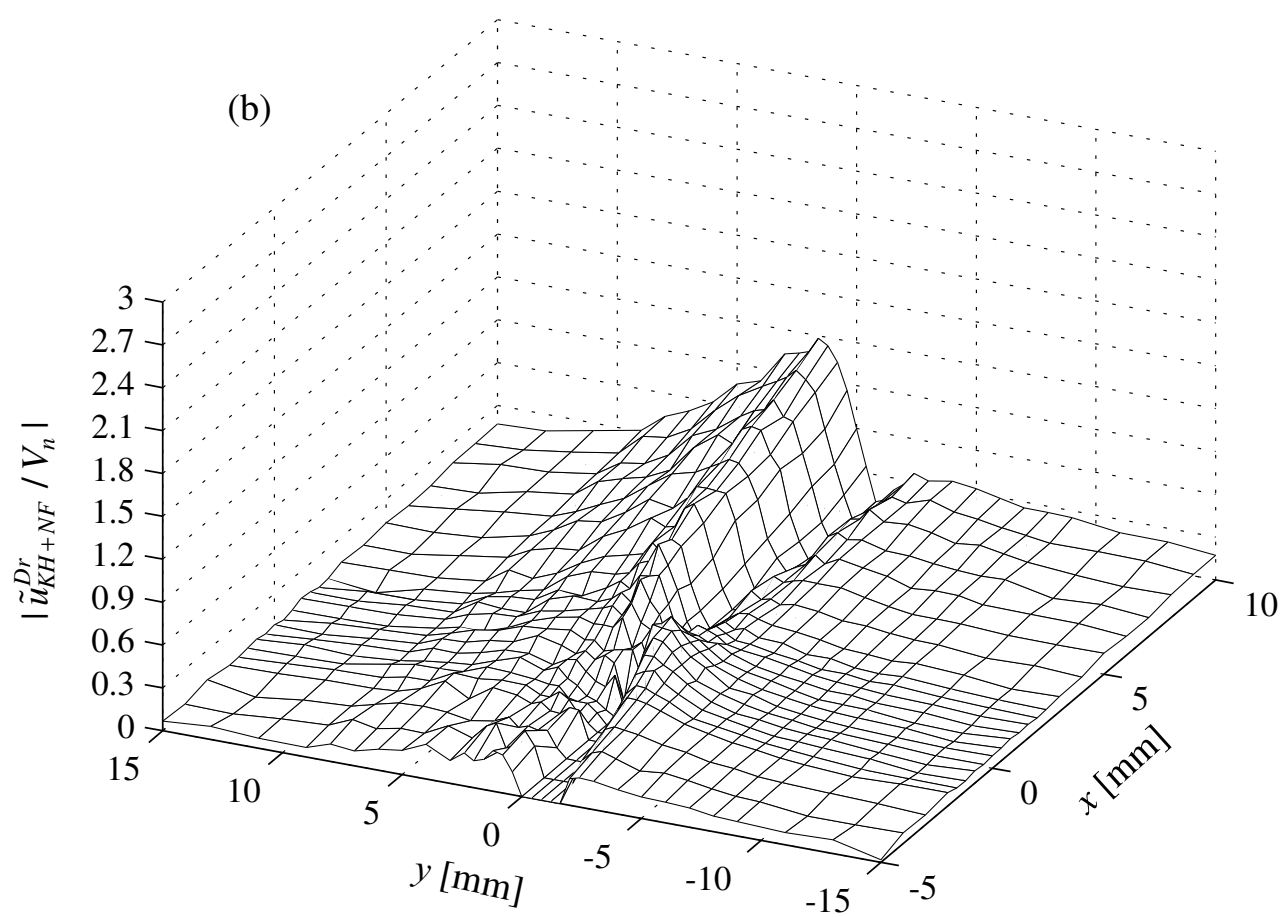

Abb. 4.9: Übersicht über den Betrag der normierten $\tilde{u}$-Komponente der Wechselgeschwindigkeit bei $0.8 \mathrm{~Hz}$ : (a) das Gesamtfeld $\tilde{u}_{A l l}^{D r}$; (b) $\tilde{u}_{K H+N F}^{D r}$ (das anregende Feld ist vom Gesamtfeld abgezogen) 


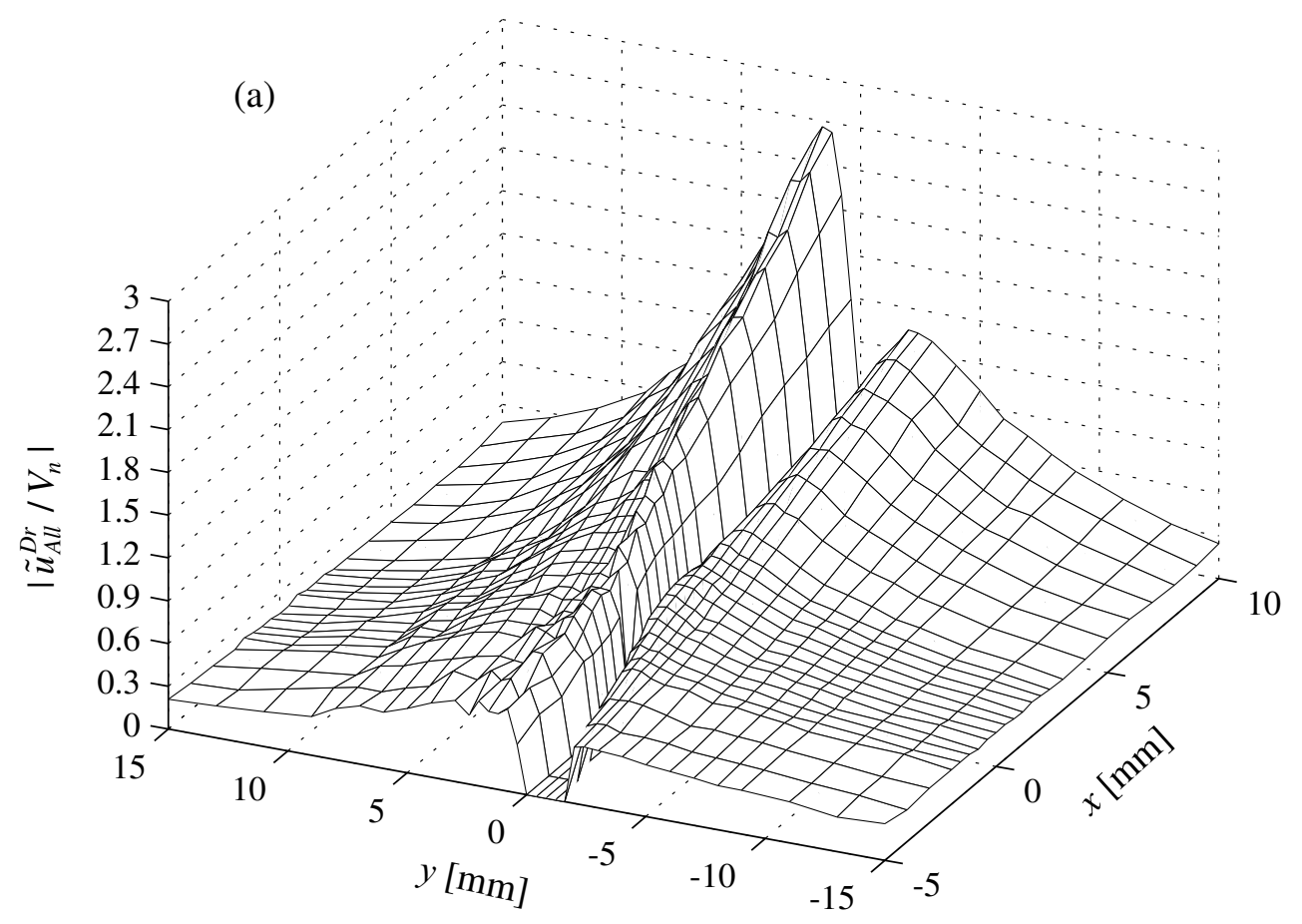

Anregungsfrequenz $=1.6 \mathrm{~Hz}$

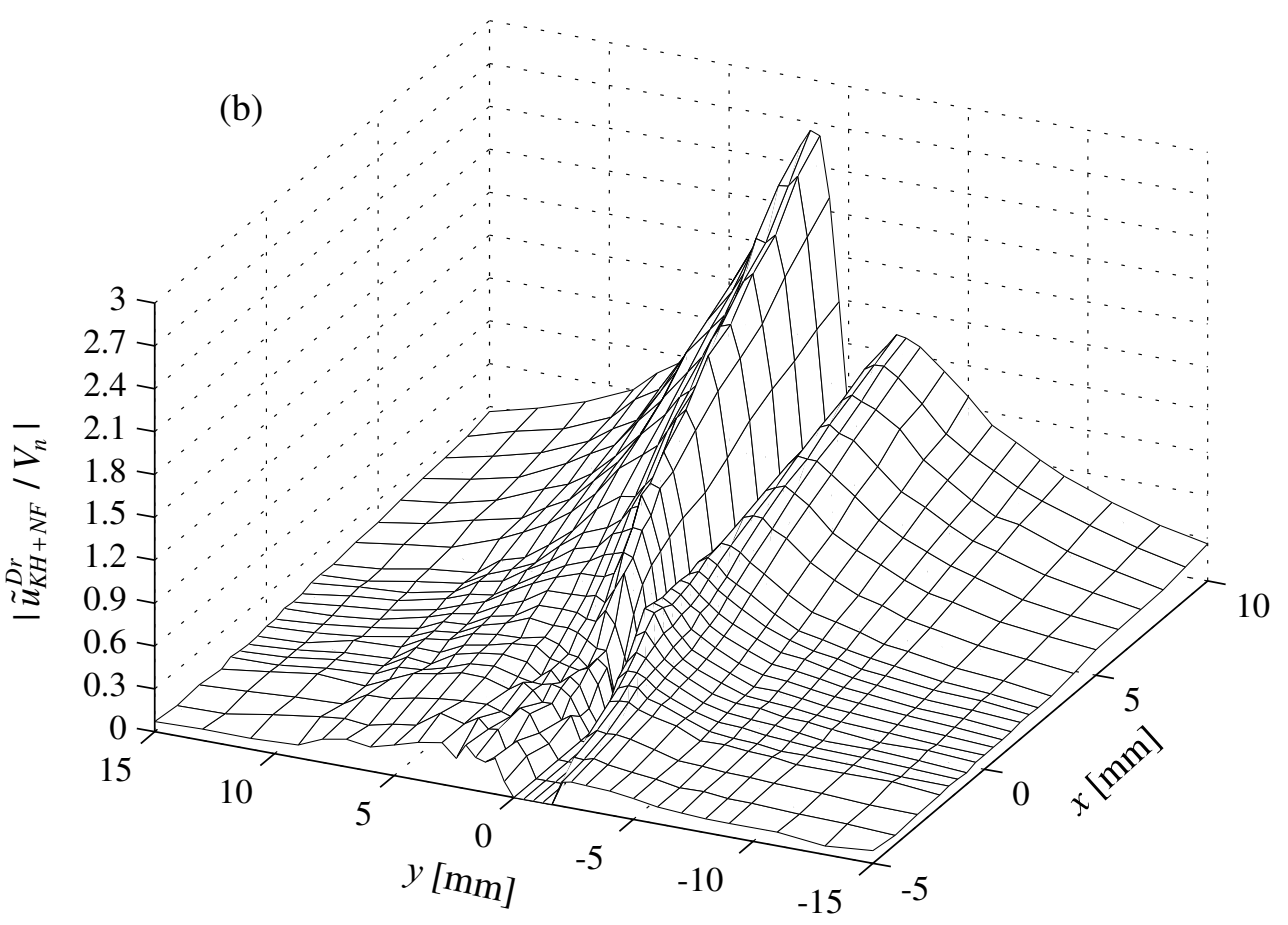

Abb. 4.10: Übersicht über den Betrag der normierten $\tilde{u}$-Komponente der Wechselgeschwindigkeit bei $1.6 \mathrm{~Hz}$ : (a) das Gesamtfeld $\tilde{u}_{A l l}^{D r}$; (b) $\tilde{u}_{K H+N F}^{D r}$ (das anregende Feld ist vom Gesamtfeld abgezogen) 


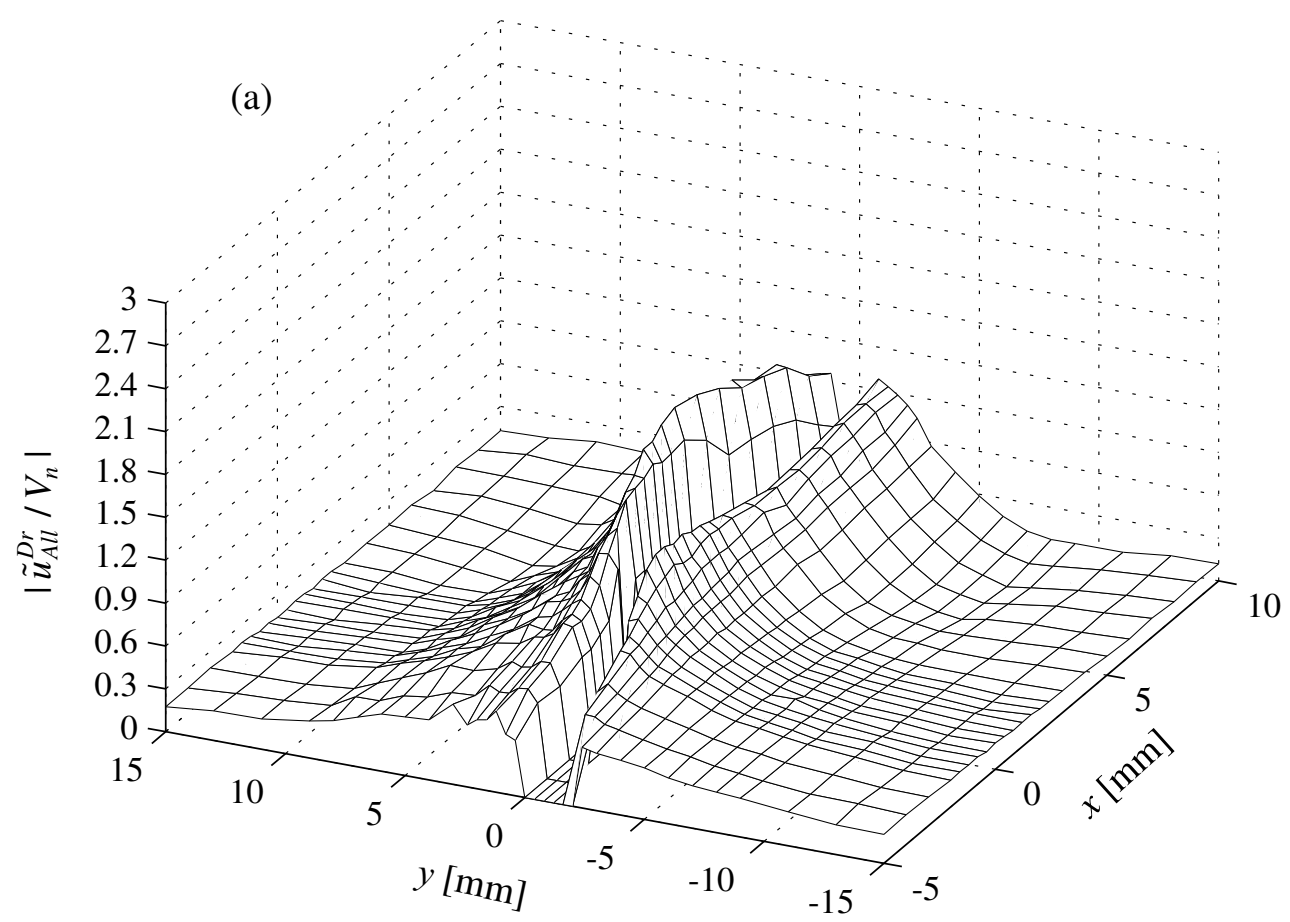

Anregungsfrequenz $=2.4 \mathrm{~Hz}$

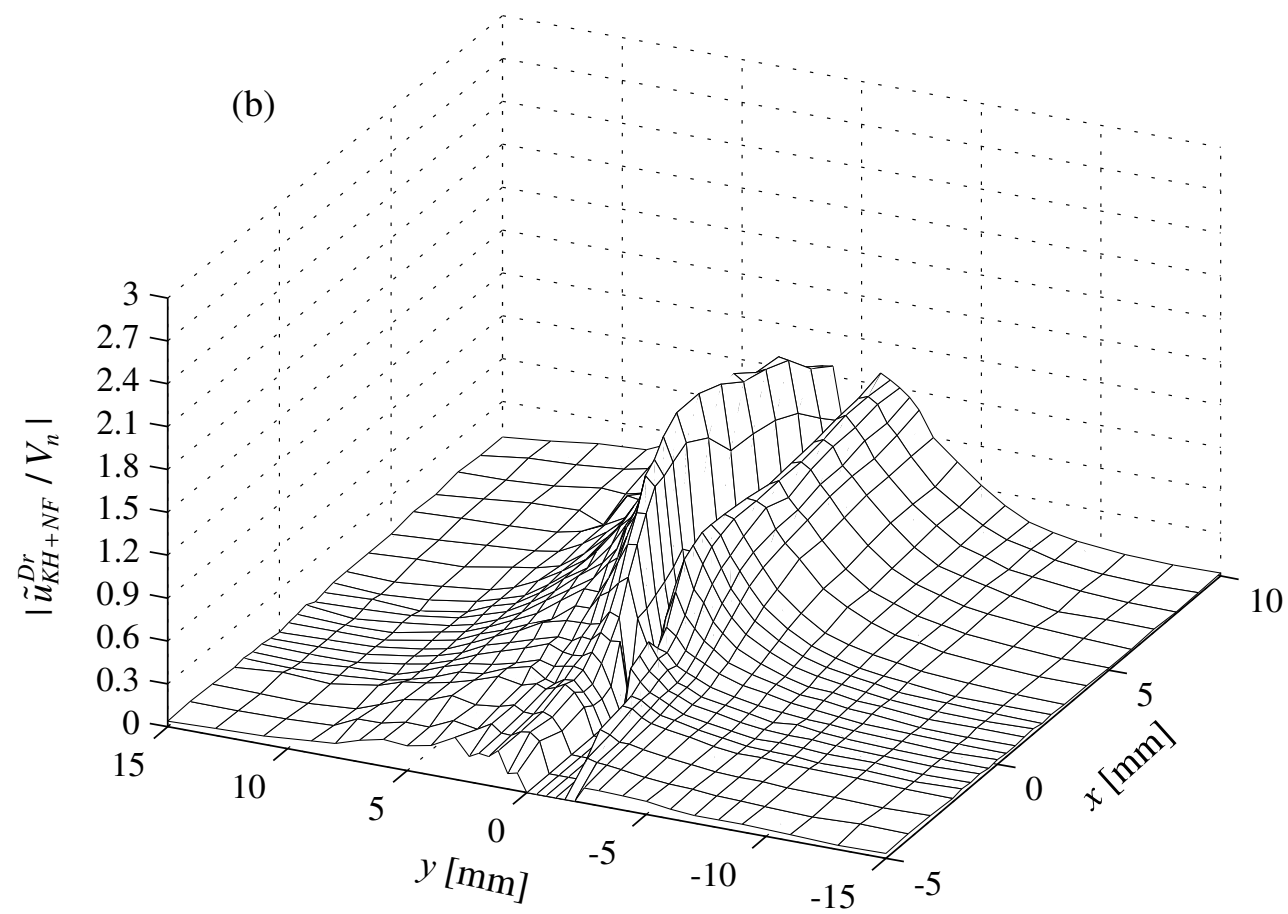

Abb. 4.11: Übersicht über den Betrag der normierten $\tilde{u}$-Komponente der Wechselgeschwindigkeit bei $2.4 \mathrm{~Hz}$ : (a) das Gesamtfeld $\tilde{u}_{A l l}^{D r}$; (b) $\tilde{u}_{K H+N F}^{D r}$ (das anregende Feld ist vom Gesamtfeld abgezogen) 
Maximum im Nebenströmungsbereich, das bei $0.8 \mathrm{~Hz}$ nicht in $\tilde{u}_{A l l}^{D r}$ zu sehen war. Der "Peak" ist erwartungsgemäß auch bei $\tilde{u}_{K H+N F}^{D r}$ wieder zu finden, befindet sich aber nur auf dem Rücken des Nebenströmungsbereichs, nicht jedoch auf dem Rücken des Hauptströmungsbereichs.

Bei $2.4 \mathrm{~Hz}$ stellt man fest, dass die Wechselwirkung zwischen ablösender Scherschicht und anregendem Feld mit abnehmender Wellenlänge der Instabilitätswelle komplexer geworden ist, da eine systematische exponentielle Entwicklung von sowohl $\tilde{u}_{A l l}^{D r}$ als auch $\tilde{u}_{K H+N F}^{D r}$ in $x$-Richtung für diese Strouhalzahl im Hauptströmungsbereich nicht zu erkennen ist, obwohl man die Ausbreitung der Instabilitätswellen bei $\tilde{v}_{K H+N F}^{D r}$ (Abbildung 4.11) beobachten konnte. Die Interferenz zwischen $\tilde{v}_{K H}^{D r}$ und $\tilde{v}_{A n F}^{D r}$, die bei $\tilde{u}_{A l l}^{D r}$ im Nebenströmungsbereich vorhanden ist, ist bei dem Feld $\tilde{u}_{K H+N F}^{D r}$ allerdings verschwunden (im Hauptströmungsbereich ist sie bei dieser Darstellung nicht erkennbar).

Es stellt sich jetzt die Frage, ob es noch ein zusätzliches Feld besonders im Hauptströmungsbereich gibt, das bei der Zerlegung des Messfeldes (Gleichung (4.5)) nicht berücksichtigt wurde, oder ob es sein kann, dass das Nahfeld $\tilde{u}_{N F}^{D r}$ so groß ist, dass das exponentiell anwachsende KH-Wellenfeld im Hauptströmungsbereich vollkommen überdeckt wird, so dass in dem hier betrachteten Messfeld nur die Abnahme von $\tilde{u}_{K H+N F}^{D r}$ mit der Entfernung von der Hinterkante zu beobachten ist. Es ist allerdings unwahrscheinlich, dass das Nahfeld $\tilde{u}_{N F}^{D r}$ so groß wird, da sonst die zugehörige $\tilde{v}$-Komponente $\tilde{v}_{N F}^{D r}$ auch entsprechend groß sein müsste, was aber in Abbildung 4.8 (b) nicht erkennbar war: Das Phänomen der Betragsabnahme in der Scherschicht ist nur bei der $\tilde{u}$ Komponente besonders auf der Hauptströmungsseite zu beobachten.

Hier wird nun ein Gedankenexperiment durchgefürt, um dieses Phänomen zu verstehen. Denkt man an das Gleichströmungsprofil $\bar{u}(y)$ in der Scherschicht, so fällt auf, dass sich der größte Teil des Geschwindigkeitsgradienten $\partial \bar{u}(y) / d y$ im Hauptströmungsbereich befindet (siehe Abbildung 3.3). Da die Scherschicht mit einer Auslenkung $\tilde{\eta}$ aufgrund der sinusförmigen Störungen durch den Druckgradienten schwingt, verlagert sich im einfachen Fall auch das Profil $\bar{u}(y)$ periodisch. Es ist daher anzunehmen, dass ein Teil der Wechselgeschwindigkeit von $\tilde{u}$ durch diese periodische Verschiebung des Gleichströmunpsprofils entsteht.

Hätte man ein anregendes Feld, das Störungen idealerweise nur senkrecht zur Scherschicht mit einer räumlich konstanten Geschwindigkeit $\tilde{v}_{A n F}$ bewirkt, so würde man durch die Wechselwirkung zwischen einer Scherströmung $\bar{u}(x, y), \bar{v}(x, y)$ und diesem anregenden Feld $\tilde{v}_{A n F}$ eine scheinbare Wechselgeschwindigkeit $\tilde{u}_{s}$ der $\tilde{u}$-Komponente erhalten, wobei $\tilde{u}_{A n F}=0$ angenommen wird und die Splitterplatte im Gedankenexperiment nicht vorhanden ist. Da in diesem Beispiel $\partial \tilde{p} / \partial x=0$ und $\partial \tilde{p} / \partial y$ räumlich konstant ist, ergibt sich für die scheinbare Geschwindigkeit $\tilde{u}_{s}$ durch die linearisierten Euler-Gleichung:

$$
\frac{\partial u_{s}}{\partial t}+\tilde{v}_{A n F} \frac{\partial \bar{u}}{\partial y}=0 \quad \Rightarrow \quad \tilde{u}_{s}=-\tilde{\eta}_{A n F} \frac{\partial \bar{u}}{\partial y}, \quad \text { wobei } \quad \tilde{\eta}_{A n F}=\frac{\tilde{v}_{A n F}}{-i \beta}
$$

Verallgemeinert man das Modell und betrachtet nun die scheinbaren Wechselgeschwindigkeiten $\tilde{u}_{s}$ und $\tilde{v}_{s}$, die sich nicht nur aus dem durch $\partial \bar{u} / \partial y$ verursachten Term sondern auch durch $\partial \bar{u} / \partial x, \partial \bar{v} / \partial x$, sowie $\partial \bar{v} / \partial y$ mit den anregenden Feldern $\tilde{u}_{A n F}$ und $\tilde{v}_{A n F}$ ergeben und vollständig im folgenden beschrieben werden: 


$$
\tilde{u}_{s}=\frac{1}{i \beta}\left(\tilde{u}_{A n F} \frac{\partial \bar{u}}{\partial x}+\tilde{v}_{A n F} \frac{\partial \bar{u}}{\partial y}\right), \quad \tilde{v}_{s}=\frac{1}{i \beta}\left(\tilde{u}_{A n F} \frac{\partial \bar{v}}{\partial x}+\tilde{v}_{A n F} \frac{\partial \bar{v}}{\partial y}\right)
$$

wobei $\tilde{u}_{A n F}$ und $\tilde{v}_{A n F}$ räumlich konstant sind.

Man sieht bei diesem Gedankenexperiment, dass die Wechselgeschwindigkeiten $\tilde{u}_{s}$ und $\tilde{v}_{s}$ nur durch eine scheinbare Verschiebung des Koordinatensystems entstehen und nicht einmal die Instabilitätswellen durch ein konstantes anregendes Feld $\tilde{u}_{A n F}, \tilde{v}_{A n F}$ angeregt werden können. Das scheinbare Wechselgeschwindigkeitsfeld kann in diesem Beispiel mit einer Stromfunktion $\tilde{\psi}_{s}$ beschrieben werden:

$$
\tilde{\psi}_{s}=\left[\left(\frac{\tilde{u}_{A n F}}{i \beta}\right) \frac{\partial}{\partial x}+\left(\frac{\tilde{v}_{A n F}}{i \beta}\right) \frac{\partial}{\partial y}\right] \bar{\psi}, \quad \text { wobei } \quad \bar{u}=\frac{\partial \bar{\psi}}{\partial y}, \quad \bar{v}=-\frac{\partial \bar{\psi}}{\partial x}
$$

Bei der untersuchten Scherströmung ist die scheinbare Wechselgeschwindigkeit gemäß Gleichung (4.11) allerdings kein eigenständiges Feld, das z. B. die Kontinuitätsgleichung erfüllt, da das anregende Feld $\tilde{\psi}_{A n F}^{D r}$ bzw. $\tilde{\psi}_{A n F}^{K l}$ durch die Anwesenheit der Splitterplatte stark inhomogen ist. Nimmt man aber an, dass es sich bei dem scheinbaren Geschwindigkeitsfeld nicht um eine dynamische sondern eine kinematische Betrachtung handelt, so kann man näherungsweise die Gleichung (4.11) weiter verwenden. Man darf dann insbesondere nicht erwarten, dass die scheinbare Wechselgeschwindigkeit $\tilde{u}_{s}^{D r}$ und $\tilde{v}_{s}^{D r}$ die Kontinuitätsgleichung erfüllt (ebenso wie $\tilde{u}_{s}^{K l}$ und $\left.\tilde{v}_{s}^{K l}\right)$. Die Divergenz von $\left(\tilde{u}_{s}^{D r}, \tilde{v}_{s}^{D r}\right)^{T}$ lässt sich nun mit indizierten Geschwindigkeitskomponenten, d.h. $\tilde{u} \rightarrow \tilde{u}_{1}, \tilde{v} \rightarrow \tilde{u}_{2}$ und mit Hilfe der Gleichung (4.11) wie folgt schreiben:

$$
\sum_{j} \frac{\partial}{\partial x_{j}} \tilde{u}_{s, j}=\frac{1}{i \beta} \sum_{j, k}\left[\frac{\partial}{\partial x_{j}}\left(\tilde{u}_{A n F, k}^{D r} \frac{\partial \bar{u}_{j}}{\partial x_{k}}\right)\right]=\frac{1}{i \beta} \sum_{j, k}[\underbrace{\left.\frac{\partial \tilde{u}_{A n F, k}^{D r} \frac{\partial \bar{u}_{j}}{\partial x_{j}} \frac{\bar{u}_{k}}{\partial x_{k}}}{\tilde{u}_{A n F, k}^{D r} \frac{\partial^{2} \bar{u}_{j}}{\partial x_{j} \partial x_{k}}}\right]}_{\tilde{T}^{D r}} \underbrace{\underbrace{}_{0}}_{=0}
$$

Aufgrund des Terms $\tilde{T}^{D r}$ (bei Schwingkanten-Anregung wäre es $\tilde{T}^{K l}$ ) ist das scheinbare Geschwindigkeitsfeld nicht divergenzfrei, und man kann daher keine Stomfunktion für den scheinbaren Geschwindigkeitsanteil angeben. Diese Tatsache führt dazu, dass man kein in jeder Hinsicht sinnvolles Nahfeld erhält, wenn man die scheinbare Wechselgeschwindigkeit abzieht.

Trotzdem ist es interessant zu untersuchen, wie sich $\tilde{\psi}_{K H+N F}^{D r}$ verändert, wenn die scheinbaren Geschwindigkeiten abgezogen werden. Zur Darstellung des Wechselfeldes ohne scheinbare Geschwindigkeiten werden nun $\tilde{u}_{K H+N F-s}$ und $\tilde{v}_{K H+N F-s}$ mit Hilfe der Gleichung (4.11) eingeführt, die unter der Annahme $\bar{v} \approx 0$ folgendermaßen definiert sind:

$$
\tilde{u}_{K H+N F-s}=\tilde{u}_{K H+N F}-\tilde{u}_{s}, \quad \tilde{v}_{K H+N F-s}=\tilde{v}_{K H+N F}-\tilde{v}_{s} \approx \tilde{v}_{K H+N F}
$$

Abbildung 4.12 zeigt den Betrag der komplexen Amplitude $\tilde{u}_{K H+N F-s}$ für die drei Anregungsfrequenzen. Vergleicht man $\tilde{u}_{K H+N F}^{D r}$ mit $\tilde{u}_{K H+N F-s}^{D r}$, so erkennt man deutlich, dass die scheinbare Geschwindigkeit $\tilde{u}_{s}$ auch ein Teil der Wechselwirkung besonders im Kantenbereich ist, aber offensichtlich nicht zu dem Feld der Instabilitätswellen 
(a) Anregungsfrequenz

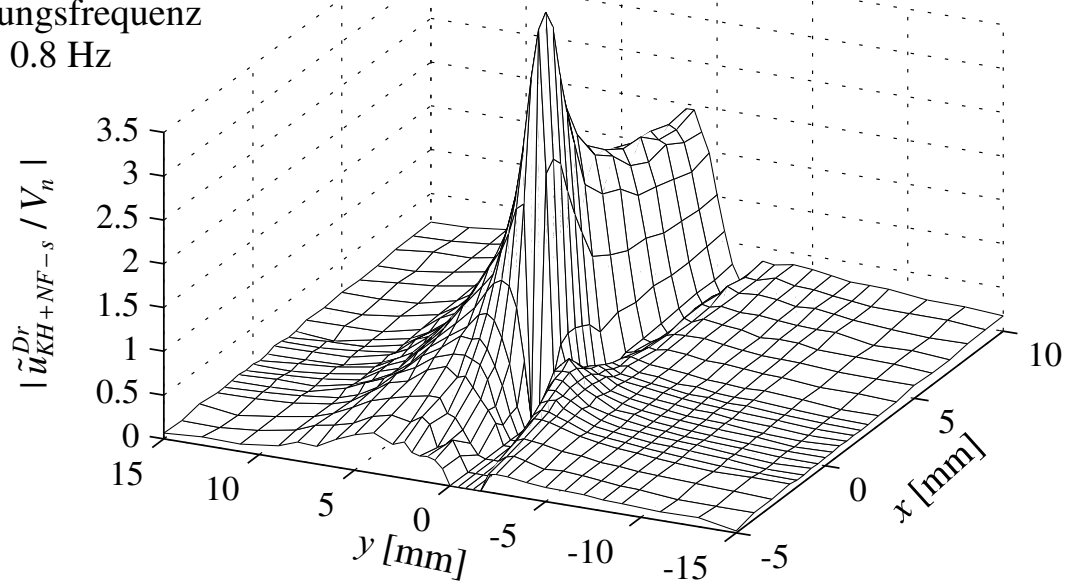

(b) Anregungsfrequenz $1.6 \mathrm{~Hz}$

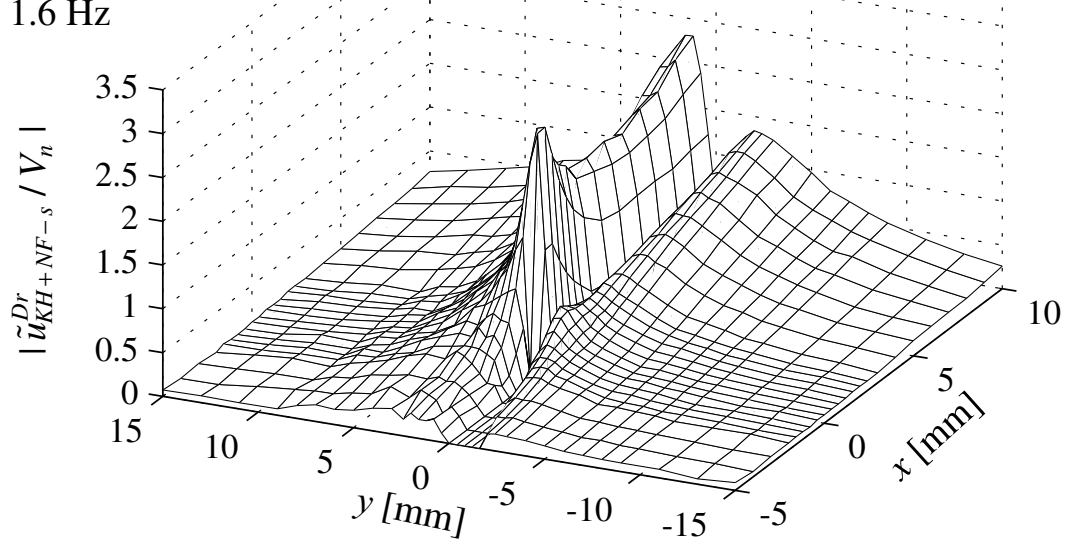

(c) Anregungsfrequenz

$2.4 \mathrm{~Hz}$

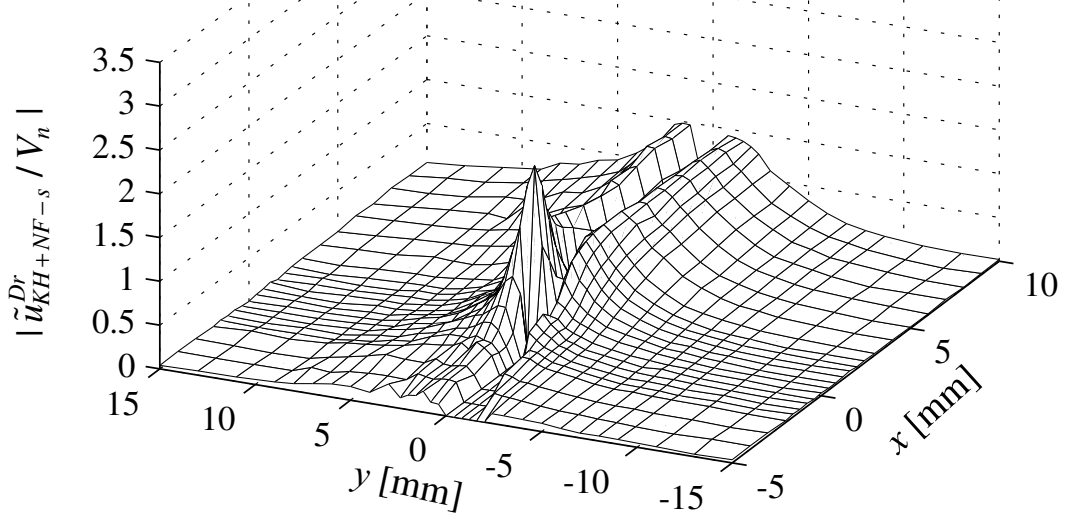

Abb. 4.12: Übersicht über den Betrag der normierten Wechselgeschwindigkeit $\tilde{u}_{K H+N F-s}$ ohne die scheinbare Geschwindigkeit $\tilde{u}_{s}$ bei (a) $0.8 \mathrm{~Hz}$, (b) $1.6 \mathrm{~Hz}$ und (c) $2.4 \mathrm{~Hz}$ 
gehört. Auffällig für alle drei Frequenzen ist, dass der „Peak“ unmittelbar an der Hinterkante im Hauptströmungsbereich erscheint, während er bei $\tilde{u}_{K H+N F}^{D r}$ nur im Nebenströmungsbereich zu sehen ist. Bei $f=2.4 \mathrm{~Hz}$ ist nun zu beobachten, dass das Maximum des Betrages von $\tilde{u}_{K H+N F-s}^{D r}$ im Hauptströmungsbereich bei entsprechend geringer Anfachung mit steigender $x$-Koordinate anwächst, während der Betrag von $\tilde{u}_{K H+N F}^{D r}$ abnimmt.

Man gewinnt schließlich den Eindruck aus der Darstellung von $\tilde{u}_{K H+N F-s}^{D r}$, dass das Feld der Kelvin-Helmholtz- Instabilität $\tilde{u}_{K H}^{D r}$ besser bei $\tilde{u}_{K H+N F-s}^{D r}$ betrachtet werden kann als bei $\tilde{u}_{K H+N F}^{D r}$. Diese Tatsache könnte dazu führen, dass $\tilde{\psi}_{K H}^{D r}$ aus $\tilde{u}_{K H+N F-s}^{D r}$ und $\tilde{v}_{K H+N F-s}^{D r}$ genauer extrahiert werden kann als aus $\tilde{\psi}_{K H+N F}^{D r}$. Zur Verifizierung dieser Hypothese ist jedoch notwendig, dass das einzelne theoretische KH-Profil mit $\tilde{u}_{K H+N F-s}^{D r}$ und $v_{K H+N F-s}^{D r}$ quantitativ verglichen wird.

\subsubsection{Das Profil der Kelvin-Helmholtz-Instabilitätswellen}

Zur Bestimmung des Nahfeldes $\tilde{\psi}_{N F}$, das durch die Wechselwirkung zwischen der Gleichströmung und dem anregenden Feld erzeugt wird, ist eine möglichst genaue Beschreibung des Feldes der Kelvin-Helmholtz-Instabilitätswelle $\tilde{\psi}_{K H}$ notwendig. In diesem Abschnitt wird daher ein gemessenes Profil sowohl bezüglich der $\tilde{u}_{\text {- als }}$ auch der $\tilde{v}$-Komponente mit dem theoretischen Profil verglichen, das durch die Rayleigh-Gleichung bestimmt wird (siehe Abschnitt 4.1.1). Zum Vergleich zwischen Messergebnissen und theoretischen Profilen werden dabei nicht nur die angefachte, sondern auch die gedämpfte Lösung der KH-Wellen berücksichtigt. Der in Gleichung (4.7) dargestellte Ansatz zeigt, dass man zur Anpassung zwei freie komplexe Amplituden $C_{A}$ und $C_{D}$ benötigt.

$\underline{\text { Anpassung des KH-Profils an } \tilde{u}_{K H+N F} \text { und } \tilde{v}_{K H+N F} \text { bzw. an } \tilde{u}_{K H+N F-s} \text { und } \tilde{v}_{K H+N F-s}}$

In der Diskussion des vorigen Abschnitts hat sich die Frage gestellt, ob $\tilde{u}_{K H+N F-s}$ und $\tilde{v}_{K H+N F-s}$ besser zum Vergleich mit den theoretischen KH-Wellen geeignet sind als das Feld inklusive der scheinbaren Wechselgeschwindigkeit. Die beiden Felder werden daher daraufhin untersucht, welches sich besser durch die Überlagerung einer angefachten und einer gedämpften KH-Welle beschreiben lässt. Die Abbildungen 4.13 (a) und (c) zeigen den Vergleich der Messwerte $\tilde{u}_{K H+N F}^{D r}$ und $\tilde{v}_{K H+N F}^{D r}$ mit den angepassten theoretischen Kurven $\tilde{u}_{K H}^{D r}$ bzw. $\tilde{v}_{K H}^{D r}$ für Betrag und Phase, während die theoretischen Kurven in Abbildungen 4.13 (b) und (d) mit $\tilde{u}_{K H+N F-s}$ und $\tilde{v}_{K H+N F-s}$ verglichen werden. Die Anregungsfrequenz ist dabei $2.4 \mathrm{~Hz}$ und die Messungen sind in der Scherschicht bei $x=10 \mathrm{~mm}$ durchgeführt worden, da man erwarten kann, dass dort das Wechselfeld praktisch nur von den KH-Wellen dominiert wird. Zwei freie Parameter $C_{A}$ und $C_{D}$ wurden durch Anpassung so bestimmt, dass das Quadrat der Abweichung zwischen den Mess- und den Theoriewerten sowohl für die $\tilde{u}$ - als auch für die $\tilde{v}$-Komponente auf der $y$-Koordinate minimal wird.

Obwohl der Verlauf der theoretischen Kurven im wesentlichen mit den Messwerten von $\tilde{u}_{K H+N F}^{D r}$ und $\tilde{v}_{K H+N F}^{D r}$ übereinstimmt, geben der Verlauf von $\tilde{u}_{K H+N F-s}^{D r}$ und $\tilde{v}_{K H+N F-s}^{D r}$ die 
Theoriekurven noch deutlich besser wieder und zwar sowohl für die $\tilde{u}$-als auch für die $\tilde{v}$-Komponente. Den Unterschied zwischen Messwerten und Theoriekurven erkennt man sowohl im Betrag als auch in der Phase, insbesondere im Hauptströmungsbereich der $\tilde{u}$-Komponete, da dort die scheinbare Geschwindigkeit $\tilde{u}_{s}$ große Werte annimmt.

An dieser Auftragung wird offensichtlich, dass die scheinbare Geschwindigkeit die Anpassung des KH-Profils an das Messfeld tatsächlich stört und ohne Berücksichtigung von $\tilde{u}_{s}$ die zwei freien Parameter $C_{A}$ und $C_{D}$ ungenauer abgeschätzt werden können. Da das Feld von $\tilde{u}_{s}$ und $\tilde{v}_{s}$ aus Gleichung (4.13) nicht divergenzfrei ist, kann man es nicht ohne weiteres zur Bestimmung des Nahfeldes $\tilde{\psi}_{N F}$ verwenden. Andererseits ist es aber sinnvoll $\tilde{u}_{K H+N F-s}$ und $\tilde{v}_{K H+N F-s}$ für die Ermittlung des KH-Wellenfeldes $\tilde{\psi}_{K H}$.

Eine interessante Frage ist nun, wie groß der verbleibende Term $\tilde{T}^{D r}$ von Gleichung (4.13) ist, der durch Wechselwirkung zwischen den Gradienten der Gleichströmung und dem anregenden Feld zustande kommt. Da die in den Abbildungen 4.13 (b) und (d) dargestellten Größen $\tilde{u}_{K H+N F-s}^{D r}$ und $\tilde{v}_{K H+N F-s}^{D r}$ nahezu exakt von dem theoretischen bestimmten Feld $\tilde{\psi}_{K H}^{D r}$ beschrieben werden, kann man davon ausgehen, dass an der Stelle $x=10 \mathrm{~mm} \tilde{\psi}_{K H+N F-s}^{D r}=\tilde{\psi}_{K H}^{D r}$. Der Term $\tilde{T}^{D r}$ spielt dort also keine Rolle.

$\underline{\text { Profile der Kelvin-Helmholtz-Wellen für verschiedene Strouhalzahlen }}$

Aus der durch die Rayleigh-Gleichung bestimmten Dispersionsrelation erhält man das KH-Wellenprofil, das neben dem Gleichströmungsprofil von der Strouhalzahl abhängig ist. Die durch den Druckgradienten angeregten Wechselgeschwindigkeiten wurden nun bei einem Abstand von $x=10 \mathrm{~mm}$ stromab der Ablösekante für verschiedene Anregungsfrequenzen aufgenommen und werden hier mit theoretischen Kelvin-HelmholtzWellenprofilen verglichen.

Aufgrund der obigen Diskussion wird im folgenden angenommen, dass $\tilde{u}_{K H+N F-s}$ und $\tilde{v}_{K H+N F-s}$ zur Anpassung des theoretischen KH-Profils mit Hilfe des Ansatzes von Gleichung (4.7) verwendet werden können und dass dabei die Verfälschung des Ergebnisses durch die scheinbaren Wechselgeschwindigkeiten $\tilde{u}_{s}$ und $\tilde{v}_{s}$ vermieden wird.

Die Abbildungen 4.14 und 4.15 zeigen den Vergleich zwischen Messwerten und Theoriekurven für die Beträge und Phasen der $\tilde{u}$ - bzw. $\tilde{v}$-Komponente. Die Anregungsfrequenzen sind dabei von oben nach unten (a) $0.8 \mathrm{~Hz}$, (b) $1.2 \mathrm{~Hz}$, (c) $1.6 \mathrm{~Hz}$ sowie (d) $2.0 \mathrm{~Hz}$ und der Vergleich bei $2.4 \mathrm{~Hz}$ ist in den Abbildungen 4.13 (b) und (d) zu sehen. Generell geben die theoretischen Kurven den Verlauf der Messwerte für alle Frequenzen sowohl für den Betrag als auch die Phase sehr gut wieder. Es lässt sich daher annehmen, dass das gemessene Wechselströmungsfeld an der Stelle $x=10 \mathrm{~mm}$ im Frequenzbereich $0.8 \mathrm{~Hz} \leq f \leq 2.4 \mathrm{~Hz}$ nur durch die angefachte und die gedämpfte Lösung der RayleighGleichung beschrieben wird. Bei $0.8 \mathrm{~Hz}$ erkennt man allerdings Abweichungen für die $\tilde{u}$-Komponente besonders im Nebenströmungsbereich. Da die Beträge der Messwerte in diesem Bereich systematisch unter der Theoriekurve liegen und der Phasenunterschied zwischen Mess- und Theoriewerten etwa konstant ist, ist anzunehmen, dass dem Messfeld ein Potenzialfeld überlagert ist. Aber da sich eine ähnliche Abweichung auch für $f=1.2 \mathrm{~Hz}$ ergibt, aber nicht mehr für höhere Frequenzen, lässt sich vermuten, dass das Potenzialfeld eventuell zu dem Nahfeld $\tilde{\psi}_{N F}^{D r}$ gehört. Bei tiefen Anregungsfrequen- 

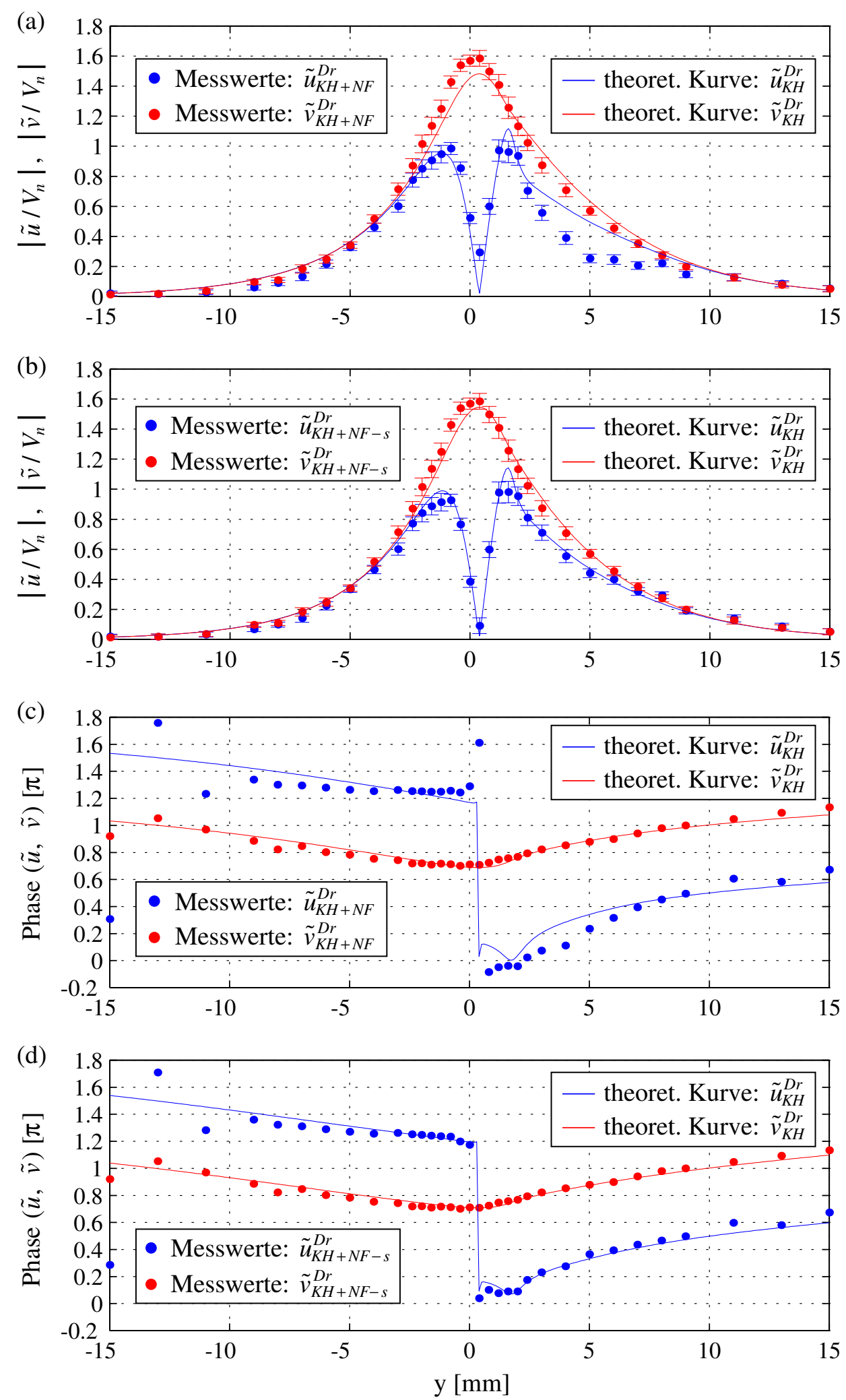

Abb. 4.13: Vergleich zwischen gemessenen und theoretischen KH-Wellenprofilen $\tilde{u}_{K H}^{D r}$, $\tilde{v}_{K H}^{D r}$ in der Scherschicht bei $x=10 \mathrm{~mm}$ für $2.4 \mathrm{~Hz}$ : (a) Vergleich der Beträge zwischen $\tilde{u}_{K H+N F}^{D r}$ und $\tilde{u}_{K H}^{D r}$ sowie $\tilde{v}_{K H+N F}^{D r}$ und $\tilde{v}_{K H}^{D r}$ (b) Vergleich zwischen $\left|\tilde{u}_{K H+N F-s}^{D r}\right|$ und $\left|\tilde{u}_{K H}^{D r}\right|$ sowie $\left|\tilde{v}_{K H+N F-s}^{D r}\right|$ und $\left|\tilde{v}_{K H}^{D r}\right|$ (c) Vergleich der Phasen von $\tilde{u}_{K H+N F}^{D r}$ und $\tilde{u}_{K H}^{D r}$ sowie von $\tilde{v}_{K H+N F}^{D r}$ und $\tilde{v}_{K H}^{D r}(\mathrm{~d})$ Vergleich der Phasen von $\tilde{u}_{K H+N F-s}^{D r}$ und $\tilde{u}_{K H}^{D r}$ sowie von $\tilde{v}_{K H+N F-s}^{D r}$ und $\tilde{v}_{K H}^{D r}$ 

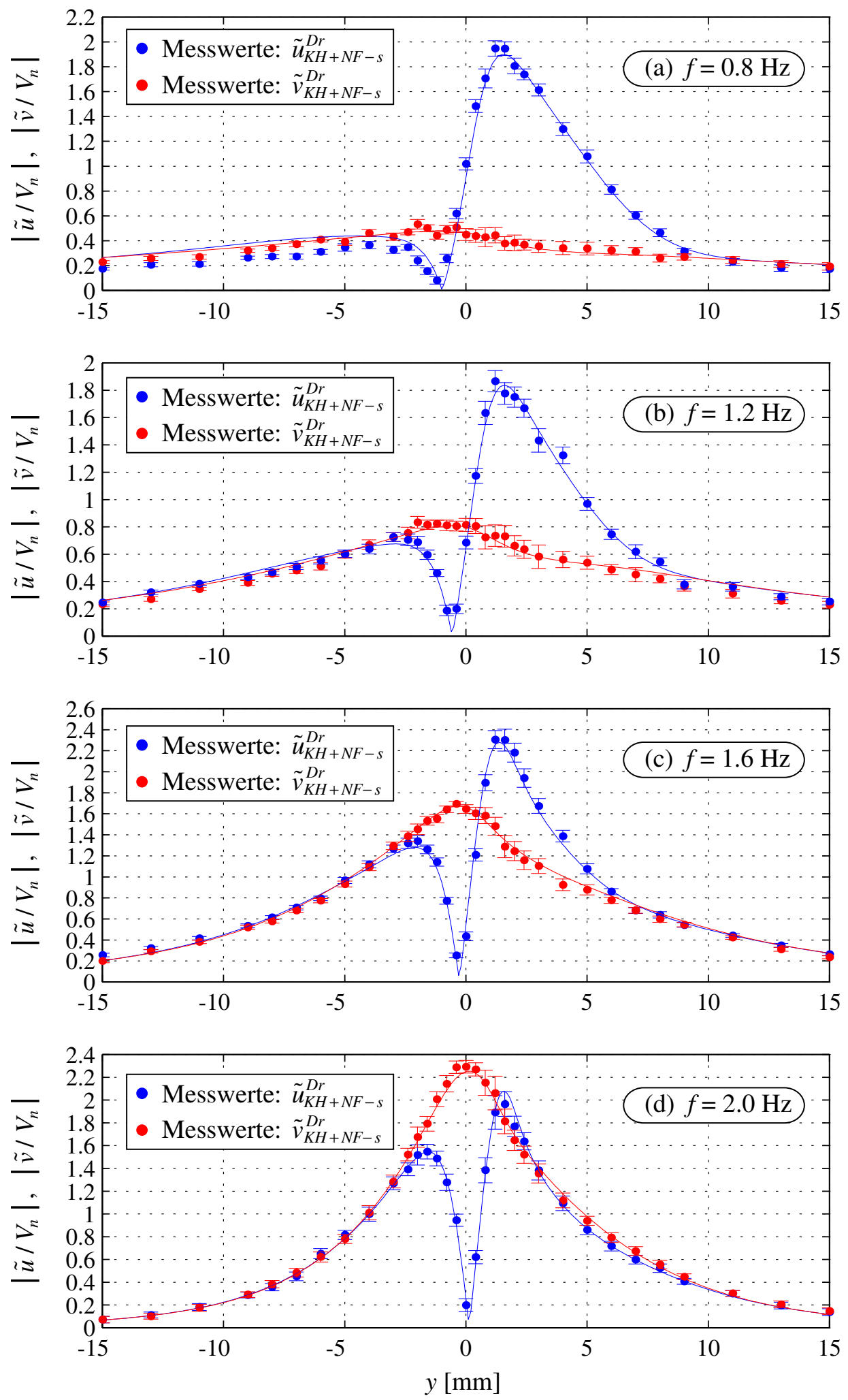

Abb. 4.14: Vergleich der Messwerte $\left|\tilde{u}_{K H+N F-s}^{D r}\right|,\left|\tilde{v}_{K H+N F-s}^{D r}\right|$ mit den durch die RayleighGleichung bestimmten Theoriekurven $\left|\tilde{u}_{K H}^{D r}\right|,\left|\tilde{v}_{K H}^{D r}\right|$ in der Scherschicht bei $x=10 \mathrm{~mm}$ : (a) $f=0.8 \mathrm{~Hz},(\mathrm{~b}) f=1.2 \mathrm{~Hz}$, (c) $f=1.6 \mathrm{~Hz}$ und (d) $f=2.0 \mathrm{~Hz}$ 

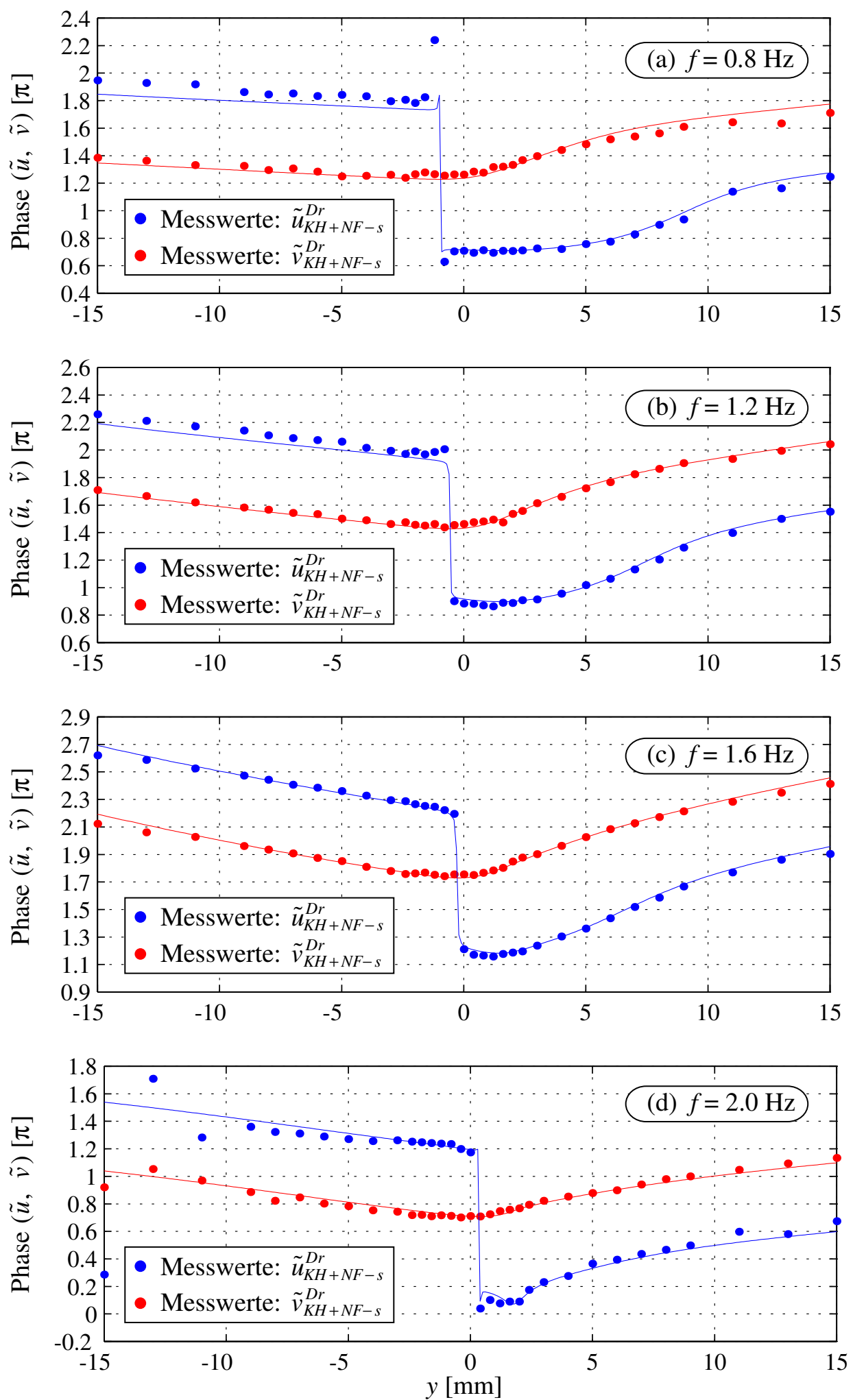

Abb. 4.15: Vergleich der Phasen von $\tilde{u}_{K H+N F-s}^{D r}$ und $\tilde{v}_{K H+N F-s}^{D r}$ mit den aus der RayleighGleichung resultierenden $\arg \left\{\tilde{u}_{K H}^{D r}\right\}$ und $\arg \left\{\tilde{v}_{K H}^{D r}\right\}$ in der Scherschicht bei $x=10 \mathrm{~mm}$ : (a) $f=0.8 \mathrm{~Hz}$, (b) $f=1.2 \mathrm{~Hz}$, (c) $f=1.6 \mathrm{~Hz}$ und (d) $f=2.0 \mathrm{~Hz}$ 
zen ist die Wellenlänge der Instabilitätswellen noch lang, so dass sich die KH-Wellen bei $x=10 \mathrm{~mm}$ möglicherweise noch nicht vollständig ausgebildet haben. Das Nahfeld ist dann in diesem Messbereich für tiefe Frequenzen nicht vernachlässigbar.

Da im Bereich von etwa $y<-8 \mathrm{~mm}$ und $y>11 \mathrm{~mm}$ die Gleichströmung wirbelfrei ist, muss in diesem Potenzialbereich $\nabla^{2} \tilde{\psi}_{K H}=0$ erfüllt sein. Berücksichtigt man dazu die Randbedingung der Rayleich-Gleichung $\tilde{\psi}_{K H}(x, y) \rightarrow 0$ für $y \rightarrow \pm \infty$, so ergibt sich die folgende einfache Beziehung zwischen $\tilde{u}_{K H}$ und $\tilde{v}_{K H}$ im Potenzialbereich

$$
\begin{cases}\tilde{u}_{K H}=-i \tilde{v}_{K H} & \text { für } y>0 \mathrm{im} \text { Potenzialbereich } \\ \tilde{u}_{K H}=i \tilde{v}_{K H} & \text { für } y<0 \mathrm{im} \text { Potenzialbereich }\end{cases}
$$

Bemerkenswert dabei ist, dass diese Beziehung unabhängig von den Parametern $C_{A}$ und $C_{D}$ ist, die der Gewichtung der angefachten bzw. gedämpften Lösung der KH-Wellen entsprechen. Aus der Auftragung in Abbildung 4.14 und 4.15 ist daher zu erkennen, dass die theoretischen Geschwindigkeiten $\left|\tilde{u}_{K H}\right|$ und $\left|\tilde{v}_{K H}\right|$ im Potenzialbereich für alle Strouhalzahlen gleiche Werte annehmen, und die Phase von $\tilde{u}_{K H}$ der Phase von $\tilde{v}_{K H}$ im Hauptströmungsbereich um $0.5 \pi$ nacheilt, während sie im Nebenströmungsbereich um $0.5 \pi$ vorauseilt. In der Scherschicht dagegen, wo Gleichwirbeligkeit vorhanden ist, ist die Beziehung zwischen $\tilde{u}_{K H}$ und $\tilde{v}_{K H}$ komplizierter, da in der oben beschriebenen Laplace-Gleichung die Stromfunktion $\tilde{\psi}_{K H}$ einen Quellenterm bekommt. Man erhält somit in der Scherschicht eine Poisson-Gleichung:

$$
\nabla^{2} \tilde{\psi}_{K H}=-\tilde{\omega}_{K H}, \quad \text { wobei } \quad \tilde{\omega}_{K H}=\frac{\partial \tilde{v}_{K H}}{\partial x}-\frac{\partial \tilde{u}_{K H}}{\partial y}
$$

In diesem Gebiet, wo die Wechselwirbeligkeit $\tilde{\omega}_{K H}$ entsteht, wie Grützmacher es in seiner Arbeit betrachtet hat (GRÜTZMACHER [14]), findet ein Energieaustausch zwischen Gleich- und Wechselströmung statt. Für eine homogene Scherschicht mit $u=\bar{u}(y)+\tilde{u}(x, y, t), v=\tilde{v}(x, y, t)$ wird dabei die kinetische Energie pro Masseneinheit definiert als

$$
E=\frac{1}{2}\left(u^{2}+v^{2}\right)=\frac{1}{2} \bar{u}^{2}+\bar{u} \tilde{u}+\frac{1}{2}\left(\tilde{u}^{2}+\tilde{v}^{2}\right) .
$$

Der dritte Term auf der rechten Seite stellt nun die Energie der Wechselströmung dar, während der erste Term der Energie der Gleichströmung entspricht, und der zweite Term, der durch die periodische Störung verursacht wird, im zeitlichen Mittel Null ist. Der zeitliche Mittelwert des dritten Terms ist schließlich gegeben durch

$$
\tilde{e}^{(2)}=\frac{1}{2} \overline{\left(\tilde{u}^{2}+\tilde{v}^{2}\right)}
$$

wobei der obere Index (2) von $\tilde{e}$ die zweite Ordnung bedeutet. Mit Hilfe der linearisierten Euler-Gleichung erhält man dann die folgende Gleichung für die Leistung:

$$
\frac{D \tilde{e}^{(2)}}{D t}=\frac{\partial \tilde{e}^{(2)}}{\partial t}+\bar{u} \frac{\partial \tilde{e}^{(2)}}{\partial x}=-\overline{\tilde{u} \tilde{v}} \frac{d \bar{u}}{d y}-\overline{\tilde{u} \frac{\partial \tilde{p}}{\partial x}}-\overline{\tilde{v} \frac{\partial \tilde{p}}{\partial y}}+\mathcal{O}(3)
$$



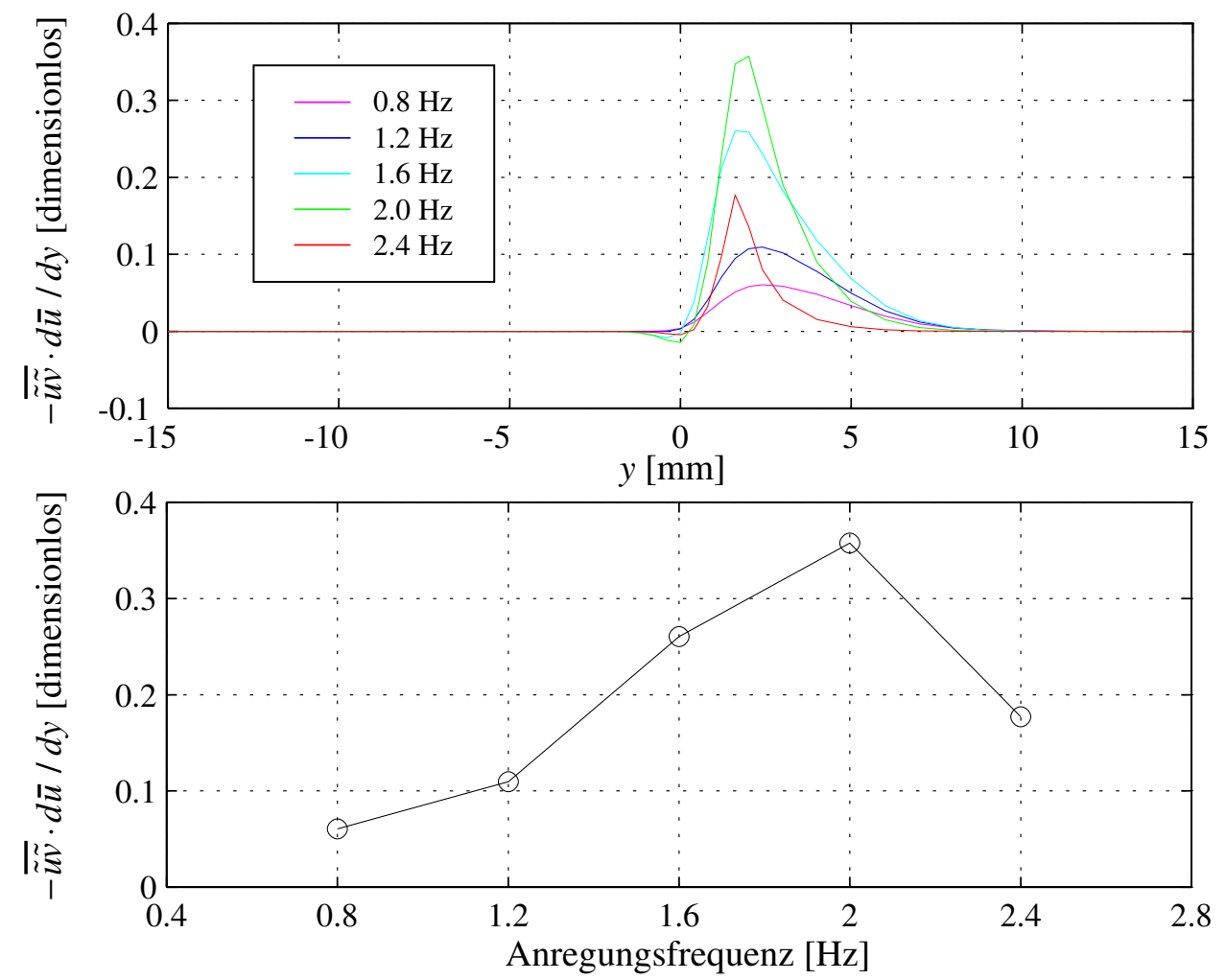

Abb. 4.16: Energietransfer zwischen Gleich- und Wechselströmung bei $x=10 \mathrm{~mm}$ für verschiedene Frequenzen bei Druckgradienten-Anregung: Abhängigkeit von der $y$ Koordinate (a) und von der Anregungsfrequenz (b)

Der Energieaustausch zwischen Gleich- und Wechselströmung wird dabei durch den ersten Term $-\overline{\tilde{u}} \tilde{v} d \bar{u} / d y$ beschrieben, während die letzten zwei Terme den Verlust (Gewinn) an kinetischer Energie bei einem Übergang zwischen Bereichen mit höherem (niedrigerem) Druck darstellen: Bei positivem Vorzeichen findet ein Energietransfer von der Gleich- in die Wechselströmung statt, bei negativem Vorzeichnen ist es umgekehrt.

In Abbildung 4.16 (a) ist dieser Energietransfer im Fall der angefachten Lösung der Rayleigh-Gleichung bei Druckgradienten-Anregung für die fünf verschiedenen MessFrequenzen bei $x=10 \mathrm{~mm}$ in Abhängigkeit von der $y$-Koordinate gezeigt, wobei der Energietransfer mit $V_{n}$, der Hauptströmungsgeschwindigkeit $U_{1}$ und der Impulsverlustdicke $\Theta_{0}$ dimensionlos gemacht ist. Wie erwartet geht Energie bei der angefachten KH-Welle von der Gleich- in die Wechselströmung über, wo $\tilde{u}$ und $\tilde{v}$ negativ korreliert sind. Bei der gedämpften Mode ist dagegen diese Richtung umgekehrt, da zwischen der angefachten und der gedämpften Lösung aus Gleichung (4.2) sowie (4.7) die folgende Beziehung für die Geschwindigkeitskomponenten gilt:

$$
\tilde{u}_{K H_{D}}=\tilde{u}_{K H_{A}}^{*}, \quad \tilde{v}_{K H_{D}}=-\tilde{v}_{K H_{A}}^{*},
$$

wobei der untere Index $K H_{A}$ bzw. $K H_{D}$ jeweils der angefachten bzw. gedämpften Mode der KH-Wellen entspricht. Der Bereich, in dem der Energieaustausch stattfindet, ist 
von der Strouhalzahl abhängig und ist bei tiefen Frequenzen breiter als bei höheren. Man gewinnt somit den Eindruck, dass der Bereich, wo $\nabla^{2} \tilde{\psi}_{K H}$ den Quellenterm $-\tilde{\omega}_{K H}$ erhält, mit steigender Anregungsfrequenz kleiner wird.

Abbildung 4.16 (b) zeigt die Abhängigkeit des Energiaustausches $\left(y=y_{\max }\right)$ von der Anregungsfrequenz. Man erkennt aus der Auftragung, dass die Struktur einer typischen Kurve für die Anfachungskonstante entspricht und der Energieaustausch bei $x=10 \mathrm{~mm}$ im Frequenzbereich etwa von $1.6 \mathrm{~Hz}$ bis $2.0 \mathrm{~Hz}$ am größten ist.

\subsubsection{Berücksichtigung der $x$-Abhängigkeit des Gleich- strömungsfeldes}

Die in Abschnitt 4.1.1 gestellte Frage war, wo genau die Instabiltätswellen anfangen sich stromab auszubreiten. Mit anderen Worten, ob man tatsächlich die Felder $\tilde{\psi}_{N F}$ und $\tilde{\psi}_{K H}$ auseinander halten und dann einen Keim im Nahfeld finden kann, der für die Anregung der Kelvin-Helmholtz-Instabilitätswellen verantwortlich ist. Zur Zerlegung dieser Felder sollte der räumliche Verlauf der KH-Wellen von der Hinterkante der Splitterplatte möglichst gut beschrieben werden. Obwohl die theoretischen KH-Wellenprofile, die durch die Rayleigh-Gleichung bestimmt wurden, die Messwerte bei $x=10 \mathrm{~mm}$ sehr gut wiedergegeben haben, kann man nicht erwarten, dass die theoretische Lösung das durch periodische Störungen angeregte Feld $\tilde{\psi}_{K H}$ vollständig beschreibt, da der theoretische Ansatz in Gleichung (4.1) zum einen nicht die vorhandene Inhomogenität des $\bar{u}$-Profils berücksichtigt und zum anderen die Zähigkeitseffekte vernachlässigt. Es ist anzunehmen, dass die Inhomogenität bei der Ausbreitung der KH-Wellen eine Rolle spielt, während die Zähigkeit eher bei der Dynamik der Ablösung von der Hinterkante wichtig ist.

$\underline{\text { Profile der Kelvin-Helmholtz-Wellen an verschiedenen Stellen hinter der Kante }}$

Wie man in Abbildung 3.4 erkennt, ist die Änderung des Gleichströmungsprofils in $x$-Richtung nahe der Hinterkante besonders groß und führt dazu, dass die komplexe Wellenzahl der KH-Wellen eine Abhängigkeit von der $x$-Koordinate erhält. Diese räumliche Abhängigkeit der Wellenzahl lässt sich allerdings näherungsweise durch die in Abschnitt 4.1 dargestellten 2D-Theorie bestimmen, in der $\partial \bar{u} / \partial x=0$ angenommen wird. Man erhält somit die lokalen Eigenfunktionen $\psi_{0}(x, y)$ bzw. die lokalen Wellenzahlen $\alpha(x)$ in Abhängigkeit von der $x$-Koordinate. Der Ansatz für $\tilde{\psi}_{K H}$ in Gleichung (4.7) lässt sich erweitern:

$$
\begin{aligned}
\tilde{\psi}_{K H}(x, y)=C_{A}(x) \psi_{0}(x, y) \exp & \left(i \int_{0}^{x} \alpha(x) d x\right) \\
& +C_{D}(x) \psi_{0}^{*}(x, y) \exp \left(i \int_{0}^{x} \alpha^{*}(x) d x\right)
\end{aligned}
$$

Die zwei Vorfaktoren $C_{A}$ und $C_{D}$, die durch Anpassung an Messwerte bestimmt werden, 


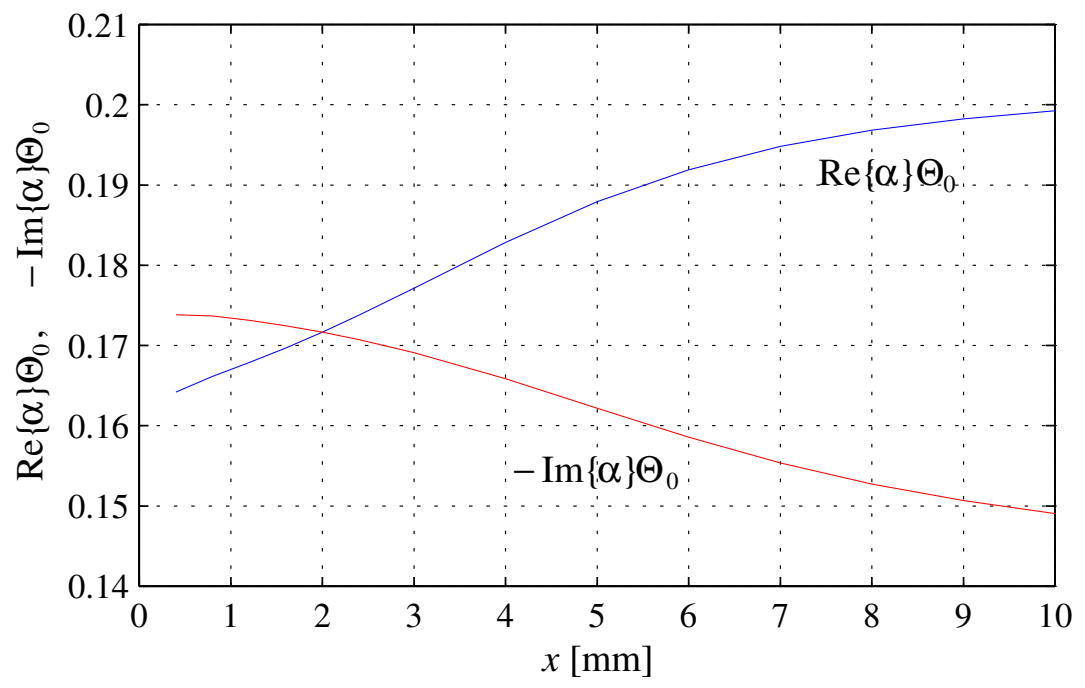

Abb. 4.17: Die durch die Rayleigh-Gleichung bestimmte lokale Wellenzahl (mit der Impulsverlustdicke $\Theta_{0}$ normiert) in Anhängigkeit von der $x$-Koordinate bei einer Anregungsfrequenz von $1.6 \mathrm{~Hz}$

erhalten daher auch eine Abhängigkeit von der $x$-Koordinate. Dieser Ansatz zur Bestimmung des Feldes $\tilde{\psi}_{K H}$ bedeutet, dass die Inhomogenität des $\bar{u}$-Profils hauptsächlich durch die $x$-Abhängigkeit des lokalen Eigenfunktionen berücksichtigt wird und die Korrektur für die aneinandergesetzten Eigenfunktionen durch die Anpassung an Messwerte, d. h. durch $C_{A}$ und $C_{D}$, durchgeführt wird.

Da man für die Anpassung an Messwerte theoretische Wechselgeschwindigkeiten der KH-Wellen $\tilde{u}_{K H}$ bzw. $\tilde{v}_{K H}$ benötigt, wird $\tilde{\psi}_{K H}$ nach $x$ und $y$ abgeleitet (hier wird dies aus Übersichtlichkeitsgründen nur für die angefachte Lösung $\tilde{u}_{K H_{A}}$ bzw. $\tilde{v}_{K H_{A}}$ dargestellt):

$$
\begin{aligned}
\tilde{u}_{K H_{A}} & =C_{A} \frac{\partial \psi_{0}}{\partial y} \exp \left(i \int_{0}^{x} \alpha(x) d x\right) \\
\tilde{v}_{K H_{A}} & =-\left(\frac{d C_{A}}{d x} \psi_{0}+C_{A} \frac{\partial \psi_{0}}{\partial x}+i \alpha \psi_{0}\right) \exp \left(i \int_{0}^{x} \alpha(x) d x\right)
\end{aligned}
$$

Man erkennt aus der Gleichung (4.22), dass $d C_{A} / d x$ zur Bestimmung $C_{A}$ berechnet werden muss. Die Anpassungen von $\tilde{u}_{K H_{A}}$ und $\tilde{v}_{K H_{A}}$ mit dem Parameter $C_{A}$ an gemessene Wechselgeschwindigkeiten wurde aufgrund des Terms $d C_{A} / d x$ in $\tilde{v}_{K H_{A}}$ iterativ durchgeführt: zuerst wird $C_{A}$ durch $\tilde{u}_{K H_{A}}$ für verschiedene $x$-Koordinaten gewonnen, danach wird $\tilde{v}_{K H_{A}}$ durch das so bestimmte $C_{A}$ berechnet. Bei der nächsten Iteration können daher $\tilde{u}_{K H_{A}}$ und $\tilde{v}_{K H_{A}}$ gleichzeitig berücksichtigt werden und somit wird $C_{A}(x)$ korrigiert. Diese Prozedur wiederholt sich solange, bis $C_{A}$ sich nicht mehr ändert.

Um den Term $\partial \psi_{0} / \partial x$ in $\tilde{v}_{K H_{A}}$ numerisch zu berechnen, benötigt man zudem eine glatte Funktion für die Gleichströmung $\bar{u}$ nicht nur bezüglich der $y$ - sondern auch bezüglich der $x$-Richtung. Die in $y$-Richtung differenzierbare Funktion (Gleichung (3.1)) wird 
mit noch fünf zusätzlichen Parametern entwickelt, so dass sie auch die Änderung des $\bar{u}$-Profils in $x$-Richtung beschreiben kann:

$$
\begin{aligned}
\bar{u}(x, y) & =u_{0}-u_{0}\left(1+m(x) e^{\frac{m(x)}{\Theta_{f}}\left(y-y_{0}(x)\right)}\right)^{-\frac{1}{m(x)}}+\left(u_{1} x+s\right) \\
& +\left[a\left(y-y_{1}\right)+b\left(y-y_{1}\right)^{2}+c\left(y-y_{1}\right)^{3}\right] / \cosh ^{2}\left(d\left(y-y_{2}\right)\right)
\end{aligned}
$$

wobei

$$
m(x)=n e^{p x}+q, \quad y_{0}(x)=f e^{g x}+h
$$

Abbildung 4.17 zeigt die Abhängigkeit der durch die Rayleigh-Gleichung bestimmten lokalen Wellenzahl von der $x$-Koordinate, die mit der Impulsverlustdicke $\Theta_{0}$ normiert ist. Die Anregungsfrequenz ist dabei $1.6 \mathrm{~Hz}$. Bei dieser Frequenz ist die Anfachungskonstante etwa maximal. Mit steigender $x$-Koordinate nimmt sie allmählich ab. Die Phasenkonstante nimmt dagegen mit laufendem Weg zu, die Phasengeschwindigkeit wird mit steigender $x$-Koordinate kleiner.

Nun wird der Versuch unternommen, das Feld $\tilde{\psi}_{K H}$ mit dem Ansatz (Gleichung (4.21)) zu bestimmen. Die zwei Parameter $C_{A}(x)$ und $C_{D}(x)$ werden durch Anpassung an Messwerte $\tilde{u}_{K H+N F-s}^{D r}$ und $\tilde{v}_{K H+N F-s}^{D r}$ so bestimmt, dass das Quadrat der Abweichung zwischen den Mess- und den Theoriewerten sowohl für die $\tilde{u}$-als auch für die $\tilde{v}$-Komponente nur im Potenzialbereich, also $y<-5 \mathrm{~mm}$ und $y>10 \mathrm{~mm}$, minimal wird, da zu erwarten ist, dass das Nahfeld $\tilde{\psi}_{N F}^{D r}$, das im Messfeld insbesondere innerhalb der Scherschicht vorhanden sein kann, keine Ähnlichkeit mit $\tilde{\psi}_{K H}^{D r}$ hat.

Die Abbildungen 4.18 und 4.19 zeigen den Vergleich zwischen Messwerten und Theoriekurven für Beträge und Phasen der $\tilde{u}$ - bzw. $\tilde{v}$-Komponente bei verschiedenen $x$ Koordinaten. Das Wechselfeld wurde dabei durch den Druckgradient angeregt und die $x$-Koordinaten sind bei der Darstellung von oben nach unten (a) $0.4 \mathrm{~mm}$, (b) $3 \mathrm{~mm}$, (c) $6 \mathrm{~mm}$ und (d) $10 \mathrm{~mm}$.

Es ist zu erkennen, dass einerseits die Abweichung zwischen den Messwerten und den Theoriekurven sowohl im Betrag als auch in der Phase in der Scherschicht mit abnehmender $x$-Koordinate größer wird. Man sieht auch eine systematische Abweichung bei der $\tilde{v}$-Komponente im Hauptströmungsbereich, also $3 \mathrm{~mm} \leq y \leq 10 \mathrm{~mm}$. Der Betrag der Messwerte liegt in diesem Bereich unter der theoretischen Kurve und die Abweichung ist bei $x=0.4 \mathrm{~mm}$ am größten. Andererseits stimmen die theoretischen Kurven im Potenzialbereich, besonders im Nebenströmungsbereich, im wesentlichen mit den Messwerten unabhängig von der $x$-Koordinate überein.

Man kann daher davon ausgehen, dass das Messfeld zum einen im Bereich nahe der Hinterkante, wie man vermutet, nicht vollständig durch das anregende Feld $\tilde{\psi}_{A n F}^{D r}$ und das Feld der Instabilitätswellen $\tilde{\psi}_{K H}^{D r}$ beschrieben wird, sondern darüberhinaus ein Nahfeld $\tilde{\psi}_{N F}^{D r}$ enthalten. Zum anderen wird das Messfeld außerhalb der Scherschicht schon dicht stromab der Hinterkante durch die Instabilitäts-Wellen dominiert. Man darf allerdings nicht aus der Differenz von $\tilde{u}_{K H+N F-s}^{D r}$ und $\tilde{u}_{K H}^{D r}$ das Nahfeld $\tilde{u}_{N F}^{D r}$ bestimmen, da die scheinbare Geschwindigkeit $\tilde{u}_{s}^{D r}$ auch aufgrund des Ansatzes (Gleichung (4.5)) zum Nahfeld gehört. $\tilde{v}_{N F}^{D r}$ kann dagegen aus der Differenz von $\tilde{v}_{K H+N F-s}^{D r}$ und $\tilde{v}_{K H}^{D r}$ bestimmt werden, da hier $\tilde{v}_{s}^{D r}=0$ angesetzt wird (siehe Gleichung (4.14)). 

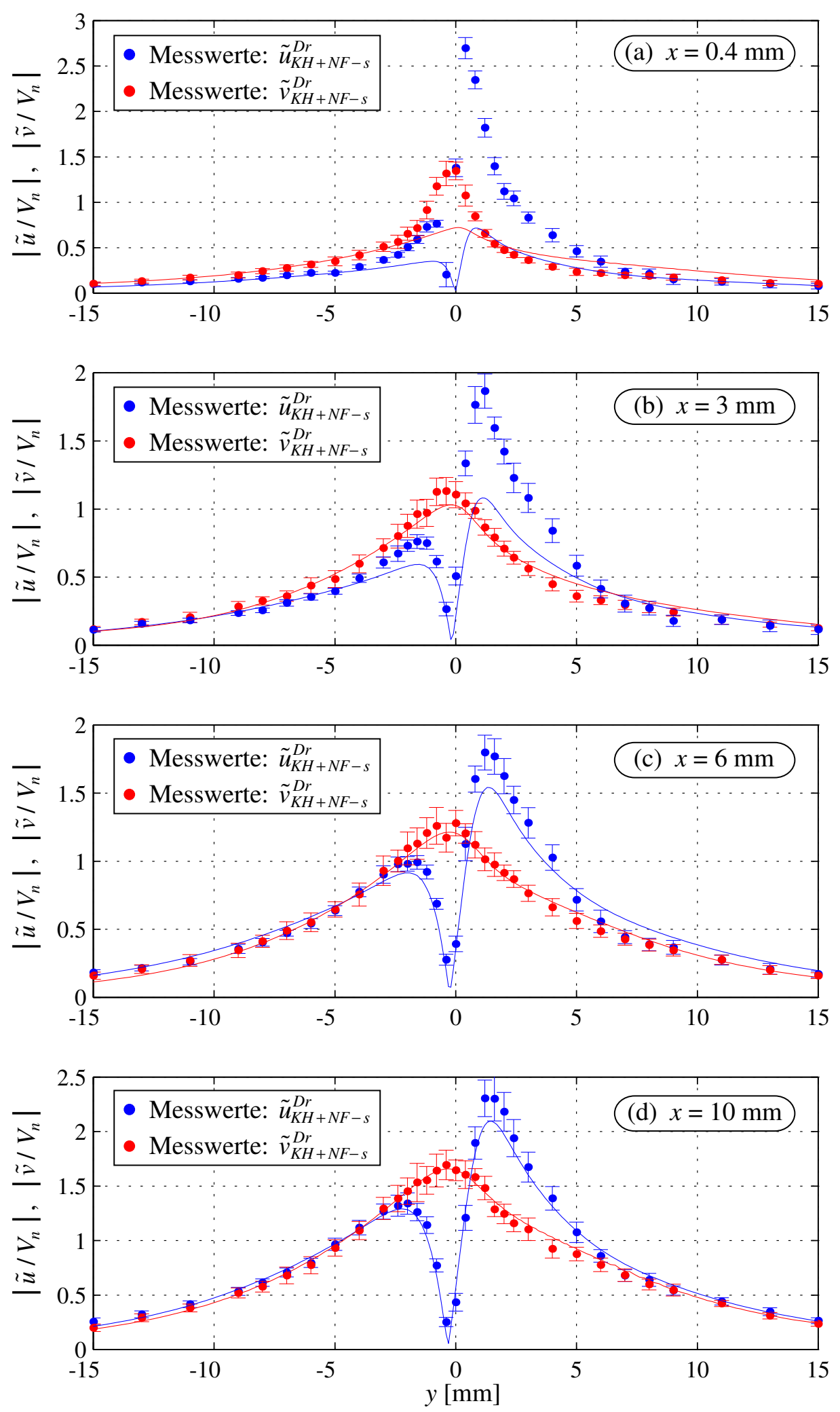

Abb. 4.18: Vergleich der Messwerte $\left|\tilde{u}_{K H+N F-s}^{D r}\right|,\left|\tilde{v}_{K H+N F-s}^{D r}\right|$ mit den durch Ansatz (Gleichung (4.21)) bestimmten Theoriekurven $\left|\tilde{u}_{K H}^{D r}\right|,\left|\tilde{v}_{K H}^{D r}\right|$ in der Scherschicht bei verschiedenen $x$-Koordinaten für $1.6 \mathrm{~Hz}$ : (a) $x=0.4 \mathrm{~mm}$, (b) $x=3 \mathrm{~mm}$, (c) $x=6 \mathrm{~mm}$ und (d) $x=10 \mathrm{~mm}$ 

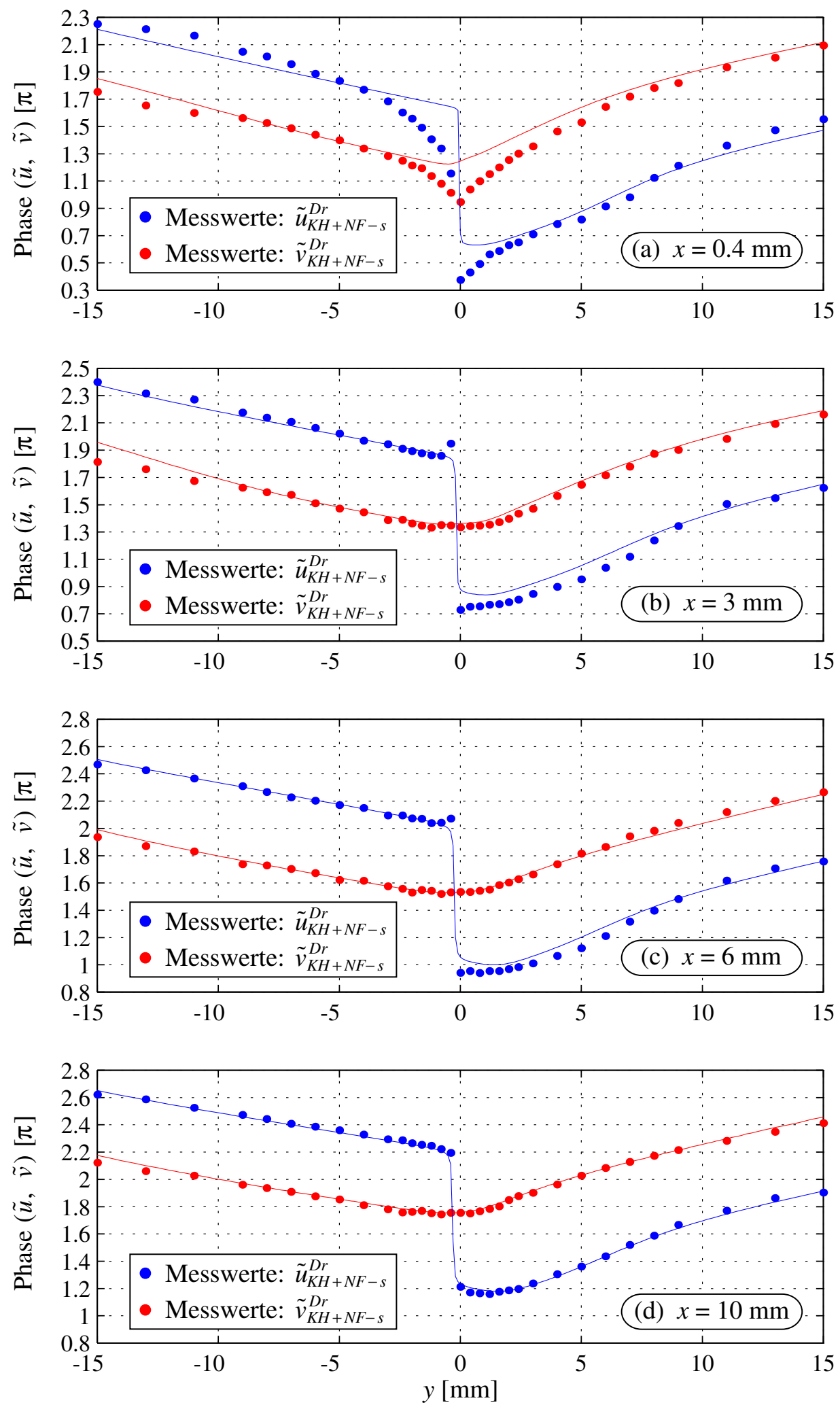

Abb. 4.19: Vergleich der gemessenen Phasen von $\tilde{u}_{K H+N F-s}^{D r}$ und $\tilde{v}_{K H+N F-s}^{D r}$ mit den durch Ansatz (Gleichung (4.21)) bestimmten Theoriekurven $\widetilde{u}_{K H}^{D r}, \tilde{v}_{K H}^{D r}$ in der Scherschicht bei verschiedenen $x$-Koordinaten für $1.6 \mathrm{~Hz}$ : (a) $x=0.4 \mathrm{~mm}$, (b) $x=3 \mathrm{~mm}$, (c) $x=6 \mathrm{~mm}$ und (d) $x=10 \mathrm{~mm}$ 

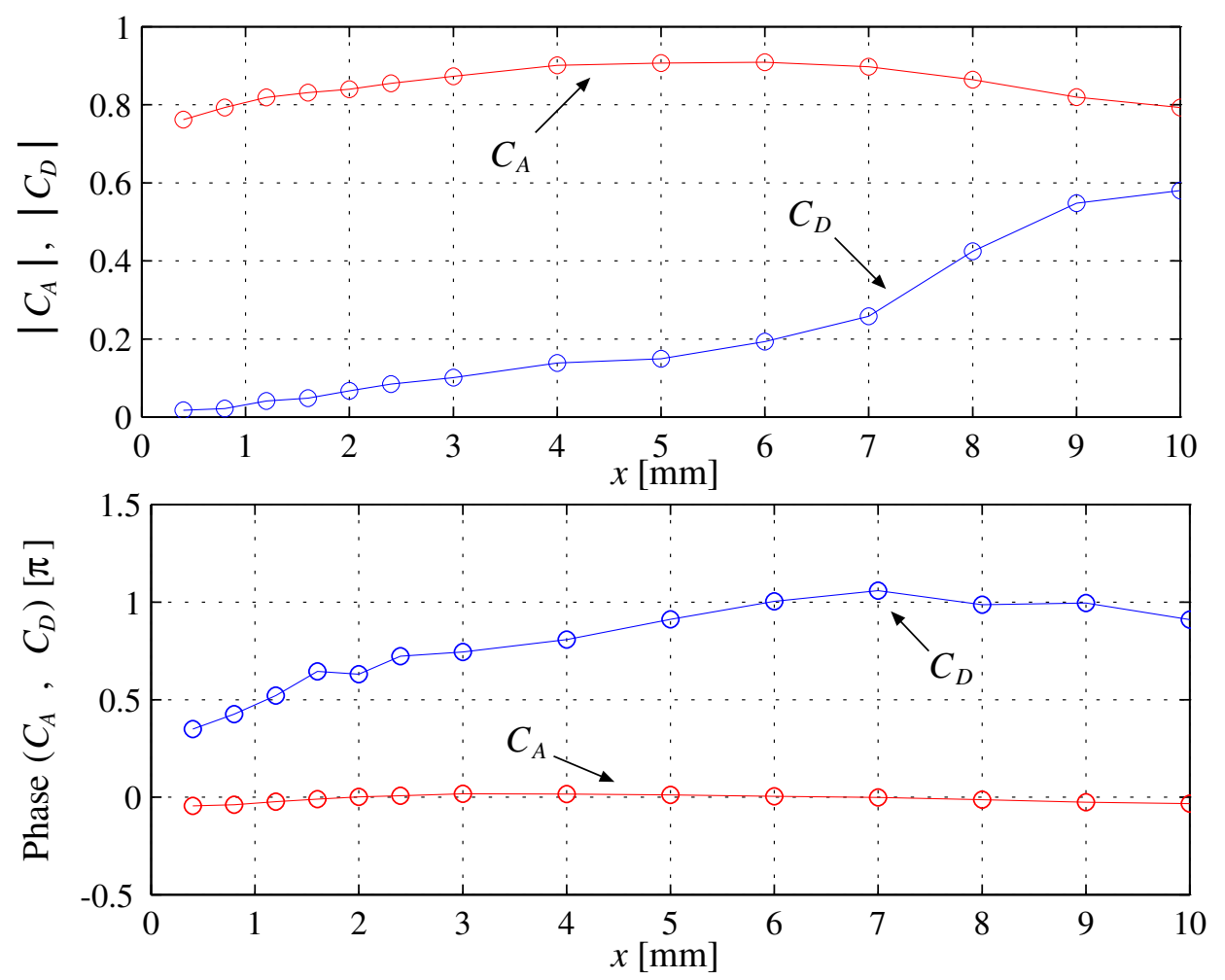

Abb. 4.20: Die Abhängigkeit der durch Anpassung bestimmten Vorfaktoren $C_{A}$ und $C_{D}$ von der $x$-Koordinate (Oben: Betrag, unten: Phase).

Da das Verhältnis zwischen der angefachten und der gedämpften Lösung der KHWellen ein wichtiger Parameter ist, um $\tilde{\psi}_{K H}$ zu bestimmen, werden hier die durch die Anpassungen bestimmten Vorfaktoren $C_{A}$ und $C_{D}$ diskutiert, bevor das Nahfeld bestimmt wird. Abbildung 4.20 zeigt den Betrag und die Phase von $C_{A}$ und $C_{D}$ in Abhängigkeit von der $x$-Koordinate, wobei die Anregungfrequenz $1.6 \mathrm{~Hz}$ ist. Man sieht, dass $C_{A}$ in Strömungsrichtung sowohl im Betrag als auch in der Phase etwa konstant bleibt. Das bedeutet, dass die Abhängigkeit der angefachten Lösung der KH-Welle von der $x$-Koordinate hauptsächlich durch die lokale Wellenzahl beschrieben wird. $C_{D}$ ändert sich dagegen mit steigender $x$-Koordinate systematisch. Nahe der Hinterkante ist $C_{D}$ ziemlich klein und somit ist anzunehmen, dass dort $\tilde{\psi}_{K H}^{D r}$ nur durch die angefachte Mode beschrieben wird. Weshalb der Betrag von $C_{D}$ mit steigender $x$-Koordinate zunimmt, kann vermutlich dadurch erklärt werden, dass die angefachte Mode infolge der Inhomogenität in die gedämpfte Mode gestreut wird, besonders weiter stromab der Hinterkante, wo sich die Instabilitätswelle voll entwickelt hat und die Inhomogenität noch wesentlich ist. Diese Wechselwirkung zwischen den beiden Moden führt dazu, dass die gedämpfte KH-Welle aufgrund der angefachten KH-Welle außerhalb des Kantenbereiches nicht exponentiell mit steigender $x$-Koordinate abnimmt. 
$\underline{\text { Berücksichitigung der Inhomogenität mittels der WKB-Methode }}$

Um die durch Anpassung bestimmten Vorfaktoren $C_{A}$ und $C_{D}$ auf eine sichere Basis zu stellen, wird hier die Berücksichtigung der Inhomogenität des $\bar{u}$-Profils theoretisch untersucht. Als ein Maß für die nicht parallele Gleichströmung wird ein Parameter $\varepsilon$ eingeführt, der definiert ist als:

$$
\varepsilon \sim k \cdot|\alpha|^{-1} \quad \text { wobei } \quad k(x)=\frac{1}{U_{1}}\left|\frac{\partial \bar{u}}{\partial x}\right|_{\max }
$$

$|\partial \bar{u} / \partial x|_{\max }$ ist das Maximum des $|\partial \bar{u} / \partial x|(y)$-Profils und somit $k(x)$ ein Maß für die relative Änderung der Gleichströmung in $x$-Richtung. Ist nun anzunehmen, dass sich das Strömungsprofil im Vergleich zu $\tilde{u}$ nur langsam ändert $(k \ll|\alpha|$, also $\varepsilon \ll 1)$, kann das Feld der KH-Wellen für die angefachte Lösung $\tilde{\psi}_{K H_{A}}(x, y)$ multiplikativ in einen langsam stromab veränderlichen Teil $\psi_{0}(x, y)$ und in einen schnell variierenden Teil $\exp \left(i \int_{0}^{x} \alpha(x) d x\right)$ zerlegt werden:

$$
\tilde{\psi}_{K H_{A}}(x, y)=a_{0} A(x) \psi_{0}(x, y) \exp \left(i \int_{0}^{x} \alpha(x) d x\right)
$$

Diese Gleichung bedeutet, dass die komplexe Amplitude $A(x)$ versucht die lokalen Eigenfunktionen $\psi_{0}(x, y)$ glatt aneinanderzusetzen. Hierbei ist $a_{0}$ ein freier Parameter. Setzt man Gleichung (4.25) in die linearisierte zweidimensionale Euler-Gleichung ein, und betrachtet die Terme der Ordnung $\varepsilon$, so erhält man mittels der WKB-Methode die folgende Differenzialgleichung für $A(x)$ (Gaster, Kit \& Wygnanski [11], GodreCHE \& MANNEVILLE [12]):

$$
A(x) N(x)+\frac{d A(x)}{d x} M(x)=0
$$

wobei

$$
\begin{aligned}
& N(x)=\int_{-\infty}^{+\infty}\left\{\beta\left[\psi_{0} \frac{d \alpha}{d x}+2 \alpha \frac{\partial \psi_{0}}{\partial x}\right]+\bar{u}\left[\frac{\partial \psi_{0}^{\prime \prime}}{\partial x}-3 \alpha^{2} \frac{\partial \psi_{0}}{\partial x}-3 \alpha \psi_{0} \frac{d \alpha}{d x}\right]\right. \\
& \left.+\psi_{0}^{\prime} \frac{\partial \bar{u}^{\prime}}{\partial x}-\bar{u}^{\prime \prime} \frac{\partial \psi_{0}}{\partial x}+\bar{v}\left[\psi_{0}^{\prime \prime \prime}-\alpha^{2} \psi_{0}^{\prime}\right]\right\} \psi_{0}^{*} d y
\end{aligned}
$$

und

$$
M(x)=\int_{-\infty}^{+\infty}\left\{2 \alpha \beta \psi_{0}+\bar{u}\left[\psi_{0}^{\prime \prime}-3 \alpha^{2} \psi_{0}\right]-\bar{u}^{\prime \prime} \psi_{0}\right\} \psi_{0}^{*} d y
$$

Für die gedämpfte Lösung $\tilde{\psi}_{K H_{D}}(x, y)$ kann der Ansatz (Gleichung (4.25)) auch verwendet werden, in dem jedoch $\alpha=\alpha^{*}$ und $\psi_{0}=\psi_{0}^{*}$ eingesetzt werden müssen. Man erhält somit die Beziehung $D(x)=A^{*}(x)$, wobei $D(x)$ die durch die WKB-Methode bestimmte komplexe Amplitude für die gedämpfte Lösung ist. Es ist aus dieser Beziehung allerdings nicht zu erwarten, dass mit dem Ansatz die Wechselwirkung zwischen den 


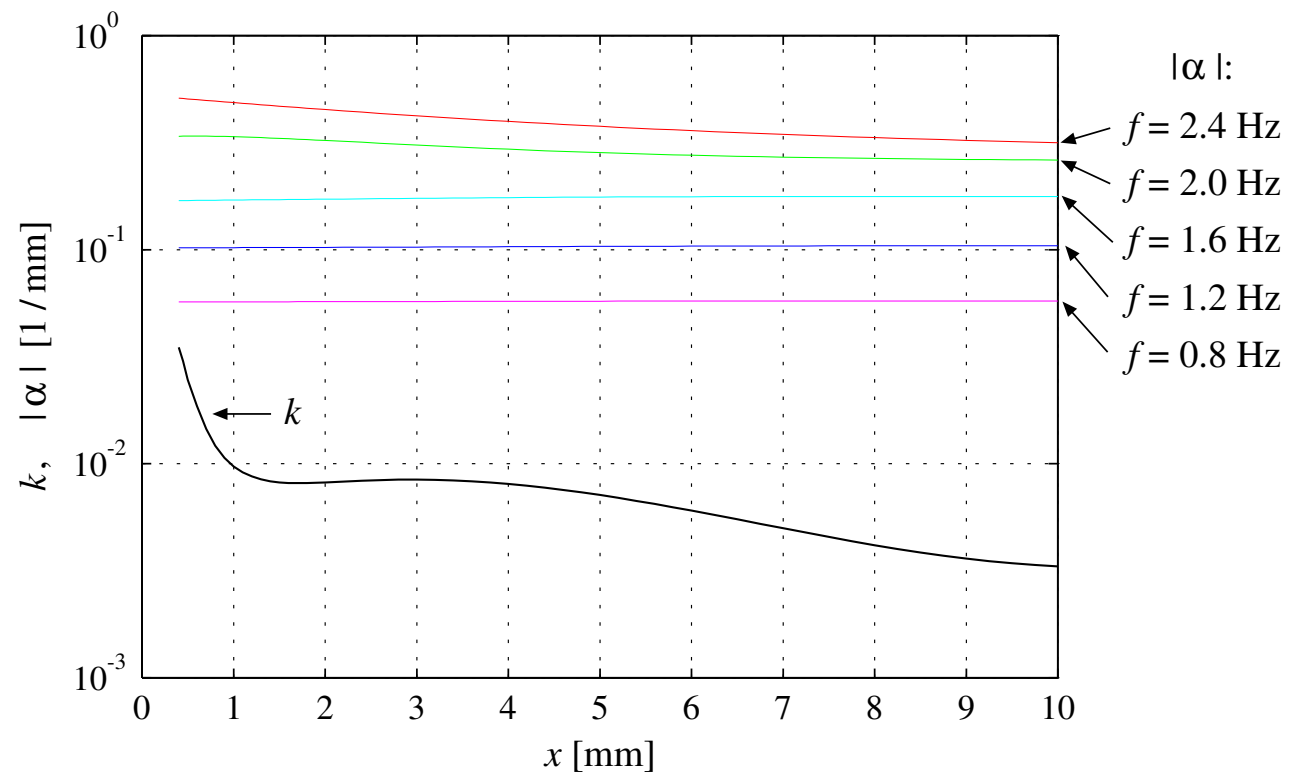

Abb. 4.21: Vergleich der relativen Änderung des $\bar{u}$-Profils in $x$-Richtung $k$ mit der Wellenzahl $|\alpha|$ bei verschiedenen Frequenzen

beiden Moden berücksichtigt werden kann. Da anzunehmen ist, dass die gedämpfte Mode sehr wenig in die angefachte Mode gestreut wird, wird hier der Vergleich der Vorfaktoren $C_{A}(x)$ und $A(x)$, also nur für die angefachte Lösung, gezeigt.

Zuerst werden in Abbildung 4.21 die relative $x$-Gradient des $\bar{u}$-Profils $k(x)$ und die Wellenzahl $|\alpha|$ bei fünf verschiedenen Anregungsfrequenzen gezeigt. Aus der Auftragung erkennt man, in welchem Bereich der $x$-Koordinate und bei welcher Frequenz die Bedingung des Ansatzes für die WKB-Methode, also $\varepsilon \ll 1$, erfüllt ist. Da bei den tiefen Frequenzen $(f=0.8 \mathrm{~Hz}$ und $f=1.2 \mathrm{~Hz})$ die Wellenzahlen klein sind, und außerdem die Inhomogenität des $\bar{u}$-Profils für kleine $x$ sehr stark ist, ist der Ansatz mit der WKB-Methode bei den tiefen Frequenzen für die Beschreibung $\tilde{\psi}_{K H}$ im Bereich direkt an der Hinterkante nicht geeignet, bzw. kann $A(x)$ keine vernünftigen Werte liefern. Dagegen ist bei den höheren Frequenzen $(f=2.0 \mathrm{~Hz}$ und $f=2.4 \mathrm{~Hz})$ anzunehmen, dass $\varepsilon \ll 1$ für $x>0$ aufgrund der Zunahme der Wellenzahl erfüllt ist.

Der Vergleich zwischen $C_{A}(x)$ und $A(x)$ ist in Abbildung 4.22 (oben: Betrag, unten: Phase) zu sehen. $A(x)$ wurde dabei an $C_{A}(x)$ global angepasst, wobei die Abweichungen mit $\varepsilon^{-1}$ gewichtet wurden. Man sieht, dass der mittels der WKB-Methode bestimmte Vorfaktor $A$ bei den tiefen Frequenzen $(f=0.8 \mathrm{~Hz}$ und $f=1.2 \mathrm{~Hz})$ auf der $x$-Koordinate fast konstant ist, während das durch Anpassung bestimmte $C_{A}$ im Bereich etwa $0 \leq x \leq 5 \mathrm{~mm}$ eine deutliche Abhängigkeit von der $x$-Koordinate zeigt. Bei $1.6 \mathrm{~Hz}$ stimmt $C_{A}$ mit $A$ für große $x$ überein, wo $\varepsilon \ll 1$ deutlich erfüllt ist. Bei den grünen Kurven $(2.0 \mathrm{~Hz})$ erkennt man, dass $C_{A}$ durch die WKB-Lösung wesentlich besser wiedergegeben wird. Auch bei $2.4 \mathrm{~Hz}$ findet man Übereinstimmung, wobei $C_{A}$ aufgrund des kleinen Betrages ein wenig streut, besonders in der Nähe der Hinterkante. Es fällt weiterhin auf, dass der Betrag von $C_{A}$ mit abnehmender Anregungsfrequenz 

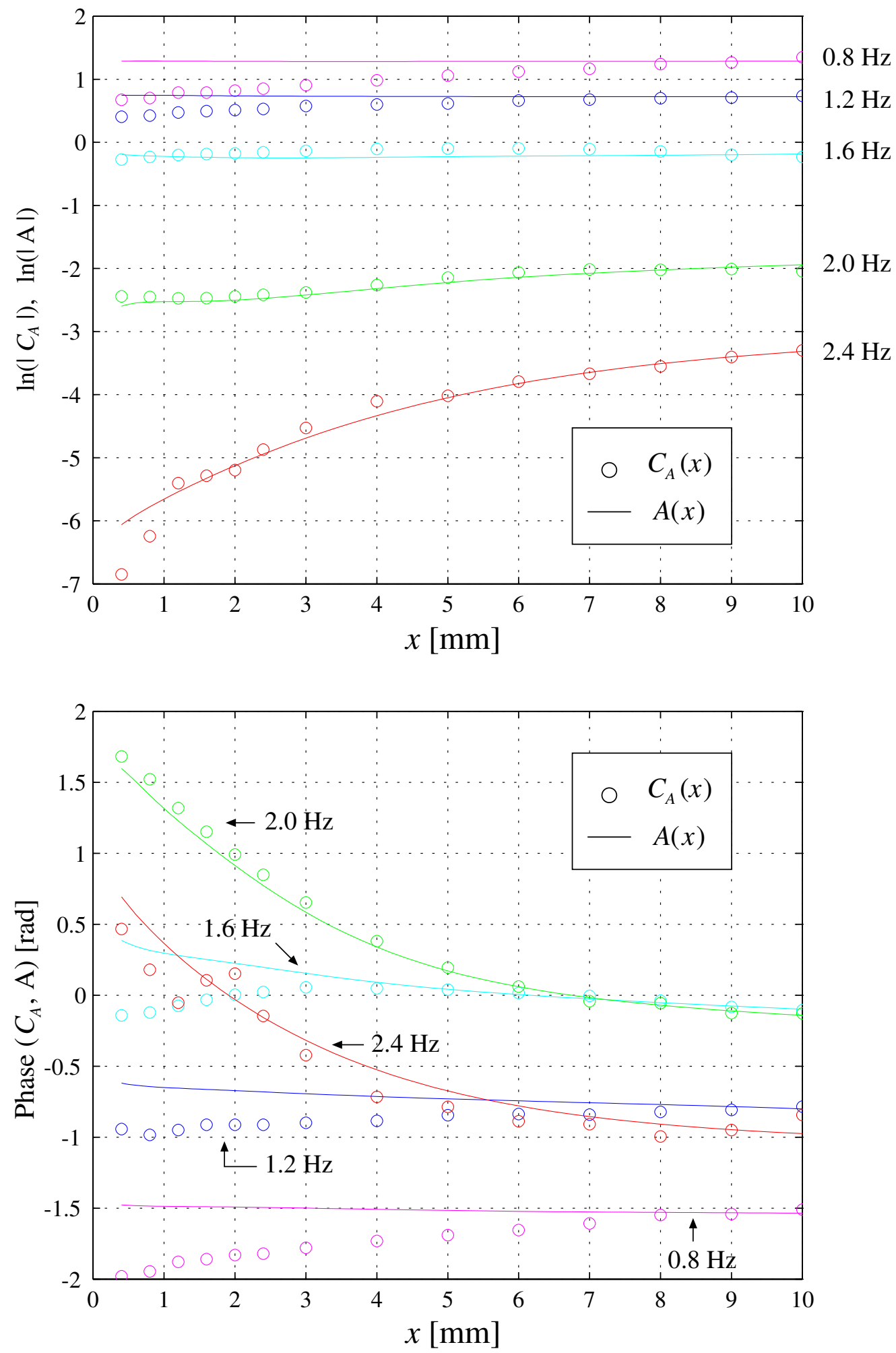

Abb. 4.22: Vergleich der Vorfaktoren $C_{A}(x)$ (Anpassung an Messwerte) mit $A(x)$ (WKB-Methode) für die angefachte Lösung der KH-Welle bei fünf verschiedenen Frequenzen 

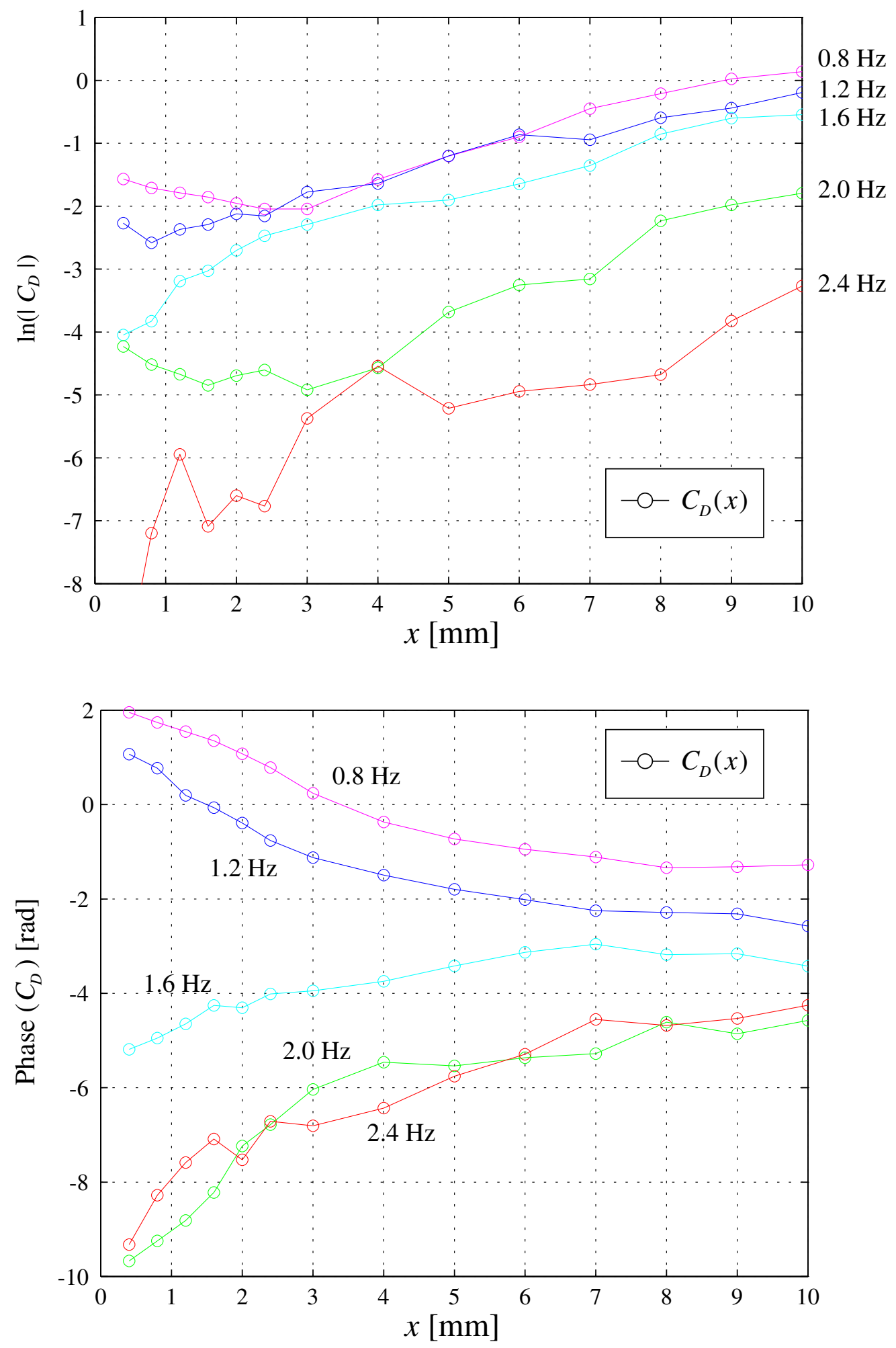

Abb. 4.23: Der durch Anpassung an Messwerte bestimmte Vorfaktor $C_{D}(x)$ für die gedämpfte Lösung der KH-Welle 
anwächst. Da die gemessenen Wechselfelder bei allen Frequenzen mit gleicher Geschwindigkeit der Membranen der Druckgradienten-Anregung erzeugt wurden, bedeutet das Ergebnis, dass die lokale Amplitude $C_{A}$ bei $0.8 \mathrm{~Hz}$ am größten ist. Dies könnte mit der Ablösungsbedingung im Zusammenhang stehen. Wie im Abschnitt 4.1.2 erwähnt wurde, wird im quasistationären Fall die $\tilde{v}$-Komponente stark unterdrückt, so dass die Strömung an der Hinterkante tangential ablöst. Es lässt sich vermuten, dass sich die durch die Ablösebedingung verursachte Umverteilung des Strömungsfeldes in die lokale Amplitude der KH-Welle wandelt.

Abbildung 4.23 (oben: Betrag, unten: Phase) zeigt die Vorfaktoren $C_{D}$ für die gedämpfte Mode bei fünf verschiedenen Frequenzen, die zu den gerade dargestellten $C_{A}$ gehören. Bei dem Betrag ist zu deutlich erkennen, dass $C_{D}$ für alle Frequenzen mit steigender $x$-Koordinate anwächst. Wie im Zusammenhang mit Abbildung 4.20 vermutet wurde, stellt man somit fest, dass die gedämpfte Mode von der angefachten Mode mit zunehmendem Laufweg beeinflusst wird. Der Abhängigkeit des Betrags von der Frequenz ergibt sich hier auch wie bei $C_{A}$, und die lokale Amplitude $C_{D}$ ist somit bei $0.8 \mathrm{~Hz}$ am größten.

Die Phasen zeigen für kleine $x$ eine nennenswerte Abhängigkeit von der $x$-Koordinate, während sie sich mit steigendem $x$ etwa konstanten Werten annähern. Obwohl die Phase bei $x=10 \mathrm{~mm}$ eine systematische Abhängigkeit von der Frequenz enthält, ist eine einfache Beziehung zwischen $C_{A}$ und $C_{D}$ an dieser Stelle nicht erkennbar.

\subsection{4 Überblick über das Nahfeld}

Im letzten Abschnitt wurde versucht das Feld der KH-Wellen $\tilde{\psi}_{K H}$ zu beschreiben. Aufgrund der Inhomogenität der Gleichströmung ist die Beschreibung des Feldes besonders im Bereich nahe der Hinterkante schwierig. Zur Berücksichtigung der Inhomogenität wurde der Ansatz (Gleichung (4.21)) verwendet, in dem die Abhängigkeit der KH-Wellen von der $x$-Koordinate nicht nur durch die lokale Wellenzahl $\left(\alpha, \alpha^{*}\right)$ sondern auch die lokalen Eigenfunktionen $\left(\psi_{0}, \psi_{0}^{*}\right)$ sowie die lokale Amplitude $\left(C_{A}, C_{D}\right)$ nährungsweise beschrieben wird. $C_{A}$ und $C_{D}$ werden dabei durch Anpassung an die Messwerte $\tilde{u}_{K H+N F-s}$ bzw. $\tilde{v}_{K H+N F-s}$ bestimmt. Unter der Annahme, dass $\tilde{\psi}_{K H}$ nur aus der angefachten Lösung besteht, kann $C_{A}$ auch theoretisch mittels der WKB-Methode bestimmt werden. Der Vergleich der beiden lokalen Amplituden hat gezeigt, dass das durch die Anpassung bestimmte $C_{A}(x)$ durch das theoretische $A(x)$ im wesentlichen richtig wiedergegeben wird. Da die gedämpfte Lösung, die vermutlich von der angefachten Lösung beeinflusst wird, für die Beschreibung von $\tilde{\psi}_{K H}$ notwendig ist, wird hier das gemäß Ansatz (Gleichung (4.21)) angepasste Feld verwendet.

Das Nahfeld $\tilde{\psi}_{N F}$ kann nun genäß Gleichung (4.5) bestimmt werden. Da $\tilde{\psi}_{K H}$ ausschließlich für $x>0 \mathrm{~mm}$ bestimmt wird, erhält man $\tilde{u}_{N F}^{D r}$ und $\tilde{v}_{N F}^{D r}$ nur in diesem Bereich. Abbildung 4.24 zeigt somit den normierten Betrag der beiden Geschwindigkeitskomponenten, die durch die Druckgradienten-Anregung bei 1.6 Hz erzeugt wurden, im Bereich stromab der Hinterkante.

Bei der $\tilde{u}$-Komponente ist deutlich zu erkennen, dass sich eine signifikante Struktur im Hauptströmungsbereich befindet. Dieser Nahfeldanteil entsteht vermutlich durch 

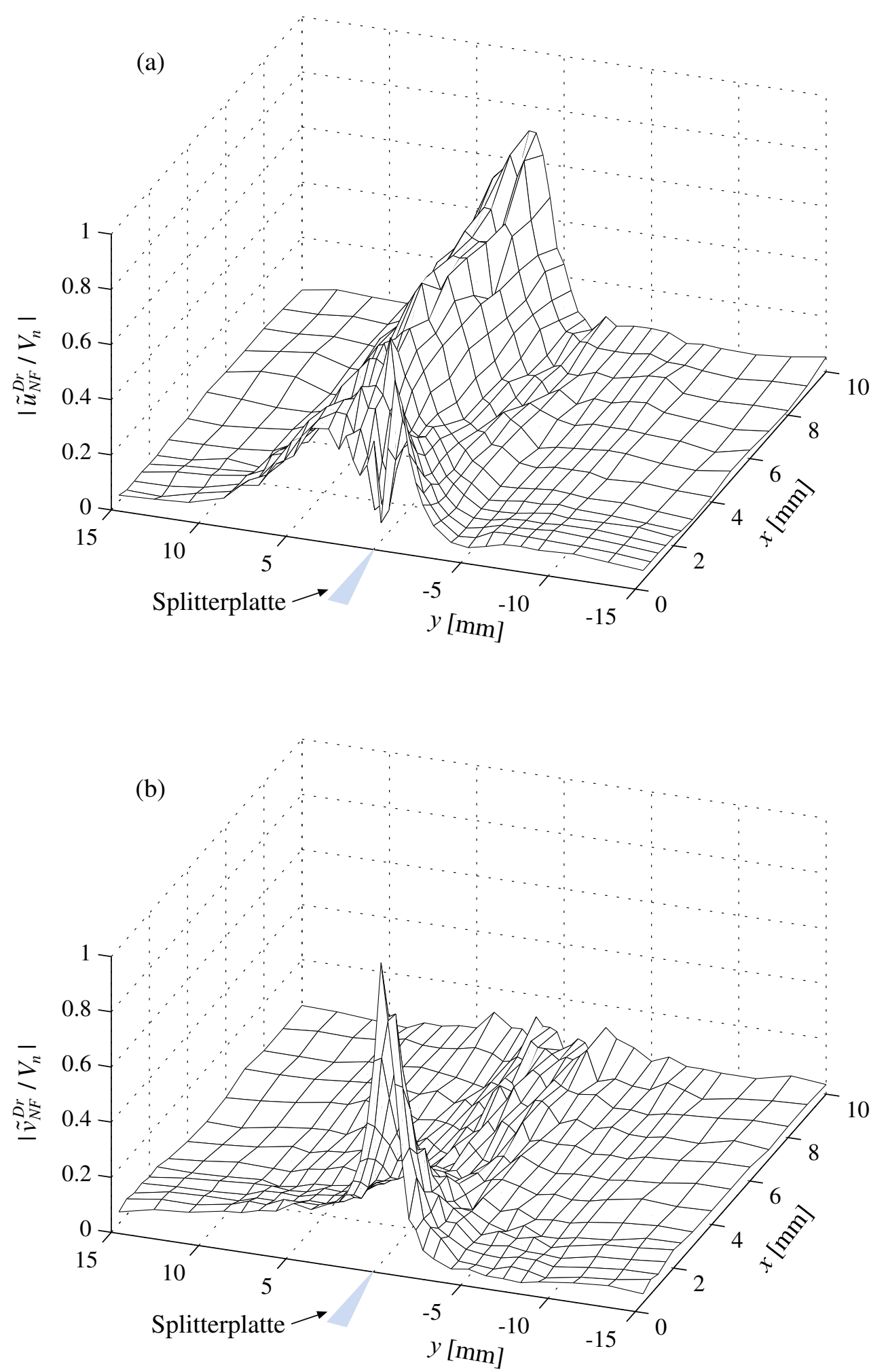

Abb. 4.24: Übersicht über den Betrag des durch die Druckgradienten-Anregung erzeugten Nahfeldes bei $1.6 \mathrm{~Hz}$ : (a) die $\tilde{u}$ - und (b) die $\tilde{v}$-Komponente 
die Verschiebung des $\bar{u}$-Profils und wird hauptsächlich durch die scheinbare Geschwindigkeit $\tilde{u}_{s}$ beschrieben. Die $\tilde{v}$-Komponente hat einen großen "Peak" im Bereich dicht hinter der Ablösekante, während keine auffällige Struktur außerhalb dieses Bereiches bei der Darstellung erkennbar ist.

Die Frage ist nun, woraus genau das Nahfeld im einzelnen besteht. Mit anderen Worten, ob es eine bestimmte Struktur in dem Feld gibt, die z. B. von der Anregungsfrequenz abhängig ist. Die Beantwortung dieser Frage könnte dazu führen, dass man eine Größe im Nahfeld finden kann, auf die man die Amplitude der erzeugten KH-Welle beziehen kann. Die Untersuchung des Nahfeldes wird zu dieser Zwecke in dem nächsten Kapitel durchgeführt. 



\title{
Kapitel 5
}

\section{Untersuchung der Nahfelder mittels Kompensationsexperiment}

\begin{abstract}
Das Nahfeld, das durch die in Kapitel 4 vorgestellte Zerlegung des gemessenen Feldes bestimmt wurde, soll in diesem Kapitel genauer untersucht werden. Dazu wurde ein Kompensationsexperiment durchgeführt. Die grundlegende Idee des Kompensationsexperiments ist es, zwei durch unterschiedliche Anregungen ((in der vorliegenden Arbeit) die Schwingkanten- und die Druckgradienten-Anregung) bewirkte Wechselfelder so zu überlagern, dass die Amplitude der resultierenden Wechselgeschwindigkeit zumindest an einem Fernfeldpunkt verschwindet. Nimmt man an, dass das Fernfeld idealerweise nur durch einen Freiheitsgrad, also das durch eine komplexe Amplitude festgelegte Feld der angefachten Instabilitätswelle, beschrieben wird, ist zu erwarten, dass ein nicht verschwindendes, nicht anregendes Nahfeld im kompensierten Wechselfeld vorhanden ist, da die Schwingkanten- und die Druckgradienten-Anregung bei ruhendem Medium voneinander abweichende Wechselströmungen bewirken. Die Frage ist nun, ob eine Struktur in dem aus dem Kompensationsexperiment gewonnenen Nahfeld zu erkennen ist, die mit dem im vorigen Kapitel bestimmten Nahfeld übereinstimmt. Eine weitere Frage ist, ob aus dem Nahfeld Rückschlüsse auf die anregenden Größen der KH-Instabilität gezogen werden können.
\end{abstract}

\subsection{Das Kompensationsexperiment}

Bei der Durchführung des Experiments wurde die komplexe Kompensationsamplitude der Druckgradienten-Anregung $M$ mit Hilfe eines Gradientenverfahrens so ermittelt, dass die Amplitude der durch die Schwingkanten-Anregung erzeugten $\tilde{v}$-Komponente der Wechselgeschwindigkeit an einer Stelle $(x, y=0)$ im Fernfeld minimal wird. Die $\tilde{v}$-Komponente eignet sich besser als die $\tilde{u}$-Komponente für die Ermittelung von $M$, da die $\tilde{u}$-Komponente eventuell noch die scheinbare Geschwindigkeit $\tilde{u}_{s}$ enthalten könnte, die die genaue Bestimmung von $M$ behindert. $y=0$ ist deshalb ausgewählt, da sich etwa dort das Maximum der $\tilde{v}$-Komponente der angefachten KH-Welle befindet.

Das gesamte Kompensationsfeld $\tilde{\psi}_{A l l}^{K o m}$ kann nun als eine Überlagerung zweier Felder angesehen werden, die im folgenden beschrieben wird: 


$$
\tilde{\psi}_{A l l}^{K o m}(x, y)=\tilde{\psi}_{A l l}^{K l}(x, y)+M \cdot \tilde{\psi}_{A l l}^{D r}(x, y),
$$

wobei $\tilde{\psi}_{A l l}^{K l}$ das durch die Schwingkanten-Anregung und $\tilde{\psi}_{A l l}^{D r}$ das durch die Druckgradienten-Anregung erzeugte gesamte Wechselfeld ist.

\subsubsection{Die Qualität der Kompensation}

Das Kompensationsexperiment wurde bei einer Frequenz von $1.6 \mathrm{~Hz}$ durchgeführt, wobei $M$ durch Kompensation bei $x=30 \mathrm{~mm}$ bestimmt wurde. Abbildung 5.1 stellt die Wechselgeschwindigkeiten $\left(\tilde{u}_{A l l}^{\text {Kom }}\right.$ bzw. $\left.\tilde{v}_{A l l}^{\text {Kom }}\right)$ im Kompensationsfeld bei $x=30 \mathrm{~mm}$ in Abhängigkeit von der $y$-Koordinate dar. Zum Vergleich wurden auch die primären Felder $\tilde{u}_{A l l}^{K l}$ und $M \cdot \tilde{u}_{A l l}^{D r}$ bzw. $\tilde{v}_{A l l}^{K l}$ und $M \cdot \tilde{v}_{A l l}^{D r}$ in die entsprechenden Teilabbildungen eingezeichnet. Die Abbildungen 5.1 (a) und (c) zeigen die mit $V_{k}$ normierten Beträge, (b) und (d) die dazugehörigen Phasen. Die Phasen sind dabei auf die Geschwindigkeit der jeweiligen Anregungsvorrichtung bezogen, und die Phase für das Kompensationsfeld auf die der Schwingkante. Die durch $M$ eingestellten Profile $\tilde{u}_{A l l}^{D r}$ und $\tilde{v}_{A l l}^{D r}$ stimmen mit den Profilen $\tilde{u}_{A l l}^{K l}$ und $\tilde{v}_{A l l}^{K l}$ bis auf die entgegengesetzten Phasen sehr gut überein. Die zur Anregung kohärenten Wechselgeschwindigkeiten im Kompensationsfeld sind somit auf dieser Linie $(x=30 \mathrm{~mm})$ sehr klein (sowohl für die $\tilde{u}$ - als auch die $\tilde{v}$-Komponente liegt der Betrag bei max. ca. $6 \%$ der Amplitude von $\tilde{u}_{A l l}^{K l}$ bzw. $\tilde{v}_{A l l}^{K l}$ ). Aus dieser Darstellung kann man davon ausgehen, dass zum einen die Qualität der Kompensation unabhängig von der Richtung der Wechselgeschwindigkeit ist und dass zum anderen die beiden Anregungsvorrichtungen ein und dieselbe angefachte Mode der KH-Wellen anregen.

Man interessiert sich nun für das gesamte Kompensationsfeld im Bereich stromab der Hinterkante. Abbildung 5.2 zeigt die Beträge der $\tilde{u}$ - bzw. der $\tilde{v}$-Komponente bei $1.6 \mathrm{~Hz}$ in einer dreidimensionalen Darstellung für das gesamte Kompensationsfeld. Das Profil bei $x=30 \mathrm{~mm}$ ist dabei mit rot aufgetragen, da auf dieser Linie am Punkt $y=0 \mathrm{~mm}$ $M$ ermittelt wurde.

Bei der $\tilde{u}$-Komponente ist zu erkennen, dass eine Struktur, die sich gleich an der Hinterkante auf der Hauptströmungsseite befindet, mit steigender $x$-Koordinate allmählich kleiner wird. $\tilde{u}_{\text {All }}^{\text {Kom }}$ ist etwa im Bereich $20 \mathrm{~mm} \leq x \leq 40 \mathrm{~mm}$ minimal und wird ab $x \geq 40 \mathrm{~mm}$ insbesondere in der Scherschicht größer. Die $\tilde{v}$-Komponente hat auch eine signifikante Struktur in unmittelbarer Nähe der Hinterkante, die sich aber nicht wie bei $\tilde{u}_{A l l}^{\text {Kom }}$ weiter in Strömungsrichtung ausdehnt. $|\tilde{v}|$ wird wie $|\tilde{u}|$ im Bereich $x \geq 40 \mathrm{~mm}$ ebenfalls deutlich größer. Details der hier gesehenen Struktur werden im nächsten Abschnitt weiter diskutiert.

Die Abbildung 5.3 zeigt die Profile $\tilde{u}_{A l l}^{\text {Kom }}$ und $\tilde{v}_{A l l}^{\text {Kom }}$ bei $x=60 \mathrm{~mm}$ im Vergleich mit der durch die Schwingkante allein erzeugten Wechselgeschwindigkeit $\tilde{u}_{A l l}^{K l}$ und $\tilde{v}_{A l l}^{K l}$ (oben ist die $\tilde{u}$ - und unten die $\tilde{v}$-Komponente dargestellt). Da $\left|\tilde{u}_{A l l}^{\text {Kom }}\right|,\left|\tilde{v}_{A l l}^{\text {Kom }}\right|$ nur ca. $10 \%$ der Amplitude von $\tilde{u}_{A l l}^{K l}$ und $\tilde{v}_{A l l}^{K l}$ betragen, und vor allem die Messungenauigkeit von $\tilde{u}_{A l l}^{K o m}$ und $\tilde{v}_{A l l}^{K o m}$ so groß wie die Werte selbst ist, kann man davon ausgehen, dass die vorhandenen zur Anregung kohärenten kleinen Messwerte auf eine unzureichende Genauigkeit bei der Kompensation zurückzuführen sind. 

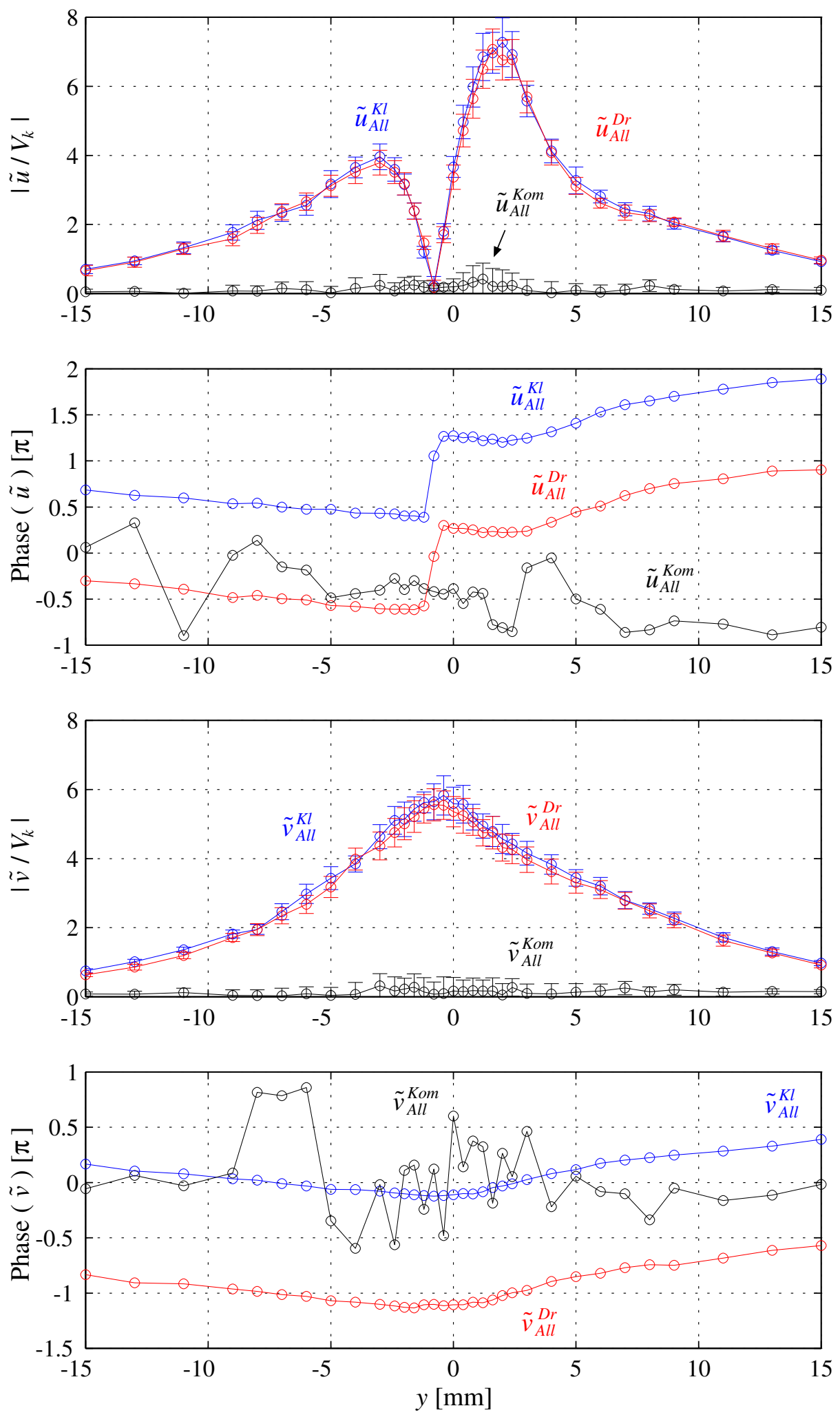

Abb. 5.1: Wechselgeschwindigkeiten der Kompensationsfelder und der durch die Schwingkanten-Anregung sowie durch Druckgradienten-Anregung erzeugten Felder bei $x=30 \mathrm{~mm}$ für $1.6 \mathrm{~Hz}$ : Betrag (a) und Phase (b) der $\tilde{u}$-Komponente, Betrag (c) und Phase (d) der $\tilde{v}$-Komponente 

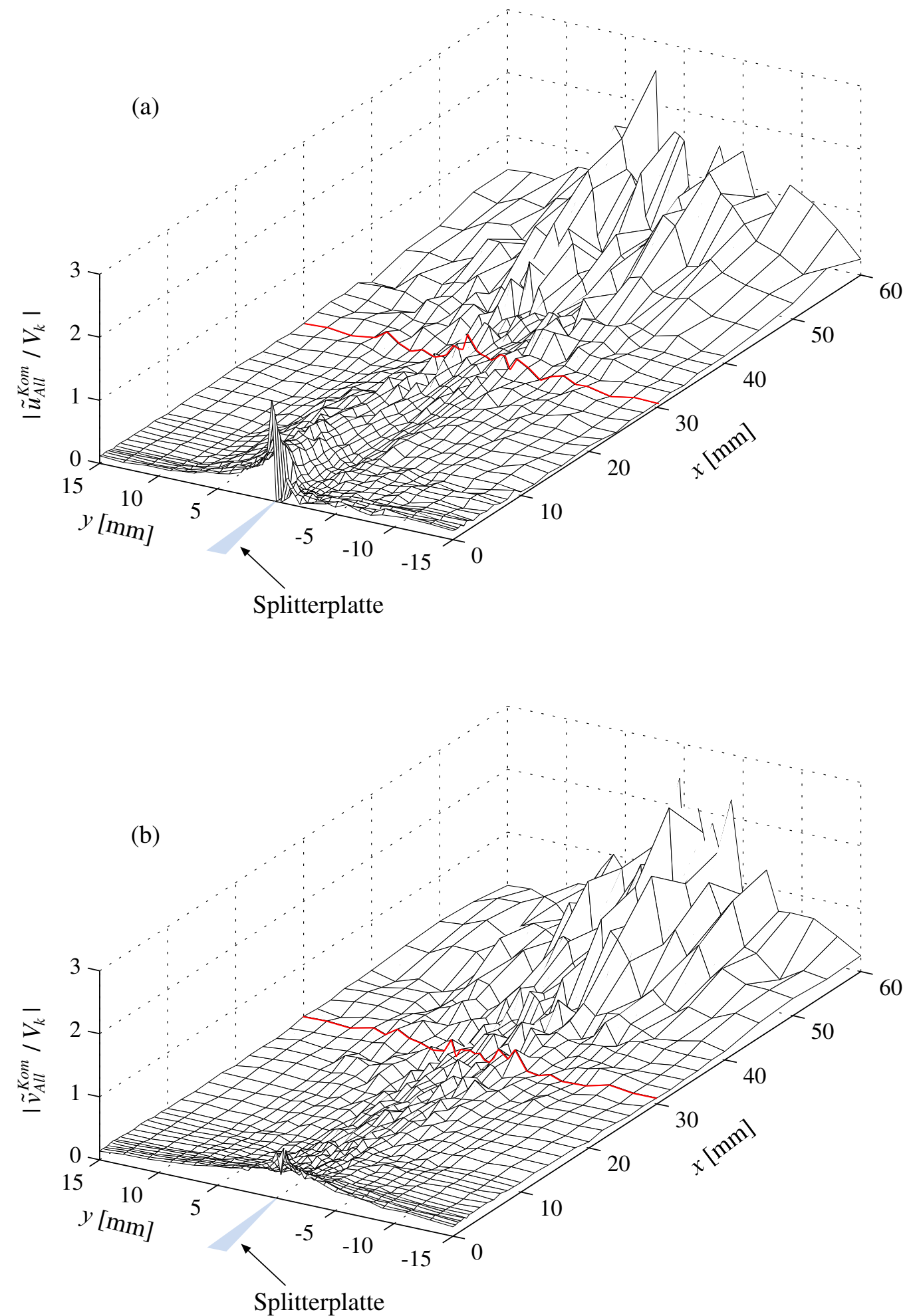

Abb. 5.2: Übersicht über den Betrag der Wechselgeschwindigkeit im Kompensationsfeld bei $1.6 \mathrm{~Hz}$ : oben die $\tilde{u}$ - und unten die $\tilde{v}$-Komponente 

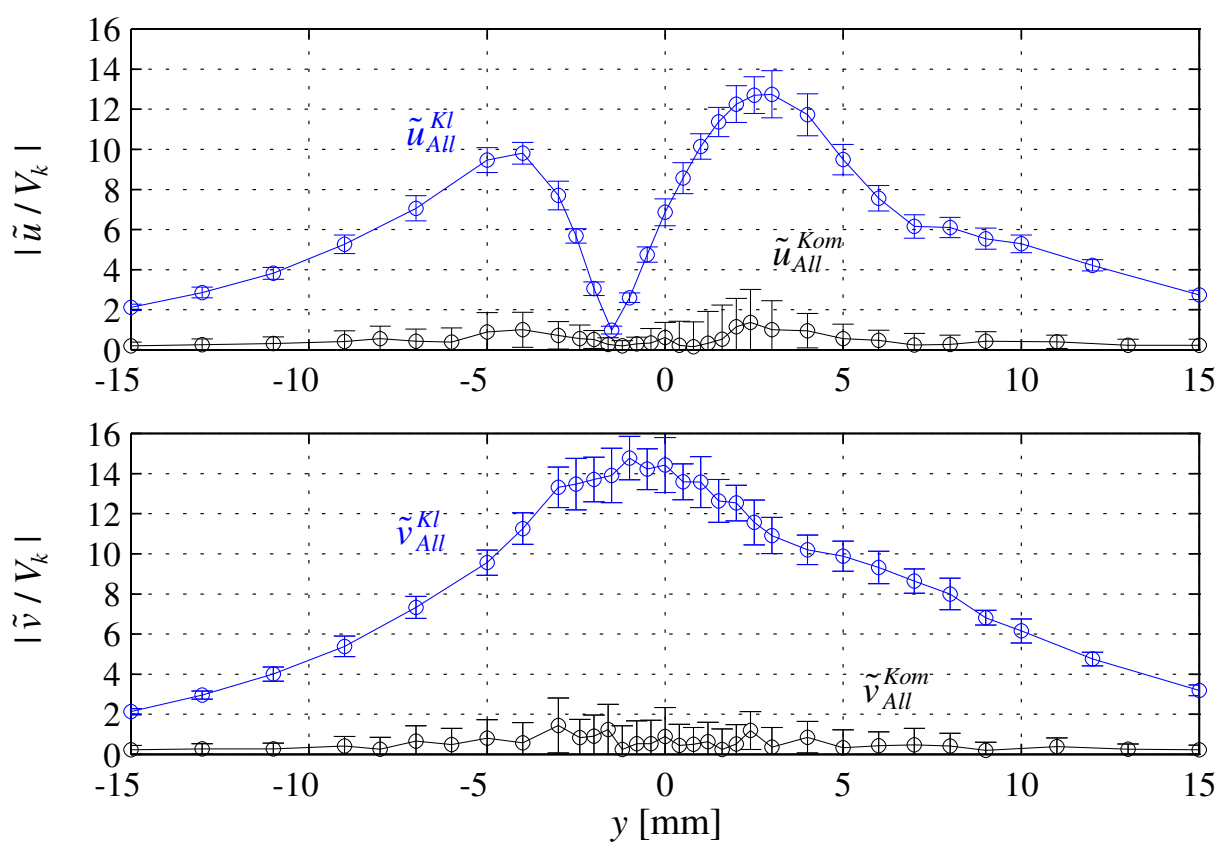

Abb. 5.3: Betrag der Wechselgeschwindigkeiten des Kompensationsfeldes und des durch Schwingkanten-Anregung erzeugten Feldes bei $x=60 \mathrm{~mm}$ für $1.6 \mathrm{~Hz}$ : oben die $\tilde{u}$ - und unten die $\tilde{v}$-Komponente

Zusammenfassend kann aus den in den Abbildungen 5.1 bis 5.3 gezeigten Ergebnissen gefolgert werden, dass das durch die Schwingkanten- oder die DruckgradientenAnregung erzeugte Fernfeld nur durch einen einzigen Freiheitsgrad, also durch die angefachte Mode der KH-Welle, beschrieben werden kann. Man kann somit erwarten, dass die angefachte Lösung der KH-Wellen im Kompensationsfeld nicht mehr vorhanden ist. Das Kompensationsfeld wird somit wie folgt zerlegt werden:

$$
\tilde{\psi}_{A l l}^{K o m}=\tilde{\psi}_{A n F}^{K o m}+\underbrace{\tilde{\psi}_{K H_{A}}^{K o m}}_{=0}+\tilde{\psi}_{K H_{D}}^{K o m}+\tilde{\psi}_{N F}^{K o m}
$$

Obwohl angenommen wird, dass die angefachte Mode der KH-Wellen im Kompensationsfeld verschwunden ist, soll hier bemerkt werden, dass die gedämpften Mode $\tilde{\psi}_{K H_{D}}^{K o m}$ noch in diesem Feld vorhanden ist.

\subsubsection{Die Kompensation in Abhängigkeit von der Anregungs- frequenz}

Beide Komponenten der Wechselgeschwindigkeiten im Kompensationsfeld weisen an dem Ort ein Minimum auf, wo $M$ ermittelt wurde. Es stellt sich trotzdem die Frage, ob der Ort der Kompensation mit $x=30 \mathrm{~mm}$ bei $1.6 \mathrm{~Hz}$ wirklich in dem Bereich liegt, in dem das durch die Schwingkante angeregte Feld allein durch die angefachte Mode der 
Kapitel 5. Untersuchung der Nahfelder mittels Kompensationsexperiment

KH-Wellen beschrieben wird. Das bedeutet, dass dort nicht nur $\tilde{\psi}_{A n F}^{K l}$ sondern auch $\tilde{\psi}_{N F}^{K l}$ sowie $\tilde{\psi}_{K H_{D}}^{K l}$ klein gegen $\tilde{\psi}_{K H_{A}}^{K l}$ sein sollten. Da der Bereich, in dem die angefachte KHWelle voll entwickelt ist, von ihrer Wellenlänge abhängt, hängt somit die $x$-Koordinate des optimalen Kompensationsortes von der Anregungsfrequenz ab.

Für die weitere Diskussion wird hier ein Kompensationskoeffizient $K$ durch

$$
K(x, f):=\frac{V_{k}(f)}{V_{m}(x, f)}
$$

definiert, der von der Frequenz und der $x$-Koordinate abhängt. $V_{m}$ kennzeichnet die durch die Normierungsgröße $V_{n}$ charakterisierte Anregungsamplitude, die die Kompensationsamplitude $M$ schon enthält. Da $M$ vom Kompensationsort abhängt, hängt somit $V_{m}$ auch von der $x$-Koordinate ab. $K$ ist in unserem Fall ein im Vergleich zu $M$ geeigneterer Kompensationskoeffizient, da sich $K$ auf die Wechselgeschwindigkeiten der beiden Anregungsvorrichtungen bezieht, während $M$ nur eine auf die Stromfunktion bezogene Größe ist.

Abbildung 5.4 (a) zeigt $K$ in der komplexen Ebene. Der Kompensationsort wurde dabei für sechs verschiedenen Frequenzen $(0.8 \mathrm{~Hz}, 1.0 \mathrm{~Hz}, 1.2 \mathrm{~Hz}, 1.6 \mathrm{~Hz}, 2.0 \mathrm{~Hz}$ und $2.4 \mathrm{~Hz}) \mathrm{im}$ Bereich $0<x \leq 70 \mathrm{~mm}$ variiert, da noch weiter stromab der Hinterkante aufgrund von dort auftretenden nichtlinearen Effekten der lineare Ansatz der Kompensation nicht mehr verwendet werden kann.

Es ist zu erkennen, dass sich der Koeffizient $K$ für alle Frequenzen mit steigender $x$ Koordinate einem etwa konstanten Wert annähert, wobei er allerdings bei den tiefen Frequenzen für große $x$ stark streut. Man kann damit feststellen, dass Orte, zwischen denen sich $K$ nicht mehr systematisch ändert, als Kompensationsorte $x_{K}$ geeignet sind. Der endgültige Kompensationskoeffizient $\bar{K}(f)$ wurde nun durch die Mittelung der in Abbildung 5.4 (a) gezeigten letzten vier Punkte bestimmt, da die Werte dort nur noch stochastisch streuen.

Abbildung 5.4 (b) zeigt die Amplitude von $\bar{K}(\ln |\bar{K}|=\operatorname{Re}\{\ln \bar{K}\}$ : blaue Punkte) und die Phasenverschiebung zwischen den beiden Anregungen $\Delta \bar{\varphi}_{K}=\operatorname{Im}\{\ln \bar{K}\}$ (rote Punkte) in Abhängigkeit von der Anregungsfrequenz $f=\beta / 2 \pi$. Ein Voreilen der Phase von $V_{m}$ gegenüber der von $V_{k}$ entspricht einem negativen $\Delta \bar{\varphi}_{K}$. Da die für die Kompensation nötige Verzögerungszeit $\Delta T_{K}$ der Druckgradienten-Anregung gegenüber der Schwingkanten-Anregung durch $\Delta \bar{\varphi}_{K}(\beta)$ beschrieben wird $\left(\Delta T_{K}=d \Delta \bar{\varphi}_{K} / d \beta\right)$, ist $\Delta T_{K}$ (schwarze Linie) auch in der Abbildung aufgetragen. $\Delta T_{K}$ wurde dabei durch Anpassung eines Polynom zweites Grades (rote Kurve) an $\Delta \bar{\varphi}_{K}$ bestimmt.

Man sieht aus der Darstellung, dass die Amplitude von $\bar{K}$ mit steigender Frequenz zunimmt. Das bedeutet, dass die Effektivität der Druckgradienten-Anregung bezogen auf die der Schwingkanten-Anregung mit der Frequenz ansteigt. Mit anderen Worten: die Schwingkante regt bei tiefen Frequenzen die Instabilitätswellen effektiver an als der durch die Membranen erzeugte Druckgradient. Dagegen reagiert die KH-Welle im Bereich etwa $f<1.8 \mathrm{~Hz}$ schneller auf die Druckgradienten-Anregung als auf die Schwingkanten-Anregung $\left(\Delta T_{K}>0\right)$.

Die Ergebnisse legen die Vermutung nahe, dass die Schwingkante zwei verschiedene Anregungsmechanismen bewirkt. Einer ist die Ausgleichströmung, d.h. die durch die 


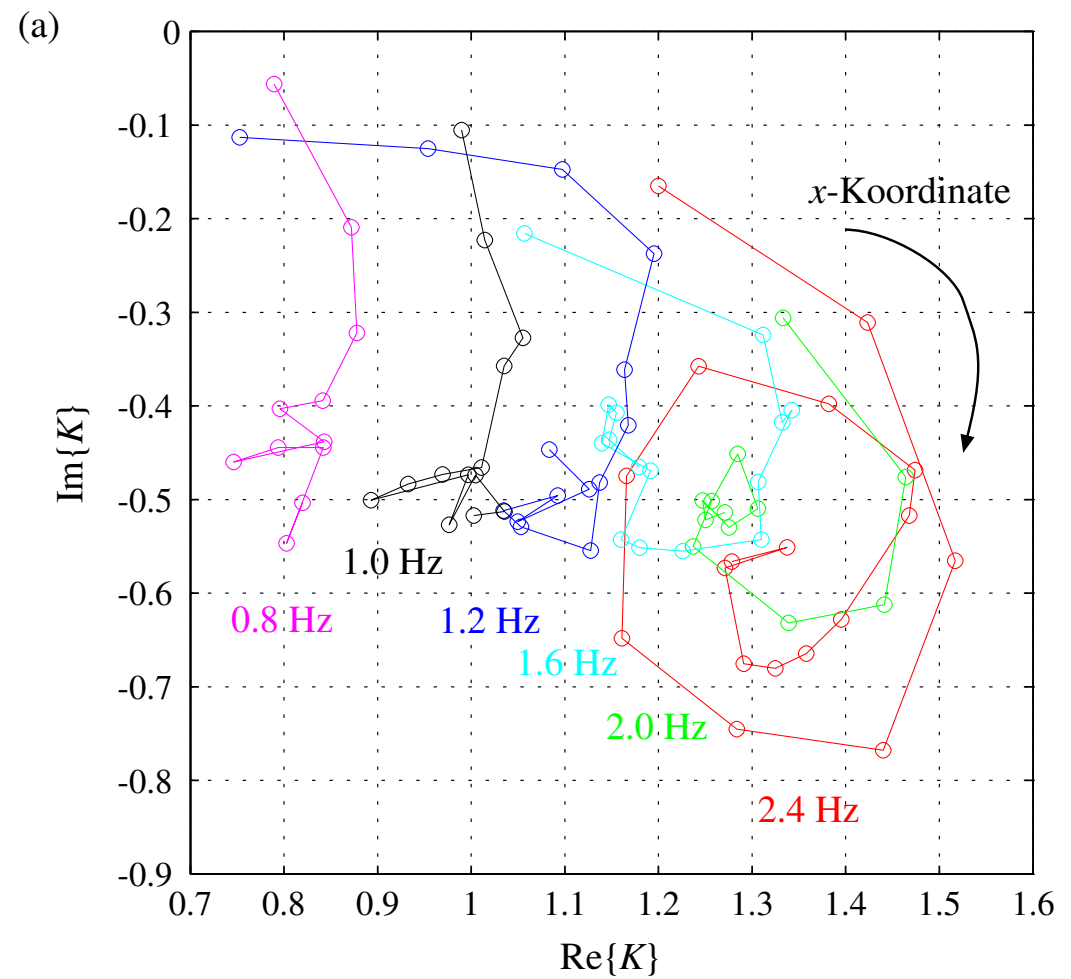

(b)

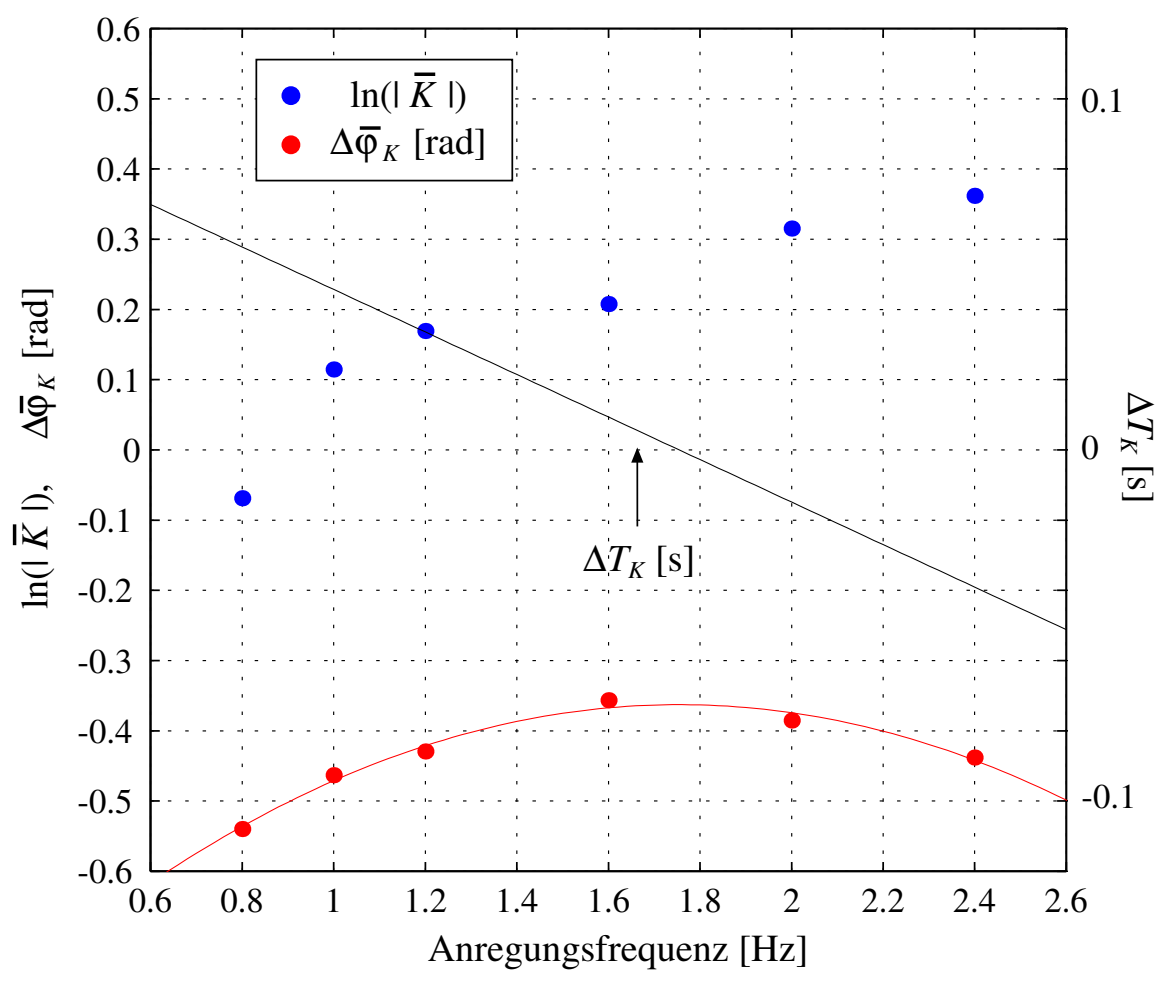

Abb. 5.4: (a) Der Kompensationsparameter $K$ in Abhängigkeit von der $x$-Koordinate für die verschiedenen Anregungusfrequenzen in der komplexen Ebene, (b) Abhängigkeit der Kompensationsparameter $|\bar{K}|$ und $\Delta \bar{\varphi}_{K}$ sowie $\Delta T_{K}$ von der Anregungsfrequenz 
Kapitel 5. Untersuchung der Nahfelder mittels Kompensationsexperiment

Schwingung der Hinterkante zustande gekommene Potenzialströmung (eine solche Potenzialströmung spielt auch bei der Druckgradienten-Anregung die Hauptrolle).

Der zweite Anregungsmechanismus basiert auf der Auslenkung der Schwingkante, die zur Verschiebung des $\bar{u}$-Profils in $y$-Richtung in unmittelbarer Nähe der Hinterkante führt. Bei tiefen Frequenzen, wie im Abschnitt 4.1.2 erwähnt, wird die Strömung gemäß der Kutta-Bedingung gezwungen, die $\tilde{v}$-Komponente an der feststehenden Hinterkante (Druckgradienten-Anregung) zu unterdrücken. Diese Wirkung steht vermutlich, wie anhand der Abbildungen 4.22 und 4.23 diskutiert wurde, mit der Amplitude der KHWellen in einem engen Zusammenhang. Da die Druckgradienten-Anregung mit abnehmender Frequenz aufgrund der Kutta-Bedingung immer weniger die $\tilde{v}$-Komponente bzw. die Auslenkung der Scherschicht an der Hinterkante erzeugen kann, und diese bei der Schwingkanten-Anregung dagegen wegen der Bewegung der Hinterkante an dieser Stelle immer vorhanden ist, ist die Schwingkanten-Anregung bei tiefen Frequenzen effektiver als die Druckgradienten-Anregung. Es ist somit anzunehmen, dass bei der Schwingkanten-Anregung die KH-Instabilität bei tiefen Frequenzen vornehmlich durch die Grenzschichtauslenkung, bei hohen Frequenzen durch die Ausgleichsströmung angeregt wird.

\subsection{Das nichtanregende Nahfeld}

Das Kompensationsfeld $\tilde{\psi}_{\text {All }}^{\text {Kom }}$, das gemäß Gleichung (5.2) aus den drei dort genannten Teilfeldern besteht, hat, wie in Abbildung $5.2 \mathrm{zu}$ sehen war, im Bereich nahe der Hinterkante eine deutliche Struktur. Um die Wechselwirkung zwischen der Gleichströmung und dem anregenden Feld im Kompensationsfeld zu untersuchen, wird zuerst das zusammengesetzte anregende Feld $\tilde{\psi}_{A n F}^{K o m}$, das durch die Überlagerung der beiden anregenden Felder entsteht $\left(\tilde{\psi}_{A n F}^{K o m}=\tilde{\psi}_{A n F}^{K l}+M \cdot \tilde{\psi}_{A n F}^{D r}\right)$, von $\tilde{\psi}_{A l l}^{K o m}$ abgezogen:

$$
\tilde{\psi}_{K H_{D}+N F}^{K o m}:=\tilde{\psi}_{A l l}^{K o m}-\tilde{\psi}_{A n F}^{K o m}=\tilde{\psi}_{K H_{D}}^{K o m}+\tilde{\psi}_{N F}^{K o m}
$$

Abbildung 5.5 zeigt das Kompensationsfeld ohne das anregende Feld $\tilde{\psi}_{K H_{D}+N F}^{K o m} \mathrm{im}$ Bereich nahe der Hinterkante bei $1.6 \mathrm{~Hz}$ (der mit $V_{k}$ normierte Betrag (a) der $\tilde{u}^{-}$ Komponente und (b) der $\tilde{v}$-Komponente). Bei der $\tilde{u}$-Komponente ist zu erkennen, dass die auf der Hauptströmungsseite vorhandene Struktur eine $\partial \bar{u} / \partial y$-ähnliche Form erhält, die glatt von stromauf der Hinterkante in die Scherschicht übergeht. Im Nebenströmungsbereich ist dagegen kein nennenswerter Betrag zu sehen. Die $\tilde{v}$ - ist deutlich kleiner als die $\tilde{u}$-Komponente und zeigt einen „Peak“ in unmittelbarer Nähe der Hinterkante. Im Hauptströmungsbereich stromauf der Hinterkante sieht man ebenfalls eine Struktur, deren Amplitude mit steigendem Wandabstand allmählich abnimmt. Wie sich diese Struktur in $x$-Richtung ändert, ist bei der Darstellung in Abbildung 5.5 nicht deutlich erkennbar. In der Nebenströmung ist sie ähnlich klein wie die $\tilde{u}$-Komponente. 


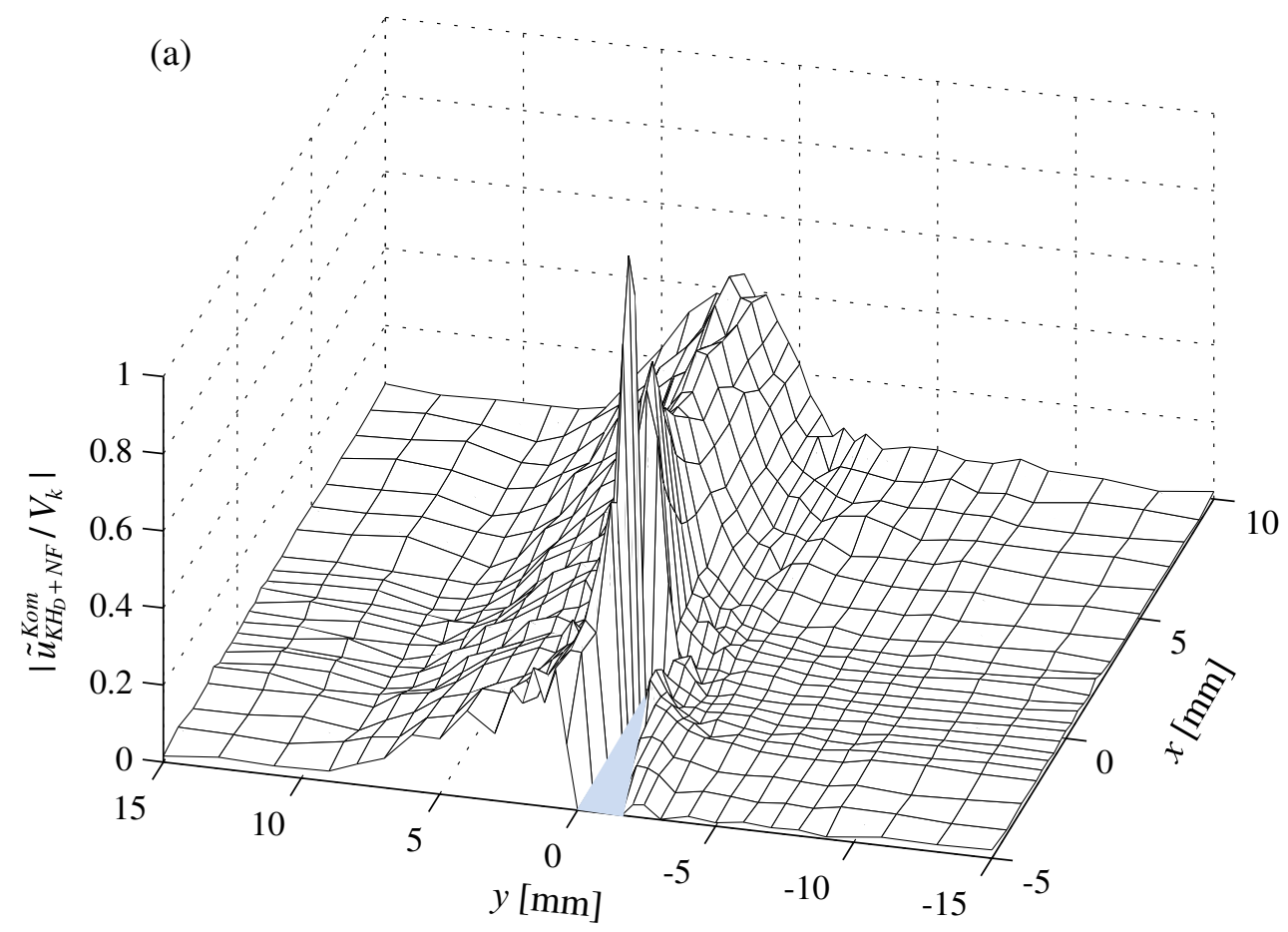

(b)

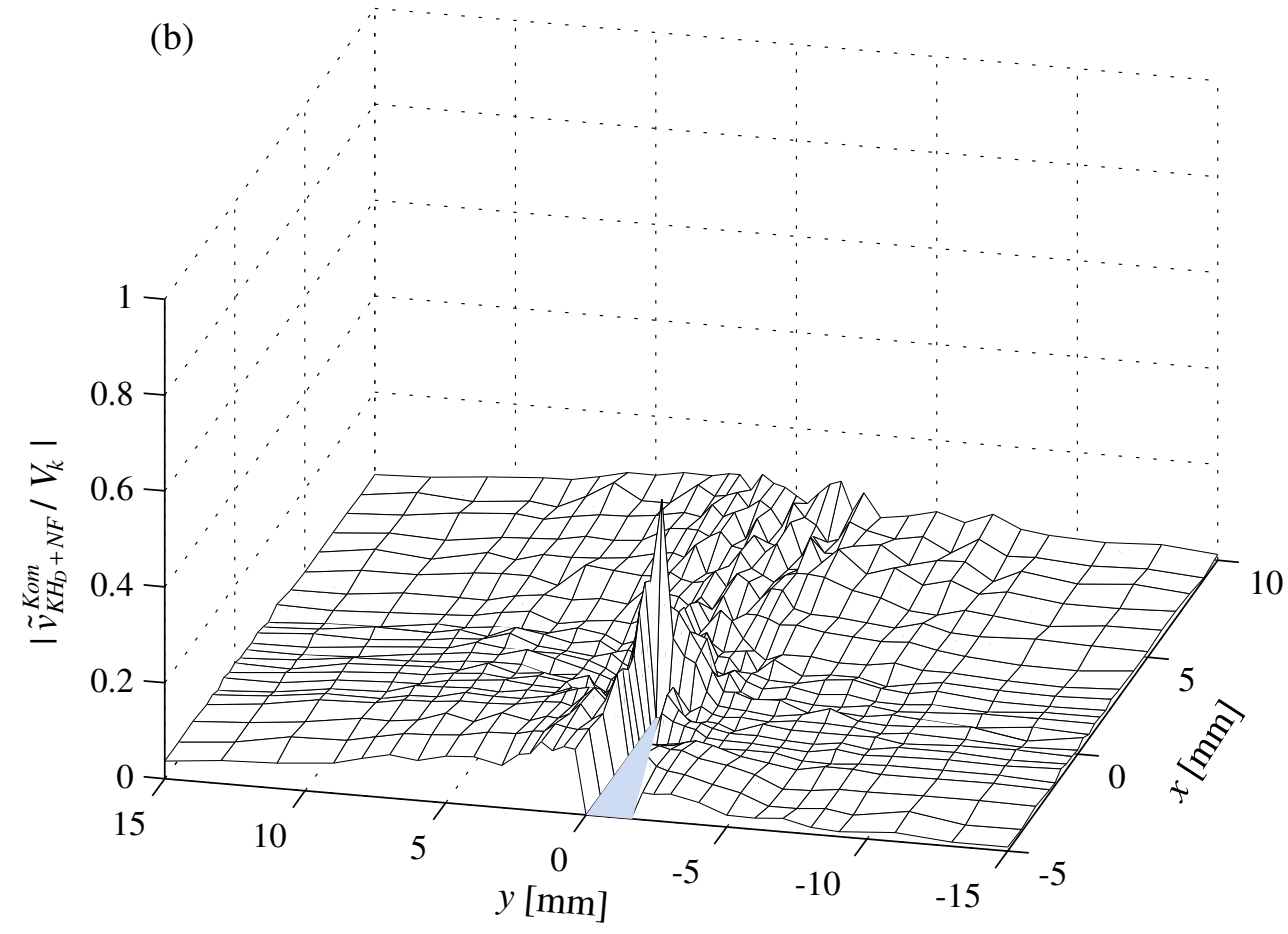

Abb. 5.5: Kompensationsfeld ohne das anregende Feld $\tilde{\psi}_{K H_{D}+N F}^{K o m}$ im Nahbereich der Hinterkante bei $1.6 \mathrm{~Hz}$ : der mit $V_{k}$ normierte Betrag der $\tilde{u}$-Komponente (a) und der $\tilde{v}$-Komponente (b) 


\subsubsection{Die angefachte und gedämpfte Mode der KH-Wellen im Kompensationsfeld}

Um das Nahfeld $\tilde{\psi}_{N F}^{K o m}$ von $\tilde{\psi}_{K H_{D}+N F}^{K o m}$ zu extrahieren, wird das Feld der gedämpften KHWellen im Kompensationsfeld durch den Ansatz (Gleichung (4.21)) bestimmt. Man erwartet, dass der Vorfaktor für die angefachte Lösung der KH-Wellen im Kompensationsfeld $C_{A}^{K o m}$ klein ist, während er für die gedämpfte Lösung $C_{D}^{K o m}$ einen nenneswerten Betrag haben kann.

Der Betrag und die Phase von $C_{A}^{K o m}$ bzw. $C_{D}^{K o m}$ sind in Abhängigkeit von der $x$ Koordinate in Abbildung 5.6 aufgetragen. Zum Vergleich wurden die für $\tilde{\psi}_{K H+N F}^{K l}$ bestimmten Vorfaktoren $C_{A}^{K l}$ bzw. $C_{D}^{K l}$ und die für $M \cdot \tilde{\psi}_{K H+N F}^{D r}$ bestimmten $C_{A}^{D r}$ bzw. $C_{D}^{D r}$ auch in der Abbildung dargestellt. Die Anregungsfrequenz ist dabei $1.6 \mathrm{~Hz}$.

Man sieht bei $C_{A}$, dass die durch die Schwingkante und durch den Druckgradienten angeregten angefachten Moden mit entgegengesetzten Phasenbeziehung sehr ähnlich verlaufen. Da einerseits die Beträge von ihnen für kleine $x$ etwas unterschiedlich sind, sie sich anderseits mit steigender $x$-Koordinate annähern, kann man davon ausgehen, dass zum einen der Unterschied des Anregungsmechanismus der beiden Vorrichtungen besonders nahe der Hinterkante erkennbar ist, zum anderen die durch diese beiden Vorrichtungen angeregten Instabilitätswellen sich im Fernfeld gegenseitig auslöschen: $C_{A}^{\text {Kom }}$ ist sehr klein.

Die im Zusammenhang mit Abbildung 4.23 erwähnte Vermutung war, dass in einem Feld mit angeregten Instabilitätswellen die angefachte Mode in die gedämpfte Mode gestreut wird. Dies führt dazu, dass der Vorfaktor für die gedämpfte Mode $C_{D}$ mit steigender $x$-Koordinate anwächst. In Abbildung 5.6 (c) ist zu erkennen, dass sowohl $C_{D}^{K l}$ als auch $C_{D}^{D r}$ mit steigender $x$-Koordinate zunimmt. Im Kompensationsfeld bleibt dagegen $C_{D}^{K o m}$ für $x>3 \mathrm{~mm}$ nahezu konstant, da in diesem Feld die angefachte Mode nicht vorhanden ist. Die Vermutung einer Wechselwirkung zwischen den beiden Moden lässt sich somit erhärten. Zum Vergleich wurde die Summe der beiden Vorfaktoren $C_{D}^{K l D r}=C_{D}^{K l}+C_{D}^{D r}$ in der Abbildung mit grüner Farbe dargestellt. Man stellt aus dem Vergleich zwischen $C_{D}^{K o m}$ und $C_{D}^{K l D r}$ fest, dass $\tilde{\psi}_{K H_{D}}^{K o m}$ nicht durch die Überlagerung zwischen $\tilde{\psi}_{K H_{D}}^{K l}$ und $M \cdot \tilde{\psi}_{K H_{D}}^{D r}$ beschrieben werden kann. Der Verlauf von $C_{D}^{K o m}$ bedeutet auch, dass im Kompensationsfeld die Abhängigkeit der gedämpften Mode von der $x$ Koordinate für großes $x$ allein durch die lokale Wellenzahl bestimmt wird, also die gedämpfte Lösung der KH-Wellen außerhalb des Kantennahebereichs mit steigender $x$-Koordinate exponentiell abnimmt. Was noch auffällt ist, dass die Schwingkante im aufgetragenen Bereich die gedämpfte Mode mit kleinerer Amplitude anregt als der Druckgradient, während die beiden Anregungsvorrichtungen im Fernfeld die gleiche angefachte Mode anregen.

\subsubsection{Die lineare Beschreibung des Nahfeldes}

Die Frage an dieser Stelle ist, ob das Nahfeld im Kompensationsfeld $\tilde{\psi}_{N F}^{K o m}$ als eine lineare Überlagerung der beiden Nahfelder $\tilde{\psi}_{N F}^{K l}$ und $\tilde{\psi}_{N F}^{D r}$ angesehen werden kann. Da die Nahfelder $\tilde{\psi}_{N F}^{K l}$ und $\tilde{\psi}_{N F}^{D r}$ gemäß Gleichung (4.5) und (4.21) bestimmt werden 

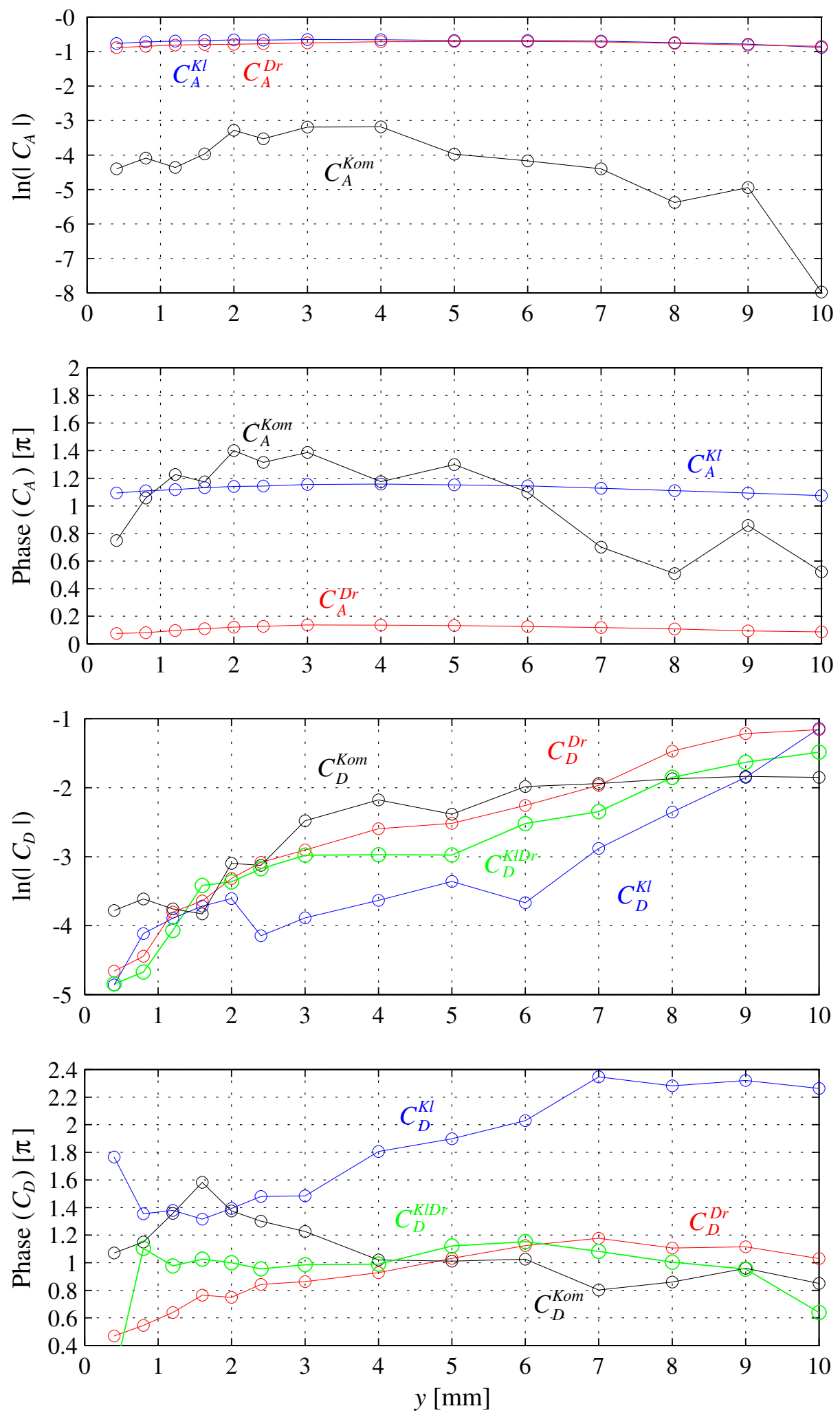

Abb. 5.6: Die durch den Ansatz (Gleichung (4.21)) bestimmten Vorfaktoren $C_{A}$ und $C_{D}$ im Kompensationsfeld bei $1.6 \mathrm{~Hz}$ : (a) der Betrag von $C_{A}$, (b) die Phase von $C_{A}$, (c) der Betrag von $C_{D}$ und (d) die Phase von $C_{D}$ 
Kapitel 5. Untersuchung der Nahfelder mittels Kompensationsexperiment

können, stehen für die Untersuchung dieser Frage sowohl die Überlagerung dieser beiden primären Nahfelder als auch das kompensierte Nahfeld $\tilde{\psi}_{N F}^{K o m}$ zur Verfügung,

$$
\tilde{\psi}_{N F}^{K l D r}:=\tilde{\psi}_{N F}^{K l}+M \cdot \tilde{\psi}_{N F}^{D r}
$$

das mit dem oben bestimmten $C_{D}^{K o m}$ aus $\tilde{\psi}_{K H_{D}+N F}^{K o m}$ extrahiert werden und mit $\tilde{\psi}_{N F}^{K l D r}$ verglichen werden kann. Die Abbildungen 5.7 und 5.8 zeigen den Vergleich zwischen den Beträgen der $\tilde{u}$ - und der $\tilde{v}$-Komponente in einer dreidimensionalen Darstellung bei 1.6 Hz. Oben ist das Nahfeld im Komensationsexperiment, unten die Überlagerung von $\tilde{\psi}_{N F}^{K l}$ und $\tilde{\psi}_{N F}^{D r}$ dargestellt. Um die einzelnen Profile der $\tilde{u}$ - und $\tilde{v}$-Komponente besser miteinander vergleichen zu können, sind sie bei $x=0.4 \mathrm{~mm}, x=4 \mathrm{~mm}$ und $x=8 \mathrm{~mm}$ in Abbildung 5.9 bis 5.11 aufgetragen. Die blauen Kurven entsprechen dem Nahfeld im Kompensationsexperiment und die roten Kurven dem überlagerten Nahfeld.

Die dreidimensionalen Darstellungen in den Abbildungen 5.7 und 5.8 zeigen, dass die beiden Nahfelder eine sehr ähnliche Struktur, besonders bei der $\tilde{u}$-Komponente, haben, während die $\tilde{v}$-Komponente deutlich kleiner ist als die $\tilde{u}$-Komponente, und der genaue Unterschied zwischen den beiden $\tilde{v}$-Komponenten bei der Darstellung nicht zu sehen ist. Was man noch bei der Darstellung bedenken sollte, ist, dass die hier gesehene Struktur etwa bei $x=20 \mathrm{~mm}$ verschwinden muss (siehe Abbildung 5.2).

Vergleicht man die beiden Profile bei $x=0.4 \mathrm{~mm}$ (siehe Abbildung 5.9), also dicht stromab der Hinterkante, so ist noch deutlicher zu erkennen, dass sich die verbliebende Struktur hauptsächlich im Hauptströmungsbereich befindet und in diesem Bereich das überlargerte Nahfeld $\left(\tilde{u}_{N F}^{K l D r}\right.$ bzw. $\left.\tilde{v}_{N F}^{K l D r}\right)$ und das Nahfeld im Kompensationsexperiment $\left(\tilde{u}_{N F}^{K o m}\right.$ bzw. $\left.\tilde{v}_{N F}^{K o m}\right)$ sehr gut übereinstimmen. Im Bereich $y \lesssim-2 \mathrm{~mm}$ sind die Beträge der Wechselgeschwindigkeitskomponenten sehr klein, und somit streuen die dazugehörigen Phasen unsystematisch. Da die $\tilde{v}$-Komponente im Hauptströmungsbereich einen fast konstanten Wert hat, der größer ist als der im Nebenströmungsbereich, kann sie nicht als ein symmetrisches Profil angesehen werden.

$4 \mathrm{~mm}$ weiter stromab der Hinterkante (Abbildung 5.10) ist das Maximum der $\tilde{u}^{-}$ Komponente kleiner und das Profil in $y$-Richtung breiter geworden. Dieses Ergebnis deutet darauf hin, dass die Änderung des Profils $\left(\tilde{u}_{N F}^{K l D r}\right.$ bzw. $\left.\tilde{u}_{N F}^{K o m}\right)$ in $x$-Richtung durch die Inhomogenität der Gleichströmung zustande gekommen sein könnte (eine ähnliche Änderung der Struktur ist bei der Darstellung der Gleichwirbeligkeit in der Abbildung $3.8 \mathrm{zu}$ sehen). Der „Peak" bei der v-Komponente ist hier verschwunden, während der konstante kleine Betrag im Hauptströmungsbereich fast unverändert geblieben ist. Von $x=4 \mathrm{~mm}$ bis $x=8 \mathrm{~mm}$ ändern sich die beiden Nahfelder nicht mehr so stark (siehe Abbildung 5.11). Es ist allerdings zu sehen, dass das Maximum der $\tilde{u}$-Komponente noch kleiner geworden ist, und vor allem der Betrag der $\tilde{v}$-Komponente für etwa $y>3 \mathrm{~mm}$ wieder zugenommen hat.

Aufgrund des Vergleichs der beiden Nahfelder bei den verschiedenen $x$-Koordinaten kann man davon ausgehen, dass $\tilde{\psi}_{N F}^{K l D r}$ und $\tilde{\psi}_{N F}^{K o m}$ sehr gut miteinander übereinstimmen. Das unterstützt die zugrunde liegende Annahme, dass das hier durch die Gleichung (4.5) definierte Nahfeld aus dem Gesamtfeld durch Subtraktion der KH-Wellen (Gleichung (4.21), die die Inhomogenität der Gleichströmung berücksichtigt) bestimmt werden kann, und insbesondere dass das Nahfeld linear beschrieben werden kann. Das 

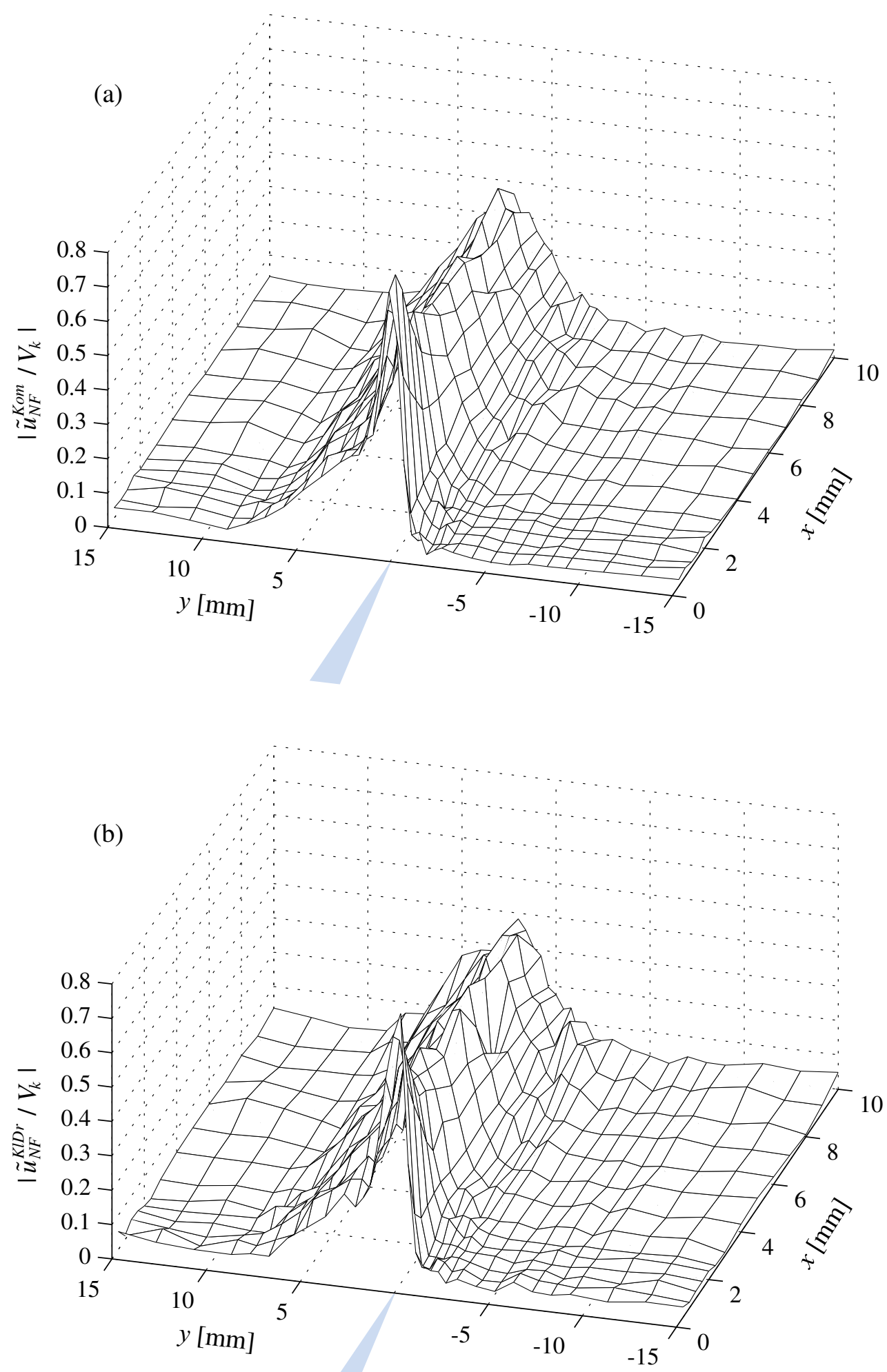

Abb. 5.7: Vergleich des Nahfeldes im Kompensationsexperiment $\tilde{\psi}_{N F}^{K o m}$ (a) mit dem durch Überlagerung von $\tilde{\psi}_{N F}^{K l}$ und $\tilde{\psi}_{N F}^{D r}$ bestimmten Nahfeld $\tilde{\psi}_{N F}^{K l D r}$ (b) bei $1.6 \mathrm{~Hz}$ : der mit $V_{k}$ normierte Betrag der $\tilde{u}$-Komponente 

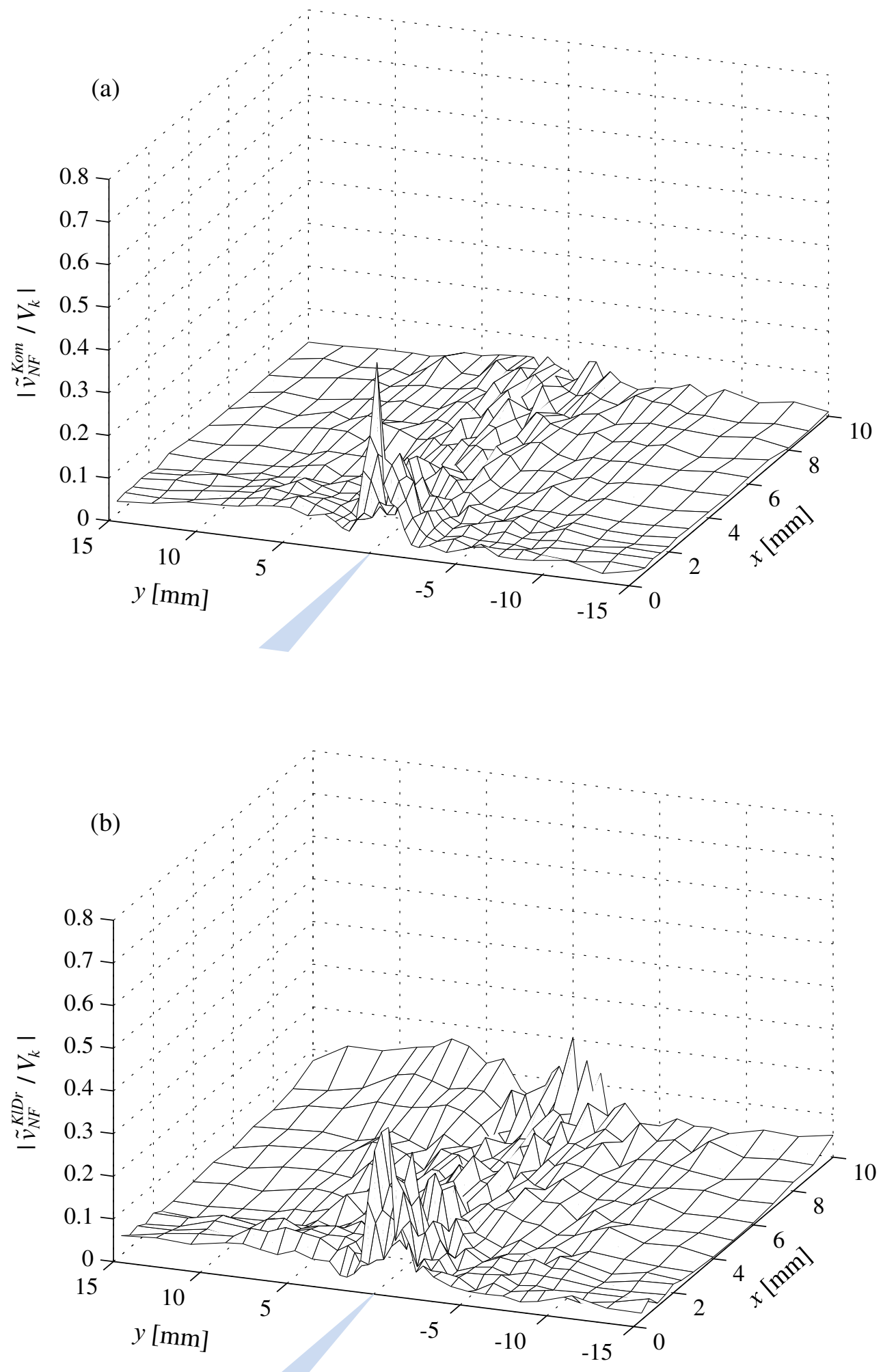

Abb. 5.8: Vergleich des Nahfeldes im Kompensationsexperiment $\tilde{\psi}_{N F}^{K o m}$ (a) mit dem durch die Überlagerung von $\tilde{\psi}_{N F}^{K l}$ und $\tilde{\psi}_{N F}^{D r}$ bestimmten Nahfeld $\tilde{\psi}_{N F}^{K l D r}$ (b) bei $1.6 \mathrm{~Hz}$ : der mit $V_{k}$ normierte Betrag der $\tilde{v}$-Komponente 

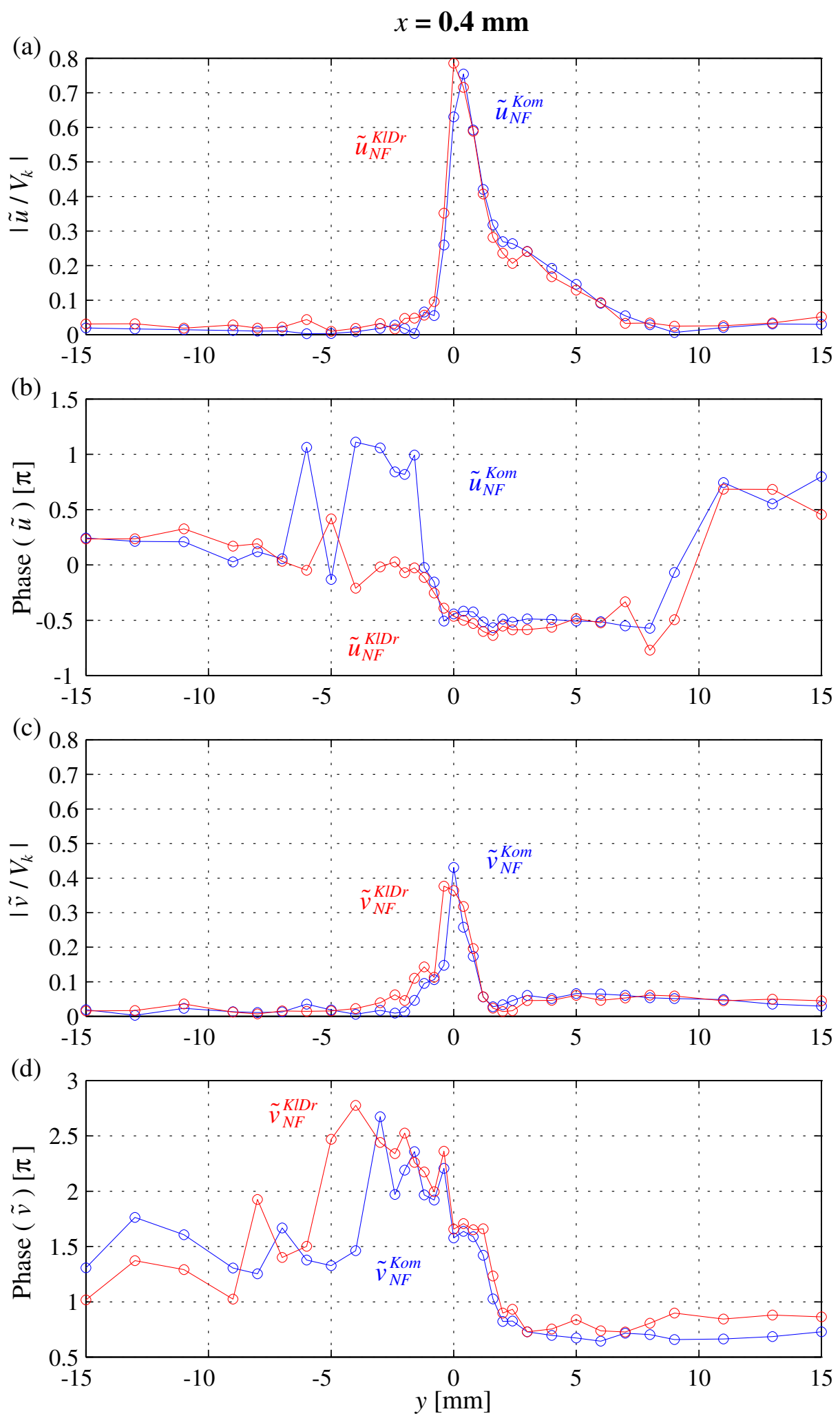

Abb. 5.9: Vergleich der Nahfelder im Kompensationsexperiment $\tilde{\psi}_{N F}^{K o m}$ (blaue Kurve) und bei Überlagerung von $\tilde{\psi}_{N F}^{K l}+\tilde{\psi}_{N F}^{D r}=\tilde{\psi}_{N F}^{K l D r}$ (rote Kurve) bei $x=0.4 \mathrm{~mm}$ für $1.6 \mathrm{~Hz}$ : (a) und (b): Betrag und Phase der normierten $\tilde{u}$-Komponente (c) und (d): Betrag und Phase der normierten $\tilde{v}$-Komponente 


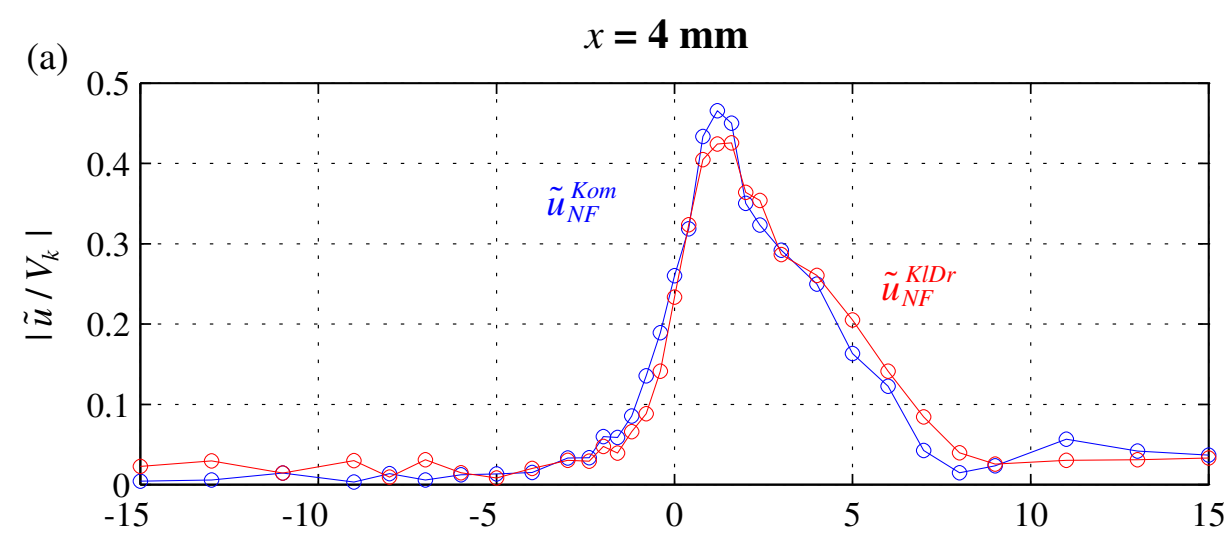

(b)

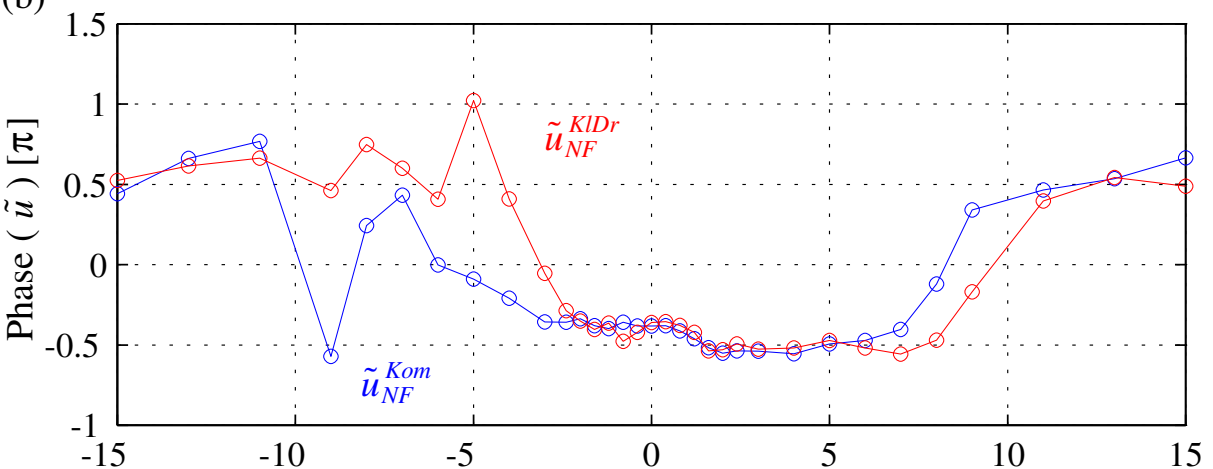

(c)

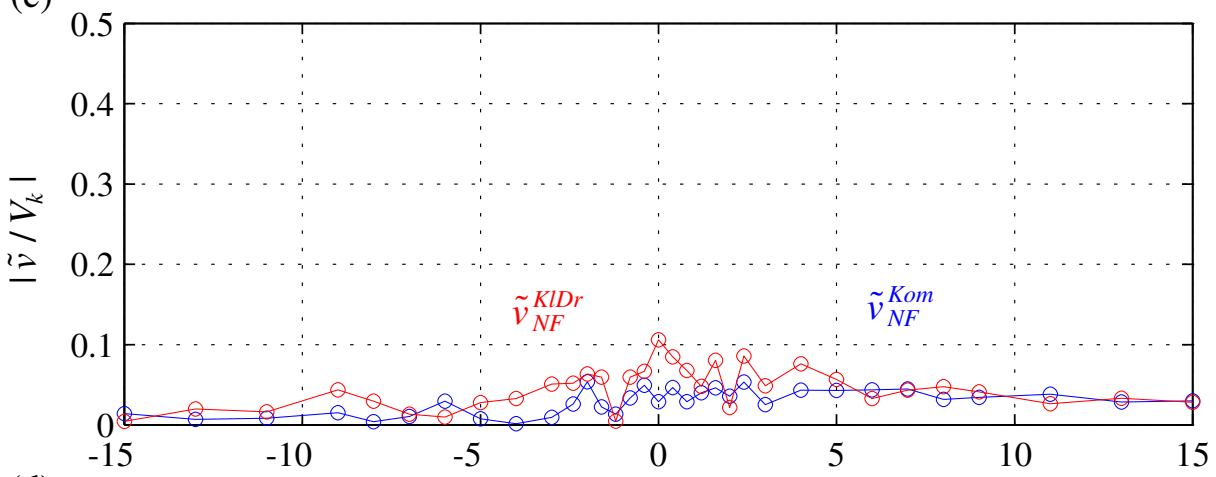

(d)

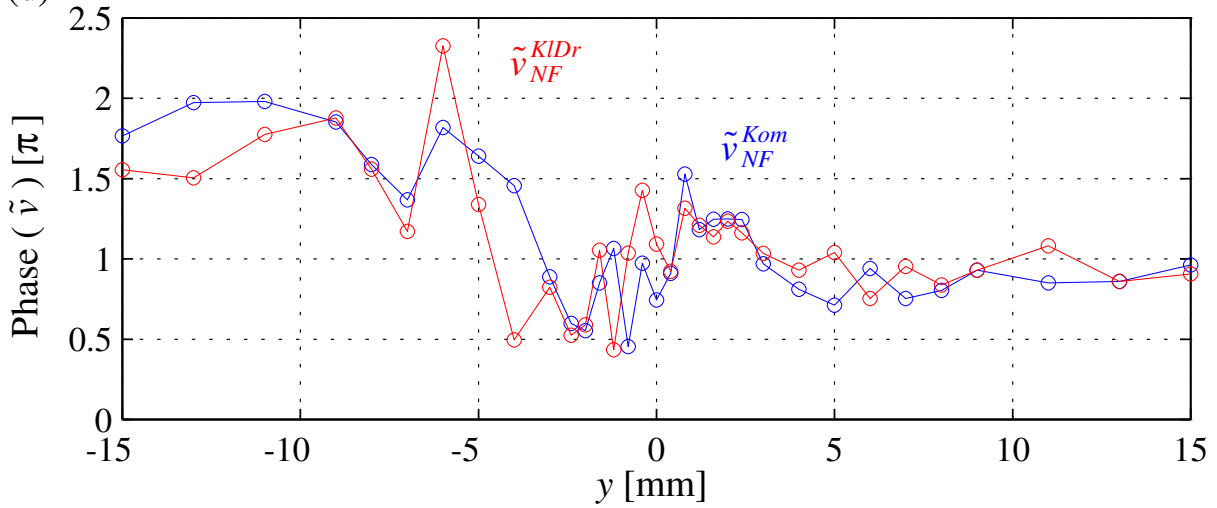

Abb. 5.10: Vergleich der Nahfelder im Kompensationsexperiment $\tilde{\psi}_{N F}^{\text {Kom }}$ (blaue Kurve) und bei Überlagerung von $\tilde{\psi}_{N F}^{K l}+\tilde{\psi}_{N F}^{D r}=\tilde{\psi}_{N F}^{K l D r}$ (rote Kurve) bei $x=4 \mathrm{~mm}$ für $1.6 \mathrm{~Hz}$ : (a) und (b): Betrag und Phase der normierten $\tilde{u}$-Komponente (c) und (d): Betrag und Phase der normierten $\tilde{v}$-Komponente 

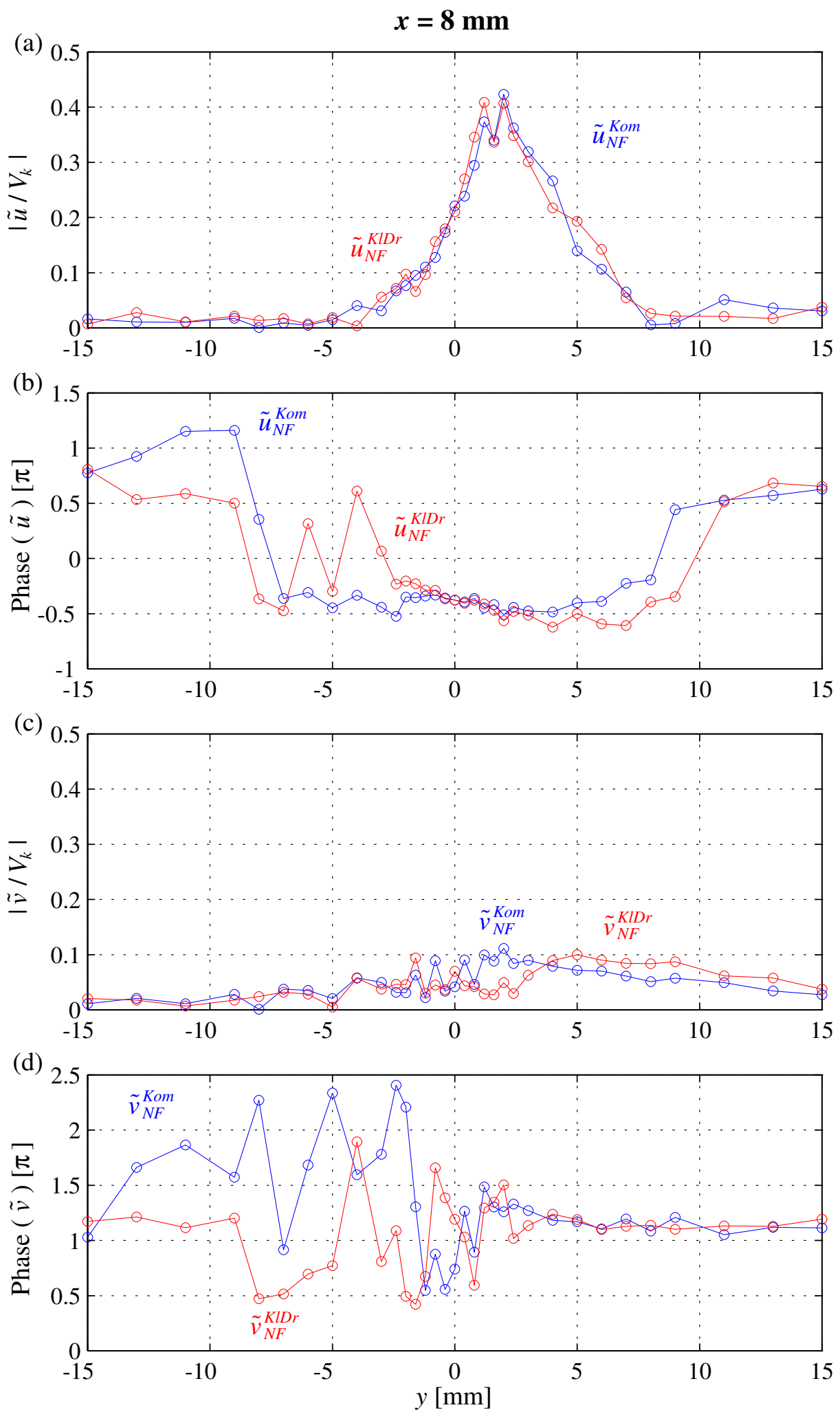

Abb. 5.11: Vergleich der Nahfelder im Kompensationsexperiment $\tilde{\psi}_{N F}^{\text {Kom }}$ (blaue Kurve) und bei Überlagerung von $\tilde{\psi}_{N F}^{K l}+\tilde{\psi}_{N F}^{D r}=\tilde{\psi}_{N F}^{K l D r}$ (rote Kurve) bei $x=8 \mathrm{~mm}$ für $1.6 \mathrm{~Hz}$ : (a) und (b): Betrag und Phase der normierten $\tilde{u}$-Komponente (c) und (d): Betrag und Phase der normierten $\tilde{v}$-Komponente 


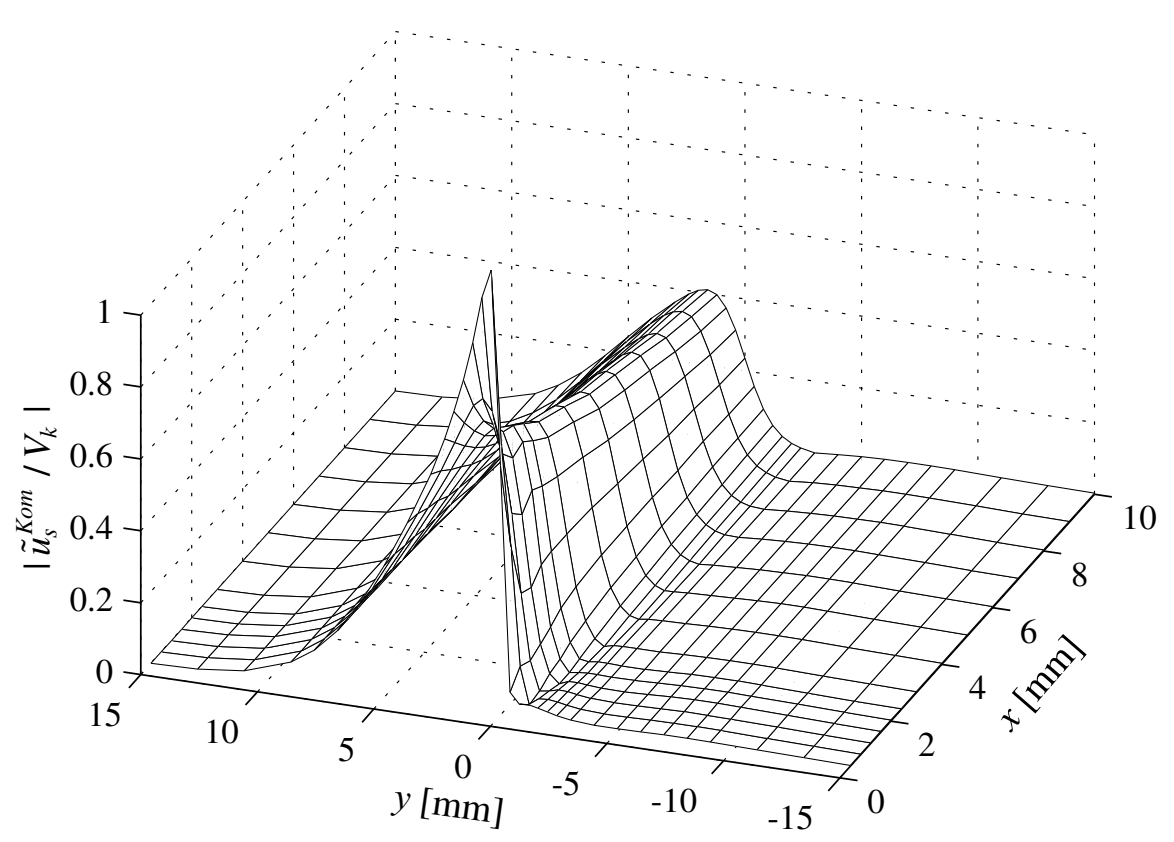

Abb. 5.12: Betrag der scheinbaren Geschwindigkeit $\tilde{u}_{s}^{K o m}$ im Kompensationsfeld bei $1.6 \mathrm{~Hz}$

Nahfeld im Kompensationsexperiment $\tilde{\psi}_{N F}^{\text {Kom }}$ lässt sich daher beschreiben durch:

$$
\tilde{\psi}_{N F}^{K o m}=\tilde{\psi}_{N F}^{K l}+M \cdot \tilde{\psi}_{N F}^{D r}
$$

\subsubsection{Vergleich des nichtanregenden mit dem anregenden Nahfeld}

Die wichtige Frage ist nun, woraus genau das Nahfeld besteht. Mit anderen Worten, wie man das Nahfeld beschreiben kann. Man kann zuerst vermuten, dass die $\tilde{u}$-Komponente des Nahfeldes aufgrund der $\partial \bar{u} / \partial y$-ähnlichen räumlichen Verteilung hauptsächlich durch die von dem anregenden Feld verursachte Verschiebung der Gleichströmung zustande gekommen ist. Der erste Versuch zur Beschreibung des Nahfeldes wäre somit ein Vergleich von $\tilde{u}_{N F}^{K o m}$ mit der scheinbaren Geschwindigkeit. Wenn $\bar{v} \approx 0$ und $\partial \bar{u} / \partial x \ll \partial \bar{u} / \partial y$, wird die scheinbare Geschwindigkeit im Kompensationsfeld $\tilde{u}_{s}^{K o m}$ bzw. $\tilde{v}_{s}^{\text {Kom }}$ gemäß Gleichung (4.11) beschrieben durch:

$$
\tilde{u}_{s}^{K o m}=-\tilde{\eta}_{A n F}^{K o m} \frac{\partial \bar{u}}{\partial y}, \quad \tilde{v}_{s}^{K o m} \approx 0 \quad \text { wobei } \quad \tilde{\eta}^{K o m}=\frac{\tilde{v}_{A n F}^{K o m}}{-i \beta}
$$

Abbildung 5.12 zeigt den Betrag von $\tilde{u}_{s}^{\text {Kom }}$ in einer dreidimensionalen Darstellung. Vergleicht man dieses Feld mit den in Abbildung 5.7(a) dargestellten $\tilde{u}_{N F}^{K o m}$-Feld, so ist zu erkennen, dass die beiden zur Anregung $(1.6 \mathrm{~Hz})$ kohärenten Wechselgeschwindigkeiten eine ähnliche Struktur haben. 

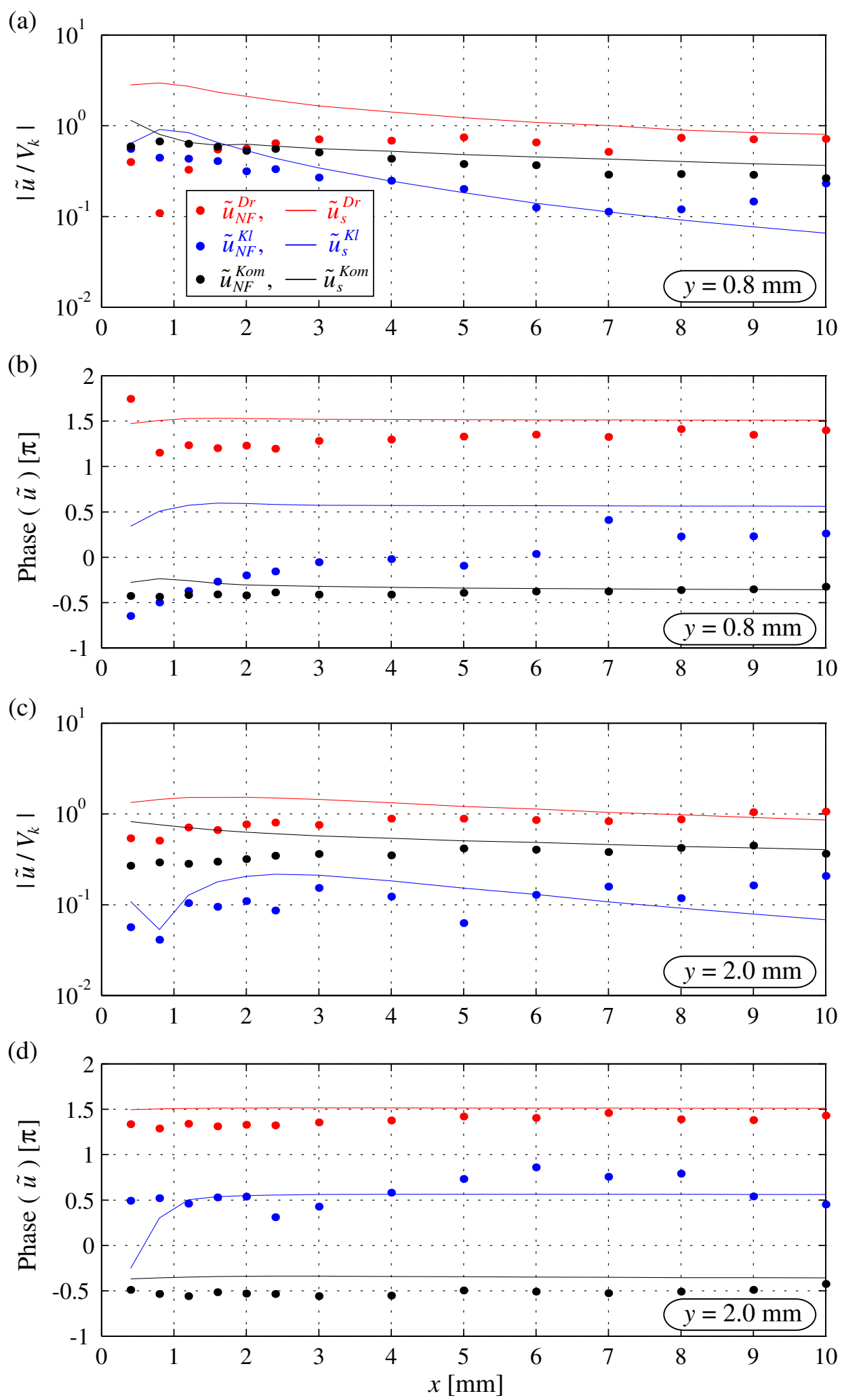

Abb. 5.13: Vergleich der $\tilde{u}$-Komponenten der Nahfelder $\tilde{u}_{N F}^{D r}$ (rote Punkte), $\tilde{u}_{N F}^{K l}$ (blaue Punkte) und $\tilde{u}_{N F}^{K o m}$ (schwarze Punkte) mit den scheinbaren Geschwindigkeiten $\tilde{u}_{s}^{D r}$ (rote Kurve), $\tilde{u}_{s}^{K l}$ (blaue Kurve) und $\tilde{u}_{s}^{K o m}$ (schwarze Kurve) in Abhängigkeit von der $x$ Koordinate für 1.6 Hz: (a) Betrag, (b) Phase bei $y=0.4 \mathrm{~mm}$, (c) Betrag und (d) Phase bei $y=2 \mathrm{~mm}$ 
Kapitel 5. Untersuchung der Nahfelder mittels Kompensationsexperiment

Da das Nahfeld $\tilde{\psi}_{N F}^{K o m}$ die angefachte Mode der KH-Wellen nicht anregt, wird es hier als ein „nichtanregendes Nahfeld" bezeichnet. $\tilde{\psi}_{N F}^{K l}$ bzw. $\tilde{\psi}_{N F}^{D r}$ sind dagegen anregende Nahfelder. Dies darf nicht verwechselt werden mit dem sogenannten anregenden Feld $\tilde{\psi}_{A n F}$, das das durch die Anregungsvorrichtungen erzeugte Potenzialfeld bei ruhendem Medium bezeichnet. Es besteht die Möglichkeit, dass man aus einem Vergleich zwischen den Nahfeldern eine entscheidende Größe bzw. Struktur, die zur Anregung der KHWellen führt, ermitteln kann. Da $\tilde{u}_{N F}^{D r}$ und $\tilde{u}_{N F}^{K o m}$, wie in Abbildung 4.24 (a) und 5.7 (a) zu sehen war, eine qualitative Ähnlichkeit mit dem $\partial \bar{u} / \partial y$-Profil haben, ist es sinnvoll, das nichtanregende und die anregenden Nahfelder bezüglich der scheinbaren Geschwindigkeit miteinander zu vergleichen.

Abbildung 5.13 zeigt die $\tilde{u}$-Komponente der drei verschiedenen Nahfelder $\tilde{u}_{N F}^{D r}$, $\tilde{u}_{N F}^{K l}$ und $\tilde{u}_{N F}^{K o m}$ (rote, blaue und schwarze Punkt) in Abhängigkeit von der $x$-Koordinate für $1.6 \mathrm{~Hz}$. Zum Vergleich sind dabei die scheinbaren Geschwindigkeiten $\tilde{u}_{s}^{D r}, \tilde{u}_{s}^{K l}$ und $\tilde{u}_{s}^{K o m}$ (rote, blaue und schwarze Kurve) aufgetragen. Da die Nahfelder praktisch nur im Hauptströmungsbereich in Erscheinung treten und man sich außerdem für den Bereich um die Hinterkante interessiert, wurde der Vergleich bei $y=0.8 \mathrm{~mm}$ und bei $y=2 \mathrm{~mm}$, jeweils für $0<x<10 \mathrm{~mm}$ durchgeführt.

Bei $y=2 \mathrm{~mm}$ ergeben sich gewisse Abweichungen zwischen $\tilde{u}_{N F}$ und $\tilde{u}_{s}$ in allen drei Feldern fast unabhängig von der $x$-Koordinate sowohl im Betrag als auch in der Phase. Trotz dieser Abweichungen kann man davon ausgehen, dass die beiden $\tilde{u}$-Komponenten in $x$-Richtung ähnlich verlaufen (siehe Abbildung 5.13 (c) und (d)). Bei $y=0.8 \mathrm{~mm}$ hängt dagegen die Abweichung zwischen $\tilde{u}_{N F}$ und $\tilde{u}_{s}$ von der $x$-Koordinate ab. $\tilde{u}_{N F}^{D r}$ und $\tilde{u}_{N F}^{K l}$ weichen für kleine $x$, also in Nähe der Hinterkante, deutlich von $\tilde{u}_{s}^{D r}$ bzw. $\tilde{u}_{s}^{K l} \mathrm{ab}$, während $\tilde{u}_{s}^{K o m}$ in diesem Bereich mit $\tilde{u}_{N F}^{K o m}$ sowohl im Betrag als auch in der Phase übereinstimmt. Für große $x$ dagegen (etwa $x>5 \mathrm{~mm}$ ) geben die Nahfelder die Verläufe der scheinbaren Geschwindigkeiten im wesentlichen quantitativ wieder (siehe Abbildung 5.13 (a) und b)). Aus dieser Auftragung gewinnt man außerdem den Eindruck, dass das nichtanregende Nahfeld im Gegensatz zu den anregenden Nahfeldern ziemlich vollständig durch die scheinbare Geschwindigkeit beschrieben werden kann.

Ob oder wie dieses Ergebnis zur Bestimmung einer maßgebenden Größe führen kann, die zur Anregung der KH-Wellen verantwortlich ist, ist allerdings noch nicht deutlich zu sehen. Da die scheinbare Geschwindigkeit, die an sich, wie in Kapitel 4 beschrieben wurde, nur durch eine scheinbare Verschiebung des Koordinatensystems entsteht, kann sie zumindest bei einer homogenen Verschiebung nicht zur Anregung von KH-Wellen führen. Es erscheint dann auch im inhomogenen Fall wahrscheinlich, dass sich KH-Wellen durch ein Nahfeld nicht anregen lassen, das sich als ein scheinbares Geschwindigkeitsfeld beschreiben lässt. Es wird dann verständlich, dass das nichtanregende Nahfeld mit der scheinbaren Geschwindigkeit besser übereinstimmt als die anregenden Nahfelder. Man sollte allerdings bedenken, dass $\left(\tilde{u}_{s}, \tilde{v}_{s}\right)$ wegen der Inhomogenität des Verschiebungsfeldes nicht divergenz frei ist (siehe Gleichung (4.13)). Die Verschiebungen der Gleichströmung induzieren somit durch die Divergenz von $\left(\tilde{u}_{s}, \tilde{v}_{s}\right)$ eine Ausgleichströmung, die ihrerseits zur Anregung der KH-Wellen führen kann.

Das würde bedeuten, dass die Amplitude der Instabilitätswelle mit dieser Ausgleichströmung im engen Zusammenhang steht. Die Divergenz $\tilde{T}$ des Feldes der scheinbaren 

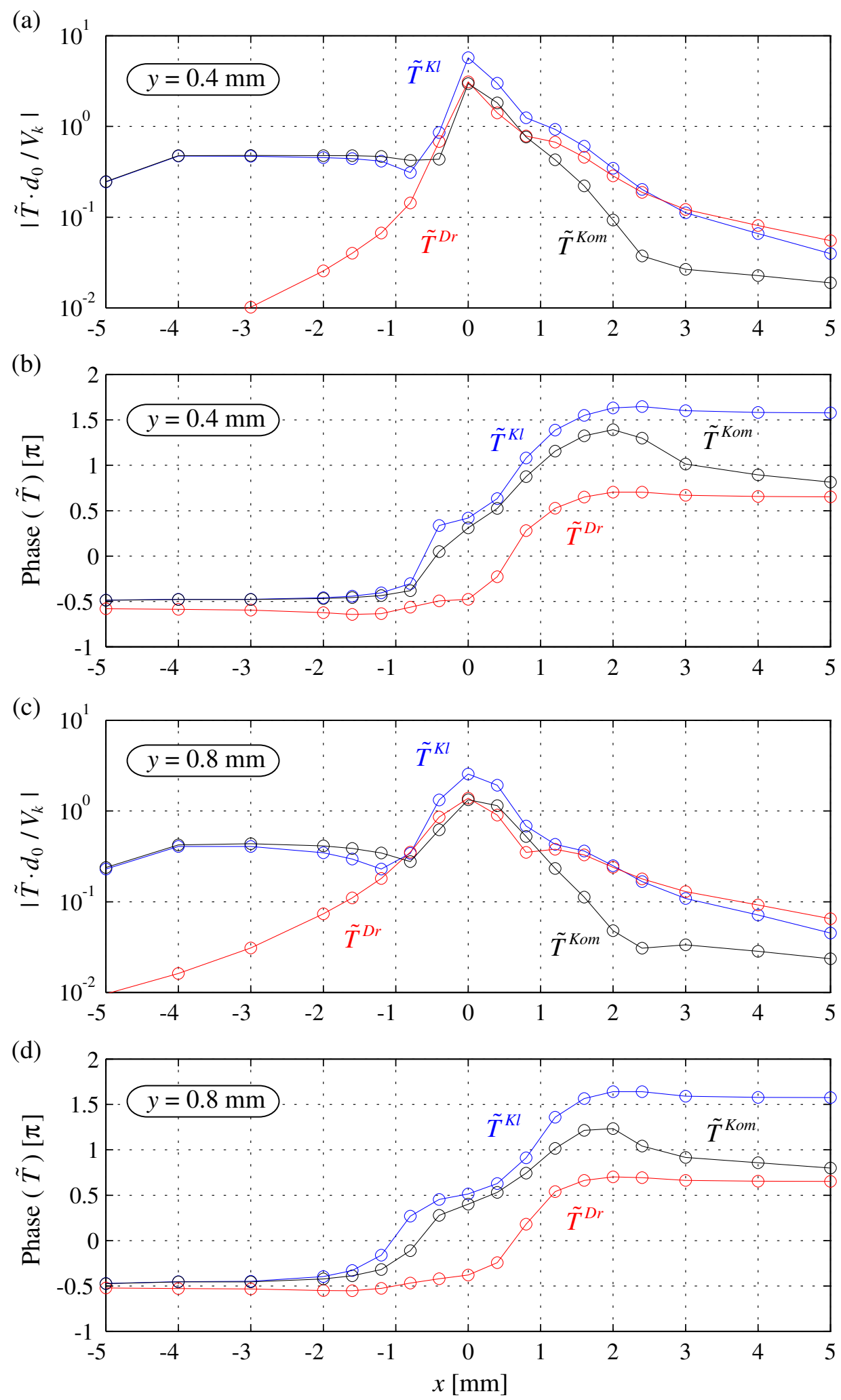

Abb. 5.14: Die mit $V_{k}$ und $d_{0}$ normierte Divergenz $\tilde{T}$ des scheinbaren Geschwindigkeitsfeldes in Abhängigkeit von der $x$-Koordinate für die drei verschiedenen Felder (Druckgradienten-Anregung $\tilde{T}^{D r}$ (rot), Schwingkanten-Anregung $\tilde{T}^{K l}$ (blau) und Kompensationsfeld $\tilde{T}^{\text {Kom }}$ (schwarz) bei $1.6 \mathrm{~Hz}$ 
Geschwindigkeit lässt sich gemäß Gleichung (4.13) schreiben als:

$$
\tilde{T}=\frac{1}{i \beta}\left(\frac{\partial \tilde{u}_{A n F}}{\partial x} \frac{\partial \bar{u}}{\partial x}+\frac{\partial \tilde{v}_{A n F}}{\partial x} \frac{\partial \bar{u}}{\partial y}+\frac{\partial \tilde{u}_{A n F}}{\partial y} \frac{\partial \bar{v}}{\partial x}+\frac{\partial \tilde{v}_{A n F}}{\partial y} \frac{\partial \bar{v}}{\partial y}\right)
$$

Da $\partial \bar{u} / \partial y$ im Hauptströmungsbereich etwa $0<x<10 \mathrm{~mm}$ um eine Größenordnung größer ist als $\partial \bar{u} / \partial x, \partial \bar{v} / \partial x$ und $\partial \bar{v} / \partial y$, liefert der zweite Term den Hauptbeitrag zu $\tilde{T}$ (für $y<0$ sind die Gradienten der Gleichströmung sehr klein).

Abbildung 5.14 zeigt die Divergenz der drei verschiedenen Felder $\tilde{T}^{D r}, \tilde{T}^{K l}$ und $\tilde{T}^{K o m}$ (rote, blaue und schwarze Kurve) in Abhängigkeit von der $x$-Koordinate bei $1.6 \mathrm{~Hz}$. $\tilde{T}$ ist dabei mit $V_{k}$ und $d_{0}$ dimensionlos gemacht. Die Phase ist jeweils auf die Geschwindigkeit der Anregungsvorrichtung und bei dem Kompensationsfeld auf die der Schwingkante bezogen. Da $\tilde{T}$, wie man aus Gleichung (5.8) erwartet, nur im Hauptströmungsbereich um die Hinterkante herum wesentlich von null abweicht und auch mit steigender $y$-Koordinate stark abfällt, wurde zum Vergleich der drei Felder der Bereich $-5 \mathrm{~mm}<x<5 \mathrm{~mm}$ bei $y=0.4 \mathrm{~mm}$ und bei $y=0.8 \mathrm{~mm}$ gewählt.

Es fällt auf, dass $\tilde{T}^{K l}$ und $\tilde{T}^{D r}$ im Bereich $x \gtrsim 2 \mathrm{~mm}$ bis auf die entgegengesetzte Phase einen sehr ähnlichen Verlauf zeigen. Da $\tilde{T}^{K o m}=\tilde{T}^{K l}+\tilde{T}^{D r}$, sollte dort $\tilde{T}^{K o m}$ also sehr klein sein. In der Tat fällt der Betrag von $\tilde{T}^{\text {Kom }}$ sowohl für $y=0.4 \mathrm{~mm}$ als auch für $y=0.8 \mathrm{~mm}$ stromab der Hinterkante mit steigender $x$-Koordinate zunächst wesentlich steiler ab als die beiden anderen. Stromauf der Hinterkante $(x<0)$ hat $\tilde{T}^{\text {Kom }}$ praktisch das gleiche Profil wie $\tilde{T}^{K l}$, da dort $\tilde{T}^{D r}$ sehr klein ist. Es ist daher zu vermuten, dass dieser Bereich für die Anregung der KH-Wellen nicht wichtig ist. Aus demselben Grund kann man davon ausgehen, dass der "Peak“, der etwa um $x=0$ liegt, erstaunlicherweise auch keinen Beitrag zur KH-Wellen-Anregung liefert. 


\section{Kapitel 6}

\section{Schlussfolgerung und Ausblick}

Das Strömungsfeld in der Nähe der Hinterkante einer überströmten Platte wurde experimentell untersucht, um Rückschlüsse auf den Anregungsmechanismus und die im Nahfeld für die Anregung der Kelvin-Helmholtz-Instabilität maßgebenden Größen zu gewinnen, die die Dynamik der ablösenden laminaren Scherschicht dominiert. Die Messungen wurden in einem Wasserkanal durchgeführt, der durch eine Splitterplatte in einen Haupt- und einen Nebenströmungsbereich unterteilt ist. Zwei Anregungsvorrichtungen, die die KH-Wellen künstlich anregen können, befinden sich in der Messstrecke des Kanals. Zum einen sind das zwei in die Seitenwände des Kanals integrierte Membranen, die einen normal zur Hinterkante gerichteten Druckgradienten erzeugen, und zum anderen eine schwingende Hinterkante. Die Geschwindigkeitsfelder der quasi-ebenen Strömung wurden mit Hilfe eines Laser-Doppler-Anemometers vermessen.

\subsection{Schlussfolgerung}

Die Untersuchung der Dynamik der von der Hinterkante abgelösten Scherschicht zeigt, dass das zur Anregung kohärente Wechselströmungsfeld nicht nur durch die konvektive Instabilität dominiert wird, sondern auch mit der instationären Ablösebedingung im engen Zusammenhang steht. Das Wechselströmungsfeld wird zur Untersuchung der Wechselwirkung zwischen der Gleichströmung und dem anregenden Feld in drei Teile zerlegt, nämlich in das anregende Feld $\tilde{\psi}_{A n F}$ selbst, das Feld der KH-Wellen $\tilde{\psi}_{K H}$ und das Nahfeld $\tilde{\psi}_{N F}$, das nicht zu den zwei ersten Feldern gehört. Die anregenden Felder, das sind die Felder, die bei ruhendem Medium durch die beiden Anregungsvorrichtungen erzeugt werden, wurden in der vorliegenden Arbeit durch eine numerische Simulation mittels der "CIP-Methode" bestimmt (siehe Anhang A). Bei der Beschreibung des Feldes der KH-Wellen $\tilde{\psi}_{K H}$ gewinnt man die folgenden Erkenntnisse:

- In dem Bereich, in dem $(\partial \bar{u} / \partial x) / \beta \ll 1$ gilt, wird $\tilde{\psi}_{K H}$ durch das gemäß RayleighGleichung bestimmte theoretische KH-Wellenprofil sehr gut beschrieben.

- Der Ansatz mit den lokalen Wellenzahlen $\left(\alpha, \alpha^{*}\right)$ und den Eigenfunktionen $\left(\psi_{0}\right.$, $\left.\psi_{0}^{*}\right)$ sowie den Amplituden $\left(C_{A}, C_{D}\right)$ kann verwendet werden, um die Inhomogenität des $\bar{u}$-Profils zur Beschreibung von $\tilde{\psi}_{K H}$ näherungsweise zu berücksichtigen. 
- Die lokale Amplitude $C_{A}$ der angefachten KH-Welle, die durch Anpassung an Messwerte bestimmt wird, ist ein theoretisch sinnvoller Parameter, da sie gut mit der WKB-Näherung beschrieben werden kann.

- Die $x$-Abhängigkeit der Amplitude $C_{D}$ der gedämpften KH-Welle zeigt, dass aufgrund der Inhomogenität des $\bar{u}$-Profils eine Wechselwirkung zwischen den beiden KH-Moden stattfindet: die angefachte wird in die gedämpfte Mode gestreut.

- Werden die gemessenen Wechselfelder bei allen Frequenzen (0.8, 1.2, 1.6, 2.0 und $2.4 \mathrm{~Hz}$ ) mit gleicher Geschwindigkeit der Membranen der DruckgradientenAnregung erzeugt, so zeigt sich, dass $C_{A}$ systematisch mit abnehmender Frequenz stark zunimmt. Dies bleibt auch so - etwas schwächer ausgeprägt -, wenn man anstelle der Geschwindigkeit die Auslenkung des anregenden Feldes als Funktion der Frequenz konstant lässt. Man findet also an dieser Stelle keine einfache Beziehung zwischen den Anregungsparametern und der resultierenden KH-WellenAmplitude.

Das Nahfeld kann nun aus dem gemessenen Gesamtfeld ermittelt werden. Dieses Ergebnis führt zu einer Tatsache, dass die Wechselwirkung zwischen der Gleichströmung und dem anregenden Feld nicht nur durch $\tilde{\psi}_{K H}$ sondern auch durch $\tilde{\psi}_{N F}$ beschrieben wird. Da die Form von $\tilde{\psi}_{N F}$ etwa proportional zu dem $\partial \bar{u} / \partial y$-Profil ist, wurde die Hypothese untersucht und bestätigt, dass ein beträchtlicher Teil des Nahfeldes durch die von dem anregenden Feld verursachte Verschiebung der Scherschicht entstanden gedacht werden kann. Diese Verschiebung, die ein Fluidteilchen ohne überlagerte Gleichströmung erfahren würde, ist rein fiktiv und hat nur wenig mit einer tatsächlichen Verschiebung zu tun.

Zur weiteren Untersuchung des Nahfeldes wurden Kompensationsexperimente durchgeführt, in denen die durch die zwei Anregungsvorrichtungen erzeugten Wechselfelder so überlagert werden, dass die angefachte Mode der KH-Wellen im Fernfeld verschwindet.

Das beim Kompensationsexperiment übrig bleibene Nahfeld $\tilde{\psi}_{N F}^{K o m}$, also das „nichtanregende Nahfeld", gehorcht der Beziehung $\tilde{\psi}_{N F}^{K o m}=\tilde{\psi}_{N F}^{K l}+M \cdot \tilde{\psi}_{N F}^{D r}$, ist also keinen nichtlinearen Effekten unterworfen.

Ein Vergleich des nichtanregenden Nahfeldes $\tilde{\psi}_{N F}^{K o m}$ mit den anregenden Nahfeldern $\tilde{\psi}_{N F}^{K l}$ bzw. $\tilde{\psi}_{N F}^{D r}$ führt zu dem wichtigsten Ergebnis der Untersuchung: Das nichtanregende Nahfeld ist durch das scheinbare Geschwindigkeitsfeld $\left(\tilde{u}_{s}, \tilde{v}_{s}\right)$ vollständiger beschrieben als die anregenden Nahfelder. Dieses Ergebnis ist sehr interessant, da die scheinbare Geschwindigkeit durch eine scheinbare Verschiebung des Koordinatensystems entsteht, die im homogenen Fall nicht zur Anregung der KH-Wellen führen kann. Es ist dann zu vermuten, dass sich KH-Wellen im inhomogenen Fall auch nicht durch ein Nahfeld anregen lassen, das sich als ein scheinbares Geschwindigkeitsfeld darstellen lässt. Bei einer inhomogenen Verschiebung befinden sich allerdings Quellen in dem scheinbaren Feld, die zur Anregung der KH-Wellen führen können, da die Divergenz dieses Feldes nicht verschwindet. Der räumliche Verlauf der Divergenz von $\left(\tilde{u}_{s}, \tilde{v}_{s}\right)$ zeigt, dass er bei dem nichtanregenden Nahfeld stromab der Hinterkante mit steigender $x$-Koordinate deutlich steiler abfällt als bei den anregenden Nahfeldern und danach etwa konstant 
bleibt. Es wäre demnach möglich, dass man in dem Bereich, in dem die Divergenz des scheinbaren Geschwindigkeitsfeldes bei dem nichtanregenden Nahfeld wesentlich kleiner als bei den anregenden Nahfeldern ist, einen Keim finden kann, der für die Anregung der Kelvin-Helmholtz-Instabilitätswellen verantwortlich ist.

Das Kompensationsexperiment unterstützt nebenbei die Vermutung der Wechselwirkung zwischen der angefachten und der gedämpften Mode von KH-Wellen, da $C_{D}^{K o m}$ wegen der nichtvorhandenen angefachten Mode nicht mit steigender $x$-Koordinate zunimmt, sondern konstant wird.

\subsection{Ausblick}

Die durchgeführten Untersuchungen geben einen Hinweis darauf, dass die von einem inhomogenen anregenden Feld verursachte scheinbare Verschiebung im Nahfeld mit einer Anregungsgröße der KH-Instabilität in einem engen Zusammenhang steht. Zur Bestimmung einer solchen Größe ist eine weitere Untersuchung der Divergenz des scheinbaren Geschwindigkeitsfeldes nützlich. Ein interessanter Versuch wäre die Bestimmung eines Potenzialströmungsfeldes, das von der Divergenz des scheinbaren Geschwindigkeitsfeldes erzeugt wird, da die Divergenz von $\left(\tilde{u}_{s}, \tilde{v}_{s}\right)$ als eine Quellen-Senken-Verteilung angesehen werden kann. Dieses so bestimmte Potenzialfeld könnte in der Lage sein zur Anregung der KH-Wellen zu führen und dessen räumliche Verteilung, also z. B. das Stromlinienbild, sollte zwischen dem anregenden und dem nichtanregenden Nahfeld unterschiedlich sein. Man kann allerdings an dieser Stelle noch nicht das zur Anregung der KH-Wellen maßgebende Strömungsfeld dahingehend einschränken, dass es wirbelfrei sein soll. Es ist nur zu vermuten, dass die Wirbeligkeitsverteilung im Nahfeld nicht wichtig ist, da die bisherigen Ergebnisse zeigen, dass das wirbelfreie Störungsfeld eine wesentliche Bedeutung für die Anregung der KH-Wellen hat (DIERKEN [8] und REBEL [20]).

Das Nahfeld ließe sich somit weiter zerlegen, wenn man die durch die Divergenz von $\left(\tilde{u}_{s}\right.$, $\tilde{v}_{s}$ ) gewonnene Ausgleichströmung von dem hier bestimmten Nahfeld abzieht. Wenn der weiter verbleibende Rest keinen Unterschied zwischen dem anregenden und dem nichtanregenden Nahfeld zeigen würde, würde das bedeuten, dass das durch die Divergenz der scheinbaren Geschwindigkeit induzierte Strömungsfeld tatsächlich für die Anregung der KH-Wellen verantwortlich ist.

Es stellt sich als weitere Frage, welches Feld, $\tilde{\psi}_{A l l}^{K o m}$ oder $\tilde{\psi}_{A n F}^{K o m}$ im Nahfeld der Kante besser geeignet ist, in dem Feld nach der Anregungsgröße der KH-Instabilität zu suchen. DiERKEN [8] untersuchte das Feld $\tilde{\psi}_{\text {All }}^{\text {Kom }}$, wobei das Kompensationsexperiment mittels der Zylinder- und der Druckgradienten-Anregung durchgeführt worden ist. Dabei zeigte sich, dass $\partial \tilde{v}_{A l l}^{K o m} / \partial x$ außerhalb der Stokesschicht verschwindet, während $\tilde{v}_{A n F}^{K o m}$ mit steigender $x$-Koordinate in diesem Bereich monoton abnimmt. In dem durch die Schwingkanten-Anregung kompensierten Feld verschwindet $\partial \tilde{v}_{A l l}^{\text {Kom }} / \partial x$ im Bereich außerhalb der Stokesschicht dagegen nicht, so dass die von DiERKEN gemachte Beobachtung somit nicht verallgemeinert werden kann. Damit stellt sich die Frage, ob denn in dem von DiERKEN untersuchten Fall die Divergenz $\tilde{T}$ ebenfalls klein wird im Vergleich zu dem primären Feldern, die zur Anregung von KH-Wellen führen. 
Darüber hinaus wären weitere Anregungsvorrichtungen hilfreich, um die bisherigen Beobachtungen verallgemeinern bzw. weiter spezifizieren zu können.

Für die theoretische Beschreibung des KH-Wellen-Feldes in der inhomogenen Gleichströmung hinter der Ablösekante hat sich die WKB-Methode als geeignet erwiesen. Es wäre nun interessant, dass ein Ansatz bei der WKB-Methode verwendet wird, in dem nicht nur die angefachte, sondern auch die gedämpfte Mode der KH-Wellen gleichzeitig berücksichtigt werden. Man kann dann erwarten, dass die Wechselwirkung zwischen den beiden Moden theoretisch beschrieben wird und sie mit den hier bestimmten lokalen Amplituden $C_{A}$ und $C_{D}$ verglichen wird.

Aufgrund der Komplexität des Untersuchungsgegenstandes und der Grenzen der Messgenauigkeit sowie der räumlichen Auflösung werden numerische Simulationen für die Untersuchung der instationären Ablösung durchgeführt (z.B. BeBBer [1] und Schmitz [23]). Die Fortsetzung der Untersuchungen in simulierten Strömungen ist wahrscheinlich weniger aufwändig als der Aufbau neuer realer Experimente. 


\section{Anhang A}

\section{Das anregende Strömungsfeld}

Die gemessene Wechselgeschwindigkeit bei Überlagerung einer Gleichströmung besteht sowohl aus dem anregenden Feld als auch aus einem Feld, das durch die Wechselwirkung zwischen dem Feld der Gleichströmung und dem anregenden Feld erzeugt wird. Um diese Wechselwirkung zu untersuchen, soll das anregende Feld möglichst genau bestimmt werden, so dass man es aus der gemessenen Wechselgeschwindigkeit subtrahieren kann.

In diesem Anhang A wird daher die Strömung untersucht, die bei im Mittel ruhendem Medium durch die sinusförmig schwingende Hinterkante bzw. durch die harmonische Druckgradienten-Anregung entsteht. Es wurde durch die bisherigen Arbeiten gezeigt, dass das anregende Feld der Druckgradienten-Anregung durch eine Potenzialtheorie unter Berücksichtung der Kanalwände gut beschrieben wird (DIERKEN [8], GRAF [13]). Es ist allerdings nicht zu erwarten, dass die akustische Grenzschicht mit der Potenzialtheorie dargestellt wird. In dieser Arbeit wird daher für die theoretische Beschreibung eine numerische Simulation durchgeführt und die Ergebnisse werden mit experimentellen Daten verglichen.

\section{A.1 Das numerische Verfahren}

Da die vorliegende Arbeit nicht um eine numerische Untersuchung geht, soll hier nur der Überblick der verwendeten Methode kurz vorgestellt werden. Die Gültigkeit der numerischen Lösung wird dabei nur durch den Vergleich mit den Messergebnissen überprüft.

\section{A.1.1 Die CIP-Methode}

Die zweidimensionale, zeitabhängige, inkompressible Navier-Stokes Gleichung

$$
\frac{\partial \vec{u}}{\partial t}+(\vec{u} \cdot \vec{\nabla}) \vec{u}=-\vec{\nabla} p+\frac{1}{R e_{f}} \Delta \vec{u}
$$

sowie die Kontinuitätsgleichung

$$
\vec{\nabla} \cdot \vec{u}=0
$$


werden mittels einer „Cubic Interpolated Propagation (CIP)-Methode“(YABE \& AOKI [26]) für zwei parallel angeordnete Wände mit einer dazwischen liegenden Splitterplatte, deren Hinterkante im Rechengebiet liegt, numerisch gelöst. Dabei ist $\vec{u}=(u, v)^{T}$ die Geschwindigkeit (hier in kartesischen Koordinaten angegeben) und $p$ der kinematische Druck bezogen auf die Dichte des Fluids $\varrho$. Alle Größen sind mit einer charakteristischen Länge $L$ (hier: halber Abstand der Kanalwände $h$ ) und einer charakteristischen Frequenz $f$ (entspricht hier der Anregungsfrequenz der Membranen bzw. der Schwingkante) dimensionslos gemacht. Damit ist $1 / \operatorname{Re}_{f}$ die dimensionslose Viskosität.

Die Idee der CIP-Methode ist es, zuerst die Navier-Stokes Gleichung (A.1) in den Konvektionsterm und den Nichtkonvektionsterm zu zerlegen und danach den Konvektionsterm durch eine Polynominterpolation numerisch zu lösen. Der Nichtkonvektionsterm kann zum Beispiel mittels eines Finite-Differenzen-Verfahrens gelöst werden. Eine zweidimeisionale Konvektionsgleichung, die in der folgenden Form dargestellt wird,

$$
\frac{\partial f}{\partial t}+U_{c} \frac{\partial f}{\partial x}+V_{c} \frac{\partial f}{\partial y}=0
$$

hat eine analytische Lösung:

$$
f(x, y, t)=f\left(x-U_{c} t, y-V_{c} t, 0\right)
$$

wobei $U_{c}$ bzw. $V_{c}$ positive Konstanten sind. Die Gleichung (A.3) bedeutet, dass die Lösung bei $t>0$ durch die Verschiebung (in $x$-Richtung um $U_{c} t$, in $y$-Richtung um $\left.V_{c} t\right)$ der Lösung bei $t=0$ bestimmt wird. Es ist daher wichtig, dass sich die Lösung während der numerischen Rechnung möglichst wenig verändert. Es gibt verschiedene Möglichkeiten, die Gleichung (A.3) numerisch zu lösen (z B. Upwind-Methode oder Lax-Wendroff-Methode).

Bei der CIP-Methode erhält man zur numerischen Lösung der Gleichung (A.3) ein Polynom 3. Grades mit 10 freien Parametern in der folgenden Form, mit dem die Funktion $f(x, y)$ interpoliert wird:

$$
\begin{aligned}
f^{n}(x, y)=C_{30} x^{3}+ & C_{21} x^{2} y+C_{12} x y^{2}+C_{03} y^{3}+C_{20} x^{2}+ \\
& +C_{11} x y+C_{02} y^{2}+C_{10} x+C_{01} y+C_{00}
\end{aligned}
$$

dabei ist $n$ der Zeitindex. Um diese 10 freien Parameter zu bestimmen, benutzt man neben den Werten $\left(f_{0,0}^{n}, f_{1,0}^{n}, f_{0,1}^{n}, f_{1,1}^{n}\right)$ die ersten Ableitungen der nach $x$ und $y:\left(\partial f_{0,0}^{n} / \partial x\right.$, $\partial f_{0,0}^{n} / \partial y, \partial f_{1,0}^{n} / \partial x, \partial f_{1,0}^{n} / \partial y, \partial f_{0,1}^{n} / \partial x, \partial f_{0,1}^{n} / \partial y$ ) (siehe Abbildung A.1). Die zeitliche Entwicklung gemäß Gleichung (A.4) wird in der folgenden Form durchgeführt.

$$
f^{n+1}\left(x_{0}, y_{0}\right)=f^{n}\left(x_{0}-U_{c} \Delta t, y_{0}-V_{c} \Delta t\right)
$$

Es hat sich herausgestellt, dass sich die Form der Funktion $f(x, y)$, die mittels dieser Polynom-Interpolation durch die Gleichung (A.5) numerisch berechnet wird, mit fortschreitender Zeit sehr stabil verhält, besonders wenn die Funktion $f(x, y)$ unstetig ist (Yabe, Uchiumi \& Ogata [27]). Es gibt einen weiteren Vorteil bei der CIP-Methode: Da das Profil $f(x, y)$ mit der differenzierbaren Funktion (Gleichung (A.5)) interpoliert 


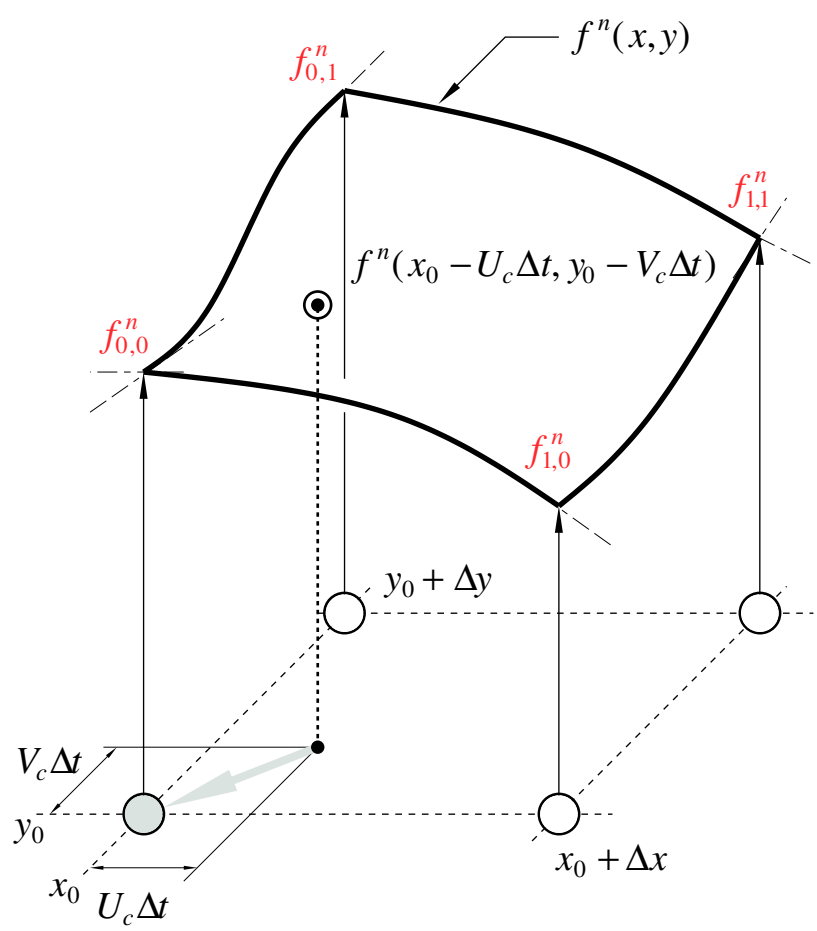

Abb. A.1: Die numerische Rechnung bei dem Konvektionsterm mittels der CIPMethode

wird, ist es nicht nötig, $f^{n}(x, y)$ weiter numerisch zu differenzieren, um die Ableitung von $f^{n}(x, y)$ zu berechnen, sondern man kann direkt von Gleichung (A.5) die räumliche Ableitung bestimmen.

$$
\begin{gathered}
\left\{\begin{array}{l}
\partial f^{n}(x, y) / \partial x=3 C_{30} x^{2}+2 C_{21} x y+C_{12} y^{2}+2 C_{20} x+C_{11} y+C_{10} \\
\partial f^{n}(x, y) / \partial y=3 C_{03} y^{2}+2 C_{12} x y+C_{21} x^{2}+2 C_{02} y+C_{11} x+C_{01}
\end{array}\right. \\
\left\{\begin{array}{l}
\partial f^{n+1}\left(x_{0}, y_{0}\right) / \partial x=\partial f^{n}\left(x_{0}-U_{c} \Delta t, y_{0}-V_{c} \Delta t\right) / \partial x \\
\partial f^{n+1}\left(x_{0}, y_{0}\right) / \partial y=\partial f^{n}\left(x_{0}-U_{c} \Delta t, y_{0}-V_{c} \Delta t\right) / \partial y
\end{array}\right.
\end{gathered}
$$

\section{A.1.2 Das verwendete räumliche Gitter}

Zur numerischen Lösung der Navier-Stokes Gleichung sowie der Kontinuitätsgleichung müssen neben der Zeit auch zwei Raumrichtungen diskretisiert werden. Abbildung A.2 zeigt ein zweidimensionales versetztes Gitter mit zwei Wänden und einer Splitterplatte, das bei der numerischen Rechnung verwendet wird.

Der Vorteil dieser versetzten Anordnung der Gitterpunkte gegenüber einer Anordnung, bei der der Druck $P$ und die zwei Komponenten der Geschwindigkeit $u$ bzw. $v$ am gleichen Ort abgetastet werden, liegt darin, dass es keine unerwünschten Druckoszillationen gibt. Ein weiterer Vorteil ist, dass bei der zu lösenden Poissongleichung, die aus der Gleichung (A.1) sowie Gleichung (A.2) folgt: 


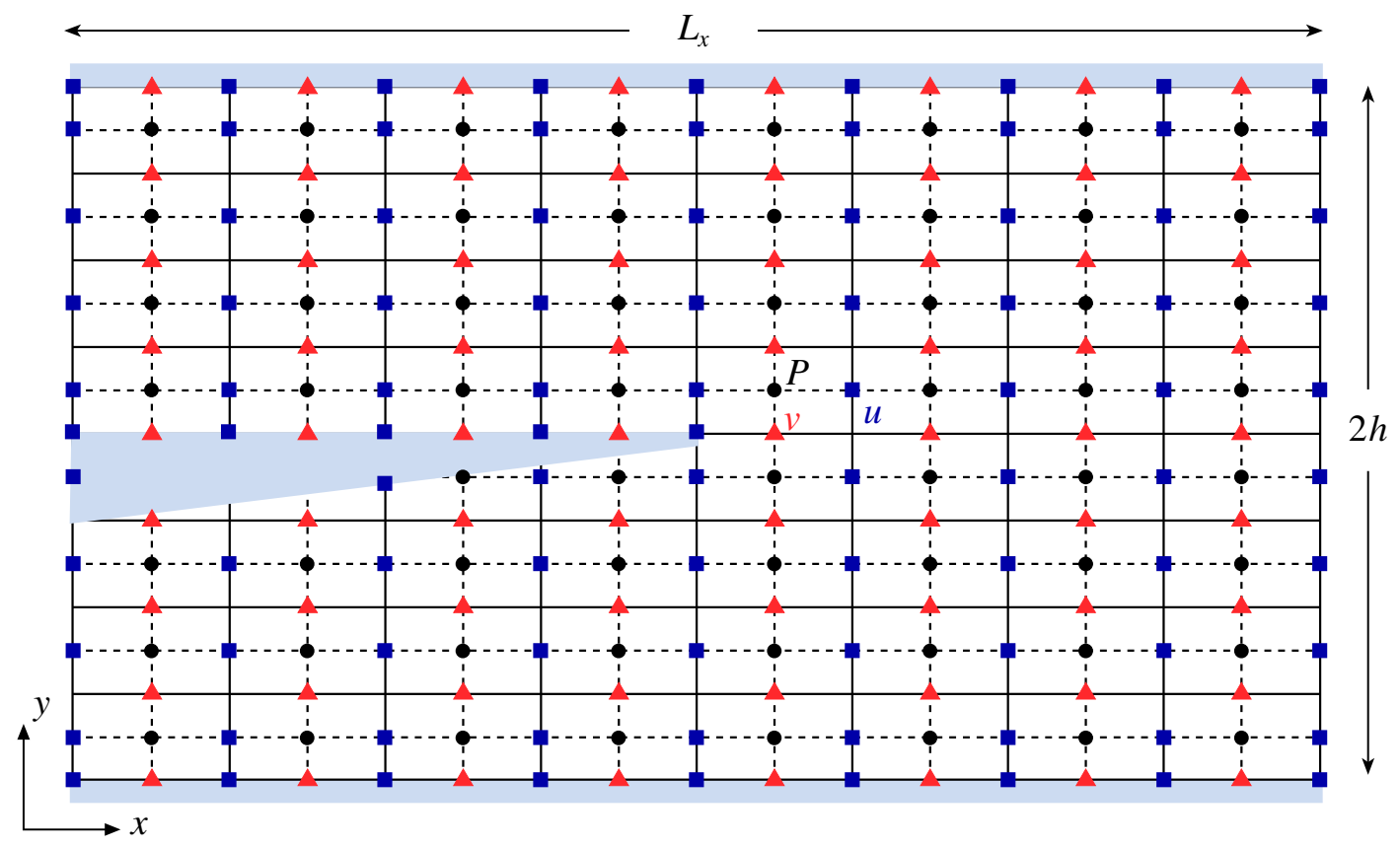

Abb. A.2: Das bei der numerischen Rechnung verwendete Gitter
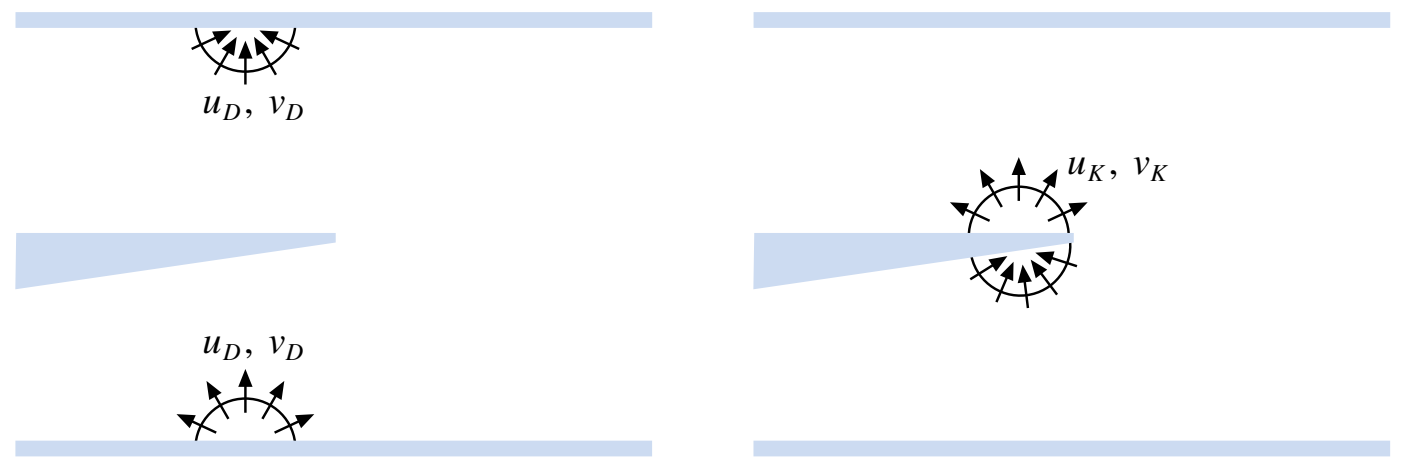

Abb. A.3: Das Modell der Anregungsvorrichtungen bei der numerischen Rechnung durch eine Verteilung von Quellen und Senken. Die linke Darstellung zeigt die Druckgradient-Anregung, die rechte Seite ist die Schwingkanten-Anregung. 


$$
\Delta P=-\operatorname{div}((\vec{u} \cdot \nabla) \vec{u})+\frac{1}{R e_{f}} \operatorname{div} \cdot \Delta \vec{u},
$$

keine Randwerte für den Druck $P$ benötigt werden. An den Wänden bzw. der Splitterplatte werden die sogenannten „Ghost-Punkten“, die in der Regel zur Implementierung der Randbedingungen eingeführt werden, verwendet, da man die Geschwindigkeit direkt an der Wand vorgeben möchte.

\section{A.1.3 Randbedingungen}

Um die Navier-Stokes Gleichung numerisch lösen zu können, benötigt man Randbedingungen. An der rechten und der linken Seite des Kanals (siehe Abbildung A.2) werden bei der $u$-Komponente Neumann-Randbedingungen vorgeschrieben:

$$
\frac{\partial u(0, y, t)}{\partial x}=0, \quad \frac{\partial u\left(L_{x}, y, t\right)}{\partial x}=0
$$

Die Gleichung (A.10) zusammen mit der Kontinuitätsgleichung bedeutet, dass die $v$ Komponente an diesen beiden Rändern null sein muss:

$$
v(0, y, t)=0, \quad v\left(L_{x}, y, t\right)=0
$$

Es ist daher wichtig, dass man eine genügende Kanallänge $L_{x}$ hat, damit die $v$ Komponente an beiden Seiten nicht zu null gezwungen werden muss.

An den Kanalwänden sowie an der Splitterplatte gilt die Haftbedingung unter Berücksichtigung der Wandbewegung entsprechend einer Druckgradienten-Anregung und der Bewegung der Hinterkante für die Schwingkanten-Anregung. Allerdings ist dabei angenommen, dass sich die Wände sowie die Hinterkante nicht wirklich im verwendeten Gitter bewegen, sondern die Bewegungen durch eine Verteilung der Quellen bzw. der Senken genähert werden (siehe Abbildung A.3).

$u_{D}(x, y, t), \quad v_{D}(x, y, t)$ ist dabei die Quellen- und Senkenverteilung für die Druckgradienten-Anregung, $\quad u_{K}(x, y, t), \quad v_{K}(x, y, t)$ die Verteilung für die Schwingkanten-Anregung.

\section{A.1.4 Das Flussdiagramm der numerischen Rechnung}

Abbildung A.4 zeigt ein Flussdiagramm zur numerischen Lösung der Gleichung (A.1) mittels der CIP-Methode. Nachdem man die Anfangsbedingung und Randbedingungen vorgegeben hat, erhält man die Poissongleichung für den Druck (Gleichung (A.9)). Um diese Poissongleichung numerisch zu lösen, wird eine übliche Lösungsmethode, das sogenannte „Successive Over Relaxation“(SOR) -Verfahren, verwendet. Die Iteration dauert dabei solange, bis die Differenz zwischen der rechten Seite und der linken Seite von Gleichung (A.11) kleiner als $10^{-8}$ wird. Dann wird der Nichtkonvektionsterm der Geschwindigkeit mit einem sogenannten „Forward in Time and Centered Difference in Space"(FTCS)-Schema berechnet. Anschließend wird der Konvektionsterm mit der oben vorgestellten CIP-Methode ausgerechnet. 


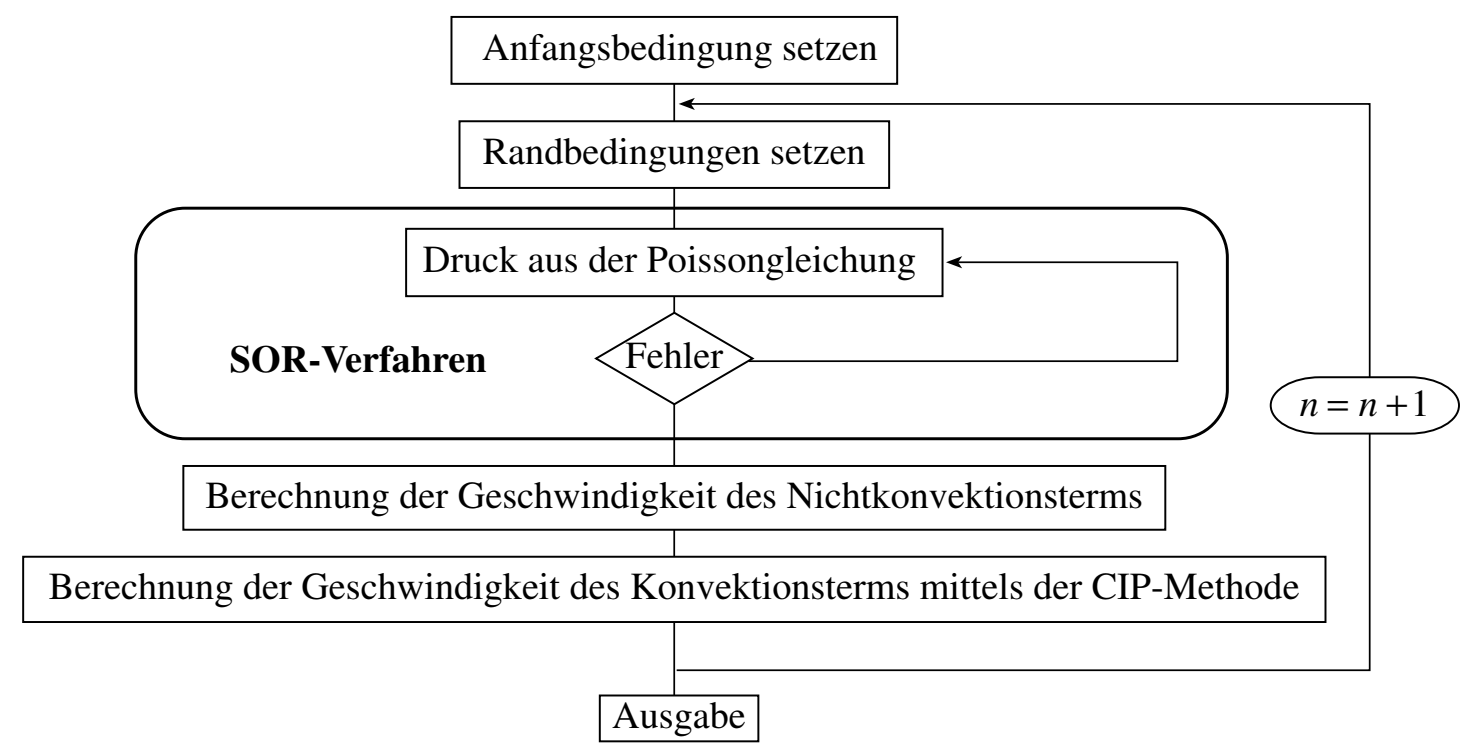

Abb. A.4: Das Flussdiagram der numerischen Rechnung mittels der CIP-Methode

\section{A.2 Vergleich der numerischen Lösung mit Mess- werten}

In diesem Abschnitt werden zuerst Ergebnisse der numerischen Lösung dargestellt und danach mit Messungen bei ruhendem Medium verglichen. Abbildung A.5 und A.6 zeigen numerisch berechnete Übersichtsdarstellungen der $\tilde{u}$ - und $\tilde{v}$-Komponente des durch den Druckgradienten bzw. durch die Schwingkante angeregten Feldes, die Anregungsfrequenz ist dabei $2 \mathrm{~Hz}$.

Da bei der Druckgradienten-Anregung im Bereich der Splitterplatte die Dicke der Stokesschicht $\delta_{S t}$ die einzige charakteristische Längenskala der Strömung ist, normiert man die Geschwindigkeit mit dem $\tilde{v}$-Wert $V_{S t}$ am Punkt $\left(x=\delta_{S t}, y=0\right)$. Bei der Schwingkanten-Anregung wird dagegen die Geschwindigkeit der Hinterkante $V_{k}=\left|-\beta K_{A}\right|$ als eine Normierungsgröße verwendet, wobei $\beta$ die Kreisfrequenz und $K_{A}$ die Amplitude der Hinterkante ist (siehe Abbildung A.7).

Das durch den Druckgradienten angeregte Feld ohne Gleichströmungsüberlagerung entspricht einer Umströmung der Hinterkante der Splitterplatte, deren Richtung mit der Periode der Membranen wechselt. Man erkennt daher in Abbildung A.5, dass sich die Struktur der $\tilde{u}$-Komponente im Bereich $x<0$ konzentriert, während die $\tilde{v}$-Komponente wesentlich im Bereich $x>0$ vorhanden ist. Man sieht, dass das Geschwindigkeitsfeld aufgrund des Öffnungswinkels der Plattenhinterkante asymmetrisch ist.

Bei der Schwingkanten-Anregung konzentriert sich die Wechselströrung um die Hinterkante. Das Konturlinienbild zeigt, dass die Geschwindigkeit mit steigender $x$ Koordinate steiler abfällt als bei der Druckgradienten-Anregung. 

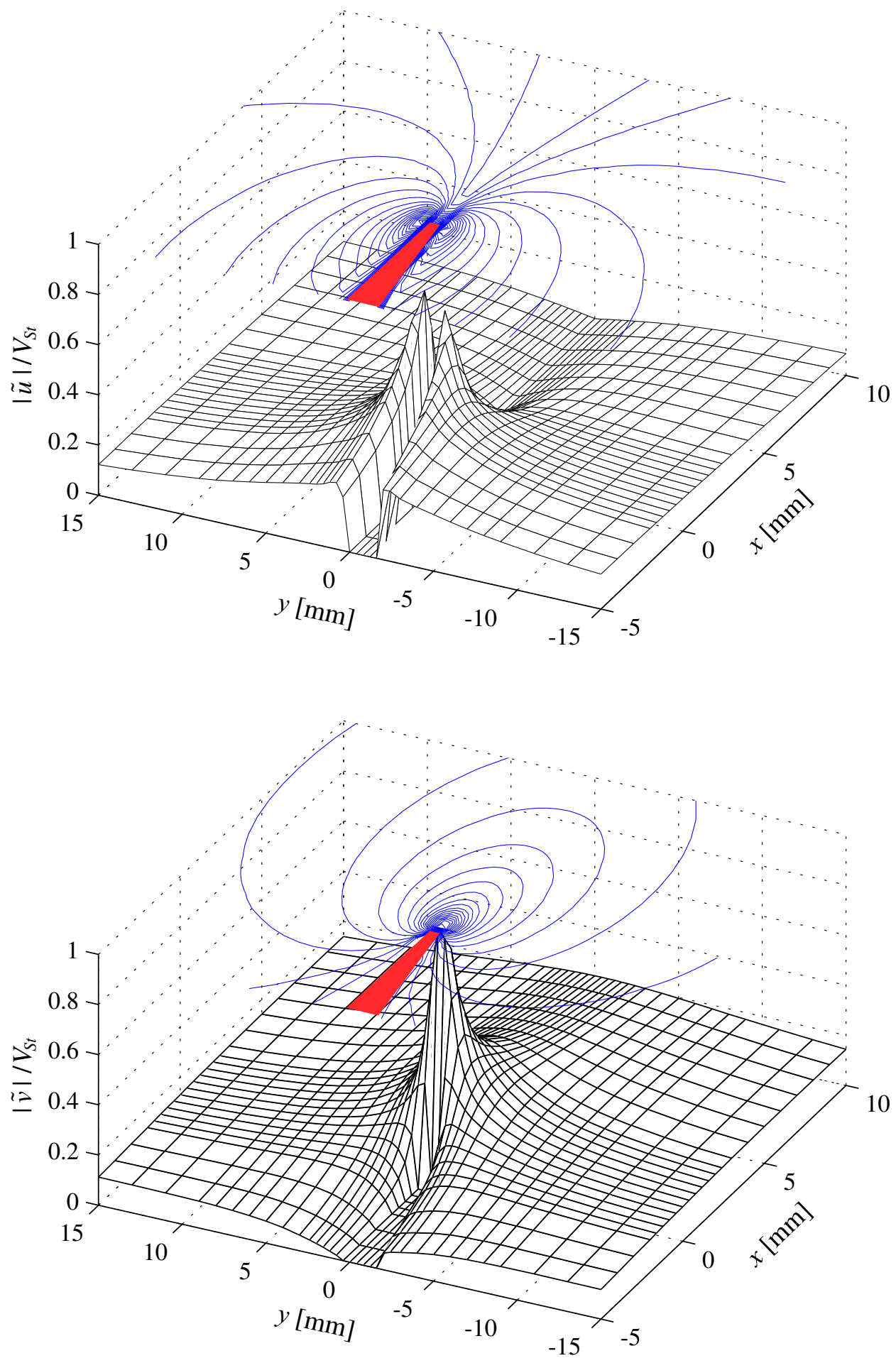

Abb. A.5: Amplitude der Wechselgeschwindigkeit im Bereich der Plattenhinterkante bei Druckgradienten-Anregung mit $2 \mathrm{~Hz}$ : $\tilde{u}$-Komponente (oben) und $\tilde{v}$-Komponente (unten) 

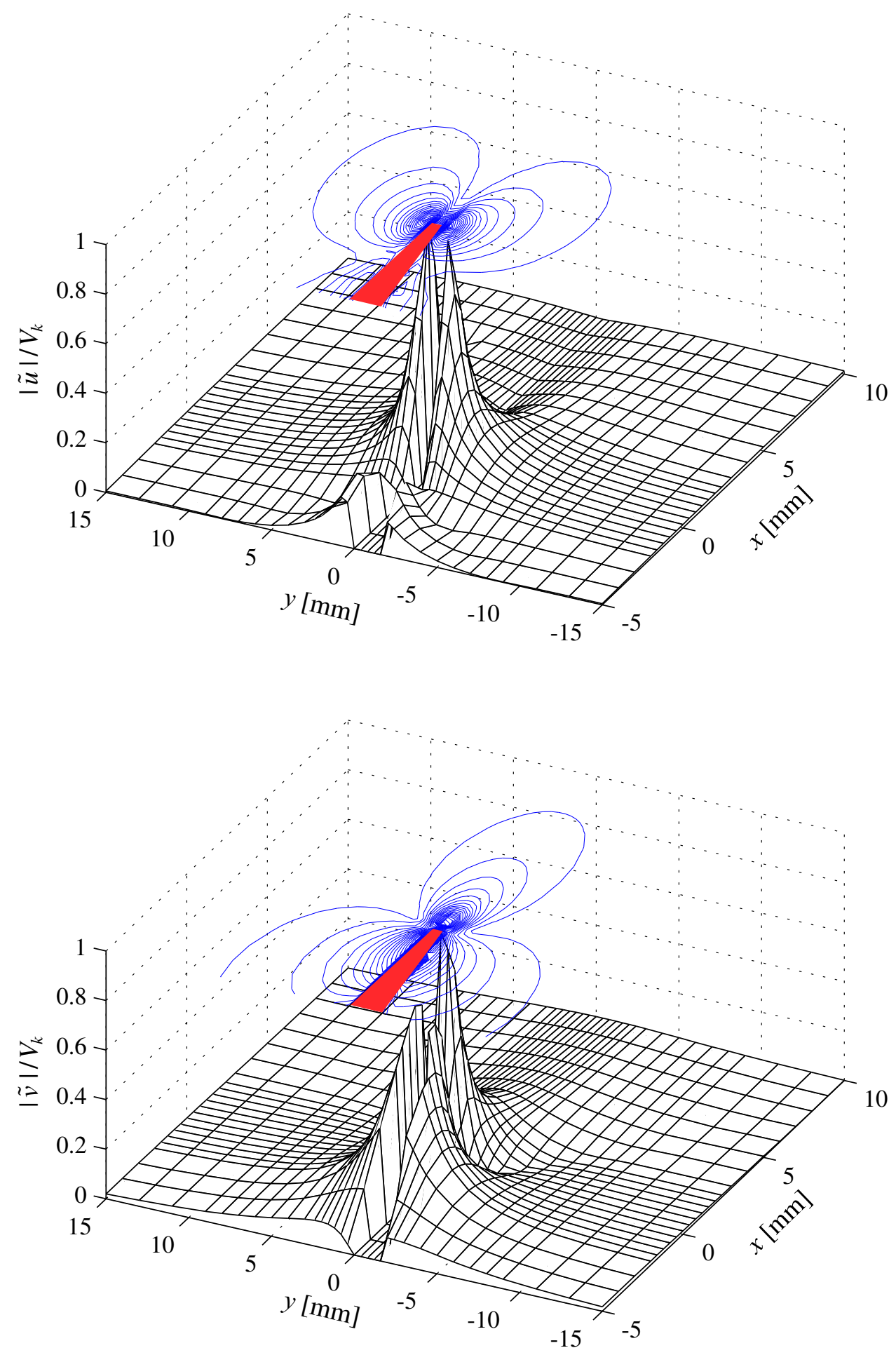

Abb. A.6: Amplitude der Wechselgeschwindigkeit im Bereich der Plattenhinterkante bei Schwingkanten-Anregung mit $2 \mathrm{~Hz}$ : $\tilde{u}$-Komponente (oben) und $\tilde{v}$-Komponente (unten) 


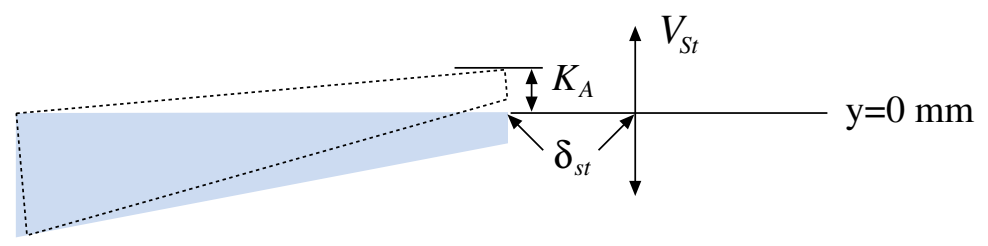

Abb. A.7: Skizze zur Definition von $V_{S t}$ und $K_{A}$

\section{A.2.1 Vergleich mit Messwerten an verschiedenen Stellen}

Die Abbildungen A.9 und A.10 zeigen die numerischen Ergebnisse im Vergleich mit experimentellen Daten bei Druckgradienten-Anregung sowie bei SchwingkantenAnregung mit $2 \mathrm{~Hz}$. Der Vergleich zwischen den beiden Ergebnissen ist dabei an verschiedenen Schnitten durchgeführt, die in Abbildung A.8 schematisch dargestellt sind. Diese Schnittlinien schneiden sich an den Punkten $(x, y)= \pm 4 \delta_{S t}, \pm 4 \delta_{S t}$, wobei $\delta_{S t} \approx 0.4 \mathrm{~mm}$ ist. Man kann dabei die Gültigkeit der numerischen Lösung nicht nur im Potenzialbereich beurteilen, sondern auch im Bereich, in dem die Zähigkeit eine Rolle spielt. In jedem Bild sind sowohl die $\tilde{u}$ - als auch die $\tilde{v}$-Komponente dargestellt. Die aufgetragenen Phasen sind dabei auf die Phase der Geschwindigkeit der Membranen für die Druckgradienten-Anregung und der Geschwindigkeit der Hinterkante für die Schwingkanten-Anregung bezogen.

Man sieht, dass die numerische Lösung und die gemessenen Werte in allen Fällen sehr gut übereinstimmen. Die gute Übereinstimmung lässt sich dahingehend deuten, dass das anregende Strömungsfeld bei ruhendem Medium als eine Kombination von Quellen und Senken angesehen werden kann. Eine Abweichung zwischen den Messwerten und der numerischen Lösung erkennt man allerdings in der Nähe der Splitterplatte (siehe Abbildung A.9 (A), (D) und Abbildung A.10 (B) sowie (C)). Diese Abweichung könnte hauptsächlich dadurch verursacht sein, dass das Koordinatensystem bei der Messung nicht vollständig mit dem der numerischen Rechnung übereinstimmt. Wie in Kapitel 2 erläutert, beträgt die Justiergenauigkeit des Messvolumens relativ zur Hinterkante etwa $\pm 0.05 \mathrm{~mm}$. Da in der Stokesschicht der Gradient der Geschwindigkeit groß ist, trägt eine kleine Verschiebung des Koordinatensystems in der Nähe der Splitterplatte zur Abweichung zwischen den numerischen Werten und Messwerten bei.

Interessant ist der Unterschied der Geschwindigkeitsfelder zwischen der Druckgradienten- und der Schwingkanten-Anregung, insbesondere wenn man Schnitte (C) und (D) miteinander vergleicht. Man erkennt, dass die Schwingkanten-Anregung eine lokale Störung darstellt und der größte Teil des Wechselgeschwindigkeitsfeldes sich auf den Bereich stromauf der Splitterplattenhinterkante konzentriert. Der Unterschied zwischen den Phasenverläufen von $\tilde{u}$ und $\tilde{v}$ ist bei der Schwingkanten-Anregung auffällig. Die $\tilde{v}$-Komponente wechselt ihre Richtung etwa an der Stelle, wo die Splitterplatte endet, während sich die Richtung der $\tilde{u}$-Komponente im Bereich stromauf der Hinterkante umkehrt. 


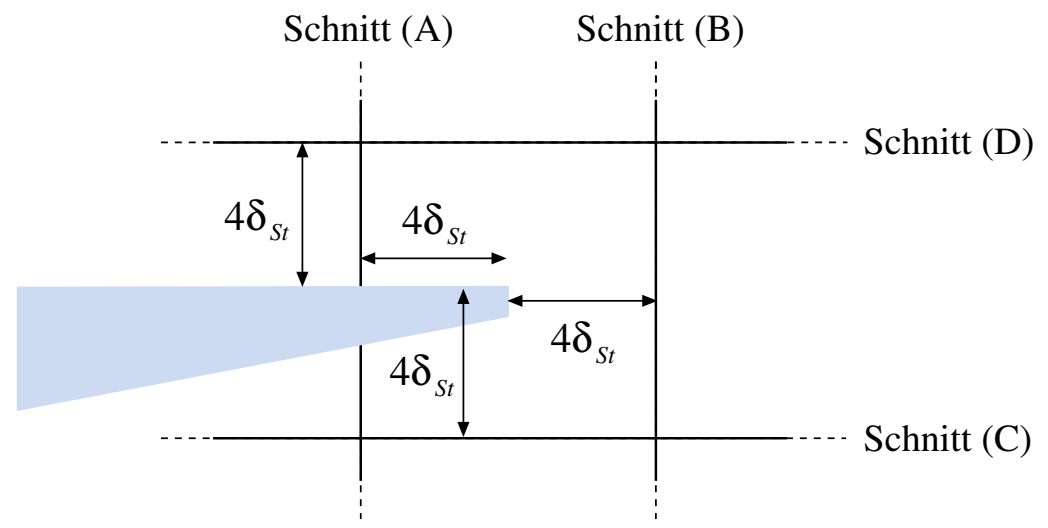

Abb. A.8: Die schematische Darstellung der vier verschiedenen Schnitte zum Vergleich der numerischen Lösung mit dem Experiment

\section{A.2.2 Vergleich mit Messwerten bei verschiedenen Frequen- zen}

Die zu den beiden Anregungsmechanismen gehörigen Potenzialfelder sind frequenzunabhängig. Man erhält daher nahezu identische Kurven, wenn man die Messwerte mit $V_{S t}$ bzw. $V_{k}$ normiert. Aufgrund der Zähigkeit entsteht aber eine Abhängigkeit des Wechselfeldes von der Anregungsfrequenz insbesondere in der Nähe der Splitterplatte. Es ist also zu untersuchen, ob die numerische Simulation die reale Strömung in diesem Bereich richtig beschreibt, denn die durch Überlagerung einer Gleichströmung verursachten Änderungen des Wechselfeldes sollen möglichst genau erfasst werden.

Die Abbildungen A.11 und A.12 zeigen die $\tilde{v}$-Komponente als Funktion von $x$ im Bereich stromab der Plattenhinterkante bei Druckgradienten- bzw. SchwingkantenAnregung und zwar bei vier verschiedenen Frequenzen $\left(0.8 \mathrm{~Hz}\left(\delta_{S t} \approx 0.63 \mathrm{~mm}\right), 1.2 \mathrm{~Hz}\right.$ $\left(\delta_{S t} \approx 0.52 \mathrm{~mm}\right), 1.6 \mathrm{~Hz}\left(\delta_{S t} \approx 0.45 \mathrm{~mm}\right)$ und $\left.2.0 \mathrm{~Hz}\left(\delta_{S t} \approx 0.40 \mathrm{~mm}\right)\right)$. Die geschlossenen Symbole stellen die gemessenen Werte dar. Ebenfalls aufgetragen sind die numerischen Ergebnissen der $\tilde{v}$-Komponente (farbige Linien).

Da die durch den Druckgradienten bedingte Umströmung der Plattenhinterkante, wie in den Arbeiten von Bechert [3], Dierken [8] und Graf [13] berichtet, durch eine Potenzialströmung (die mittels einer konformen Abbildung leicht berechnet werden kann) beschreiben lässt, ist diese Potenziallösung hier zum Vergleich durch die schwarze Kurve dargestellt. Die Geschwindigkeit bei der Potenziallösung der DruckgradientenAnregung $\tilde{v}_{\text {Pot }}^{D r}$ ist dabei $\sim 1 / \sqrt{r}$, wobei $r=\sqrt{x^{2}+y^{2}}$, und der Vorfaktor wurde durch Anpassung am Punkt $x=3 \mathrm{~mm}$ bei einer Anregungsfrequenz von $1.6 \mathrm{~Hz}$ bestimmt wurde.

Die in Abbildung A.11 (a) dargestellte Amplitude von $\tilde{v}$ lässt sich anhand des Vergleichs mit der Potenziallösung in drei Bereiche unterteilen: in einen Stokesbereich, einen Potenzialbereich und einen Übergangsbereich zwischen der Stokes- und der Potenzialströmung. Man erkennt, dass sowohl die gemessenen- als auch die numerischen Werte für große $x$ unabhängig von der Anregungsfrequenz mit der gleichen Steigung wie die Potenziallösung abfallen. Für kleine $x$ weicht die Potenziallösung deutlich von 

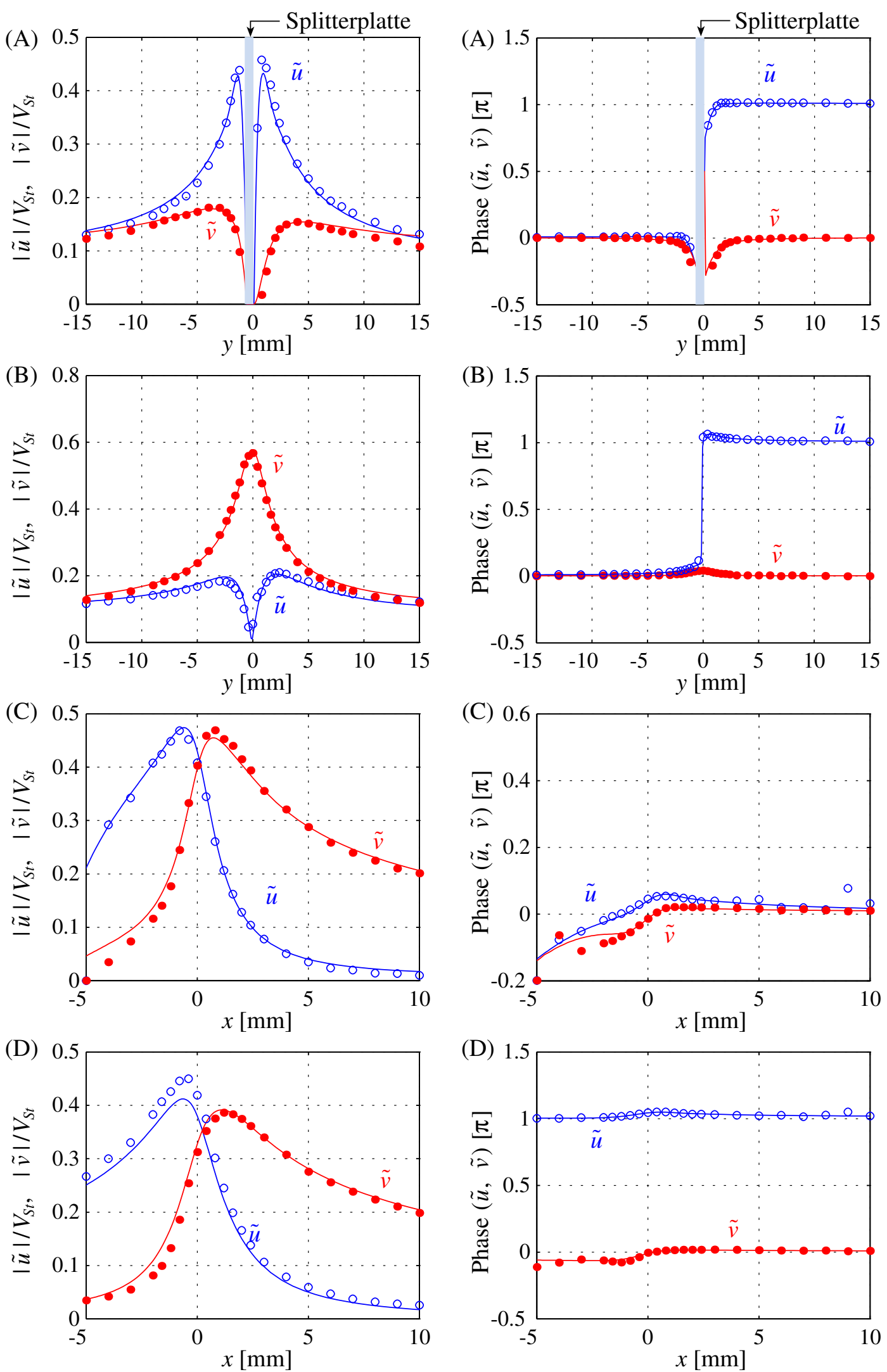

Abb. A.9: Die numerischen Ergebnisse im Vergleich mit experimentellen Werten mit Druckgradienten-Anregung bei $2 \mathrm{~Hz}$ für vier verschiedene Schnitte (A), (B), (C) und (D) (siehe Abbildung A.8); Amplitude (links) und Phase (rechts), Punkte: Messung, Linien: Numerik 

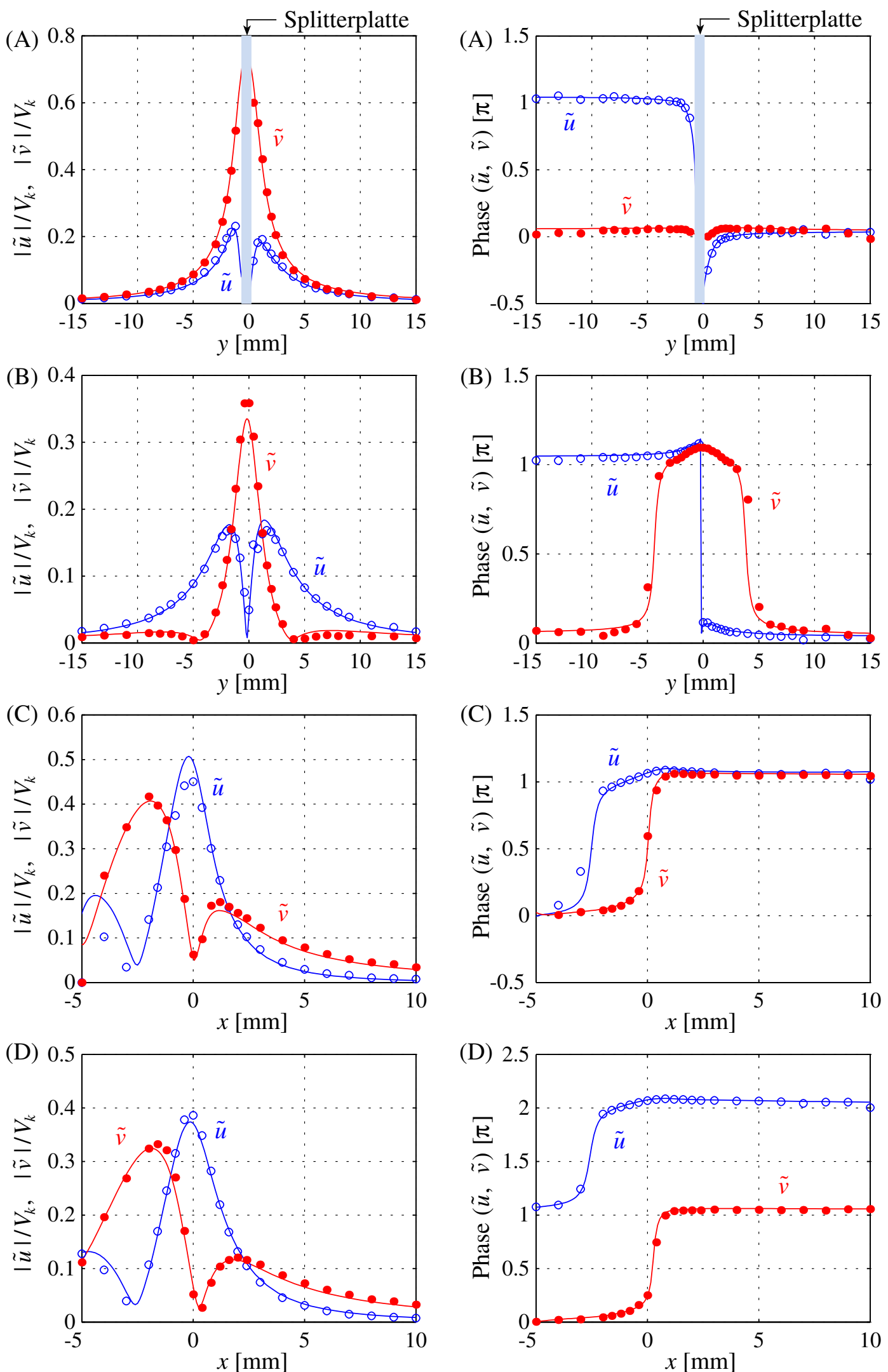

Abb. A.10: Die numerischen Ergebnisse im Vergleich mit experimentellen Werten mit Schwingkanten-Anregung bei $2 \mathrm{~Hz}$ für vier verschiedene Schnitte (A), (B), (C) und (D) (siehe Abbildung A.8); Amplitude (links) und Phase (rechts), Punkte: Messung, Linien: Numerik 
der Messung bzw. dem numerischen Ergebnis ab, was im Bereich der Stokesschicht ja zu erwarten ist.

In der Phase von $\tilde{v}$ als Funktion von $x$ ist der Stokesbereich ebenfalls deutlich zu erkennen (siehe Abbildung A.11 (b)). Die Phase ist dabei auf die Geschwindigkeit der Membranen bezogen. Die Steigung der Phase für kleine $x$ hat eine deutliche Abhängigkeit von der Anregungsfrequenz aufgrund der Dynamik der Stokesschicht, und sie zeigt eine kleine Überhöhung, die durch die Überlagerung von Stokes- und Potenziallösung bedingt ist. In diesem Bereich geht die Stokesschicht in den Potenzialbereich über. Die Potenziallösung ist dabei durch eine konstante Phase gekennzeichnet. Die Phase der gemessenen sowie der numerischen Werte ist im Potenzialbereich nahezu unabhängig von der Frequenz, aber für $x \geq 10 \mathrm{~mm}$ um etwa $0.02 \pi$ gegenüber der Phase der Potenziallösung verschoben.

Der Mechanismus der Schwingkanten-Anregung ähnelt sehr dem der ZylinderAnregung, die von DiERKEn [8] untersucht wurde. Die Stokesschicht am zeitlich harmonisch rotierenden Zylinder wird an der Splitterplatte abgeschält und bewirkt dadurch eine Ausgleichsströmung, die den Charakter einer Potenzialströmung hat. Die Rückströmung bei der Schwingkanten-Anregung entsteht allerdings durch die Schwingung der Hinterkante selbst, da die Kontinuitätsgleichung erfüllt sein muss. Es gibt dabei auch einen Stokesbereich, wo sich die Teilchen mit der Hinterkante mitbewegen. Die Phase der Rückströmung muss daher um $\pi$ gegenüber der Phase der schwingenden Hinterkante gedreht sein.

Der Vergleich zwischen Messung und numerischer Lösung im Fall der SchwingkantenAnregung wird in Abbildung A.12 gezeigt. Bei der Amplitude sind dabei sowohl $\tilde{v}_{\text {Pot }}^{D r}$ als auch die Potenziallösung der Ausgleichsströmung bei der Zylinder-Anregung $\tilde{v}_{P o t}^{Z y}$ zusätzlich aufgetragen. Diese Potenzialströmung, die durch eine oszillierende Dipolquelle am Ende der Splitterplatte angenähert werden kann, ist nun (wie man mit Hilfe der konformen Abbildung berechnen kann) proportional zu $\left(x^{2}+y^{2}\right)^{-3 / 2}$. Beide Potenziallösungen sind an eine Messung mit der Frequenz $1.6 \mathrm{~Hz}$ bei $x=3 \mathrm{~mm}$ angepasst. Es ist zu erkennen, dass die Messwerte für große $x$ ein bisschen flacher ist als $\tilde{v}_{P o t}^{Z y}$, aber deutlich steiler als $\tilde{v}_{P o t}^{D r}$ verlaufen. Da die Rückströmung der Schwingkanten-Anregung eine extrem an der Plattenhinterkante konzentrierte Potenzialströmung darstellt, soll die Schwingkanten-Anregung in Abgrenzung zur Druckgradienten-Anregung als lokale Anregung bezeichnet werden. Im Stokesbereich bzw. im Übergangsbereich zwischen Stokesströmung und Potenzialströmung sieht man deutlich die Abhängigkeit der Amplitude von der Anregungsfrequenz. Dort stimmen die Messwerte mit den numerischen Kurven sehr gut überein. Die Phase der Potenziallösung bei der SchwingkantenAnregung beträgt allerdings $\pi$, während die Phase der Potenzialströmung bei der Druckgradienten-Anregung 0 ist. Die Phase steigt im Stokesbereich mit steigendem Abstand von der Hinterkante um etwa 1.08 $\pi$ an. Dagegen wird bei der DruckgradientenAnregung die Stokesströmung durch die Potenzialströmung verursacht. Die Phase steigt im Stokesbereich mit steigendem Abstand von der Hinterkante auf etwa $0.25 \pi$ an. 

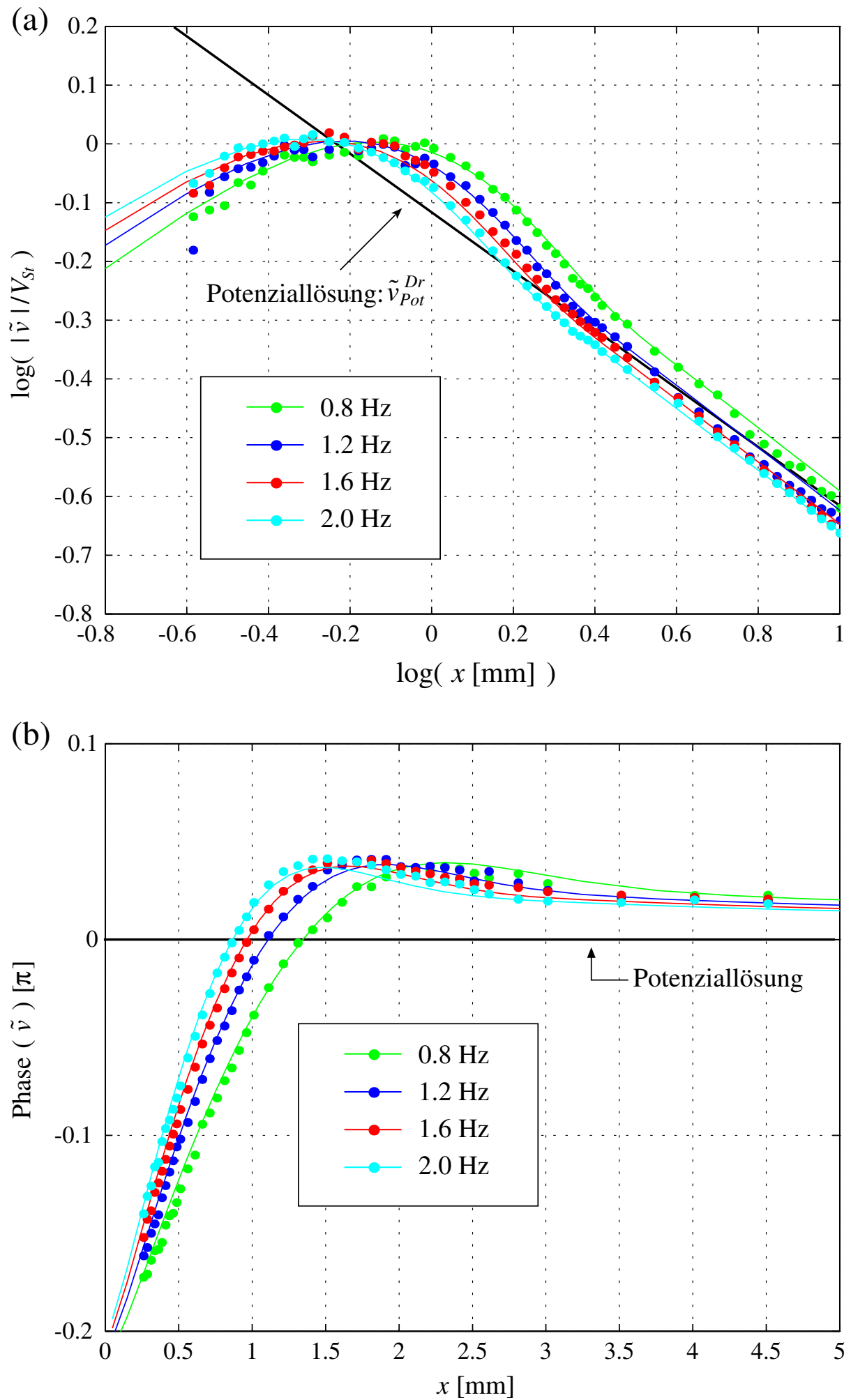

Abb. A.11: Betrag (a) und Phase (b) der $\tilde{v}$-Komponente durch die DruckgradientenAnregung als Funktion von $x$ für vier verschiedene Anregungsfrequenzen bei $y=0 \mathrm{~mm}$ 

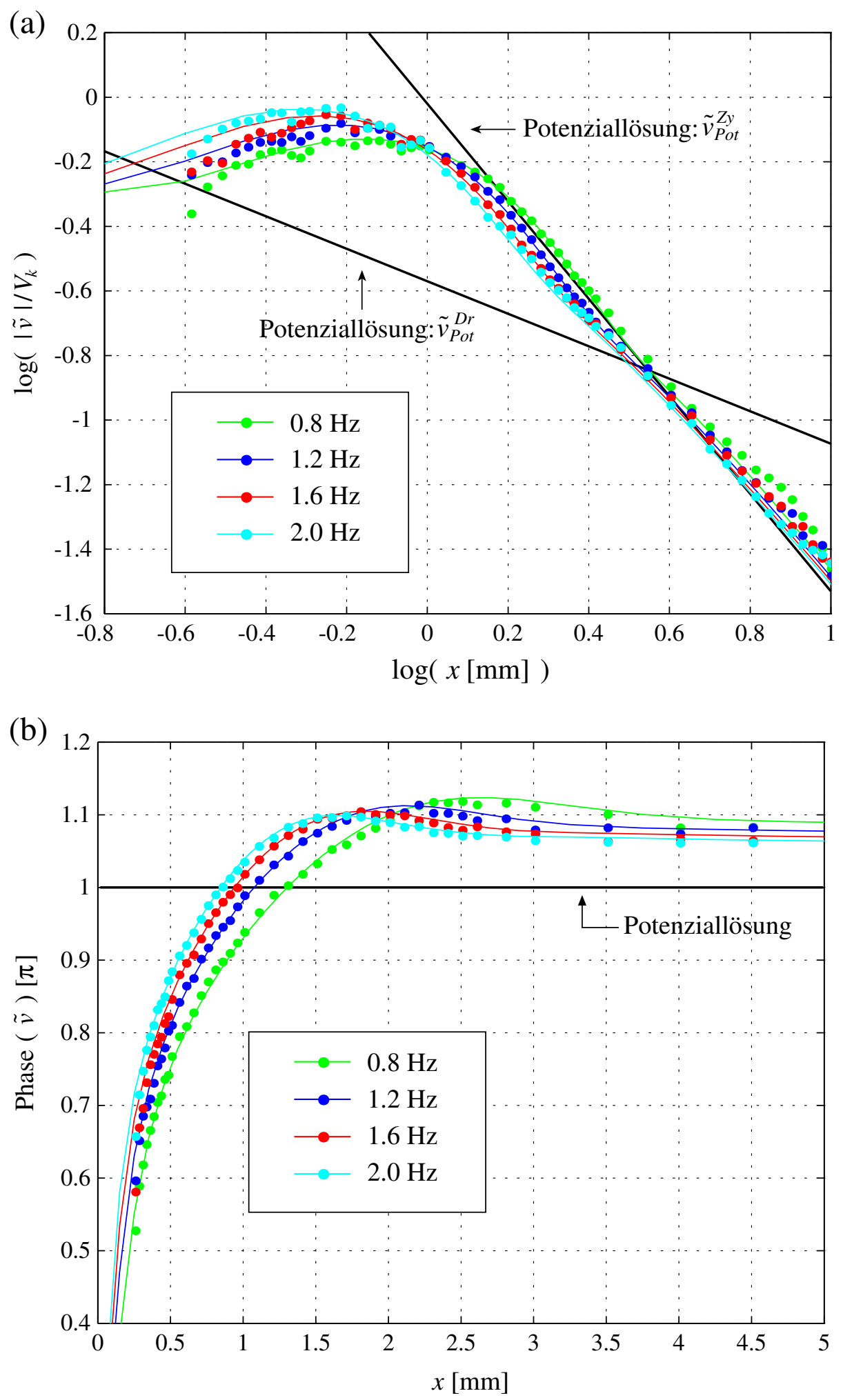

Abb. A.12: Betrag (a) und Phase (b) der $\tilde{v}$-Komponente durch die SchwingkantenAnregung als Funktion von $x$ für vier verschiedene Anregungsfrequenzen bei $y=0 \mathrm{~mm}$ 



\section{Literaturverzeichnis}

[1] Bebber, G. v., 2000, Numerische Untersuchung zur instationären KuttaBedingung, Doktorarbeit, Math.-Nat. Fak. d. Georg-August-Universität zu Göttingen.

[2] Bechert, D., Michel, U., 1975, The control of a Thin Free Shear Layer with and without a Semi-Infinite Plate by a Pulsationg Flow Field, Acustica, Vol. 33, No.5, S.287-307.

[3] Bechert, D., 1982, Excited wave in shear layers, DFVLR-FB 82-23.

[4] Bechert, D., 1988, Excitation of instability waves in free shear layers. Part 1. Theory, J.Fluid Mech., Vol. 186, S. 47-62.

[5] Bechert, D., B. Stahl, 1988, Excitation of instability waves in free shear layers. Part 2. Experiments, J.Fluid Mech., Vol. 186, S. 63-84.

[6] Crighton, D. G., 1985, The Kutta condition in unsteady flow, Ann. Rev. Fluid mech. 186, S. 441-445.

[7] Daniels, P. G., 1985, On the Unsteady Kutta Condition, Q. Jl. Mech. appl. Math., 31, Pt. 1, S. 49-75

[8] Dierken, C., 1995, Rezeptivität einer ablösenden Strömungsscherschicht bei hohen Frequenzen, Diplomarbeit, III. Physikal. Institut der Universität Göttingen.

[9] Enghardt, L., 1996, Adaptive Unterdrückung der Kelvin-Helmholtz-Instabilität einer zweidimensionalen laminaren Scherschicht, Doktorarbeit, Math.-Nat. Fak. d. Georg-August-Universität zu Göttingen.

[10] Freymuth, P., 1966, On transition in a separated laminar boundary layer, J.Fluid Mech., Vol. 25, S. 683-704.

[11] Gaster, M., Kit, E., Wygnanski, I., 1985, Large-scale structures in a forced turbulent mixing layer, J.Fluid Mech., Vol. 50, S. 23-39.

[12] Godreche, C., Manneville, P., 1998, Hydrodynamics and Nonlinear Instabilities, Cambridge University Press. 
[13] Graf, R., 1998, Instationäre Strömungsablösung und Wirbeltransport an der Hinterkante einer Splitterplatte, Diplomarbeit, III. Physikal. Institut der Universität Göttingen.

[14] Grützmacher, V., 1993, Unterdrückung von Instabilitätswellen in einer dreidimensionalen abgelösten Scherschicht, Doktorarbeit, Math.-Nat. Fak. d. GeorgAugust-Universität zu Göttingen.

[15] Michalke, A., 1965, On spatially growing disturbances in an inviscid shear layer, J.Fluid Mech., Vol. 23, S. 512-544.

[16] Möhring, W., 1975, On flows with vortex sheets and solid plates, J.Sound Vibr. Vol. 38(3), S. 403-412.

[17] Neuwald, P., 1970, Anregung der Kelvin-Helmholtz-Instabilität durch eine einlaufende Tollmien-Schlichting-Welle, Doktorarbeit, Math.-Nat. Fak. d. GeorgAugust-Universität zu Göttingen.

[18] Orszag, S. A., Crow, S. C., 1970, Instability of a Vortex Sheet Leaving a Semi Infinite Plate, Stud. in Appl. Math., Vol. XLIX No.2, S. 167-181.

[19] Poling, D. R., Telionis, D. P., 1986, The Response of Airfoils to Periodic Disturbances - The Unsteady Kutta Condition, AIAA Journal, 24(2), S. 193-199

[20] Rebel, J., 1992, Aktive Beeinflussung einer ablösenden dreidimensionalen Plattengrenzschicht mittels Tollmien-Schlichting-Wellen, Doktorarbeit, Math.-Nat. Fak. d. Georg-August-Universität zu Göttingen.

[21] Satyanarayana, B., Davis, S., 1978, Experimental studies of unsteady trailingedge conditions, AIAA Journal, 16(2), S. 125-129

[22] Schaaf, K., 1988, Experimentelle Untersuchung zur Gültigkeit der instationären Kutta-Bedingung bei hohen Strouhalzahlen, Doktorarbeit, Math.-Nat. Fak. d. Georg-August-Universität zu Göttingen.

[23] Schmitz, S., 2004, Instationäre Strömungsablösung an der Hinterkante einer Splitterplatte, Diplomarbeit, III. Physikal. Institut der Universität Göttingen.

[24] Schlichting, H., 1982, Grenzschicht-Theorie, Verlag G. Braun, Karlsruhe, 8. Auflage.

[25] Steinhoff, W., Ebrecht, W., Geiling, T., 1994, PC-programmierbare Schrittmotorsteuerung, interner Bericht, III. Physikal. Institut der Universität Göttingen.

[26] Yabe, T., Aoki, T., 1991, A universal solver for hyperbolic equations by cubicpolynomial interpolation I; One dimensional solver, Computer Physics Comunications, Vol. 66, S. 219-232.

[27] Yabe, T., Uchiumu, T., Ogata, Y., 2003, CIP-Methode, Morikitashuppan. 


\section{Danksagung}

Diese Arbeit habe ich am Dritten Physikalischen Institut der Universität Göttingen unter der Anleitung von Herrn Prof. Dr. Dirk Ronneberger angefertigt. Bei ihm möchte ich mich für die sehr gute Betreuung und die freundliche Atmosphäre herzlich bedanken. Sein Interesse an meiner Arbeit, die Bereitschaft, mich jederzeit bei der Lösung aller auftretenden Probleme und Fragen zu unterstützen, und die daraus entstehenden vielen interessanten Diskussionen haben mich sehr bereichert.

Bei Herrn Prof. Dr. Werner Lauterborn bedanke ich mich für die Übernahme des Korreferates und für die sehr nette Arbeitsumgebung.

Herrn Prof. Dr. Helmut Eckelmann danke ich für seine freundliche und hilfsbereite Unterstützung während meiner ganzen Promotionszeit.

Ohne die tatkräftige Unterstützung durch die feinmechanische und die elektronische Werkstatt des Instituts wäre die Durchführung der Experimente nicht möglich gewesen. Bei Herrn Dieter Hille und bei Herrn Thomas Jonigkeit bedanke ich mich für die gute Zusammenarbeit beim Aufbau der Schwingkanten-Anregung. Herrn Dr. Karl Lautscham danke ich für die Unterstützung beim Umbau des Lasers. Ich danke Herrn Thomas Geiling dafür, dass er mir häufig Ideen für die Herstellung der angenehmen Rechnerumgebung gegeben hat.

Bei Herrn Dr. Dieter Guicking bedanke ich mich dafür, dass er mir Herrn Prof. Dr. Dirk Ronneberger vorgestellt hat.

Bei dem Mitgliedern der Arbeitsgruppe „Strömungsakustik“, die während meiner Promotion zu der angenehmen Arbeitsatmosphäre beigetragen haben, bedanke ich mich für ihre Unterstützung. Besonders erwähnen möchte ich dabei Matthias Jüschke, der nicht nur das Korrekturlesen übernommen hat, sondern auch auf jede Diskussion über meine Arbeit eingegangen ist.

Ebenfalls danke ich allen Mitgliedern des Dritten Physikalischen Instituts, die mir im Laufe meiner Arbeit auf die eine oder andere Weise geholfen haben. 
Ich möchte mich an dieser Stelle auch bei der Familie Großer, besonders Monika und Lucian herzlich bedanken, ohne deren liebevoller Unterstützung hätte ich mehr Schwierigkeiten gehabt in Deutschland zu leben.

Mein besonderer und herzlicher Dank aber gilt meiner Freundin Marie-Louise, die nicht nur die Mühsal des Korrekturlesens übernommen hat, sondern mich auch immer wieder daran erinnert hat, dass es neben der Physik auch noch andere schöne Dinge im Leben gibt. 


\section{Lebenslauf}

Am 23. Dezember 1973 wurde ich als Sohn der ehemaligen Lehrerin Mie Tokuno geb. Kawamura und des Reisebüromanagers Mitsutaka Tokuno in Sakata-Yamagata/Japan geboren. Im Anschluss an die sechsjährige Grundschulzeit vom Frühling 1980 bis 1986 besuchte ich die Mittelschule Wanagaya in Chiba vom Frühling 1986 bis 1989. Danach besuchte ich die Oberschule Hongo in Tokyo. Dort legte ich im März 1992 das japanische Abitur "Kotogakko Sotsugyo“ ab. Im Sommersemester 1992 nahm ich das Ingenieursstudium an der Tokyo Denki Unversität in Tokyo auf. Nach acht Semestern bestand ich im März 1996 die Prüfung für „Bachelor of Engineering“. Im April 1996 begann ich am Akustischen Institut unter der Anleitung von Prof. Dr. Hareo Hamada mit der Anfertigung meiner Magisterarbeit zum Thema „Erzeugung dreidimensionaler Schallereignisse mit Digitalsignalverarbeitung“. Die Magisterprüfung legte ich im März 1998 ab. Im Mai 2000 begann ich unter der Anleitung von Prof. Dr. Dirk Ronneberger mit den Arbeiten zur Promotion. Vom Mai 2000 bis April 2003 erhielt ich ein Stipendium im Rahmen des Graduiertenkollegs „Strömungsinstabilitäten und Turbulenz“. Seit September 2003 bin ich als wissenschaftlicher Mitarbeiter am Dritten Physikalischen Institut tätig. 